\title{
Balancing between Energy, Comfort and MONEY \\ In Control of Residential Electric Tank Water Heaters
}


Members of the dissertation dissertation committee:

Chairman and secretary:

Promoter:

Prof. dr. ir. G.J.M. Smit

Prof. dr. J.L. Hurink

Prof. dr. ir. A.K. Hajdasinski

Prof. dr. ir. M. Aiello
Prof. dr. J.N. Kok

Prof. dr. P.J.M. Havinga

University of Twente

University of Twente

Neyednrode Business Universiteit

Universität Stuttgart
University of Twente

University of Twente
Rijksdienst voor Ondernemend

Nederland
Parts of this research have been carried out within GoGreen project supported by Rijksdienst voor Ondernemend Nederland.

Printed by: $\quad$ IPSKAMP Printing

Cover art: https://unsplash.com/photos/U3C79SeHa7k

Cover design: $\quad$ IPSKAMP Printing

ISBN 978-90-365-5067-3

DOI 10.3990/1.9789036550673

Copyright $\odot 2020$ by Alexander Belov

All rights reserved. No part of this book may be reproduced or transmitted, in any form or by any means, electronic or mechanical, including photocopying, microfilming, and recording, or by any information storage or retrieval system, without the prior written permission of the author. 


\section{Balancing between Energy, Comfort and MONEY \\ In Control of Residential Electric Tank Water Heaters}

\section{PROEFSCHRIFT}

ter verkrijging van de graad van doctor aan de Universiteit Twente,

op gezag van de Rector Magnificus prof. dr. T.T.M. Palstra, volgens besluit van het College voor Promoties,

in het openbaar te verdedigen

op woensdag 7 oktober 2020 om 12.45 uur

door

\section{Alexander BELOV}

geboren op 4 april 1987 te Ufa, Rusland. 
Dit proefschrift is goedgekeurd door de promotor:

Prof.dr. P.J.M. Havinga 
Per aspera ad astra 



\section{ACKNOWLEDGMENTS}

It has been a long and thorny way to accomplish this work with the quality that I am proud to present to a curious reader. However, I was not alone on this way and I would like to take the liberty to express here my sincere gratitude to all those who were there with me.

I would like to thank my promoter and supervisor Paul Havinga for a nice opportunity to do a scientific research in the Netherlands. I remember how during our first interview he and my former supervisor Nirvana Meratnia were asking me many questions about my scientific interests and how I myself wanted to conduct the future research. After the interview, all my thoughts were already in the world of energy savings and residential consumers. Shortly, I found myself in a nice and very multicultural environment of Pervasive Systems research group at the University of Twente. My sincere thanks to both Paul and Nirvana for inspiring my research during the years. Not always the things were going according to the plan, which was mainly due to a novelty of the research topic to us, nonetheless, we successfully managed to find the light at the end of the tunnel and to go through.

My special thanks are dedicated to Gerard Smit whose recommendations and interesting scenarios significantly enriched my work. I am taking my hat off to fohann Hurink for the time he spent helping me refine this thesis. His willingness to understand the problems that we solved and his always original view on them helped me see the work in a different light and tremendously improve it. Many thanks to both of them for their help.

I would like to express my thanks to all my former colleagues for a great time that we had together in Zilverling UT. Thank you guys for your inspiring weekly pitches in Rappa, movie nights, cultural borrels, water skiing, baking pasta and cooking traditional food together, interesting talks at canteen and thought-provoking coffee breaks. My close friendship with Jan Peter Mayers, Viktorio El Hakim and Saeid Yazdani made my time at UT really precious. Many thanks and appreciation to my friends Wouter van Kleunnen, Bram Dil, Muhammad Shoaib, Fatjon Seraj, Viet Duc Lee, Vignesh R.K. Ramachandran, Mitra Baratchi and her husband Siavash Aflaki.

A working atmosphere during the weekly meetings in the Energy group (CAES) encouraged me a lot. There, I met enthusiastic people keen on research in various energy related fields. Thanks to Firka Fink for his useful remarks and to Richard van Leeuwen for his interest and willingness to help.

I would like to show appreciation to my co-authors Berend fan Van der Zwaag, Aleksandr Vasenev and Vadim Kartak for their valuable input, justified remarks and just for a nice time discussing our following publications. I would especially like to express my deepest gratitude to Vadim Kartak with whom we worked on a discretized approach to the problem and who helped me with different mathematical questions. I would also like to thank Haidar Gumerov for enlightening me with thermodynamics aspects related to the research.

Thanks to Nicole Baveld, Marlous Weghorst and Thelma Nordholt for their devotion and enthusiasm at work. They made lives of many $\mathrm{PhDs}$ a bit brighter. 
I would finally like to thank two people without who this work would have never been finished. My deepest gratitude and warm hugs are for my girlfriend Anna M.L. Machiels for encouraging me to finish the thesis during these years. Thanks for your valuable input about hypothalamus (even though I had to remove it). Special thanks to my mom Inna for cheering me up and motivating me. 


\section{SUMMARY}

With an advent of smart meters backed up by IT infrastructure and smart grid, residential electricity consumers are getting more involved into the energy market and in the management of their own demand today. Transition of the demand management in electrical grids towards the load management implemented on the consumer's side stimulates a shift of the role of residential electricity consumers too.

Along with new technological advances such as renewables and energy storages, there is a need to manage these technologies efficiently. Although much progress the last years was done in direction of management of electricity demand on the consumer's side, Demand Response (DR) programs have not yet become very popular.

An example of DR is adapting the start and end times of household appliances in response to variable electricity prices or in order to avoid a peak demand in the grid. However, such an adapted operation of domestic appliances may not always be convenient for residents. Despite possible energy and money savings, new operation can heavily interfere with occupants' lifestyles so that they might refrain to participate in DR.

The motivation of our research is therefore to increase the acceptance of efficient DR systems by involving consumers in the management of their own electricity consumption. Our assumption is that once a regular consumer can consciously decide how much comfort a modified use of energy can cost him, and on the other hand how much savings it can bring him, he/she will take more responsibility in managing personal energy needs.

The aim of the study is to explore how residential consumers can participate in DR with minimal loss of personal comfort.

In this thesis, we investigate how consumer goals such as profit maximization from DR and minimization of end-user comfort conflict with each other, and how they can be unified in DR, looking specifically at an exemplary case of residential electric tank water heaters (WHs). In essence, this addresses the problem of balancing (sometimes) conflicting goals of minimizing energy consumption, maximizing money savings, and minimizing user discomfort during hot water activities.

This thesis deals with a possible solution for WHs that allows a household to maximize energy and money savings for water heating while highly respecting end-user comfort. The proposed mechanism thus balances between the following objectives of load management: minimizing electricity consumption, minimizing heating costs and minimizing disruptions of user comfort. By finding trade-off(s) between the desired user comfort and the desired level of energy (money) savings, the model creates an appropriate water heating program for the WH for the coming day.

Based on simulations of the proposed control mechanism, we can conclude that our solution is capable of balancing conflicting consumer goals. Although the mechanism has been simulated on water heating systems (boiler), the method can also be applied to other equipment in households. 



\section{SAMENVATTING}

Door de komst van slimme meters die verbonden zijn met een IT-infrastructuur en smart grid, worden bewoners van huizen steeds meer betrokken bij de energiemarkt en bij het beheer van hun eigen vraag naar energie. De overgang van vraagbeheer in elektriciteitsnetten naar het belastingsbeheer dat aan de kant van de consument wordt geïmplementeerd, zorgt daardoor voor een verschuiving van de rol van huishoudelijke elektriciteitsverbruikers.

Naast het gebruik van nieuwe technologische ontwikkelingen zoals hernieuwbare energiebronnen en energieopslag, is het ook nodig om deze technologieën efficiënt te gebruiken. Hoewel er de afgelopen jaren veel vooruitgang is geboekt in de richting van het beheer van de elektriciteitsvraag aan de kant van de consument, zijn Demand Response (DR) programma's echter nog niet erg populair geworden.

Een voorbeeld van een DR systeem is het aanpassen van de start en eindtijden van huishoudelijke apparatuur aan de prijs van de elektriciteit, waarbij ook een piekvraag in het net kan worden vermeden. Een dergelijke aangepaste werking van huishoudelijke apparatuur is echter niet altijd prettig of handig voor bewoners omdat het de levensstijl van bewoners ernstig kan verstoren. Ondanks de mogelijke energie- en geldbesparingen die het voor de bewoners teweeg kan brengen, wordt het negatief effect op comfort vaak als te groot ervaren.

De motivatie van ons onderzoek is daarom om de acceptatie van efficiente DR systemen te vergroten door de consument bij het beheer van hun eigen elektriciteitsverbruik te betrekken. Onze aanname is dat zodra een gewone consument bewust kan beslissen hoeveel comfort een gewijzigd gebruik van energie hem kan kosten, en het hem aan de andere kant aan besparingen kan opleveren, hij/zij meer verantwoordelijk zal nemen bij het beheren van zijn energiebehoeften.

Het doel van het onderzoek is om te verkennen hoe residentiële consumenten kunnen deelnemen aan DR met minimale vermindering van hun persoonlijk comfort.

In dit proefschrift onderzoeken we hoe consumentendoelen zoals winstmaximalisatie van DR en minimalisering van het comfort van de eindgebruiker met elkaar in conflict komen, en hoe ze kunnen worden verenigd in DR, waarbij we specifiek als exemplarisch voorbeeld kijken naar residentiële elektrische tankboilers (WH's).

In essentie behandelen hiermee het probleem van het in evenwicht brengen van (soms) tegenstrijdige doelen van minimalisatie van het energieverbruik, maximalisatie van geldbesparingen en minimalisering van ongemak voor de gebruiker tijdens energie-vragende activiteiten.

We bespreken in dit proefschrift een mogelijke oplossing voor WH's waarmee een huishouden energie- en geldbesparingen voor waterverwarming kan maximaliseren terwijl het comfort van de eindgebruiker in hoge mate wordt gerespecteerd. Het voorgestelde mechanisme balanceert hiermee tussen de doelstellingen van het belastingsbeheer: minimalisatie van het elektriciteitsverbruik, minimalisering van verwarmingskosten en minimalisering van verstoringen van het gebruikerscomfort. Door een afweging te maken tussen het 
gewenste gebruikerscomfort en het gewenste niveau van energie (geld) besparing, creëert het model een passend waterverwarmingsprogramma voor de boiler voor de komende dag.

Op basis van simulaties van het voorgestelde controlemechanisme kunnen we concluderen dat onze oplossing in staat is om een afweging te maken tussen de tegenstrijdige consumentendoelen. Alhoewel het mechanisme is gesimuleerd op waterverwarmingssystemen (boiler), kan de methodiek ook bij andere apparatuur in huishoudens worden toegepast. 


\section{Contents}

Acknowledgments

vii

Summary

ix

Samenvatting $\quad$ xi

1 Introduction $\mathbf{1}$

1.1 Challenges in Modern Electricity Grids. . . . . . . . . . . . . . . . . . . 3

1.2 Residential Water Heating and Electric Tank Water Heaters . . . . . . . . 5

1.2.1 Water Heating in Household Electricity Consumption . . . . . . . 5

1.2.2 Park of Electric Tank Water Heaters . . . . . . . . . . . . . . . . 6

1.2.3 Water Heaters as Flexible Loads . . . . . . . . . . . . . . . . . . 7

1.3 Demand Response of Water Heaters . . . . . . . . . . . . . . . . . . . . 7

1.3.1 Incentive-based Demand Response. . . . . . . . . . . . . . . . . 7

1.3.2 Price-based Demand Response . . . . . . . . . . . . . . . . . . . 8

1.4 Challenges of Demand Response . . . . . . . . . . . . . . . . . . . . 9

1.4.1 Consumer Acceptance of Demand Response . . . . . . . . . . . . 9

1.4.2 Impacts of Demand Response of Water Heaters on User Comfort. . 10

1.4.3 Energy Efficiency of Water Heaters in Demand Response . . . . . 10

1.5 Research Scope and Problem Statement . . . . . . . . . . . . . . . . . . 11

1.5.1 Energy Efficient Control . . . . . . . . . . . . . . . . . . 11

1.5.2 User Comfort Satisfaction . . . . . . . . . . . . . . . . . . . 12

1.5.3 Research Questions . . . . . . . . . . . . . . . . . 12

1.6 Approach and Contribution . . . . . . . . . . . . . . . . . . . . . 12

1.7 Thesis Organization . . . . . . . . . . . . . . . . . . 13

2 Related Work $\quad 15$

2.1 Hot Water System at Home . . . . . . . . . . . . . . . . . . . . . . 16

2.2 Control of Electric Tank Water Heaters . . . . . . . . . . . . . . . . . . 17

2.2.1 Incentive-based Control of Water Heaters . . . . . . . . . . . . . 17

2.2.2 Price-based Control of Water Heaters . . . . . . . . . . . . . . . 22

2.2.3 Summary. . . . . . . . . . . . . . . . . . 28

2.3 Necessity of Considering Heat Losses . . . . . . . . . . . . . . . . . . . 32

2.4 Necessity of Considering User Comfort . . . . . . . . . . . . . . . . . . 32

2.4.1 Thermal Comfort Modeling . . . . . . . . . . . . . . . . 33

2.5 Modeling Water Heater Operation . . . . . . . . . . . . . . . . . . . . . 34

2.5.1 Stratified and Well-mixed Tanks . . . . . . . . . . . . . . . . . . 35

2.5.2 Utilized Water Heater Model . . . . . . . . . . . . . . . . . . . . 37

2.5.3 Heat Losses . . . . . . . . . . . . . . . . . . . . . . . . . 40 
2.6 Parameters of Water Heater Model . . . . . . . . . . . . . . . . . . . . . 40

2.7 Summary. . . . . . . . . . . . . . . . . . . . . . . 42

3 User Comfort Modeling $\quad 45$

3.1 Premises for User Comfort Modeling . . . . . . . . . . . . . . . . . . . . 47

3.1.1 Specifics of Human Comfort during Hot Water Usage . . . . . . . 47

3.1.2 Factors Influencing User Comfort . . . . . . . . . . . . . . . . . 49

3.1.3 Environmental and Personal Variables . . . . . . . . . . . . . . . 49

3.2 Thermal Comfort Modeling . . . . . . . . . . . . . . . . . . . 52

3.3 Flow Comfort Model . . . . . . . . . . . . . . . . . . . . 56

3.4 Conclusion. . . . . . . . . . . . . . . . . . . . . . . . 59

4 Maintaining Comfort for Single Water Activity $\quad 61$

4.1 User Discomfort in Deadband Control . . . . . . . . . . . . . . . . . . . 66

4.2 Organization of Chapter . . . . . . . . . . . . . . . . 66

4.3 Water Pre-heating Strategy. . . . . . . . . . . . . . . . . . . . . . . 67

4.3.1 Pre-heating Problem . . . . . . . . . . . . . . . . . . 67

4.3.2 Pre-heating Problem Clarification . . . . . . . . . . . . . 70

4.3.3 Assumptions . . . . . . . . . . . . . . . . . 70

4.4 Proposed Pre-heating Control . . . . . . . . . . . . . . . . . . . . . . . 72

4.4.1 Problem Constraints . . . . . . . . . . . . . . . . . . . 72

4.4 Objective Function . . . . . . . . . . . . . . . . . . 72

4.4.3 Performance Evaluation . . . . . . . . . . . . . . . . . 74

4.4.4 Results Discussion and Conclusion. . . . . . . . . . . . . . . . 80

4.5 Flow Control Strategy . . . . . . . . . . . . . . . . . . . . . . . . . . . 82

4.5.1 Standard Solutions to Support Stable Tap Water Temperature . . . 83

4.5.2 Flow Control Principle . . . . . . . . . . . . . . . . . . . . . . . 84

4.5.3 Problem of Coupling Flow Control with Water Heating . . . . . . 85

4.6 Proposed Combined Pre-heating and Flow Control. . . . . . . . . . . . . 87

4.6.1 Problem Constraints . . . . . . . . . . . . . . . . . . . . 89

4.6.2 Objective Functions . . . . . . . . . . . . . . . . . . . . . . . . 90

4.6.3 Performance Evaluation . . . . . . . . . . . . . . . . . . 91

4.6.4 Results Discussion and Conclusion. . . . . . . . . . . . . . . . . 94

4.7 Conclusion. . . . . . . . . . . . . . . . . . . 96

5 Control Strategies For Multiple Water Activities 99

5.1 Residential Hot Water Consumption . . . . . . . . . . . . . . . . . . . . 103

5.2 Limitations of Conventional Deadband Control of Water Heaters . . . . . 103

5.3 Organization of Chapter . . . . . . . . . . . . . . . . . 105

5.4 Energy Model . . . . . . . . . . . . . . . . . . . . . . . 105

5.4.1 Preliminary Discussion and Problem Formulation . . . . . . . . . 105

5.4 Our Approach . . . . . . . . . . . . . . . . . . . . 108

5.4.3 Assumptions. . . . . . . . . . . . . . . . . . . . 114

5.4.4 Proposed Energy Model . . . . . . . . . . . . . . . . . . . 115

5.4.5 Proposed Control Mechanism . . . . . . . . . . . . . . . . 116

5.4.6 Intra-day Timescale . . . . . . . . . . . . . . . . . . 118

5.4.7 WA Timescale . . . . . . . . . . . . . . . . . . . . 118 
5.4.8 Formalization of Energy Model. . . . . . . . . . . . . . . . . . . 119

5.4.9 Performance Evaluation . . . . . . . . . . . . . . . . . . . 123

5.5 Price Model . . . . . . . . . . . . . . . . . . . . . . . . . . 146

5.5.1 Preliminary Discussion and Problem Formulation . . . . . . . . . 146

5.5.2 Our Approach . . . . . . . . . . . . . . . . . . . . . . 149

5.5.3 Assumptions . . . . . . . . . . . . . . . . . . . 149

5.5.4 Price Model . . . . . . . . . . . . . . . . . . . . . . . . . . . . 149

5.5.5 Formalization of Price Model. . . . . . . . . . . . . . . . . 150

5.5.6 Performance Evaluation . . . . . . . . . . . . . . . . . 151

5.6 Conclusion. . . . . . . . . . . . . . . . . . . . . 159

6 Conclusion $\quad 161$

$\begin{array}{lr}\text { Bibliography } & 167\end{array}$

$\begin{array}{ll}\text { List of Publications } & 177\end{array}$ 



\section{1 \\ INTRODUCTION}

$\mathrm{D}$ emand-side management (DSM) refers to a broad portfolio of policies and measures to modify consumers' electricity usage patterns. The practice of DSM has a long history. The energy crises in 1970s caused by rising prices, declining supplies of oil and natural gas, and new environmental pressures illuminated inefficiencies of the regulatory structure of the power market to coordinate the levels of power supply and demand [1]. This began the age of the market deregulation characterized by entrance of competing parties in the electricity market. At the same time, in response to the growing concerns about dependence on fossil fuels, the idea of management of the energy demand at the customer side of the electricity meter came into play. Electric utility DSM programs rapidly evolved in late 1980s reached their largest size in the US in 1993 [2]. With deregulation of the European electricity market at the end of the 1990s, the energy community regained its interest in DSM and particularly in demand response.

The European power system undergoes significant changes in generation, transportation, distribution and utilization of electricity these days. This can be characterized by a growing penetration of decentralized and renewable energy sources (RES) that represent an intermittent (weather-dependent) electricity supply, energy storages to utilize energy excesses, new types of consumer loads (e.g., electrical vehicles and products with A+++ energy labels), ICT advances (e.g., wireless controllers and data centers) and consumers' flexibility to reduce their energy consumption which can be seen as commodity to be purchased by energy market parties.

In the context of current changes, the task of maintaining the reliability of the power supply gets under a spotlight. The power systems are facing challenges such as accommodating a highly-variable and less controllable RES, and reducing peak demands. To avoid or defer large investments in grid infrastructures and storage facilities, already existing consumption can be enabled with control capabilities [3]. In that regard, demand response (DR) is commonly recognized as an important instrument for improving the energy efficiency and stability of the European electrical grid [4].

Basically, DR programs and tariffs are tailored to motivate consumers to reduce their electricity usage at times of high market prices or when grid reliability is jeopardized [5]. Together with measures for energy efficiency and energy conservation, DR rests under 
the umbrella of DSM programs. Demand response can give numerous benefits both for participants and market-wide. Key benefits can be summarized as follows [6-9]:

- Market-wide:

- reduction of costs due to optimal utilization of electricity network infrastructure. Utility companies gain the possibility to shape the electricity demand curve and spread it more evenly throughout the day. As a result, the electrical equipment (e.g., turbines, transformers) can work more efficiently which means the reduced maintenance costs and the increased reliability of the power supply. Besides, the electricity price is expected to reduce because of a better coordination of generation reserves (energy can be consumed from cheap generators).

- greater flexibility in the management of the system and increased reliability. Utility companies can manage consumer loads in order to resolve grid congestions and system failures. For example, a group of residential water heaters can be shedded during the peak demand period upon a request of the energy company, resulting in a short-term increase of the generation capacity and stability of supply. In turn, electricity consumers can reap the benefits of the reduced power outages.

- support of the renewable generation, i.e. DR can provide a balancing resource for RES.

- decreased (or deferred) need for local network investments. Since electricity consumption can be shifted away from peak demand hours, investments in new generation capacities and distribution infrastructure in regions with tight network capacity can be avoided (or deferred), which reflects in reduced electricity prices for consumers and a lower need for coal and gas.

- Consumers:

- incentive payments and bill savings. Participants of DR can receive financial rewards from the utility company and(or) can profit from cheaper electricity during off-peak price periods.

- optimized electricity consumption. For example, residential consumers interested in decrease of their environmental footprints can reduce their energy consumption with the help of load scheduling solutions (e.g., home energy management systems).

- access to the electricity market. With the help of DR, consumers with local generation (e.g., solar panels) and energy storages can increase their "selfconsumption" and sell the surpluses of local energy (e.g., feed-in tariffs).

Despite multiple benefits of DR, it is currently not widely acknowledged between consumers. As the energy reports $[8,10]$ reveal, consumers pay attention not only to financial benefits of DR but also to their personal comfort. In particular, residential consumers are afraid of loosing control of their own home appliances and concerned that their comfort might degrade when enrolling their loads in DR. If such a dissatisfaction outweighs the consumer goal to benefit financially from DR, consumers might choose to 
opt out, which will also have an overall negative impact on the trust to the utility company. Therefore, consumer comfort can be seen as a potential enabler of regained consumer trust and acceptance of DR.

The motivation of our research is therefore to increase the acceptance of efficient DR systems by engaging consumers in the management of their own electricity consumption. We assume that once a regular consumer can consciously decide how much comfort a modified use of energy can cost him, and how much savings he can gain in return, he/she will take more responsibility in managing personal energy needs.

The aim of the study is to explore how residential consumers can participate in DR with minimal loss of personal comfort. We investigate how to reconcile possibly conflicting consumer goals such as maximization of profits and minimization of user comfort disruptions, looking specifically at an exemplary case of residential electric tank water heaters (WHs). This thesis deals with a possible solution for WHs that allows a household to maximize energy and money savings for water heating while highly respecting end-user comfort. The proposed mechanism thus balances between the following objectives of load management: minimizing electricity consumption, minimizing heating costs and minimizing disruptions of user comfort. By finding trade-off(s) between the desired user comfort and the desired level of energy (money) savings, the model creates an appropriate water heating program for the WH for the coming day.

The rest of this chapter is organized as follows. We start with an overview of current challenges in electricity grids in Europe. In Section 1.2.1, we give an insight into the current energy consumption trends in European households, show the importance of water heating in the total energy consumption of a household and outline the potential of WHs as flexible loads. We briefly discuss DR of residential WHs and problems encountered in such applications in Section 1.3 and Section 1.4. Next, we discuss our research focus and state the problem tackled in this thesis in Section 1.5. The utilized approach and contribution of the thesis are outlined in Section 1.6.

\subsection{Challenges in Modern Electricity Grids}

With a rapid growth of the number of consumer loads (e.g., EVs) and expansion of RES, system operators are facing the problems such as peak demands and accommodation of less predictable and controllable distributed generation.

Electricity demand is changing all the time in the grid. If slow changes are more predictable and thus can be foreseen, rapid and sporadic changes of demand represent bigger risks. Peaks of the electricity demand can cause voltage drops which can adversely impact power system operation efficiency and security of the electricity supply [11]. Short voltage sags or long-term undervoltage can cause poor performance of consumer loads or even incorrect operation. The heavy drop of voltage may even induce rolling blackouts, if not timely handled. Power lines and electrical overloaded during the peak demand can easily get damaged, causing larger scale power outages. To restore the voltage of a gridpoint back to the allowed limits, grid operators perform real-time control actions that typically engage redistribution of reactive power in the grid by regulating of voltage on power stations, increasing the voltage on transformer of a local substation, switching on parallel segments of the grid, load shedding etc. Depending on the configuration of the 
grid restoration of the voltage after a grid failure can take from a few minutes to a few weeks.

Rapid spikes and drops of the electricity demand can create frequency imbalance in the grid. This is due to a change of speed of rotating turbines at the power stations. A rapid growth of the electricity demand can cause a mismatch between the mechanical and electromagnetic torques of the generation turbine, as a restult, the turbine rotations slow down and the frequency of the electricity supply also drops. Sudden drops of frequency in the grid can lead to disruption of work and downtime of consumer devices. The frequency regulation is a complex process that requires human involvement. For example, the primary frequency regulation implies stabilization of the rotational speed of a turbine by increasing the generating power. However, there is a risk that the required power generation will exceed the maximum allowed limits for the turbine, which can cause its damage. Also, a frequency regulation may require balancing of the power generation of more than one turbine at the power station.

Aside from the problems of reconciliation of the peak and rapidly (dropping) growing electricity demand, there are new challenges brought by a boost of decentralized generation (e.g., rooftop solar panels) and expansion of electrical vehicles. A growing number of people generating and storing energy from solar panels, home batteries and charging stations create challenges for energy transportation and dispatching because of difficulties in predicting the future balance of supply and demand emphasized TenneT, the TSO of the Netherlands [12]. Variable weather-dependent capacities of renewables such as wind farms and solar plants impede planning of generation assets as well. A mismatch between the planned and real generation, unpredicted consumption and failure of energy equipment can create energy imbalance in the grid and may cause power outages.

At the same time DSOs are facing a problem of integrating the rising shares of decentralized generation and new loads such as electric vehicles into their networks. Currently DSOs have to coordinate a variable and weather-dependent generation of prosumers, meter and account their power injections into the grid. In conditions of variable generation (e.g., weather conditions and self-consumption), it is becoming a cumbersome task to accurately predict the contribution of private RES into generation, bill and pay the customers and to maintain the distribution network on a reliable level. Spread of electrical vehicles as a highly mobile and thus highly unpredictable electrical load also represents certain risks for DSOs. In case, a big group of motorists all plug in their vehicles during the peak load time it can cause a network congestion.

The largest DSO in the Netherlands Alliander (35\% of all connections) has reported that the number of customers with decentralized energy production is rapidly growing in the operating region [13]. In 2016 the increase was $31 \%$ compared to 2015 and almost doubled from 2014. While overall customer satisfaction with the service has decreased by $3 \%$ in 2016 contrasted with 2015 . Such factors as the increased outage duration ${ }^{1}$ may influence consumer satisfaction.

Some grid operators argue for a price-driven (energy based rather than capacity based) electricity market that can host both centralized and decentralized energy production [12].

123.3 minutes in 2016 caused by a large low voltage disruption in Amsterdam in January 2016 against 21.9 min in 2015 


\subsection{Residential Water Heating and Electric Tank WATER Heaters}

The choice of residential water heating loads and in particular an electrical tank water heater $(\mathrm{WH})$ as a motivational example for our research is expounded in the following sections.

\subsubsection{Water Heating in Household Electricity Consumption}

Water heating accounted for $10.81 \%$ of total electricity consumed by the residential sector in Europe in 2017 [14]. Water heating is the second (14\%) most energy consuming and the third most electricity consuming (10.9\%) load in a European household as illustrated in Figure 1.2 in Figure 1.1.

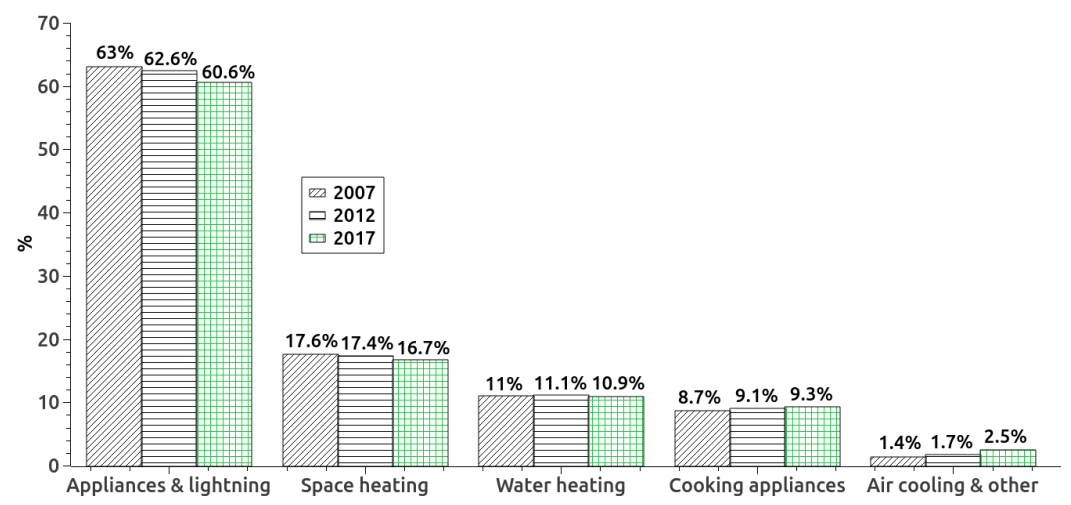

Figure 1.1: Household electricity consumption by end-use in the EU (based on [14]).

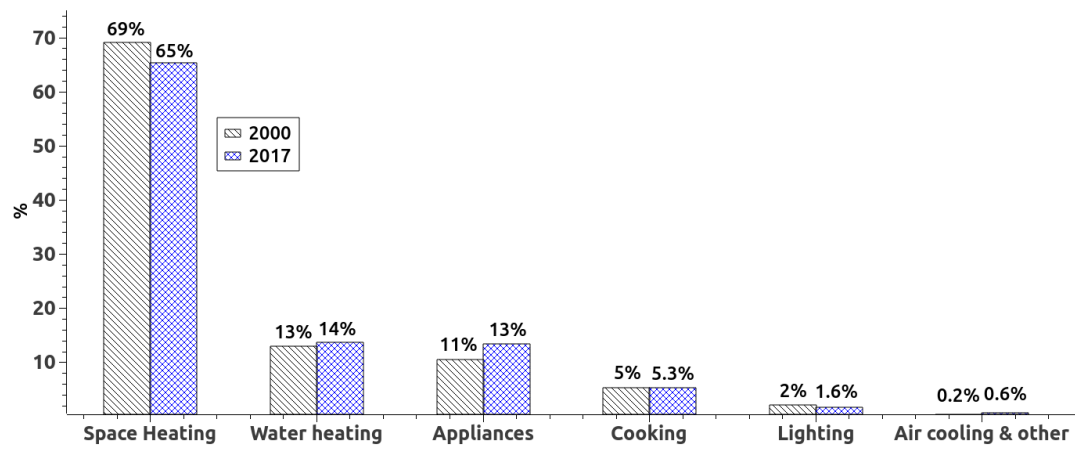

Figure 1.2: Household energy consumption by end-use in the EU (based on [15]).

It has been found that single family houses consume by $19.4 \%$ more energy for water heating than multi-family houses [16]. The EU project [17] predicts that the heat demand 
of European buildings is expected to decrease in the future because of a renovation of the current building stock, whereas the use of a domestic hot water is expected to increase.

As one of the most energy demanding end-uses in a household, water heating deserves special consideration. Water heating systems can have different configuration, equipment efficiency, fuel use and pipe insulation. There are different ways to configure a domestic water heating system. The two most popular ways are the tank recharge and instantaneous water heating [18]. Other configurations include mixed energy systems that use electricity combined with other energy sources (e.g., gas) to produce hot water also coupled with space heating and(or) solar collectors. The water heating usually takes place at one central point, however, sometimes there can be multiple separate heating units at home (e.g., a small tank heater for the kitchen).

The breakdown by fuel of the residential energy spent on water heating in 2017 is represented in Figure 1.3. The chart indicates that the biggest fraction of European domestic water heating is powered by gas, while electric water heating accounts for the second biggest share $(\approx 20 \%)$.

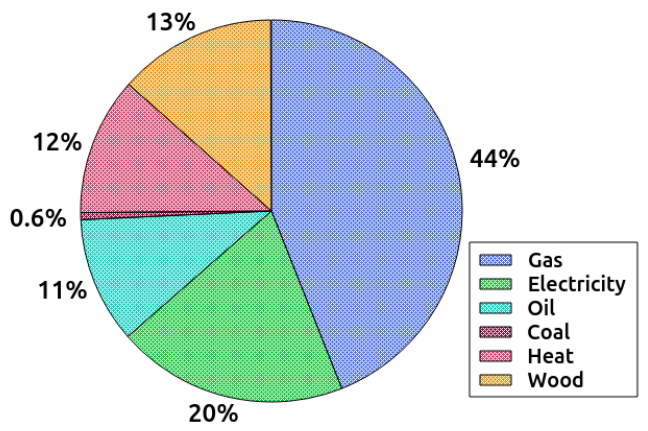

Figure 1.3: Energy consumption for water heating per fuel in 2017 (based on [14]).

The total number of water heating systems in Europe amounted to 218913 in 2015 of which $42 \%$ were gas installations, $12 \%$ electric, $13 \%$ biomass, $12 \%$ oil, $4 \%$ coal and $1.8 \%$ heat pumps. The same year, the biggest percentage of households that used electricity to heat the water was observed in Malta (60\%), France (33\%) and Greece (28\%).

As can be concluded from the above figures, water heating is a large contributor in the electricity consumption of households in Europe. Therefore, by increasing the energy efficiency of domestic water heating, it will be possible to influence the total electricity consumption of the residential sector.

\subsubsection{Park of Electric Tank Water Heaters}

Electric tank water heaters are present in a big number of European households. Over 89 million EU dwellings (36\% of total) used dedicated water heaters (not combined with space heating) as a primary source of sanitary hot water in 2014. Beside, around 53 million secondary dedicated water heaters used as a single tap solution (e.g. for the kitchen or 
bathroom sink). The estimates of the residential stock of primary water heaters showed that the share of WHs accounted for $64.1 \%$ ( $\approx 57$ million units) of all installations for primary water heating ${ }^{2}$ in Europe in 2014 [19]. Which accounted for $23.3 \%$ of the European park of water heating installations (including district heating, indirect and combi cylinders) in that year. The share of secondary WHs in the residential stock of secondary water heaters was $62.2 \%$ ( $\approx 33$ million units) in 2014. The sales of electric storage heaters are slightly on the increase, with a global trend to higher volumes. The sales of WHs in the EU in 2016 accounted for $71.1 \%$ and $36.8 \%$ of all electric water heaters ${ }^{3}$ and all installations for water heating, respectively.

\subsubsection{Water Heaters as Flexible Loads}

Electric tank water heaters represent "flexible" loads, meaning that energy demand of the WH can be interrupted for some period without a denial of its hot water service. This makes it possible to schedule the water heating periods of the WH more flexibly than in case of the conventional deadband control.

Similar to thermal energy storages, WHs can be viewed as devices that convert electric energy into heat, store and supply it to the end-user on demand. WHs have much smaller size and do not allow to efficiently convert the accumulated heat back to electricity. As such, WHs cannot be really used for re-electrification purposes and thus cannot be applied to provide electricity backup during periods of high energy demand. Instead, WHs represent a backup of hot water and allow to decouple the electricity demand curve for water heating from the hot water demand curve on a household level. Acting as buffers for thermal energy, they provide possibilities for load shifting. This can be for example used for flattening the household power demand curve. Electric tank water heaters are therefore commonly viewed as a good example of residential loads to be enrolled in demand response programs.

\subsection{Demand Response of Water Heaters}

The topic of DR of residential WHs is rather mature and has been rigorously studied over decades [20-22]. A detailed review of existing DR solutions for WHs is presented in Section 2.2. All the approaches for DR of WHs can be categorized into incentive-based and price-based.

\subsubsection{InCENTIVE-BASED DEMAND RESPONSE}

In incentive-based DR programs, electrical energy companies deal with shaping of the power demand of groups of consumer WHs to achieve peak demand shaving, load balancing, voltage control, frequency regulation, maintenance costs reduction, etc. The central goal of incentive-based programs is to schedule consumer WHs by turning them off and on at appropriate time slots so that the load regulation objectives are met. Activities related to incentive programs comprise direct load control (DLC), interruptible/curtailable load management (LM), voluntary load shedding and other $[9,23]$. Incentive-based programs are exercised on the contractual basis, meaning that residential consumers sign the contracts

\footnotetext{
${ }^{2}$ Other water heater types include gas instantaneous, gas storage, electric instantaneous, solar and heat pump water heaters.

${ }^{3}$ Electric water heaters also include instantaneous heaters and electric showers.
} 
with their utility companies based on which they agree to reduce their electricity demand to predefined values at agreed times ${ }^{4}$ in return of financial rewards. The benefits for consumers usually take the form of direct incentive payments and rate discounts. However, participants who do not respond can face penalties, depending on the program terms and conditions.

Thanks to incentive-based programs the system operators can operate pools of subscribed consumer WHs in order to mitigate grid congestions during the peak demand periods or when the grid electrical equipment fails. In well-established DLC programs, utility companies can remotely shutdown WHs of their customers on a short notice. When the load reduction event is over, the utility company restores the power supply of the disconnected WHs.

\subsubsection{Price-based Demand Response}

These days, energy companies are furnishing tariffs where the cost of electricity can vary depending on the energy system conditions in order to encourage consumers to move their electricity usage outside the periods of high prices, thereby reducing the load on the grid. Variable pricing reflects the energy system conditions ${ }^{5}$ which have to be maintained by system operators in order to supply the electrical energy in the demanded quantity and quality to consumers. Some of the widespread variable price tariffs are time-of-use (ToU), real-time pricing, variable peak pricing and critical-peak tariffs. For example, ToU tariffs typically apply to usage over long time intervals in which the price is determined beforehand and remains constant (e.g., day-night tariffs) [25]. On the contrary, in realtime pricing, prices are determined close to the real time electricity consumption (e.g., 15 minutes) and are linked to wholesale electricity market prices. In price-based DR, consumers can voluntary react to the variable prices (no commitment) by shifting their energy consumption to periods of low prices. As such, consumers can benefit financially while at the same time reducing the stress on the grid.

The key differences between incentive-based and price-based DR are summarized in Table 1.1.

Technical implementation of DR of WHs requires means such as communication links between a utility company and consumer, and a home energy management system (or a controller) on the consumer side that can respond to the steering signals (load adjustment messages or pices) and automatically plan and modify the heating periods of the WH with respect to consumer's and(or) utility company's goals. Making a decision to alter the hot water demand or operation of the $\mathrm{WH}$ in response to a changing electricity price might be a non-trivial exercise for a consumer, especially if the price is changing in close to the real-time fashion (e.g., every 15 minutes). This task usually pertains to estimation of the profits from changing the energy consumption and potential impacts of such changes on the user comfort. A home energy management system (or controller of a WH) can assist consumer in choosing and implementing the best options for planning water heating

\footnotetext{
${ }^{4}$ For example, load reduction events can take place from Novermber to March between 1-11 PM with maximum duration of 5 hours [24].

${ }^{5}$ In deregulated energy markets, wholesale and clearing market electricity prices are calculated by system operators based on the energy-to-price bids submitted by generating companies (including importers) and load servicing parties (including electricity consumers and exporters) and transmission network limitations.
} 
Table 1.1: Key differences between incentive-based and price-based DR.

\begin{tabular}{|c|c|c|}
\hline & $\begin{array}{l}\text { Incentive- } \\
\text { based }\end{array}$ & Price-based \\
\hline Control initiator & $\begin{array}{l}\text { utility } \\
\text { company }\end{array}$ & consumer \\
\hline Steering signals & $\begin{array}{c}\text { load } \\
\text { adjustment } \\
\text { messsages }\end{array}$ & market price \\
\hline Consumer stimulus & $\begin{array}{c}\text { direct } \\
\text { payments }\end{array}$ & variable price \\
\hline Contract obligations & $\checkmark$ & $x$ \\
\hline Consumer penalities & $\checkmark$ & $x$ \\
\hline $\begin{array}{c}\text { Guaranteed consumer } \\
\text { rewards }\end{array}$ & $\checkmark$ & $x$ \\
\hline $\begin{array}{l}\text { Guaranteed benefits for } \\
\text { utility company }\end{array}$ & $\checkmark$ & $x$ \\
\hline $\begin{array}{c}\text { Consumer loads are } \\
\text { dispatchable resource for } \\
\text { TSOs/DSOs }\end{array}$ & $\checkmark$ & $x$ \\
\hline $\begin{array}{l}\text { Market access via } \\
\text { aggregator }\end{array}$ & $\checkmark$ & $x$ \\
\hline
\end{tabular}

$\S$ Consumer can participate in wholesale, balancing and ancillary services markets through the services of aggregators [6].

periods, can allow to account for user preferences, can provide billing information and statistical data on electricity usage, etc.

\subsection{Challenges of Demand Response}

\subsubsection{Consumer Acceptance of Demand Response}

The adoption of DR in practice balances in-between how consumers perceive possible benefits and shortcomings. In spite of the multiple benefits that DR brings to consumers, the majority of DR participants today is typically represented by volunteers, but not by all consumer groups in general [8]. As it has been highlighted in the EC Task Force for Smart Grids "the engagement and education of the consumer is a key task in the process as there will be fundamental changes to the energy retail market" [26]. The EU is concerned about adaption of DR advances, i.e. "...whether consumers will embrace the new technology..." [27].

Currently there is still a significant level of consumer resistance to participation in DR, mainly because consumers are afraid of losing control of devices in their households and are sceptical about new electricity rates [28]. The studies of Joint Research Centre shed a light on the main factors that determine consumer involvement in DR [8]. Factors such as energy bill reduction, consumer concerns about environmental impact and better comfort expectations play leading roles in consumer acceptance of DR. Although energy suppliers 
increasingly use the environmental factor as a motivational incentive (e.g., renewable or green energy contracts) to get the customers involved, a recent survey done in the U.S. shows that consumers also put a high value on personal comfort [10].

Flexible consumers driven by objectives to save electric energy/money, to reduce their environmental footprints, to support the lack of electricity generation during complex operational conditions are often unaware about the exact profits they can gain by practicing DR as well as about implications of DR on their comfort. It has been proven that unpredictable impact of DR control actions on occupants comfort impedes residential consumer uptake of DR solutions. Ill-conceived control actions taken without regard for consumer preferences can deteriorate consumer comfort so that DR participants might decide to sign out.

\subsubsection{Impacts of Demand Response of Water Heaters on User COMFORT}

Demand response (DR) of WHs modifies a regular operation of the WH according to goals of a utility company (e.g., peak load reduction) or a consumer (e.g., lower electricity bill). However, the resulting changes in the water heating profile may lead to cooler tank water temperatures than those required by the end-user during hot water usage periods, thereby DR may negatively impact end-user comfort.

The end-user may agree to accept lower water temperatures during specific periods or may not depending on the urgency of hot water activities, the timing of the cold water periods, individual tolerance to cold water, etc. Albeit, if residents have to significantly alter their hot water consumption habits and endure less comfortable water temperatures this may bring them inconvenience. The increasing dissatisfaction with the suggested water heating can outweigh the desire to benefit financially (or to lower environmental footprints) so that the consumer may reject the benefits of DR [8, 29-31].

\subsubsection{Ene rgy Efficiency of Water Heaters in Demand Response}

There is a numerous number of existing solutions for incentive-based [20,32, 33] and price-based DR of WHs [34-36]. Most of the solutions ensure user comfort by keeping the tank water temperature above a predefined minimum threshold [37-39]. That minimum threshold is typically chosen above $50-60^{\circ} \mathrm{C}$ in order to: (a) provide user comfort comparable to the case of unmodified operation of the WH (so-called deadband control), (b) prevent contamination of the WH tank by harmful bacteria Legionella [40]. Some solutions allow for lower setpoint temperatures, for example, $45^{\circ} \mathrm{C}$ [41]. As a result, the tank water often remains above the room temperature (e.g., $24^{\circ} \mathrm{C}$ ) at times when no hot water is used which means that the thermal energy is stored in the WH not optimally because of the heat losses [20,37].

Traditionally, DR is more concerned about the desired shaping of the electricity demand curve during a day, i.e. matching the power demand of consumers with generation power of an electricity supplier. Which entails changes of the energy consumption times (scheduling in time) of the $\mathrm{WH}$, but not changes of its energy consumption levels. The questions such as what amount of the heat losses delivers the control and whether or not the heating is optimal in terms of the heat losses are barely discussed in the literature. 


\subsection{Research Scope and Problem Statement}

The research presented in this thesis aims at DR solutions for residential consumers to enable energy and(or) money savings while highly respecting occupants' comfort.

The goal of this research is to investigate how control mechanisms for WHs can overcome the problem of possible user comfort degradation in DR of residential WHs. Furthermore, we aim to explore the control possibilities to increase the energy efficiency of the $\mathrm{WH}$ without an impact on the end-user comfort. This research focuses on new control strategies and algorithms for domestic WHs that allow for simultaneous satisfaction of (sometimes) conflicting objectives of maximization of consumer profits ${ }^{6}$ and minimization of end-user comfort disruptions. In contrast to utility company-driven incentive-based DR solutions, we concentrate on user-oriented control mechanism where control of the WH is initiated by the consumer himself. Our research focus is on the level of a single household and a single WH unit.

Taking a domestic WH as an example of water heating loads, our research pursues the following goals:

1. increase the energy efficiency of hot water supply in households;

2. bridge the gap between the consumer's objectives of energy/money savings for water heating and end-user comfort.

\subsubsection{Energy EFficient Control}

Unlike conventional DR that only deals with shaping of the power demand curve, our research focuses on control that optimizes the energy efficiency of the $\mathrm{WH}$, i.e. when the WH consumes less electricity providing the same quality of hot water service to the user(s). The most straightforward way to reduce the amounts of energy consumption of the $\mathrm{WH}$, i.e. achieve energy savings, is to reduce the heat losses by lowering the operational tank water temperatures at right times.

It is obvious that zero heat losses mean that the tank water temperature is equal to the ambient air temperature which typically is too low to fulfill all hot water activities without negative impact on the user comfort. In other words, the goals of minimization of the electricity consumption for water heating and minimization of user comfort disruptions are conflicting objectives in the context of hot water activities. Therefore, the task of improving the energy efficiency of the WH control should be accompanied by the task of the user comfort satisfaction.

In general, the reduction of the energy consumption as a standalone objective can open the gates to minimization of the environmental footprints and maximization of the system reliability on the community level. Furthermore, by reducing the levels of energy consumption for water heating a consumer can benefit financially, even if his household operates under a flat electricity tariff and no DR is implemented. Moreover, the idea of controlling energy consumption instead of only power demand also supports the course toward the energy-based electricity markets where players trade amounts of energy and flexibility to reduce energy consumption rather than operate with capacities [12].

\footnotetext{
${ }^{6}$ The energy savings achieved by the improved efficiency of the WH can be theoretically converted into money equivalent and thus referred here as profits.
} 


\subsubsection{USE R Comfort SATisfaction}

The second problem tackled in this thesis is how to reconcile (sometimes) conflicting objectives of energy/money savings with the objective to maintain a user-desired level of comfort during the hot water usage.

As discussed in Section 1.5.1, the goal of improving the energy efficiency of the WH control comes along with the necessity to ensure acceptable level of user comfort. Furthermore, the price-based DR presupposes maximization of money savings by shifting the WH load to the low-price periods during a day. In case the destination low-price time slot is too distant in time from the actual hot water usage, such load shifting might lead to cooler tank water temperatures than those required by the user (heat losses) and cause user discomfort.

One can draw a conclusion that the necessity to account for user comfort in control of WHs is dictated by two reasons: (1) to perform control with a full customer satisfaction and (2) to implement the control on the edge of the user comfort so that the electricity is consumed at the minimum yet acceptable to the user comfort level.

\subsubsection{RESEARCH QUESTIONS}

The research questions covered by this thesis can be summarized as follows:

- How to quantify user satisfaction with the hot water service at home?

-What is the optimization potential of WHs with regard to energy and money savings?

- Is it possible to increase the energy efficiency of the $\mathrm{WH}$ operation without a decrease of the end-user comfort by means of control?

- Can the price-based DR of WHs be implemented without deterioration of the user comfort?

\subsection{Approach And Contribution}

In respect of the research questions, this thesis contains the following contributions:

- hot water activities framework. In our research we utilize an activity-based approach for modeling user satisfaction with the hot water service at home. In this approach, household daily hot water demand is viewed through the lenses of hot water activities (or events) that have a set of attributes associated with them. Thus, each hot water activity (WA) during a day has a start and end times, a user-desired tap water temperature and water flow rate. We argue that occupants can sometimes tolerate cooler tap water temperatures and(or) tap water flow rates, and that such tolerances can be exploited in control of the WH. Therefore, besides the aforementioned attributes, WAs can have the user temperature and flow tolerance (comfort) zones, wherein each value is experienced by the end-user as equally comfortable.

- user comfort model. To quantify user satisfaction with the hot water service, we introduce a user comfort model. The user comfort model includes the environmental variables (e.g., the tap water temperature) and personal variables that express individual preferences for the environment (e.g., the temperature comfort zone). The link 
between these two types of variables is established via user tolerance functions that model how variations of the environmental variables influence the user feeling of comfort with regard to personal preferences. In other words, the tolerance functions reflect the real user feeling of the environment and quantify it.

- energy model. To explore the optimization potential of domestic WHs with regard to the goal of increasing energy efficiency (energy savings), we developed an optimization energy model. Our optimization approach for WHs is built upon the proposed user thermal comfort model. The energy model thus allows for scheduling of the WH heating periods on a daily timescale so that the electricity consumption is minimized and the user thermal comfort is maintained at the user-desired level.

- price model. In order to address the question whether or not the price-based DR of WHs can be implemented without end-user discomfort, we designed an optimization price model that schedules the water heating according to the day-ahead electricity market prices. The price model performs the WH load shifting while simultaneously respecting the consumer goals to maximize profits from DR and to maintain the end-user comfort at the desired level.

\subsection{Thesis Organization}

In this chapter, we gave a brief overview of current trends in the European energy system, introduced the topic of demand response (DR), discussed the obstacles connected to practical implementation and consumer acceptance of DR and outlined the motivation of our research. We defined the focus of our research, formulated our research questions, showed the outline of our approach and presented the contributions. The remainder of this thesis is organized as follows.

We start Chapter 2 with an introduction of the hot water system setup under consideration. Next, we provide an overview of the state-of-the-art solutions for DR of electric tank water heaters. Based on the comparison of different existing approaches, we emphasize the necessity of further comprehensive modeling of user comfort during hot water usage. In the end of Chapter 2, we discuss different possibilities for modeling the WH operation and justify our choice of the thermodynamic model of the WH. Chapter 3 presents our approach for user comfort modeling. We elaborate on essential variables and properties of the hot water usage to be accounted for in user comfort modeling and propose our user comfort model consisting of the user thermal comfort model and user flow comfort model components. In Chapter 4, we explore different control strategies that allow to maintain/improve the thermal comfort of the end-user for the case of a single hot water activity (WA). These control strategies are extended for the case of multiple WAs in Chapter 5. Chapter 5 deals with the problems of a fair reconciliation of the conflicting objectives of (i) minimization of energy consumption for water heating and minimization of the end-user thermal comfort disruptions and (ii) minimization of water heating costs and minimization of the user thermal discomfort. In that regard, we introduce the energy and price optimization models that allow for simultaneous satisfaction of the mentioned objectives and discuss how the proposed control mechanism can be implemented in practice. 



\section{2}

\section{RELATED WORK}

This chapter introduces the topic of control of residential electric tank water heaters (WHs). We give an insight into motivational factors for altering a regular operation of domestic WHs and provide an overview of state-of-the-art control solutions for WHs.

In this chapter the related literature on the topic is presented. The chapter provides a reference information on theoretical research and successful practices in demand response (DR) of residential tank electric water heaters (WHs), on modeling of the WH operation and on user comfort.

The chapter begins with a description of a considered domestic hot water system consisting of the WH and mixer taps connected by a system of pipes. We further delve into the topic of control of WHs with a specific focus on DR techniques. The goals of utility companies and consumers participating in DR are discussed next to each other. The overview of state-of-the-art solutions for DR of residential WHs is given with their separation into price-based and incentive-based types of DR. We analyze the inherent features of the solutions with a focus on the optimization techniques and objectives they pursue. The ways different solutions represent user comfort, model and control the WH operation are considered in detail.

We stress the need to account for and to minimize the WH heat losses in control algorithms for WHs as a main enabler of the energy efficient domestic hot water supply. The role of the user comfort and the necessity of its modeling and incorporation into control algorithms for WHs is discussed after that.

Because of the different possibilities to model the operation of WHs, we explore the pros and cons of a stratified and well-mixed WH tank models. We provide our justification for opting in favor of the well-mixed WH tank model in the current thesis. The well-mixed WH model utilized in this research is presented and different operational modes of the WH are modeled. Finally, we explain the choice of the parameters for the adopted WH model. 


\subsection{Hot Water System at Home}

A typical domestic hot water system consists of a water heating unit, a tap mixer and a cold water supply connected by a system of pipes, as shown in Figure 2.1.

Domestic electric tank water heaters (WHs) are heating units that use water storage tanks to maximize their heating efficiency. The water inside the water reservoir is heated by electric resistive heating elements $P_{\mathrm{e}}$. Hot water is released from the top of the water tank $P_{\mathrm{hw}}$ under the mains pipe pressure when the hot water tap is open. At the same time, that cold water enters the tank through the inlet at the bottom $P_{\mathrm{cw} 1}$ so that the volume of water inside always remains the same. Importantly, users seldom request water at high temperatures directly from the $\mathrm{WH}$, but rather use a mixer tap to obtain a cooler tap water temperature. The hot water flow from the $\mathrm{WH} P_{\mathrm{hw}}$ fused with the cold water flow $P_{\mathrm{cw} 2}$ produce the hot tap water $P_{\mathrm{d}}$ in the tap mixer. The WH tank dissipates the heat to the surroundings in the form of heat losses $P_{\text {loss }}$.

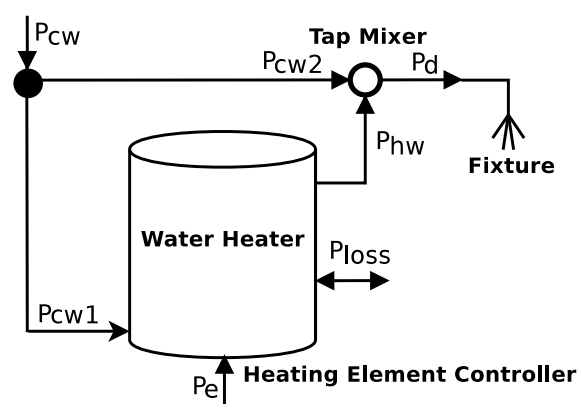

Figure 2.1: Typical domestic hot water system ${ }^{1}$.

Most residential WHs do not modulate their power, meaning that their heating elements are either switched off or turned on to the maximum power to maintain the tank water temperature within some temperature margin. This margin is determined by the lower and the upper setpoint temperatures of the WH thermostat. The heating element of the WH is turned on, when the water temperature is below the lower setpoint temperature inside the tank. Once the water temperature hits the upper setpoint, the heating elements are shut off and the process repeats. Unlike instantaneous (tankless) water heaters, the heating capacities of WHs are typically insufficient to maintain a stable tank water temperature during hot water usage. Which means that (a) the tank water temperature usually drops during the hot water usage, (b) the hot water is prepared before the actual usage and stored in the tanks at certain temperature setpoints.

The lower setpoint temperature $\left(60-70^{\circ} \mathrm{C}\right)$ is dictated by sanitary norms to prevent contamination of the WH tank by harmful bacteria Legionella [40]. The upper setpoint temperature is set based on manufacturer and safety hazard recommendations to attain an

\footnotetext{
${ }^{1}$ As it will be shown in this chapter, there are different ways to model the operation of the WH. The energy inflows and outflows of the WH in Figure 2.1 exhibit only the energy flows for the system "water-insulation-ambient air" (i.e., tank stratification is not present).
} 
efficient operation of the heater and to prevent the skin scalding [42]. These temperature settings prescribe the $\mathrm{WH}$ how much heat to accumulate and store.

\subsection{Control of Electric Tank Water Heaters}

Motivation of electrical energy companies to modify a regular operation of residential WHs can arise from a need to perform balancing of power generation and demand, voltage and frequency regulation. For example, unplanned large loads can cause system disturbances such as voltage drops which can impact security of the electricity supply and may cause power outages [11]. To prevent the power supply from halting completely provided that system voltage is decreasing, a grid operator can temporarily turn off residential WH loads during the voltage recovery period. Today, electricity providers promote Demand Response (DR) programs where subscribed consumers agree to respond to electricity demand reduction requests. In addition, energy suppliers nowadays encourage consumers to reduce electricity usage during certain periods of a day by offering them variable price tariffs.

The control objectives pursued by electricity consumers can differ from those of their electricity providers. From a consumer perspective, the objectives might be, for instance, a reduction of the electricity bill, contribution to the stability of the electricity grid, decreasing the environmental footprints, etc. For example, a consumer might want to benefit financially by subscribing his WH into a DR program where the WH is remotely shut down during the peak electricity demand periods on request of a utility company in return for incentive payments. Another example of a modified WH operation is a response to variable electricity prices. Under a variable price tariff a consumer may want to let his $\mathrm{WH}$ heat the water during the low-price periods thus reducing the heating costs. Moreover, some consumers these days aim at lesser environmental footprints which can be achieved by reducing their electrical consumption. Unlike shaping of the energy demand curve of WH loads, this objective is related to the reduction of the amount of the consumed energy. Besides the diminished impact on the environment, the reduction of the electricity consumption yields financial benefits for consumers.

Approaching the Demand Response objectives becomes possible by incorporation of additional steering signals in the control of the WH. Such steering signals can come from the outside world (e.g., electricity prices) and(or) from other consumer devices (e.g., the output voltage of a solar panel). The central idea is to heat the water by reacting to the steering signals so that the regulation objective(s) are maximally satisfied. The new control of the WH might be initiated by a remote party as in case of incentive-based DR (e.g., load curtailment programs) and(or) by a consumer himself in response to the changing electricity price.

Residential WHs have been traditionally considered as suitable candidates in many load incentive-based DR applications [20, 32, 33, 43]. The topic of WH load shifting in response to variable price signals is also broadly covered in the literature [34-37].

\subsubsection{InCentive-based Control of Water Heaters}

In incentive-based DR programs, electric utility companies initiate control of WHs of their customers in order to achieve objectives such as peak demand shaving, load balancing, 
frequency regulation, voltage stability, maintenance costs reduction, etc. Such control can be seen as an identification of the best times to initiate the WH heating/cooling periods. The heating/cooling periods can be initiated by switching on/off the WH heating elements and by changing the thermostat setpoint temperatures. Another less widespread technique is to control the voltage supply of the $\mathrm{WH}$ without its disconnection from the electricity grid [32, 44, 45]. Some examples of incentive-based load management (LM) approaches for WHs are present below.

A LM algorithm for shifting WH loads to off-peak load hours was proposed by [20]. The algorithm aimed at minimization of the electricity consumption of WHs during the peak demand periods while minimizing the potential user discomfort caused by the load reduction. The problem was formulated as a dynamic programming (DP) optimization problem where on every time interval of a discrete timescale two objectives were simultaneously minimized for all the following intervals. More specifically, the objective function was represented as a weighted sum of two objectives across all the intervals inside the planning horizon. The first term of the objective function weighted with a power penalty cost coefficient represented the cumulative energy consumption on all the following discrete intervals and thus respecting the goal of load reduction. While the second term weighted with a comfort penalty cost coefficient was formalized as a cumulative difference between the actual tank water temperature and the predefined temperature setpoint on all the coming intervals, and was responsible for a user satisfactory hot water in the $\mathrm{WH}$ tank. Both the weighting coefficients were functions of time. The target groups of WHs to implement the devised control were selected based on a clustering approach.

A new LM strategy for micro grids with higher penetration of WHs was proposed by [32]. The goal was to reduce the peaks in power demand, power rampage and energy costs by means of the voltage control of WHs whenever required by a utility company. The idea of control was to suppress the voltage of the power supply of the WH during peak demand periods while providing the required voltage conditions to other household loads. The suggested AC electric springs coupled in series to the WH load and critical loads allowed for regulation of the WH voltage supply (affects power demand of the WH) while supporting other loads with reactive power. The proposed user-defined energy management algorithm was minimizing the absolute difference between the active power demand in adjacent hours based on the percentage of power savings given by a user. The user-specified reduction of the power demand was achieved by altering the WH voltage (e.g., in the range $180 \ldots 220 \mathrm{~V}$ ) supplied using electrical springs.

A solution to allow WHs to participate in frequency regulation was proposed by [33]. The idea of control was to store energy in compliance with the tank water temperature setpoints and the grid electricity frequency. When the grid frequency was high, the controller increased the power of the lower heating element if only not overruled by the upper temperature setpoint, if the frequency dropped the power of the heating element was reduced with respect to the lower thermostat temperature settings. The user comfort was ensured by the thermostat controller that was tracking the temperature deviations and by letting the upper heating element of the WH operate as usual, i.e. only the lower heating element was controlled.

An attempt to reconcile the multiple objectives such as the peak load reduction, energy consumption and user comfort was made by [43] by means of a centralized mechanism for 
peak demand management of WHs. The authors specifically focused on a solution for a configurable load threshold (as by power level as well as by the time of occurance) set by a utility company. The algorithm aimed at limiting the peak demand of a group of WHs while providing the satisfactory user comfort and reduced heating costs by controlling each individual WH. A heuristic approach based on the urgency metric was chosen to decide with which of urgency each WH had to be heated. The urgency metric was defined by a cost function consisting of two terms (i) the time before the next water usage and (ii) the amount of heating time needed to heat the water up to a predefined setpoint temperature. Thus, the term (ii) of the cost function respected the user comfort. The metric was then evaluated by a centalized controller to make a decision about the WHs that had to be turned on or off at every time step. The impact of control on user comfort was evaluated through the weighted mean water temperature represented by tank water temperature, event hot water duration and volume of hot water used during the hot water event.

Two reinforced learning techniques for multi-objective load management of multiple WHs were explored in [46]. The goal was to find the on/off schedule of the WH that can minimize the load demand during peak periods as well as the total energy consumed while satisfying customer comfort. In the proposed approach, each WH was considered to be an artificial agent that was first trained to adapt based on hot water consumption and grid load demand profiles and then operated according to the found policy. The reinforced learning techniques based on Q-learning utilized the following information: (i) the tank water temperature, (ii) the hot water consumption, and (iii) the energy price (or the grid load). The possible combinations of the input variables (i)-(iii) were fuzzified into membership functions to obtain the relationship between the system state (output) and its input. The controlled (decision) variable represented the state of the heating elements of the $\mathrm{WH}$. The reported silumation results showed the reduction of the energy costs under ToU tariff while reuducing the energy consumption during the peak demand hours.

Three decentralized control strategies for scheduling the WHs based on price, load and voltage control were presented in [44]. While the price-based strategy was more consumeroriented, the other two were forged to provide support to the low voltage distribution grid in terms of load reduction and voltage control, namely loading-based and voltage based control strategies. Working on the level of a single $\mathrm{WH}$, the loading-based control aimed at providing technical grid support during peak demand or critical periods by shifting the WH to off-peak hours. Finally, the voltage-based control was supplying voltage suport to the grid by turning off the WH in case of the voltage drops. The user comfort was ensured through the minimum and maximum thresholds of the stored energy.

A scheduling approach for load reduction of a large population of WHs was presented in [41]. The tackled problem was to find the number of WHs to be turned on and off in order to achieve the desired load threshold in a given time frame. To solve this problem, the objective was formulated as minimization of the difference between the number of WHs that fits the energy consumption specified by the demand threshold and entire time frame and the number of WHs to be scheduled for on/off actions in a smaller fractions of that period. The considered constraints accounted for the balance between the power generation and demand, thermal energy storage limits of WHs, the energy balance and tank water temperature constraints. The distinguish features of the proposed approach could be identified as (i) the random temperature (or energy) distribution of WH groups 
was modeled by histograms explained by the devised discrete time and state model and (ii) a broad tank water temperature range $\left(45-65^{\circ} \mathrm{C}\right)$ was considered for random distributions of WHs. The user comfort was respected by a rule that prohibited switching off the WH if its tank water temperature was in the tepid zone $[15,45]^{\circ} \mathrm{C}$.

Another peak reduction algorithm for WHs based on proportional and integral methods was presented by [47]. To obtain the optimized switching program, the algorithm iteratively minimised the mean error between the target load reduction value set by the user and the mean controlled load in each switching cycle of a switching program. More exactly, the optimization iteratively summed errors of switching off the group of WHs on each control interval, multiplied it by proportional gain/duration coefficient of the current switching off period and created a new switching off period the $\mathrm{WH}$ for the next interval.

A set of LM algorithms for household peak reduction was examined by [48]. Two algorithms for multiple household loads including WHs implemented different load shifting policies by operating on/off states of devices. The first algorithm moved the loads of flexible devices (e.g., WHs, washing machines) outside the peak demand periods. Another algorithm aimed at optimizing the on/off switching times across the devices with regular on/off cycling (e.g., WHs, refrigerators, air conditioners) so that the gaps (inactive periods) of one device were filled by load (active) periods of other devices. The study specifically explored the effect of three LM algorithms for multiple household loads including WHs on human comfort. The least-to-highest potential user inconvenience ranking was identified for algorithm that coordinates the timing of loads with constant cycles (e.g., WHs) and load shifting to off-peak hours. A non-uniform time buffering between the power demand of a single WH and the hot water service provided to the user was identified as a major factor determining the ability to shift the WH load. The impact of control on user comfort was evaluated through a prism of "urgency" of the hot water service to a user. That is to say, the user discomfort pertained to the waiting time between the moment the hot water with a user-desired temperature was requested by and delivered to a user.

An algorithm to reduce the peak hourly load of multiple household electrical devices including WHs was developed by [49]. The control algorithm was suitable for scheduling of power-shiftable loads (e.g., WHs), time-shiftable loads (e.g., a washing machine) and nonshiftable loads (e.g., an over). in home energy management systems (possibly, applicable for home areas). The integer programming algorithm was aimed to minimize the total hourly load of appliances by scheduling their switching on/off patterns under a set of constraints specific for each type of loads. The constraints applicable for WHs were imposed as in the form of box constraints for their load to not exceed their maximum working power and not be lower than their stand-by loads. Comfort was regarded through the time-varying constraints on the WH power demand.

An algorithm for appliance commitment problem of household loads including WHs was proposed by [50]. The tailored algorithm focused on the peak load reduction of the aggregated household load while maintaining the user comfort and the energy costs on the acceptable to users levels. The user comfort related to the WH was modeled by piece-wise functions that reflect user convenience levels depending on the tank water temperature values of the WH. The problem was formulated as min-max optimization problem where the minimization of the user discomfort coming from an adjusted power demand of loads was accompanied by the maximization of the total load of appliances to be lower than 
some predefined threshold introduced in the form of the constraint. The algorithm was choosing trying to lower the energy consumption or to shift the appliance that causing the lowest impact on user comfort.

A control mechanism for aggregated peak demand reduction of WHs with two heating elements (upper and lower) was proposed in [51]. The mechanism for every WH consisted of two switches and a lower thermostat in addition to the conventional upper and lower WH thermostats. These switches were responsible for implementing the on/off signals for load reduction coming from a utility company, while the task of the additional thermostat was to account for the minimum allowed tank water temperature setting. The control operated the on/off state of only the lower heating element of the $\mathrm{WH}$ without changing the regular operation of the upper heating element in order to satisfy user comfort. The central controller located at the utility company monitored the on/off states of the lower heating elements and the additional thermostat. In case the decrease of the electricity consumption of the WH was required the central controller disconnected the lower heating element of the $\mathrm{WH}$, the central controller sent the signal to account for the minimum allowed temperature setting by connecting the additional thermostat if it was required to increase the WH electricity consumption. A detailed thermodynamic model of the WH was applied to account for both thermodynamics and user comfort.

A multi-objective optimization algorithm for a group of WHs was introduced in [52]. The proposed binary particle swarm optimization algorithm applied to multiple WHs allowed for balancing between the objectives of minimization of the aggregated peak load demand and maximization of the user comfort. The peak demand reduction was achieved by searching for the best on/off schedules of WHs so that their aggregated demand was as close as possible to the desired threshold. On the other side, the user comfort was simultaneously maximized in a single objective function by minimizing the difference between the maximum and the measured tank water temperatures of WHs. The user comfort was modeled as a difference between the maximum WH setpoint and the tank temperature estimated based on the thermodynamic model of the $\mathrm{WH}$.

A fuzzy logic controller for centralized peak demand reduction of a group of WHs was presented in [21]. To shave the aggregated peak demand to times preceding the peak demand, a pre-heating control strategy was employed, i.e. the upper thermostat setpoint was raised to a higher value before the peak demand period and the WH was then shut off during the peak period. In a discrete time fashion, the controller fuzzified the inputs such as the aggregated load demand from the power distributor and the expected load profiles of groups of WHs after pre-heating in order to decide which groups should be shut down. The comfort preferences were represented by the fuzzified membership functions based on historical WH power demand profiles classified into groups with the same water usage behavior and combined with the average minimum and maximum thermostat setpoints.

An approach for identification of on/off patterns of thermostatically controlled loads, such as WHs, in direct load control programs was formulated in [53]. The goal was to find fair solution that simultaneously satisfies multiple (possibly conflicting) objectives such as the minimization of the maximum power demand, maximization of profits, minimization of the electricity grid power losses, and minimization of causing discomfort of control to consumers. A combinatorial multi-objective optimization (CMOO) approach was applied to reconcile these objectives. To find the the best (non-dominated) solutions of the CMOO 
problem, an evolutionary (genetic) algorithm was proposed. The evolutionary algorithm applied genetic operators (selection, crossover and mutation) to create new populations of binary on/off solutions for groups of loads while estimating their effectivness based on a decision maker's preference information. Considering the WHs, consumer discomfort was accounted for by two time-related indicators, namely the total time the tank water temperature below or above a specified threshold and the maximum period during which this situation occured.

\subsubsection{Price-based Control of Water Heaters}

Similar to incentive-based load management of WHs, price-based control boils down to identification of the best times to initiate the water heating/cooling periods of the WH. In contrast to incentive-based approaches, price-based control is performed in accordance to a variable electricity price signal. Most of the existing strategies for price-based scheduling of residential WHs are consumer-oriented, meaning that they specifically aim at satisfaction of the consumer goal to benefit financially from load shifting. We present some examples of such variable price-based scheduling of WHs below.

A control algorithm for scheduling of a single WH under variable pricing was proposed by [34]. The algorithm aimed at minimization of the electricity bill of a single household without incurring a high discomfort for the end-user. Thus, the objective was formulated as simultaneous minimization of the energy and user discomfort costs. In the suggested approach, the controller performed the WH load shifting by postponing the energy volume to the future time intervals without regarding for the tank water temperature, i.e. the model was fully based on the energy volume and time. The optimization model utilized the input information about three time-related parameters that reflected the user readiness to reduce his comfort (i) the periods not allowed for WH load disconnection, (ii) the maximum disconnection time, and (iii) the minimum time between two disconnections. Besides, the model used the input information about the expected energy consumption of the WH from the forecast, electricity prices and the price for activating the flexibility of load shifting. The user discomfort was represented in the form of costs or the savings in the electricity bill needed for activating the WH flexibility. These costs were formalized as the weighted average delay between the forecasted and shifted energy consumption times the price for activating the flexibility.

A multi-objective optimization approach was applied by [35] to simultaneously minimize the heating costs and possible consumer discomfort from $\mathrm{WH}$ scheduling. The weighted sum approach was applied for solving the multi-objective problem. More specifically, the objective function was represented as a weighted sum of two functions, representing the heating costs and user comfort satisfaction. The non-linear thermodynamics of a well-mixed WH model was represented in a form of piece-wise linear constraints for the tank water temperature. In addition, a constraint for not letting frequent on/off control actions on the heating elements of the WH was introduced in order to decrease the impact on the WH life-time. The authors accounted for the presence of the tap mixer and represented the user comfort as a cumulative difference between the supplied tap water temperature and the user-desired tap water temperature (similar to [54]). The mixed integer linear problem thus contained a set of linear variables that represented the tank water temperature, and the set of binary variables representing the on/off control actions 
and auxiliary variables.

An interval-based approach to deal with uncertainties in hot water demand, ambient temperature, and real-time price of electricity was employed by [36] for WH scheduling. The model predictive control (MPC) algorithm based on the forecasts and newly updated information was devised for minimizing the electricity bill of a consumer. The well-mixed tank thermal dynamic model of the WH was transformed into an interval notation, where the tank water temperature real value at each interval was represented by a random variable with a uniform distribution between its lower and upper bounds. Besides the tank water temperature, other uncertain variables like the hot water demand, the ambient temperature, the real-time prices and the user comfort zone for tank water temperatures were also represented through the interval variables. The original problem to minimize the heating costs on the planning horizon based on uncertain interval-valued price variables and subject to uncertain temperature and comfort constraints was translated into an unconstrained probabilistic optimization problem. More exactly, minimization of interval-based heating costs was represented via minimization of the weighted sum of its deterministic middle point and radius where the weights meant the desire of the user to focus on either minimization of the mean price values or the radius of the energy costs. Further, the tolerance degree for constraint violation was introduced to transform the uncertain user comfort constraints into a probabilistic function incorporated in the objective function with its own penalty factor. Thus the user comfort was represented in a more flexible fashion rather than just a hard constraint on the tank water temperature to be within predefined limits, i.e. the user comfort reflected users' attitude for the violation of water temperature bounds. With increase of the tolerance degree, more violation of the tank water temperature from the comfort zone was allowed by the user.

An hybrid approach for WH scheduling under day-ahead variable prices that combined the model-free control based on reinforced learning and MPC was presented in [37]. The motivation behind such a hybrid model was that MPC techniques that could estimate parameters of the WH model (without explicit modeling) based on the historical data still required a user to connect domain knowledge to the experimental data, to decide the order and structure of the model. On the other hand, reinforced learning techniques are model free and do not require an expert knowledge, but require a big number of measurements. In the proposed hybrid approach, an MPC controller was used to learn and steer the WH during the first several days of the control period. After that, the knowledge acquired by MPC was utilized in a model-free Fitted Q-iteration (FQI) controller that relied on MPC as on an expert. The goal of both the MPC and FQI controllers was to minimize the heating costs resulting from the on/off action on the current time interval along with long term costs on all the coming intervals. The objective function additionally incorporated a weighted term responsible for relaxation of the user comfort constraints determined by the lower and upper tank water temperature boundaries suitable for the user.

A heuristic algorithm for scheduling of a single $\mathrm{WH}$ under variable electricity prices was presented by [55]. The algorithm simultaneously minimized the energy cost and potential user discomfort caused by the WH scheduling on a discrete timescale. More exactly, the objective function was to minimize a weighted sum of two normalized functions that represented the heating costs and user discomfort, respectively. The weighting factors used in the objective function served for balancing between the importance of satisfaction 
of two goals to the user. To solve the problem, a heuristic algorithm iterated through all the possible values of the user comfort weighting factor starting from the value equivalent to the worst user comfort allowed. At every iteration of the comfort weighting factor, the algorithm searched for the intervals where the user comfort violation was identified, switched on the $\mathrm{WH}$ in the preceding intervals and recalculated the tank temperature values while checking for safety temperature constraints violation, and (if necessary) updated the minimum found value of the objective function. The algorithm continued looping inside the current iteration of the weighting factor until there was no time slot where user comfort was compromised. The WH schedule obtained at the maximum value of the weighting factor at the last iteration corresponded to an optimal schedule that did not exceed the minimum energy cost by a predefined percentage value. At every time slot, the user comfort was modeled as a difference between the current and user-desired (fixed) temperature threshold multiplied by the mass of water delivered during that time slot.

A particle swarm optimization algorithm for a single WH under ToU tariff was presented in [38]. The goal was to minimize the electricity bill for water heating and maintain the customers hot water demand by rescheduling the operation time of the WH subjected to the expected temperature constraints. The Binary Particle Swarm Optimization (BPSO) algorithm for imitating animal populations' behavior (e.g., bird flocking) given some discrete locations was used for finding the best schedule of on/off states of the $\mathrm{WH}$ on a discrete timescale. Thus, the on/off states of the WH represented two possible locations of particles that had to chosen according the velocity, location and the fitness function value on every iteration of the BPSO algorithm subject to user comfort constraints for hot water. The user comfort was represented as a difference between the comfortable setpoint tank water temperature and the current temperature normalized in a range from 0 to 1 . The effect of the algorithm for reducing the costs of a single WH was also demonstrated on the aggregated WHs load consisting of multiple WHs. The authors claimed that the algorithm resulted in a flatter daily power demand curve thus improving the reliability of the grid segment.

An optimal scheduling algorithm for thermostatically controlled loads such as WHs operating under day-ahead real-time pricing (RTP) tariff was presented by [56]. The aim of the algorithm was to find the best tank water temperatures that minimize the energy costs and user discomfort. Additionally, the algorithm was capable to maintain the a time varying power bound under RTP. The scheduling problem was modeled as a weighted directed acyclic graph where each node represented a state of the WH (tank water temperature) and weights on the edges referred to the associated costs. More precisely, the weight of each edge was a value of a cost function comprising a weighted sum of both the normalized energy cost and the normalized comfort cost. The weight coefficients of the cost function signified the importance of energy cost reduction and comfort maintenance from the user perspective. A discrete time model was applied, meaning that the graph appeared as an assembly of layers representing different time slots that were activated when a control action was taken (temperature to be set at the current time slot). Noteworthy, the thermodynamic model accounted for the fact that users request the mixed tap rather than the water from the WH outlet. As such, the comfort model was based on the tap water temperature and tap water flow rate considerations. Specifically, the end-user had to provide the preferred tap water temperature, the minimum tolerable temperature and 
the tap flow rate during the hot water usage as input for the comfort model. The user input for the algorithm also included the acceptable deviation from the minimum energy cost. The Dijkstra's single-source shortest path algorithm was used for searching for the shortest path in the graph between the initial and final layer, i.e. such a path that had the smallest cumulative weight between the initial time slot and the final time slot. The algorithm iteratively adjusted the values of the weights of comfort and energy costs of the cost function, starting from initial values that get adjusted (decreased or increased) at each iteration until an optimum is reached.

A reinforced learning (RL) technique was applied by [57] for minimizing the energy consumption costs of a single WH. In the RL technique, the WH was considered as a blackbox whose essential properties are learned by an agent (controller) so that no modeling of the WH thermodynamics was required. The control of the WH was described by the Markov decision process where the current WH state stochastically depended on the state, the control action and the cost function at the previous discrete time step. The goal of the agent was to find the optimal on/off control actions for the $\mathrm{WH}$ at all the time steps in a planning period that minimize the heating costs and respect safety and user-defined tank water temperature constraints. A supervised learning algorithm was applied to train the neural network of the agent on all possible state-action pairs, resulting in a fitted state-action value function (Q-function). The obtained values of the fitted Q-function were further used to derive the probabilities of on/off control actions at every time interval considering the feature vectors.

Two control algorithms for scheduling of a single WH based on distinct thermodynamic models were proposed by [39]. The optimization objective was to minimize the heating costs based on day-ahead electricity spot market prices while fulfilling two constraints: (i) the water temperature inside the tank must always be below the maximum allowed temperature setting and (ii) the hot water temperature during hot water usage must exceed a defined minimum temperature. The optimization problem was formulated as a linear programming binary optimization problem and the subsequent presented optimal strategy was based on both the expected demand and a piecewise constant energy cost function. Two thermodynamic models were explored, namely the well-mixed model and multi-node model for which the optimization problem temperature constraints were applied only for the top node of the tank. The multi-node model of the WH led to an optimization problem with non-linear constraints due to the model's non-linear dependency from the hot water demand. An approximation approach based on estimates of the well-mixed model was applied to resolve such an optimization problem.

A control algorithm for scheduling multiple household loads comprising WHs under uncertain electricity prices was proposed by [58]. The optimal control algorithm utilized a discrete time model. The goal was to simultaneously minimize of electricity consumption costs, maximize the robustness of the heating schedule with respect to uncertain electricity price and to maximize the satisfaction of a consumer. Three objectives were employed in a single objective function. The consumption costs were formalized by using a predicted price variable that had some uncertainty due to the prediction errors. To reflect the effect of price uncertainty on energy consumption expense, the schedule robustness was introduced into the objective function. The robustness was represented by a variance of a random price variable. Consumer satisfaction with a WH schedule is formulated as unwillingness level of 
a consumer to perform WH load shifting assuming that the WH has a target load schedule fully comfortable to the user and that that schedule is given by a user. This unwillingness is formalized as a difference between the new and target power demand weighted with the coefficient of unwillingness. A convex optimization approach was applied to solve the problem.

An algorithm for scheduling thermostatically controlled household loads including WHs considering both payment and comfort was proposed by [59]. The objective of the optimization problem was to minimize the total electricity cost of the load schedule of the WH under the constraints that at any time the tank water temperature had to be in the comfort range set by consumers. The Traversal-and-Pruning algorithm was devised for solving that integer programming problem. The suggested algorithm was specific to the type of the controlled load and in the case of a WH it was based on a binary tree wherein each node represented the state of the $\mathrm{WH}$ and each layer corresponded for a new time step. The algorithm worked in several traversals of the tree. During the first traversal all the nodes were marked with computed tank water temperature values considering the time steps with hot water usage. The second traversal of the tree nodes was to identify which temperature values (or nodes) violated the user-defined temperature constraints while removing the subtrees that had them as a root node from the original tree. The final traversal was about to find which node in each layer was superior to his cousin nodes in terms of the lower costs and the same or better comfort and repeating this operation for the node's childs. For this procedure, the authors proposed a separate traversal algorithm claimed to produce the best water heating schedule in relatively short time.

An appliance commitment algorithm, which schedules thermostatically controlled appliances (TCAs), such as EWHs, was proposed by [60]. The objective was to minimize the electricity bill taking into consideration constraints set by comfort requirements. The two-step optimization framework proposed was capable to adjust the WH operation schedule to handle uncertainties caused by forecast errors in energy prices and hot water consumption. More specifically, at the first step the day-ahead prediction of prices and hot water usage was used to solve the deterministic optimal problem for the next day. At the second step, the schedule was re-optimized in real-time based on the updated information about the real-time market prices and hot water usage. This involved recalculation of the heat injections in the tank and times that WH had to spend in "on"-state in order to support the new hot water demand. Also, the estimated price curve was updated with respect to the real-time market prices. User comfort is represented by a set of linear box constraints that specify the desired tank water temperature values at every time interval.

A system that helps residential consumers to respond to variable electricity prices was presented in [61]. The underlying multi-objective control algorithm of the system aimed at minimizing the electricity costs for water heating and hypothetical user inconvenience that originated from modifying the normal operation of a WH. As input the system used load profiles containing residents' preferred times of hot water demand and average power consumption levels of the WH. The WH was scheduled with some given tolerance near the time-slots of a regular hot water usage. The user discomfort was modeled as a distance between the new startup time and the one in the preferred usage profile. However, if the load fell inside the interval of regular usage, then inconvenience accounted only for the power difference (e.g., modulating WHs). 
A control strategy that aimed at maximizing the net monetary benefit derived from energy services provided to a user by home devices comprising a $\mathrm{WH}$ was presented in $[62,63]$. A binary particle swarm optimization approach was used for searching the optimized on/off schedule for the WH. Interestingly, the user comfort was considered from two perspectives. The first one was related to the thermal energy content of water delivered to and experienced by a user. The other aspect described the time varying benefit (importance) of that energy for an individual over time and was modeled as the cost of undelivered service (the amount of hot water that was not delivered) translated into a virtual price $[\$ / \mathrm{kWh}]$.

In [64], a load control strategy as a user-centered supporting tool for balancing residential consumers' comfort and price preferences was proposed. The objective was to simultaneously satisfy two conflicting goals, i.e., minimizing the total energy costs for water heating and minimizing the deviation of hot water temperature of the WH's outlet from the desired setpoint. The proposed control strategy operated each $\mathrm{WH}$ at its maximum rate during low-cost periods and lowered the power requirements (through thermostat settings) during the high-price periods, while keeping the temperature of the outlet hot water within user comfortable bounds.

A model-predictive control (MPC) strategy to derive optimal switching times of a single WH under dynamic pricing was proposed by [65]. The MPC algorithm aimed at simultaneous minimization of the heating costs and user feeling of discomfort while taking into account thermal dynamics of the WH. The objective function of MPC was formulated as a weighted sum of two terms that respected the goals of the total costs reduction and the user discomfort minimization arising from the WH scheduling. More specifically, the heating costs were considered to be the total cumulative heating costs computed from the start of the MPC algorithm. Whereas the user comfort was modeled by a squared cumulative difference between the current tank water temperature and user-defined comfortable temperature, making the problem a nonlinear least-squares optimization problem.

A setpoint control strategy to shift the power demand of multiple WHs to the low-price periods was proposed by [66]. The goal was both to reduce the peak load and electricity costs by shifting the $\mathrm{WH}$ loads outside the high price periods. The proposed control algorithm utilized the idea of water pre-heating similar to [67]. The water pre-heating was initiated prior to the intervals of high prices by raising the thermostat setpoint temperature above normal and thus initiating an extra reserve of the thermal energy in the WH tank. Further, the heating elements were switched off during the period of high prices unless the tank water temperature droped below the lower thermostat threshold. The extra heat reserve and the capability to activate the water heating on violation of the lower thermostat threshold supported user comfort during the WH disconnection period. After the highprice period the heating elements were turned on to recover the water temperature back to the upper thermostat setpoint as in the conventional deadband control.

Several strategies for peak load reduction under variable pricing in application to WHs were examined by [68]. In the first strategy, the timed power interruption at specific times was implemented similar to DLC. The second strategy was to control the upper and lower thermostat setpoints by a two-period timer setback thermostat with selectable periods and selectable change to the lower limit (the margin between two setpoints was a fixed offset $5^{\circ} \mathrm{C}$ ). The last strategy simulated a price-sensitive thermostat that controlled the 
lower setpoint temperature in response to the price of electricity. More specifically, in this strategy, given the range of daily price variation the controller adjusted the lower limit so that it reached its minimum value when the price is at a maximum. The price-sensitive thermostat strategy found to be the most advantageous of three strategies in terms of user comfort and consumer savings. However, that strategy did not consider user comfort and thus did not always guarantee a user comfortable tank water temperature.

\subsubsection{SUMMARY}

In the following, we first summarize essential features of the incentive-based and pricebased solutions found in the literature in Table 2.1 and Table 2.2, respectively. Next, we briefly discuss significant aspects of the reviewed solutions.

As one can conclude from two tables, the number of deterministic algorithms overwhelms the number of stochastic, artificial intelligent (AI) and engineering-based solutions. This can be explained by difficulties with obtaining the probability distributions or fuzzy membership functions [36], big amount of data required for reinforcement learning (RL) [37] and big computational costs of stochastic solutions [60]. Although most of the solutions are heavily affected by an unexpected variation of input variables (e.g., an unforseen intense hot water demand), some of them yet specifically consider such uncertainties [34, 36, 58, 60].

As one can notice, there is a vast variety of optimization approaches inherent the incentive-based and price-based solutions. As contrasted, more engineering solutions [33, $47,51]$ are usually driven by iterative algorithms to adjust controlled signals based on a simple logic. In the reviewed literature, incentive-based approaches pursue different utility-oriented goals like peak load reduction [20, 43, 46], support of frequency [33] and voltage [32] regulation or combination of them [44], whereas the price-based solutions are mainly focused on reduction of a consumer electricity bill [34-36]. A couple of strategies use WH load shifting outside high-price periods to diminish stress on the grid $[66,68]$.

Because of their utility-oriented nature most of the incentive-based solutions are coordinated in a centralized way by energy companies [20, 32, 33]. On the contrary, solutions implemented in a decentralized way on the consumer side [48-50] are usually consumer-driven and are part of home energy management systems that control various domestic appliances. On the other side, most of the price-based solutions are decentralized and, as mentioned earlier, they assists the consumers in cutting their electricity bills. Implemented in a centralized way some incentive-based solutions deal with aggregated load models of WHs [41] (rather than modeling a single WH) and perform control over clusters of WHs [21, 32, 41, 47].

Alteration of the conventional deadband control can lead to the lack of hot water in the WH tank and discomfort to occupants. That is why the topic of the end-user satisfaction takes a special place in the reviewed references. In a substantial number of papers, the user comfort is modeled as a deviation of the tank water temperature from predefined thresholds [37-39, 41, 44, 64]. Such thresholds can change over the time [20, $50,52,57,59,60]$ or vary due to a time-dependent weight factor [20,69]. Noticeably, apart from approaches that describe user discomfort only as a mismatch between instantaneous values of the water temperature and predefined threshold(-s) [49, 51], other approaches capture the temperature deviations together with duration of time intervals in which they occur $[48,53,61]$. 
Table 2.1: Features of state-of-the-art incentive-based control mechanisms for WHs.

\begin{tabular}{|c|c|c|c|c|c|c|c|c|c|c|c|c|c|c|c|}
\hline & {$[20]$} & [32] & [33] & [43] & [46] & [44] & [41] & {$[47]$} & [48] & [49] & {$[50]$} & [51] & {$[52]$} & [21] & [53] \\
\hline $\begin{array}{c}\text { Control type: } \\
\text { deterministic }(\delta) \text {, } \\
\text { stochastic }(\operatorname{Pr}), \\
\text { engineering }(\circledast), \\
\text { other }(\psi)\end{array}$ & $\delta$ & $\delta$ & 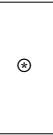 & $\delta$ & $\psi$ & $\delta$ & $\delta$ & $\delta$ & $\delta$ & $\delta$ & $\delta$ & $\circledast$ & $\delta$ & $\psi$ & $\delta$ \\
\hline $\begin{array}{l}\text { Optimization approach: } \\
\text { linear } \\
\text { programming(LP), } \\
\text { dynamic } \\
\text { programming(DP), } \\
\text { integer linear } \\
\text { programming (ILP), } \\
\text { reinforcement } \\
\text { learning(RL), } \\
\text { heuristics(H), } \\
\text { rule-based(R), none }(\boldsymbol{X})\end{array}$ & DP & LP & $x$ & $\mathrm{H}$ & $\mathrm{RL}$ & $\mathrm{R}$ & LP & $x$ & $\mathrm{R}$ & ILP & LP & $x$ & LP & other $^{\mathrm{a}}$ & $\mathrm{H}$ \\
\hline $\begin{array}{c}\text { Objective: peak load } \\
\text { reduction }(\checkmark) \text {, voltage }( \\
\varpi) \text { or frequency }(\mho) \\
\text { regulation }\end{array}$ & $\checkmark$ & $\checkmark, \infty$ & $\sigma$ & $\checkmark$ & $\checkmark$ & $\checkmark, \infty$ & $\checkmark$ & $\checkmark$ & $\checkmark$ & $\checkmark$ & $\checkmark$ & $\checkmark$ & $\checkmark$ & $\checkmark$ & $\checkmark$ \\
\hline Control of a single $\mathrm{WH}$ & 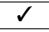 & $x$ & $\checkmark$ & $\checkmark$ & $\checkmark$ & $\checkmark$ & $x$ & $x$ & $\checkmark$ & $\checkmark$ & $\checkmark$ & $\checkmark$ & $\checkmark$ & $x$ & $x$ \\
\hline $\begin{array}{c}\text { Electricity consumption } \\
\text { optimized }\end{array}$ & $\checkmark$ & $x$ & $x$ & $\checkmark$ & $\checkmark$ & $x$ & $x$ & $x$ & $x$ & $x$ & $x$ & $x$ & $\checkmark$ & $x$ & $x$ \\
\hline $\begin{array}{c}\text { User comfort in } \\
\text { objective function }(\boldsymbol{V}) \text {, } \\
\text { constraints(), other(§), } \\
\text { none }(\boldsymbol{X})\end{array}$ & $\checkmark$ & & $\S^{(\mathrm{b})}$ & $\checkmark$ & $\S^{(\mathrm{c})}$ & & & $x$ & $\S^{(\mathrm{d})}$ & & $\checkmark$ & $\S^{(\mathrm{b})}$ & $\checkmark$ & $\S^{\text {(c) }}$ & $\checkmark$ \\
\hline $\begin{array}{c}\text { Comfort model based } \\
\text { on tank water } \\
\text { temperature }(\checkmark) \text {, tap } \\
\text { water(), other(§), } \\
\text { none }(\boldsymbol{X})\end{array}$ & $\checkmark$ & $\S^{(\mathrm{g})}$ & $x$ & $\checkmark$ & $\boldsymbol{J}^{(\mathrm{e})}$ & $x$ & $\checkmark$ & $x$ & $\S^{(\mathrm{f})}$ & $\S^{(\mathrm{g})}$ & $\checkmark$ & $x$ & $\checkmark$ & $\S^{(\mathrm{h})}$ & $\checkmark$ \\
\hline $\begin{array}{c}\text { Time-varying comfort } \\
\text { requirements }\end{array}$ & $\checkmark$ & $\checkmark$ & $x$ & $x$ & $x$ & $x$ & $x$ & $x$ & $\checkmark$ & $\checkmark$ & $\checkmark$ & $x$ & $\checkmark$ & $\checkmark$ & $\checkmark$ \\
\hline $\begin{array}{c}\text { WH thermal dynamic } \\
\text { model }\end{array}$ & $\checkmark$ & $x$ & $x$ & $\checkmark$ & $\checkmark$ & $\checkmark$ & $\boldsymbol{x}^{(\mathrm{i})}$ & $\checkmark$ & $x$ & $x$ & $x$ & $\checkmark$ & $\checkmark$ & $\checkmark$ & $x$ \\
\hline $\begin{array}{c}\text { WH tank model: } \\
\text { well-mixed }(\mathcal{V}), \\
\text { multi-node }(), \operatorname{none}(\boldsymbol{X})\end{array}$ & & $x$ & $x$ & $\checkmark$ & $\checkmark$ & $\checkmark$ & $x$ & & $x$ & $x$ & $x$ & & $\checkmark$ & $\checkmark$ & $x$ \\
\hline $\begin{array}{c}\text { On/off control of } \\
\text { heating elements }(\checkmark), \\
\text { setpoint temperature } \\
\text { control }\left({ }^{\circ} \mathrm{C}\right) \text {, other }(\S)\end{array}$ & $\checkmark$ & $\S^{(j)}$ & $\S^{(\mathrm{k})}$ & $\checkmark$ & $\checkmark$ & ${ }^{\circ} \mathrm{C}$ & $\checkmark$ & $\checkmark$ & $\checkmark$ & $\checkmark$ & ${ }^{\circ} \mathrm{C}$ & $\checkmark$ & $\checkmark$ & ${ }^{\circ} \mathrm{C}$ & $\checkmark$ \\
\hline
\end{tabular}

(a) Solution based on fuzzy logic approach.

(b) User comfort is ensured by letting the upper thermostat of a dual-element WH to operate as usual.

(c) Fuzzy membership function(s).

(d) Comfort metric used only for evaluation.

(e) Besides temperature, the comfort also accounted for the hot water outflow from the $\mathrm{WH}$

(f) Comfort was based on the time delay of comfortable water temperature.

(g) Comfort was based on the power demand constraints.

(h) Comfort was based on historical WH power demand profiles and the average lower and upper thermostat setpoints of population of WHs.

(i) Thermodynamics of an aggregated WH load was modeled via histograms containing the random temperature distribution.

(j) Voltage control of WHs was implemented.

(k) Temperature-based control is coupled with frequency control.

For the solutions that utilize predefined (sometimes fixed) temperature threshold the tank water remains hot even during the periods of no hot water usage [37, 39, 43, 64]. High tank water temperatures at times when no hot water is needed result in unwanted heat losses and consequently extra electricity consumption. Although several solutions allow for minimization of the electricity consumption $[20,43,46]$ next to the claimed reduction of the peak load, the minimization of the stand-by heat losses is not considered as optimization goal. Despite most of the solutions that incorporate thermal dynamic models of the $\mathrm{WH}$ 
Table 2.2: Features of state-of-the-art price-based control mechanisms for WHs.

\begin{tabular}{|c|c|c|c|c|c|c|c|c|c|c|c|c|c|c|c|c|c|c|}
\hline & [34] & [35] & {$[36]$} & [37] & [55] & [38] & {$[56]$} & {$[57]$} & [39] & {$[58]$} & [59] & {$[60]$} & $\begin{array}{l}61] \\
\end{array}$ & [63] & [64] & [65] & {$[66]$} & [68] \\
\hline $\begin{array}{c}\text { Control type: } \\
\text { deterministic }(\delta), \\
\text { stochastic }(\operatorname{Pr}) \\
\text { other }(\psi)\end{array}$ & $\delta$ & $\delta$ & $\operatorname{Pr}$ & $\psi$ & $\delta$ & $\delta$ & $\delta$ & $\psi$ & $\delta$ & $\operatorname{Pr}$ & $\delta$ & $\delta$ & $\delta$ & $\delta$ & $\delta$ & $\delta$ & $\delta$ & $\delta$ \\
\hline $\begin{array}{c}\text { Optimization } \\
\text { approach: mixed } \\
\text { integer (non)linear } \\
\text { programming } \\
\text { (MI(N)LP) linear } \\
\text { programming } \\
\text { relaxation(LPR), } \\
\text { none }(\boldsymbol{X})^{\dagger}\end{array}$ & LP & MILP & $\mathrm{H}$ & RL & $\mathrm{H}$ & $\mathrm{H}$ & other & RL & LP & LPR & MINLP & LP & LP & LP & LP & $\mathrm{H}$ & $x$ & $x$ \\
\hline $\begin{array}{c}\text { User comfort in } \\
\text { objective } \\
\text { function }(\checkmark) \text {, } \\
\text { constraints() or } \\
\text { none }(\boldsymbol{X})\end{array}$ & $\checkmark$ & $\checkmark$ & $\checkmark$ & & $\checkmark$ & & $\checkmark$ & & & $\checkmark$ & & & $\checkmark$ & $\checkmark$ & $\checkmark$ & $\checkmark$ & $x$ & $x$ \\
\hline $\begin{array}{c}\text { Comfort model } \\
\text { based on tank } \\
\text { water } \\
\text { temperature }(\boldsymbol{V}) \text {, } \\
\text { tap water }(\text {, } \\
\text { other }(\S), \operatorname{none}(\boldsymbol{X})\end{array}$ & $x$ & & $\checkmark$ & $\checkmark$ & (a) & $\checkmark$ & (a) & $\checkmark$ & $\checkmark$ & $\S^{(b)}$ & $\checkmark$ & $\checkmark$ & $\S^{(c)}$ & $\S^{(\mathrm{d})}$ & $\checkmark$ & $\checkmark$ & $x$ & $x$ \\
\hline $\begin{array}{l}\text { Time-varying } \\
\text { comfort } \\
\text { requirements }\end{array}$ & $\checkmark$ & $\checkmark$ & $\checkmark$ & $x$ & $\checkmark$ & $x$ & $\checkmark$ & $\checkmark$ & $x$ & $\checkmark$ & $\checkmark$ & $\checkmark$ & $\checkmark$ & $\checkmark$ & $x$ & $\checkmark$ & $x$ & $x$ \\
\hline $\begin{array}{l}\text { WH thermal } \\
\text { dynamic model }\end{array}$ & $\mathrm{n} / \mathrm{a}^{(e)}$ & $\checkmark$ & $\checkmark$ & $\checkmark$ & $\checkmark$ & $\checkmark$ & $\checkmark$ & $\mathrm{n} / \mathrm{a}^{(\mathrm{f})}$ & $\checkmark$ & $x$ & $\checkmark$ & $\checkmark$ & $x$ & $\checkmark$ & $\checkmark$ & $\checkmark$ & $x$ & $x$ \\
\hline $\begin{array}{c}\text { WH tank model: } \\
\text { well-mixed }(\checkmark), \\
\text { multi-node }(), \\
\text { other( }(\S) \text {, none }(\boldsymbol{X})\end{array}$ & $x$ & $\checkmark$ & $\checkmark$ & $\checkmark$ & $\checkmark$ & $\checkmark$ & $\checkmark$ & $x$ & $\boldsymbol{J}^{(\mathrm{g})}$ & $x$ & $\checkmark$ & $\checkmark$ & $x$ & $\S \mathrm{h})$ & $\S^{(i)}$ & $\checkmark$ & $x$ & $x$ \\
\hline $\begin{array}{c}\text { On/off control of } \\
\text { heating } \\
\text { elements }(\checkmark) \text {, } \\
\text { setpoint } \\
\text { temperature } \\
\text { control }\left({ }^{\circ} \mathrm{C}\right) \text {, } \\
\text { other }(\S)\end{array}$ & $\checkmark$ & $\checkmark$ & $\checkmark$ & $\checkmark$ & $\checkmark$ & $\checkmark$ & $\checkmark,{ }^{\circ} \mathrm{C}$ & $\checkmark$ & $\checkmark$ & $\checkmark$ & $\checkmark$ & $\checkmark$ & $\checkmark$ & $\checkmark$ & $\checkmark$ & $\checkmark$ & ${ }^{\circ} \mathrm{C}$ & ${ }^{\circ} \mathrm{C}$ \\
\hline $\begin{array}{c}\text { Uncertainties } \\
\text { handled: price }(€) \text {, } \\
\text { hot water } \\
\text { demand }(), \\
\text { comfort }(\boldsymbol{X}) \text { or } \\
\text { none }(\boldsymbol{X})\end{array}$ & & $x$ & $€, \otimes$ & $x$ & $x$ & $x$ & $x$ & $x$ & $x$ & $€$ & $x$ & $€$ & $x$ & $x$ & $x$ & $x$ & $x$ & $x$ \\
\hline
\end{tabular}

(†) The rest of the categories are as in Table 2.1

(a) Comfort model also accounted for the tap water flow rate.

(b) Comfort was modeled through the load profile time offsets.

(c) Comfort was represented via the load profile time offsets and power differences.

(d) Comfort defined by thermal energy of delivered water and importance of the hot water service to the user

(e) The approach was specifically tailored to deal with the absent tank water temperature measurements.

(f) A model-free approach was applied in which the properties of the WH were learned through the tank water temperature measurements.

(g) The presented control is also demonstrated based on multi-node model of the WH tank.

(h) Two-mass composite model based on tracking the height of the upper hot water section.

(i) A simplified model based on the WH energy factor, water outflow rate and difference between the inlet and outlet water temperatures was employed.

account for the heat losses, heat losses are considered only through their impact on the tank water temperature and are not represented as a separate objective function.

A more stochastic approach for comfort representation in [36] considers user comfort as a degree of user tolerance to the tank water temperature violations of the comfort zone. The comfort is represented by three time-based parameters that signify how much the user is willing to reduce his comfort in [34]. A control approach that allows for comfort constraints relaxation was proposed by [37]. It refers two the fact that sometimes users might opt in favor of money savings and tolerate lower temperatures.

To describe user comfort, $[62,63]$ use a heuristic metric that treats the user comfort in terms of importance of hot water to the user during a day and the thermal energy of hot 
water discharged from the WH. In [65], the user (dis)comfort is expressed in the form of a cumulative difference between the desired and the current tank water temperature taken over time, which might point to the need to consider accumulation of user inconvenience in time. Other non-temperature comfort modeling approaches are based on the allowed time of use of the WH [48], the time distance of the new WH load schedule from user comfortable times [61], the time during which the tank water temperature is beyond the discomfort threshold [53], power demand constraints [49], user unwillingness to shift the WH load [58], or comfort is ensured by engineering specifics of operation [51].

A few solutions highlight the fact that residents seldom use hot water directly from the WH tank $[35,55]$ and account for the presence of the tap mixers in home environments. The comfort is represented there by an absolute difference between the mixed tap water temperature and the desired one $[35,55]$, sometimes also combined with the tap water flow rate so that the user comfort appears as the thermal energy delivered to the user $[55,56]$.

A common practice to consider the user comfort is by introducing additional constraints into the optimization problem such as minimization of load during the peak demand periods or minimization of the heating costs. These constraints restrict the decision variables (e.g., setpoint temperature) of the problem in order to provide the tank water temperature above the minimum value or within predefined limits [37-39]. Other papers embed the user comfort metric directly in the objective function and solve a multi-objective optimization problem [34-36]. This is typically done by adding a comfort-related term into the existing objective function $[34,55,58]$. Minimization of the user discomfort (or maximization of comfort) as a standalone objective function minimization can be found in $[50,53,64]$. Interestingly, the problem of the peak load reduction is approached by minimizing the reduced user comfort subject to the power demand constraints in [50]. By representing the user comfort in the objective function, feasible solutions are allowed to provide water temperatures also below the predefined threshold, leading to increased flexibility compared to hard constraints [55]. Attempts to introduce tolerance degree for comfort constraint violations were made in [36, 37].

Among the solutions that model the WH thermal dynamics, a dominating number utilizes a well-mixed WH tank model explaining it with sufficient accuracy of well-mixed models, the computational complexities and the need to specify a greater number of parameters when dealing with the WH stratification $[43,55]$.

Considering the implementation of the WH control, the most popular approach is to operate the state of WH heating elements by directly switching them on/off [20, 43, 46], while control of the WH thermostat setpoints seems to be used less frequently [44, 50].

As a summary of our observations, we can highlight the following aspects relevant for the current work:

- most of the aforementioned solutions use the water temperature at the outlet of the WH to describe user comfort. However, residents usually perceive the mixed tap water during hot water activities (WAs) which makes (i) the mixed tap water temperature and (ii) personal comfort zone justified candidates to consider in the user comfort model.

- user comfort is usually quantified by the difference between the current temperature and the predefined threshold(s), which refers to only an instantaneous discomfort 
value. On the other hand, user inconvenience can change over time and depend on the previous values during the same WA. For example, the user can tolerate water temperature drop of $5^{\circ} \mathrm{C}$ if it lasts no longer than 10 seconds. Here besides the temperature constraint we need to keep track of the time during which the colder water is supplied.

- some references point to a possible user tolerance to colder temperatures during hot water usage and account for it in control [34, 36];

- as a rule, minimization of the heat losses is not formulated as a separate optimization goal, which leads to unnecessary stand-by heat losses and parasitic electricity consumption.

- the above solutions control the tank water temperature variable, although the usercomfortable tap water temperature also depends on the hot water flow from the WH.

\subsection{Necessity of Conside Ring Heat Losses}

An impressive number of approaches reviewed in Section 2.2 ensures user comfort by requiring the tank water temperature to be always above a predefined setpoint that "represents" user comfort. Usually, the lower setpoint temperature is chosen in the range $50-60^{\circ} \mathrm{C}[37-39]$. As a result, the tank water is often above the room temperature (e.g., $24^{\circ} \mathrm{C}$ ) when no hot water is needed $[20,37]$. In case the time lag between two WAs is relatively big, the stored heat will be partly dissipated to the environment and tank water will be heated again to compensate the wasted heat. On the contrary, it would be more energy efficient to heat the water right upfront the WA so that the heat waste is minimized. Furthermore, different WAs usually require distinct amounts of heat, meaning that the heat unutilized during the WAs contributes to the heat losses.

Although several DR solutions found in the literature deal with minimization of the electricity consumption during peak demand periods [20, 43, 46, 70], which should lead to smaller operational temperatures and reduced heat losses during such periods, they do not explicitly minimize the heat losses during a day. For example, the peak load reduction problem solved by [20] was formulated as minimization of the total number of intervals where the $\mathrm{WH}$ was switched on during the day and night peak demand periods. The heat losses are not minimized outside these control intervals which results in the tank water temperature values above $55^{\circ} \mathrm{C}$ and undue heat losses.

On the other side, reduction of heat losses is tightly connected with the user comfort, because zero heat losses would mean close to the cold water temperature for the user. Therefore, the task of minimization of the heat losses should go hand in hand with minimization of user comfort disruptions.

\subsection{Necessity of Conside Ring USE R COMFORT}

When modifying the deadband control according to the goals of a utility company (e.g., peak load reduction) or consumer (e.g., electricity bill), the resulting changes in the water heating profile may lead to lower tank water temperatures than those required by users. 
Since consumers put equal value on money savings, environmental footprints and their personal comfort [8,29-31], the increasing dissatisfaction with water heating can outweigh the desire to fulfill other objectives so that the consumer may reject the benefits of the incentive-based and(or) price-based DR.

Therefore, it is important to know the boundaries of user comfort and to what extent the users can tolerate temperature deviations in order to (1) perform control with a full customer satisfaction and (2) implement the control efficiently on the edge of user comfort so that the electricity is consumed at the the minimum yet acceptable to the user comfort level [36,37]. This section presents essential aspects of user comfort modeling during hot water usage.

\subsubsection{Thermal Comfort Modeling}

While user comfort is a well investigated topic for space heating loads and is a subject of international standards [71, 72], the topic of modeling the user comfort during the hot water usage is less mature. Unlike the space heating, an individual is in a direct body-contact with water when performing hot water activities (e.g., washing hands, showering) rather than protected by clothing, i.e. people experience and appreciate the physical properties of water directly with their bodies.

Some attempts have been made to account for user thermal comfort during control of WHs [20, 32, 34, 35]. Based on the variables used for user comfort representation, these attempts can be divided into those that model user comfort via the tank water temperature $[20,37,38]$ and via the tap water temperature $[35,55]$. The user comfort is often described by an instantaneous deviation of the current water temperature from predefined threshold(s). Most of the existing approaches that account for the mixer tap have the following limitations:

- no representation of the personal feeling of water temperature;

- the user ability to tolerate colder temperatures is not present;

- user comfort is an instantaneous value (i.e., discomfort cannot depend on previous values).

Thermal comfort can be defined as that condition of mind which expresses satisfaction with the thermal environment and is assessed by subjective evaluation [73]. From this definition one can conclude that the thermal comfort is based on the state of mind and "subjective evaluation", meaning that comfort is a cognitive process that involves many inputs including physical, physiological, psychological, and other processes. Being a result of subjective evaluation, the user comfort model requires some additional parameters that can describe its subjective nature.

The feeling of temperature is tightly connected with thermoregulation processes in a human body. The thermal physiotherapy considerations below support the ideas of the possible user comfort tolerance and importance of duration of the user comfort violations.

It has been found that individuals can draw conclusions about personal thermal comfort directly from temperature sensations of the skin, deep body temperature as well as efforts to regulate body temperatures [73]. Thermal sensation can be defined as how people "feel" temperature of the surrounding environment (warm, neutral, cold, etc.) through their 


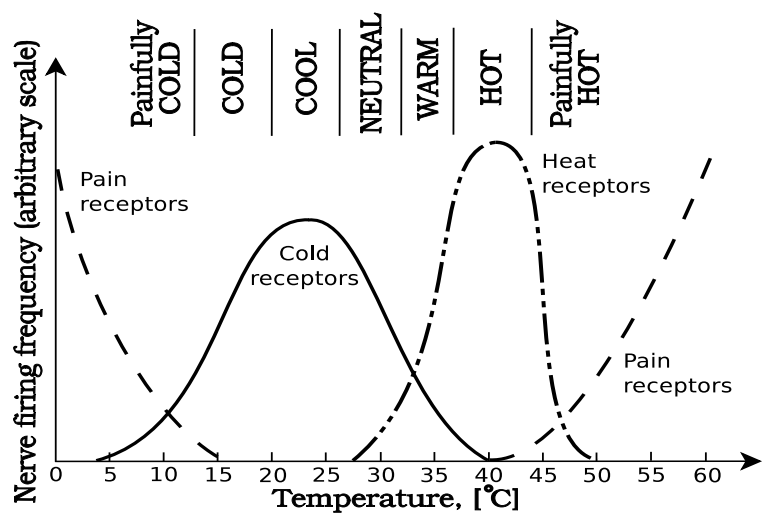

Figure 2.2: Types of temperature receptors in a body (adapted from [75]).

nerve endings [74]. Factors such as the area of the body, body position, duration of the stimulus on the body, initial thermal (humidity) state, temperature intensity and rate of change influence the thermal sensation.

Thermal physiotherapy suggests two types of receptors in a human body which give rise to the sensations of cold, hot and pain [75]. By sending electrical pulses (action potentials) to the hypothalamus with a certain frequency and amplitude, the heat receptors and cold receptors inform the brain about the need for thermoregulation of the body. These receptors are distinct by nerve firing frequencies and by the temperature ranges in which they are activated as illustrated in Figure 2.2.

The higher is the nerve firing frequency $(\mathrm{Hz})$, the better a human can distinguish that temperature from other temperatures. As it can be seen from Figure 2.2, the cold receptors are most active in the wider temperature range $20-27^{\circ} \mathrm{C}$. The heat receptors have the highest nerve frequency in the narrow range of temperatures $37-42^{\circ} \mathrm{C}$. This means that people sense the environment as comfortably cold (meaning that they do not distinguish temperatures as different) in the wider range of temperatures than for hot water. In other words, people are more sensitive to hot water temperature changes than to changes of cold water. This also refers to the ability of users to tolerate tap water temperature deviations.

\subsection{Modeling Water Heater Operation}

Different approaches for WH scheduling model the operation of the WH with different detailization as outlined in Section 2.2. Besides various engineering configurations of WHs, models account for physical phenomena that take place inside a hot water tank. Among the solutions that account for thermal dynamic processes inside the WH tank, the most popular models are the well-mixed (single-node) $[35,36,44,46]$ and multi-node $[20,47,51]$ models of the WH tank. Less popular models include two-mass composite (2MC) [63, 76] and thermocline tacking (TT) models [77, 78].

Both the well-mixed and multi-node models are based on the principle of the balance of energy flows in the WH tank. The only major difference between the a well-mixed and multi-node model is that the second type accounts for the natural convection causing 
stratification of water layers with different densities. There are arguments for and against both the well-mixed and multi-node models.

Advocates of the multi-node models and tank stratification argue that the well-mixed model no longer accurately models the temperature distribution in the $\mathrm{WH}$ after a WA has occurred, which will result in an underestimation of the outlet temperature, and consequently of the energy lost during usage events [77].

On the other side, supporters of the well-mixed models claim that multi-node models are sophisticated and that sufficient accuracy can be achieved by means of well-mixed models that have much simplier implementation considering the number of equations and the need to specify a greater number of parameters $[43,55]$.

We further provide our own analysis of pluses and minuses of stratified and nonstratified models. The technical parameters of the adopted WH model are presented in Appendix I.

\subsubsection{STRATIFIED AND WELl-MiXed TANKS}

One of the phenomena that requires special attention is stratification of water inside a $\mathrm{WH}$ tank. It has a direct effect on energy efficiency of a WH, and thus it is important for consideration. Stratification takes place when layers of water inside the tank become thermally distinguishable due to a higher density of cold water (free convection).

Stratification is a natural process that takes place even if all the water inside the tank is initially at uniform temperature. This happens because the water close to the tank walls has a lower temperature than the water at the center (temperature gradient). The relative cooler water flows down along the tank wall while the hotter water rises up in the center of the tank, forming separate thermal layers separated by a thermolcline. In this regard, heat losses is a natural enabler stratification [79]. There are multiple factors affecting this phenomenon. Factors such as insulation, ambient temperature, arrangement of heating elements, sizes, the shape and orientation of a tank, natural convection (varied water densities), forced convection of an external hydraulic system (e.g., pump), thermal conductance between the water layers, turbulence and heat diffusion inside the tank are amongst them.

Ideal stratification presupposes ideally isolated thermal layers in the tank. Cold water layer then 'pushes' all the hot water at the top without no mixing. Such idealistic operation allows to deliver water at its hottest to the tank exit pipe, i.e. without 'wasting' its heat content on the way. Which means that hot water is either available instantaneously and at its maximum temperature, or not available at all in case all hot water pushed out from the tank. As such, the volume of water that has to be heated again during the heat recovery after the water event is limited to the volume of hot water taken. While in the case of a well-mixed tank all the water has to be heated. By that means, stratification improves energy efficiency of a WH.

Importantly, the stratification is getting lost when the turnover of water in the tank increases. When the outlet valve of a fully charged WH is open, the hot water at the outlet has the maximum temperature that remains stable for some period of time. While the increasing layer of cold water at the bottom 'pushes' out hot water from the top, a layer of mixed water in between these two layers grows. The latter region causes a steady drop of temperature after some time and stratification breaks. 
Stratification is advantageous because the temperature at the top of the tank is higher than in a comparable well-mixed tank without stratification ${ }^{2}$, meaning that the comfort temperature is more often available for user in a stratified tank. This also means that less outflow of hot water is needed from a stratified tank in order to supply a comfortable temperature as compared to the well-mixed tank. Which means that a smaller inflow of cold water into the tank (equal to outflow of hot water) induces less disturbance of the thermal layers (forced convection) and further facilitates the stratification.

Despite the mentioned advantages of stratification, we utilize a well-mixed tank model in our studies as a simplified and extreme case of broken stratification. Unlike the stratified models, the well-mixed WH model treats the water temperature inside the tank as uniform. In this way, the well-mixed model refers to an extreme scenario where no stratification is present. The choice of the well-mixed model can be justified by:

1. a temporary effect of stratification in case of intense hot water drains and(or) frequent WAs common for households;

2. the proposed control strategy aims at minimizing the heat losses and thereby the natural convection that causes stratification;

3. simplicity of modeling the processes of charging water with heat and discharging of the accumulated heat from the tank;

4. simplicity of computing the heat losses, meaning that it is needless to consider the heat losses of stratified water layers separately.

Firstly, the stratification exists only for a limited time from the start of a hot water activity, because the cold water entering the tank fuses with hot water and breaks the thermocline that separates the hot and cold water layers. The loss of stratification increases with increase of the hot water flow rate. While for big storages (e.g., 200 [L]) the stratification may last up to 8.5 and 25 [min] under the initial tank water temperature at $60^{\circ} \mathrm{C}$ and 10 and $5[\mathrm{~L} / \mathrm{min}]$ water flow rates, respectively[80]. Small WHs demonstrate that stratification destroys already after 2.5 and 7.5 [min] under the same initial tank temperature and flow rates. A rough estimation based on the measurements obtained by [80] shows that stratification is present only $20 \%$ of the time of a 12.5 -minute WA under $10[\mathrm{~L} / \mathrm{min}]$ hot water flow rate (50-liter WH). Many experimental and numerical studies of thermal stratification in water storage tanks indicate that stratification degrades faster for smaller tank volumes under the same conditions [80-84]. In addition, if WAs occur close to each in time the stratification can be dissolved by the preceding WAs.

Secondly, one of our goals is to reduce the energy consumption of the WH by minimizing the heat losses. To achieve this goal, we intend to keep the tank water temperature as close as possible to the room temperature during the periods of no hot water usage, while the water is heated only prior the hot water usage. As a result, most of the time the $\mathrm{WH}$ operates at lower water temperatures than during the deadband control. Consequently, the water gets cold at the bottom of the WH tank significantly slower which impedes stratification.

\footnotetext{
${ }^{2}$ Average temperatures are the same in case of the same amount of energy stored
} 
Also, because every time the water is heated in conformity with the user comfort needs for the upcoming WA, the heat created in the tank is almost fully utilized during that WA in our approach (unless the water is heated at the same time for more than one WA). Additionally, we control the values of hot water flow rate during WAs so that the tank water is maximally utilized during the WA. This means that we do not contribute to stratification between WAs, but only during some time from the start of a WA when not all the hot water has left the tank yet.

As it will be shown in the next section, the complexity of well-mixed models boils down to a single first order differential equation that governs the tank water temperature. On the other hand, a multi-node model requires a separate equation for every stratified layer.

Last but not the least, computation of the heat losses is significantly simpler in the well-mixed model. This is because we do not need to consider multiple layers each having different temperature as in the multi-node model. In general, a well-mixed model is considered as a sufficiently accurate model for residential WHs [85].

The downside of using a well-mixed model is that there is a need to heat all the water in the tank in order to achieve the wanted tank water temperature, as opposed to the stratified tanks where the hot water is concentrated mainly in the upper part. As a consequence, a WH with a well-mixed tank is expected to consume more energy for water heating as compared to the same WH with a stratified tank.

\subsubsection{Utilized Water He ater Model}

We employ a well-mixed model of the WH suggested by [85]. In this model, the water temperature is uniformly distributed inside the WH tank. Similar to [55], we assume that all the water is heated by a single heating element located at the bottom of the tank. Let us consider the model in more detail. In a general form, the model expresses the energy conservation law of the WH tank:

$$
M C_{p} \frac{d T}{d t}=P_{\mathrm{e}}(t)+P_{\mathrm{cw}, 1}(t)-P_{\mathrm{hw}}(t)-P_{\mathrm{loss}}(t)
$$

where $M$ is the water mass in the tank, $C_{p}$ denotes the specific heat capacity of water under constant pressure, $T$ stands for the tank water temperature, $P_{\mathrm{e}}$ signifies the thermal power output of the heating elements, $P_{\mathrm{cw}, 1}$ and $P_{\mathrm{hw}}$ refer to cold water inflow and hot water outflow of the tank, and $P_{\text {loss }}$ describes the heat losses.

The above first order differential equation defines the tank water temperature $T(t)$ at any time moment $t$ of the WH operation. By solving Equation 2.1 for $T(t)$, one can obtain the following expression:

$$
\begin{array}{r}
T(t)=\left(\frac{\alpha(t)}{\beta(t)}+T\left(t_{0}\right)\right) \exp \left[\beta\left(t-t_{0}\right)\right]-\frac{\alpha(t)}{\beta(t)}, \\
\alpha(t)=\frac{P_{\mathrm{e}}(t)+U A T_{\mathrm{amb}}+\dot{m}(t) C_{p} T_{\mathrm{cw}}}{M C_{p}}, \\
\beta(t)=-\frac{\dot{m}(t) C_{p}+U A}{M C_{p}},
\end{array}
$$




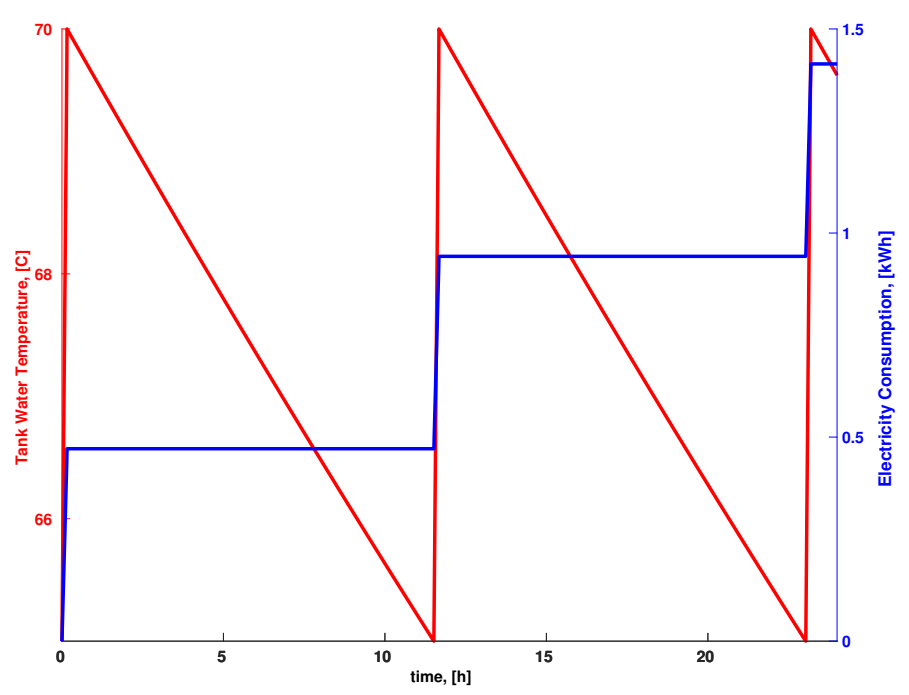

Figure 2.3: WH operation when tap is closed.

where $t-t_{0}>0$ is the time elapsed between the measurement of the tank water temperature $T\left(t_{0}\right)$ and $T(t), U A$ represents the heat loss coefficient, $T_{\mathrm{amb}}$ specifies the average ambient temperature, $\dot{m}(t)$ characterizes the mass flow rate from the tank at time $t, T_{\mathrm{cw}}$ alludes to the cold water temperature nearly constant during a day.

After re-writing Equation 2.2, we can derive the time needed to heat the water from $T\left(t_{0}\right)$ to $T(t)$ :

$$
t=\ln \left[\frac{\frac{\alpha(t)}{\beta(t)}+T(t)}{\frac{\alpha}{\beta}+T\left(t_{0}\right)}\right] .
$$

Because different terms appear differently in Equation 2.1 when the hot water tap is open or closed, we discriminate between these two cases.

\section{When TAP IS CLOSED}

When the hot water tap is closed, the water temperature inside the WH cycles within the deadband setpoint temperatures as depicted in Figure 2.3.

In case of a closed tap, Equation 2.1 can be spread out in two equations for the heating and cooling periods, respectively:

$$
M C \frac{d T}{d t}=\left\{\begin{array}{l}
P_{\mathrm{e}}-P_{\text {loss }}(t), \text { if } \mathrm{WH} \text { is on; } \\
-P_{\text {loss }}(t), \text { otherwise. }
\end{array}\right.
$$

\section{WHEN TAP IS OPEN}

Considering the hot water system in Figure 2.1, the hot water outflow from the tank $\dot{m}>0$ and the cold water inflow in the mixer $\dot{m}_{\mathrm{cw} 2}>0$ when a user opens the tap. 


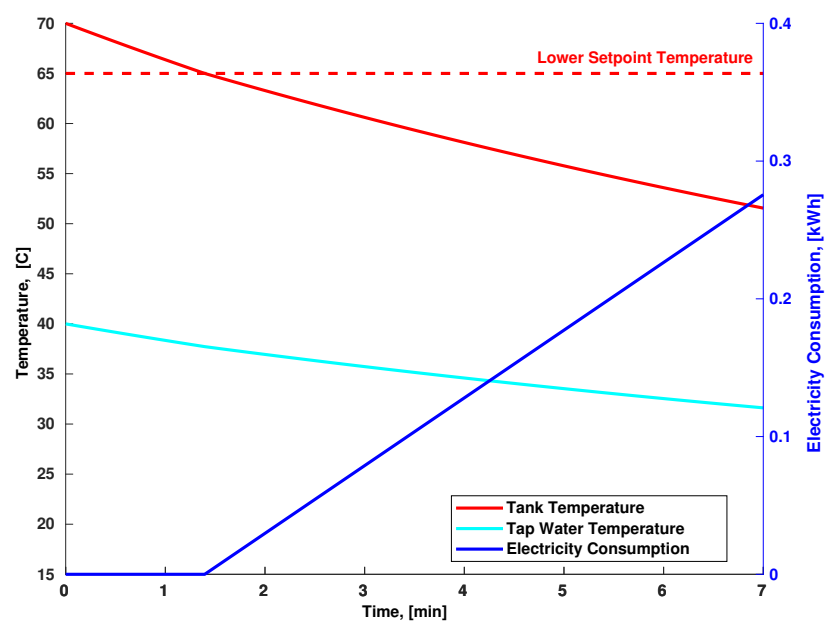

Figure 2.4: WH operation when tap is open.

Equation 2.1 then breaks down into two cases depending on the lower setpoint temperature $T_{\text {low SP }}$ :

$$
M C \frac{d T}{d t}=\left[\begin{array}{l}
P_{\mathrm{cw}, 1}-P_{\mathrm{hw}}(t)-P_{\mathrm{loss}}(t), \text { if } T(t)>T_{\mathrm{low} \mathrm{SP}} \\
P_{\mathrm{e}}+P_{\mathrm{cw}, 1}-P_{\mathrm{hw}}(t)-P_{\mathrm{loss}}(t), \text { otherwise. }
\end{array}\right.
$$

By manually tuning the tap mixer, a proportion the hot $\dot{m}$ and cold $\dot{m}_{\mathrm{cw} 2}$ flows can be regulated which allows a user to establish a comfortable tap water temperature $T_{\mathrm{d}}$ and water flow rate $\dot{m}_{\mathrm{d}}$. Energy and mass balances in the tap mixer can be written as:

$$
\left[\begin{array}{l}
P_{\mathrm{d}}=P_{\mathrm{hw}}+P_{\mathrm{cw} 2} \\
\dot{m}_{\mathrm{d}}=\dot{m}+\dot{m}_{\mathrm{cw}}
\end{array}\right.
$$

where $P_{\mathrm{d}}$ is the tap water thermal power demanded by the user, $P_{\mathrm{hw}}$ is the thermal power outflow from the tank, $P_{\mathrm{cw} 2}$ is the thermal power inflow of cold water in the mixer, and $\dot{m}_{\mathrm{d}}, \dot{m}, \dot{m}_{\mathrm{cw}}$ are the demanded, hot and cold water mass flow rates, respectively.

One can find from Equation 2.1 and Equation 2.6 that any outflow from the tap $\dot{m}_{\mathrm{d}}>$ $\left(P_{\mathrm{e}}-P_{\text {loss }}\right) /\left[C\left(T_{\mathrm{d}}-T_{\mathrm{cw}}\right)\right]$ leads to a decrease of the tank water temperature $T$ over time, causing a decline of the tap water temperature $T_{\mathrm{d}}$ as shown in Figure 2.4.

From Figure 2.4 one can see that the temperature $T_{\mathrm{d}}$ inevitably drops while the tap is open. The tap water temperature value $T_{\mathrm{d}}(t)$ at any instant of time $t$ can found from the system 2.6 while noticing that $P_{\mathrm{d}}(t)=\dot{m}_{\mathrm{d}}(t) C_{p} T_{\mathrm{d}}(t), P_{\mathrm{hw}}(t)=\dot{m}(t) C_{p} T(t)$ and $P_{\mathrm{cw} 2}=$ $\dot{m}_{\mathrm{cw} 2}(t) C_{p} T_{\mathrm{cw}}$ as it is shown below:

$$
T_{\mathrm{d}}(t)=\frac{\dot{m}(t)}{\dot{m}_{\mathrm{d}}(t)}\left(T(t)-T_{\mathrm{cw}}\right)+T_{\mathrm{cw}}
$$

When the temperature $T_{\mathrm{d}}(t)$ is lower than the minimum water temperature appreciated by a user, the user may experience the thermal discomfort. We argue that a negative impact 
on user comfort during the standard operation of the WH can be diminished when applying a different control strategy.

\subsubsection{HeAt Losses}

The heat losses have a negative impact on operational costs of a WH because the heat dissipated to the environment requires extra electricity to compensate it. The heat loss rate can be computed by the following equation:

$$
P_{\text {loss }}(t)=U A\left(T(t)-T_{\mathrm{amb}}\right),
$$

where $A\left[\mathrm{~m}^{2}\right]$ is the area of the WH jacket exposed to the environment; $U\left[\mathrm{~W} /\left(\mathrm{m}^{2} \mathrm{~K}\right)\right]$ stands for the overall thermal conductivity coefficient of the system "water-insulation-air".

Because the tank water temperature is often higher than the ambient temperature, the heat losses $P_{\text {loss }}(t)>0$ naturally lead to cooling of the tank water.

WHs exhibit a wide disparity of water heating energy efficiencies and stand-by lossses depending on the size and insulation of the WH tank amongst other factors. The influence of the heat loss coefficient $U A$ on energy consumption in case of a single 10-minute WA is shown in Figure 2.5. As it can be seen from Figure 2.5, the bigger the value of $U A$, the higher the electricity consumption.

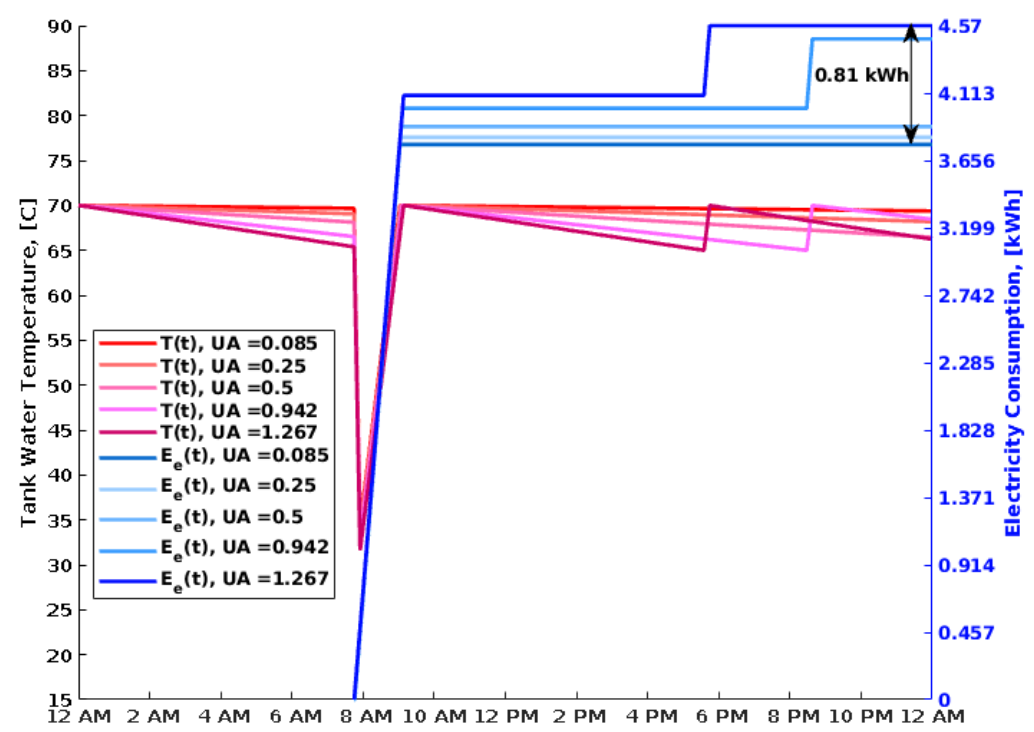

Figure 2.5: Effect of $U A$ on daily electricity consumption.

\subsection{Parameters of Water Heater Model}

Since we are focused on small-sized domestic WHs, we utilize technical parameters of the WH similar to a 80-liter model with the heating power 2950 [W] available in the market ${ }^{3}$ The adopted technical characteristics are present in Table 2.3.

\footnotetext{
${ }^{3}$ The water heater model used as a reference is a vertical cylindric Inventum EDR-80 [86].
} 
Table 2.3: Applied technical characteristics of the water heater:

\begin{tabular}{|c|c|}
\hline Rated Volume $M / \rho,[\mathrm{L}]$ & 80 \\
Heating power, $[\mathrm{W}]$ & 2950 \\
Water heating time $\left(15^{\circ} \mathrm{C}-88^{\circ} \mathrm{C}\right),[\mathrm{min}]$ & $139^{4}$ \\
$\mathrm{UA},[\mathrm{W} / \mathrm{K}]$ & 0.95 \\
Cold water temperature, $\left[{ }^{\circ} \mathrm{C}\right]$ & 15 \\
Ambient temperature, $\left[{ }^{\circ} \mathrm{C}\right]$ & 24 \\
\hline
\end{tabular}

The heat loss rate $P_{e} q t x$ loss in Equation 2.1 can be computed as:

$$
P_{\text {loss }}(t)=U A\left(T(t)-T_{\mathrm{amb}}\right),
$$

where $A\left[\mathrm{~m}^{2}\right]$ is the area of the WH jacket exposed to the environment; $U\left[\mathrm{~W} /\left(\mathrm{m}^{2} \mathrm{~K}\right)\right]$ stands for the overall thermal conductivity coefficient $U\left[\mathrm{~W} /\left(\mathrm{m}^{2} \mathrm{~K}\right)\right]$ of the system "waterinsulation-air".

Although Table 2.3 provides us with a reference to the water heating time that takes the $\mathrm{WH}$ to heat $80[\mathrm{~L}]$ of water from $15^{\circ} \mathrm{C}$ to $88^{\circ} \mathrm{C}$, finding the exact insulation coefficient $U A$ is not a trivial task. It is clear that we cannot simply substitute the heating time and the temperatures in Equation 2.3 to obtain the coefficient $U A$, because the equation is a model that only approximates experimentally obtained parameter in Table 2.3. We need to come up with another method to estimate the insulation coefficient $U A$.

One of the ways to determine the thermal conductivity $U A$ is to compute it analytically based on a given energy factor $E F[\%]$ of a WH. We adopt a computational method described in $[87,88]$ to find a possible range of the UA parameter to be used in our WH model. In this method, the following system of equations has to be solved for UA provided that all other parameters except UA and energy efficiency are given:

$$
\left[\begin{array}{l}
E F=\frac{Q_{\text {in }}}{l} Q_{\text {out }} \\
Q_{\text {in }}=\frac{M_{\mathrm{out}} C_{\mathrm{p}}\left(T-T_{\mathrm{cw}}\right)}{\eta_{\mathrm{r}}}\left(1-\frac{U A\left(T-T_{\mathrm{amb}}\right.}{P}\right)+24 U A\left(T-T_{\mathrm{amb}}\right) \\
Q_{\text {out }}=M_{\mathrm{out}} C_{\mathrm{p}}\left(T-T_{\mathrm{cw}}\right)
\end{array}\right.
$$

where $Q_{\text {in }}$ and $Q_{\text {out }}$ stand for the energy incoming and leaving the $\mathrm{WH} ; M_{\text {out }}$ is the mass of water withdrawn from the WH during the test time of 24 hours; $\eta_{\mathrm{r}}$ denotes the recovery efficiency ( 0.98 for electric water heaters).

After substituting the parameters listed in Table 2.3 in the system denoted by Equation 2.10, one can obtain the following approximation formula for the $\mathrm{WH}$ with power rate $2,95[\mathrm{~kW}]:$

$$
U A=\frac{30.894}{E F}-31.524\left[\mathrm{BTU} / \mathrm{hr}^{*} \mathrm{~F}\right]=\frac{16.297}{E F}-16.630 \mathrm{~W} / \mathrm{K} .
$$

An experimental study carried out by [89] exhibited a wide disparity of EF of WHs depending on the size and insulation amongst other factors as depicted in Figure 2.6.

\footnotetext{
${ }^{4}$ The declared heating time of Inventum EDR- 80 based on the specification.
} 


\begin{tabular}{llllllll}
\hline Unit \# & Unit Description & Rated & Power, & $\mathbf{E F}$ & $\eta \mathbf{r}$ & $\mathbf{U A}, \mathbf{W} / \mathbf{K}$ & Size \\
& & Volume, & $\mathbf{k W}$ & & & {$[\mathbf{B t u} /(\mathbf{h} * \mathbf{F})]$} & Category \\
& & $\mathbf{L}[\mathrm{gal}]$ & & & & & \\
& & $23[6]$ & 1 & 0.94 & 0.98 & $1.16[2.21]$ & POU \\
\hline \hline 1 & Electric Resistance & $114[30]$ & 4.5 & 0.95 & 0.98 & $0.48[0.91]$ & Low \\
2 & Electric Resistance & $189[50]$ & 4.5 & 0.90 & 0.98 & $1.35[2.56]$ & Medium \\
3 & Electric Resistance & $189[50]$ & 4.5 & 0.95 & 0.98 & $0.48[0.91]$ & Medium \\
4 & Electric Resistance & & & & & &
\end{tabular}

Figure 2.6: Values of EF, $\eta_{\mathrm{r}}$ and UA obtained by [89].

We consider a fleet of WHs with the energy factors EF in the range from 0.925 to 0.975 investigated by [88] so that based on Equation 2.11 the coefficient UA takes values $\in[0.085,0.975][\mathrm{W} / \mathrm{K}]$. In the following chapters, we utilize these values of the thermal conductivity coefficient $U A$ to evaluate the control efficiency on different models of WHs.

\subsection{SUMMARY}

This chapter provided an overview of the state-of-the-art control approaches for demand response (DR) of domestic electric tank water heaters (WHs).

Since the question of user comfort satisfaction is of a special interest in this thesis, we have made it a starting point of our review and have considered how the user comfort is respected by different DR solutions. We have shown that solutions differ a lot in the formulation of optimization problems and in the ways they are solved. Deterministic optimization approaches tangibly dominate over other approaches (e.g., probablistic) in the literature. The majority of optimization models attempts to control the states of the heating elements of the WH and thus utilizes the WH state as a control variable. Our observations in the scope of this work can be summarized as follows:

- about user comfort models:

- the tap water temperature and tap water flow rate (directly experienced by the user) are commonly not seen as variables in the user comfort modeling;

- user comfort is often represented by instantaneous values, while the duration of a temperature stimulus on a human body is renounced;

- the temperature deviations are not personalized, i.e. the factor that makes $5^{\circ} \mathrm{C}$ water temperature difference acceptable for one person and unacceptable for another is not present in modeling;

- a possible user thermal tolerance to uncomfortable water temperature is typically not a part of user comfort modeling;

- related to control:

- the minimization of heat losses is not explicitly considered as an optimization goal; 
- the hot water flow rate is not considered as a control variable in the reviewed literature, although the user-comfortable tap water temperature is a combination of both the temperatures and flows.

Most of the reviewed solutions ensure user comfort by maintaining a predefined amount of heat inside the $\mathrm{WH}$ even during the periods of no hot water usage. Taking into account that stand-by heat losses induce extra electricity consumption, a more energy efficient solution would be to heat the water only upfront WAs instead of keeping the tank water hot all the time between hot water activities (WAs). Also, people typically require distinct amounts of heat for different WAs, meaning that the stored heat might be not utilized completely during a WA and its residuals can contribute to the stand-by heat losses.

Furthermore, we explored the complexity of the user comfort representation. Based on the reviewed literature, we conclude that (a) the personal aspect of user comfort, (b) the user thermal tolerance, and (c) the time aspect of comfort are theoretically justified for consideration in the user comfort modeling.

Finally, we investigated different ways to model the WH operation. We examined the question of stratification in the WH tank and decided in favor of a well-mixed WH tank model explaining it by a number of factors relevant to this work. The well-mixed model of the WH was formalized and parameters of the model were defined. 



\section{3 User Comfort Modeling}

This chapter introduces the topic of user comfort in control of residential electric tank water heaters (WHs). By virtue of comfort modeling it is possible to determine the boundaries of the end-user comfort during the hot water usage. Incorporating these boundaries in control of WHs can allow for control actions with respect to user comfort, hence prevent user comfort deterioration.

In this chapter, we propose two user comfort models with regard to environmental parameters such as the tap water temperature and the tap water flow rate experienced by the user during the hot water usage. These are the user thermal comfort and the flow comfort models. Besides the environmental variables, the models include variables that specify how an individual perceives the tap water. The mapping between two types of variables is realized by means of the user tolerance functions. These functions relax hard constraints for the user comfort to be strictly within a predefined boundaries during the WH operation and allow for a gradual degradation of the user comfort around the desired user comfort zones. Such user tolerance exploited by control algorithms can allow for the operation of the WH exactly on the boundary of the user comfort. The integration of the user comfort models in the control of the WH will be demonstrated in the next chapters.

In home environments electrical devices provide inhabitants with valuable services, supporting them in day-to-day duties. In particular, electric tank water heaters (WHs) provide occupants with a hot water service that can be used during hot water activities (WAs) which involve direct contact of a human body with water. Examples of WAs are washing hands, taking a shower and many others.

People typically appreciate hot water service that fulfills their needs. If the tap water is too hot or cold, the user may find the hot water service uncomfortable.

Most often WHs have insufficient heating capacities to instantly heat the running water. Instead, they heat up the water inside the tank so that it can be used later on. It is obvious that in case of a huge request of hot water, such an operation may lead to the thermal discomfort for the user. Furthermore, such a deficit of hot water may also be caused by 
the heat losses in the periods when the WH is turned off. Such turn offs of the WH can be planned or unplanned.

An example of a planned turn off can be load curtailment programs (e.g., Direct Load Control [90]) where the energy company shuts down residential WHs to avoid fatal peaks of the electricity demand in the grid. On the other hand, the WH can be shut off due to unplanned electricity outages or even internal WH failures. Hot water usage during (or right after) the periods of the planned or unplanned WH disconnection from the power supply brings a risk of unsatisfactorily heated or cold tap water. Summarizing, lacking reserve of the hot water in the $\mathrm{WH}$ together with incapability of the $\mathrm{WH}$ to heat up the flowing water may result in the thermal discomfort to the user.

Oppositely to the cases of an unexpected user thermal discomfort, there are situations where the user may want to intentionally sacrifice his thermal comfort. For example, residents may want to purposely use the tap water at the lower temperatures in order to save electricity for water heating and, as a result, achieve money savings. Because of smaller amounts of the hot water to be withdrawn from the WH tank (lower energy demand), the WH will consume less electricity to heat the water in that case. Thus, a decrease of the user thermal comfort can also be a result of electricity and money savings.

Based on the above examples for WHs, we can highlight the following observations:

- the conventional control of WHs heats the water without awareness of a possible intense hot water usage and(or) high comfort demand;

- some control program that shuts off the WH, i.e. alters its conventional control, induces extra risks of deficit of the hot water;

- a rational usage of the available hot water resource can support the user comfort needs during the upcoming WAs;

- the user can achieve electricity and money savings by using the tap water at a lower temperature.

All these aspects may lead to the user thermal comfort deterioration.

Besides the tap water temperature, people also experience the tap water flow rate during WAs. To establish comfortable tap water flows the users can regulate the mixer tap. Some WAs may require intense tap water flows which lead to a fast drop of the tank water temperature as a consequence, there is a higher risk of the thermal discomfort for the user.

To maintain his thermal comfort, the user might reduce the tap water flow rate, thereby reducing the thermal energy demand and prolonging the availability of the hot water ${ }^{1}$. However, it can turn out that the new tap water flow rate has to be very small, causing inconvenience to the user. To account for the user dissatisfaction with possible variations of the tap water flow during WAs, we consider another indicator of the comfortability of the hot water service, i.e. the flow discomfort.

Unlike the previous examples of a planned flow discomfort, there might be also situations where the user encounters an unexpected low tap water flow caused by technical failures (e.g., a drop of the water pressure in the pipes).

${ }^{1}$ In any case, this might have only a temporary effect, because the tap water temperature continuously drops during WAs. 
By knowingly reducing the tap water flow rate, the user can not only avoid a complete hot water cutoff, but also can achieve electricity, water and money savings because of a smaller amount of water that passes through the WH. This can also be done in an automated way, for example, by means of low-pressure faucets [91] and modern digital taps [92, 93].

Based on the examples of the user flow discomfort, we notice the following:

- the reduced tap water flow can support the user thermal comfort, if the user intentionally reduces the tap water flow rate;

- the user can save water, electricity and money at the cost of a reduced tap water flow.

To account for situations where the user may experience a thermal and(or) flow discomfort, there is a need for user comfort modeling.

In this chapter, we propose two comfort models that allow for the user comfort quantification in the residential hot water systems with WHs, namely the thermal comfort model and flow comfort model. By using the input information about the tap water utility and the occupants, the models can estimate (or reflect) a subjective feeling of the user comfort. Two models account respectively for the tap water temperature and tap water flow rate as descriptors of the water environment the user interacts with. While these environmental variables are external to the user, i.e. they come from the environment the user is exposed to, personal variables of the models describe individual user perception of the tap water. The personal variables include the user tolerances to the tap water temperature and to the water flow rate. Since the user comfort is, in essence, much more complex than that, the output of the proposed comfort models represents only a rough estimate of a real user feeling of comfort. The application of the thermal comfort model will be demonstrated in the next chapters.

\subsection{Premises for User Comfort Modeling}

This section discusses attributes of the residential hot water consumption and human comfort that can pave the way for user comfort modeling during hot water activities.

To begin with, we refer to features inherent to the hot water usage at home. Further, we outline the factors that influence the user feeling of comfort during hot water usage and how they can be accounted for in the user comfort modeling.

\subsubsection{Specifics of Human Comfort during Hot Water Usage}

A human feeling of comfort is very individual and therefore very complex. When people have contact the tap water, they typically perceive and appreciate such physical properties as the water temperature and the water flow rate. However, the ways they interpret these proporties as comfortable or uncomfortable hinges on numerous factors, which obstructs the user comfort modeling.

First and foremost, people demonstrate a highly dynamic and spontaneous behavior and so are their comfort preferences. The hot water usage can be highly dynamic and intermittent in time as it can consist of multiple events when the hot water tap is open/closed (e.g., pauses for soaping when taking a shower). 
Secondarily, because people can perform different water activities (WAs) in different water temperature ranges and flow rates, their comfort expectations for distinct WAs can also differ. For example, individuals can usually wash their hands in a wider range of the tap water temperatures (e.g., $15 \ldots 45^{\circ} \mathrm{C}$ ) than during a shower. Also, some WAs that involve direct hand contact with water can be accomplished only in certain temperatures, as, for instance, a 'washing dishes by hand' WA may be hard to accomplish in the cold water.

Furthermore, the user comfort is not static and can change over the time for the same individual and the same WA. Thus, a person will, most likely, desire to take a shower with different temperatures after he wakes up in the morning and after an intensive jogging. If a person feels uncomfortable tap water temperature (or water flow) during a WA, his discontent can, possibly, grow over the time so that the user can interrupt the WA at a certain point without accomplishing it.

Moreover, a range of comfortable water temperatures during the same WA is influenced by external variables such as the indoor and outdoor temperatures, air humidity, etc.

The user comfort modeling for the hot water service has its own specifics as contrasted to other applications (e.g., comfort modeling for space heating [94-96]. People interact with the tap water during WAs (e.g., showering) by having a direct body-contact (not protected by clothing). Furthermore, people might interact with water only locally with different parts of the body (e.g., washing hands). The body-contact with water has a temporary character, since water is used during activities (e.g., washing dishes) that have a finite duration.

Last but not the least, comfort preferences are subjective, meaning that they might vary from one person to another. For example, talking about people of different age groups, the skin sensitivity of children is commonly higher than for adults [97]. Which means that children typically perform WAs (e.g., taking shower) in cooler water temperatures than adults. For example, water temperature at $49^{\circ} \mathrm{C}$ can cause second degree full thickness skin burn for adults after 10 minutes of exposure [42, 98], while for children it approximately takes a quarter of that time [97, 99].

The specifics of the human comfort during WAs can be summarized as follows:

- an individual is in a direct body-contact with water during WAs meaning that (s)he experiences and appreciates the physical properties of water directly with his body;

- the user comfort can dynamically change over the time for the same person and for the same WA performed in different long-distant time frames (e.g., taking a shower in the morning and in the evening) and shorter time frames (e.g., before and after soaping);

- people can, possibly, feel an increase of discomfort over the time which can force them to take actions for comfort improvement (e.g., by adjusting the mixer tap) and(or) interrupt their WAs;

- external variables not related to the user and the hot water service may influence user feeling of comfort;

- a person may have only a local contact with water without exposing his whole body (e.g., washing hands); 
- hot water is used to accomplish some WAs that have a finite duration;

- a feeling of comfort is very individual, meaning that it is influenced by various personal physical, physiological, psychological, and other factors and hence typically differs for different people.

\subsubsection{Factors Influencing User Comfort}

In a set of experiments done in the mid 60's [100] a group of 26 people was exposed to a constant temperature in a water bath to measure their cutaneous thermal sensation. It has been found that individual thermal sensation can vary depending on the internal temperature of the body. People sense the ambient temperature as agreeable if it leads to normalization of the internal temperature of the body. That is why the overheated (hyperthermic) body perceives cold water as pleasant and hot water as very unpleasant, while cold (hypothermic) body feels it opposed. A feeling of a thermal pleasantness was explored in experiments with hot water environments in the mid 90's [101]. During the experiments it was found that participants felt pleasant when they stayed for some time in a hot spa and then were exposed to a cooler temperature of the surroundings to reduce thermal stress. It was concluded that the time during which an individual spends in a certain thermal environment is a key factor for his thermal satisfaction. Thermal comfort was identified as a dynamic process that evolves in time and can take distinct states like feeling of warm, comfortably warm, comfortably cool water and other. Furthermore, when a human experiences thermal discomfort, any reduction of the thermal stress is sensed as pleasant during the temperature transition.

Based on these experiments, we can underline the following factors that play role in a human perception of the water temperature:

- personal variables such as the internal temperature of a human body responsible for thermal regulation in the body;

- the time of exposure of a human body to the tap water.

While the question how an individual experiences the comfort depends on the psychophysiology of an individual (e.g., the mood) which cannot be measured, other parameters of the human comfort seem to be possible to represent numerically.

\subsubsection{Environmental and Personal Variables}

Talking about the factors that can be measured and accounted for (to some extent) in the user comfort modeling, the thermal comfort during WAs can be represented by two components, namely the environmental and personal variables, by analogy to the comfort modeling approach for space heating discussed in Chapter 2.

Considering the environmental variables, the ambient conditions such as the ambient air temperature, mean radiant temperature, air velocity and humidity may be considered as variables controlled by external devices (e.g., a space heater). Since we only focus on the system WH-user, we neither can control such external variables, nor estimate their influence on the user comfort during WAs. For simplicity, we assume that these external variables are always maintained at the user-desired comfort level and thus can be neglected in comfort modeling during hot water usage. 
People are typically satisfied with the tap water when they get the water at the desired temperature and when the tap water flow rate is within the preferred range. These variables sufficiently describe the user ambient during WAs and are controllable in the context of this research. Therefore, the tap water temperature and tap water flow rate can be utilized as the environmental variables for the user comfort modeling. Since these two variables are different by nature, their impacts on the user comfort are different too. In that light, one may want to distinguish between the two types of comfort, namely the thermal comfort and flow rate comfort, by modeling them separately.

While the environmental variables describe factors external to a human body, personal variables are directly binded to a human physiology. There are numerous indicators in a human body that may influence the user feeling of comfort during WAs. Examples of those are the internal temperature of the body, skin temperature, metabolic rate, skin density and thickness, specific heat of tissues, blood flow, concentration of melanin and the water content.

As opposed to the environmental variables, the personal variables can highly vary from one person to another as well as they can change for the same person with time, which obstructs their estimation in practice. Some of them can also depend on activities performed by an individual before hot water usage and his previous exposure to a (possibly) different environment as in the case of the internal temperature of the body, skin temperature and metabolic rate.

Other personal variables can be expressed through the other variables that can represented them in a more "aggregated" (or abstract) way. For example, the thermal conductivity of the body hinges, amongst others, on the parameters such as density, thickness and specific heat of tissues [102].

Different personal variables can have different variability in time. For instance, the thermal conductivity of the body depends on long-term physiological processes and thus does not change often, however, varies across the human body [103]. The thermal conductivity has been extensively used in many comfort models for space heating [71, 94, 96, 104].

Another potential candidate to consider in the user comfort modeling can be thermal receptors ${ }^{2}$ of the human body. How a person feels hot and cold water (thermal sensation) depends, amongst others, on nerve endings in the skin, some of which respond to warm stimuli while others respond to cold [71]. Noticeably, the mechanisms of the nerve thermal sensation (frequency response, temperature adaptation, pain perception and other) do not frequently change for one person over time, whereas measurements obtained experimentally for groups of individuals do not represented high variations [74]. As it follows from the thermal physiotherapy theory (refer to Chapter 2), the thermal receptors are activated in certain temperature ranges and begin to fire with specific frequencies. There exist temperature ranges where the thermal receptors are firing with the same frequencies. An individual will not distinguish two cold (or two hot) temperature values if they have the same frequencies. From this, a wider temperature range of identical firing frequencies corresponds to a wider thermal tolerance zone in which any temperature is felt as equally comfortable to the user.

${ }^{2}$ To the best of the author's knowledge, the thermal receptors were barely considered in the user comfort modeling in the literature. 


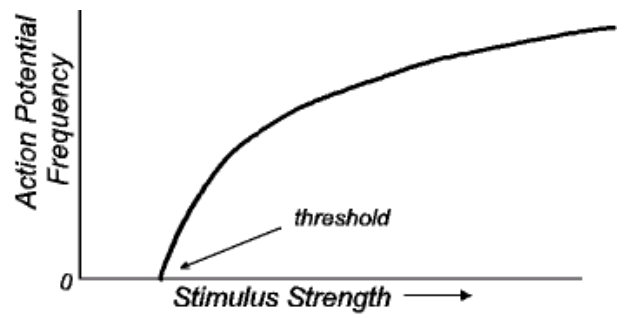

Figure 3.1: Activation of mechanoreceptors (adapted from [107]).

Apart from the thermal receptors in a human body, there are also receptors responsible for sensing the mechanical pressure and distortion, so-called mechanoreceptors. There are 4 types of mechanoreceptors located in epidermis and dermis layers of the skin which respond to a range of frequencies of mechanical stimulation in the range from 0.3 to 500 $\mathrm{Hz}$ [105]. Two of these receptor types ("Merkel receptor" active in the range $0.3-3 \mathrm{~Hz}$ and "Pacinian corpuscle" in the range $10-500 \mathrm{~Hz}$ ) are responsible for perception of the steady-state pressure and vibration (changing stimulation) [106]. Considering typical domestic WAs where the user interacts with the running water, the water flow does not usually change faster than 3 times per second, but rather remains fixed after the user sets the comfortable tap water flow at the tap mixer at the beginning of the WA. Which means that the user perceives the tap water flow via a population of Merkel receptors during WAs. Merkel receptors change the firing frequency mainly based on the stimulus strength (force) and the area it is applied to, i.e. the activate due to the mechanical pressure. The graph in Figure 3.1 illustrates the relationship between the size of the mechanical stimulus and the frequency of action potentials generated by a mechanoreceptor.

As can be seen from Figure 3.1, there exist a mechanical threshold that indicates the amount of force required to activate the sensory receptor. Because of this threshold for mechanoreceptor activation (see Figure 3.1), an individual may feel some tolerance to the amount of stimulating force caused by the tap water flow. Since the activation threshold in mechanoreceptors may vary based on the frequency and amplitude of stimulation [108], one can draw a conclusion that an individual may have a distinct tolerance to varied tap water flows. Although there is a variation in concentration of mechanoreceptors across the human body, the mechanisms of their response to external stimulation does not change for different people.

Even though the nerve sensitivity coefficients vary for different body areas (the face has the highest and the lower part of the legs has the lowest sensitivity), it seems possible to account for that variation considering involvement of different parts of the body in different WAs. Thus, for example, washing hands may typically engage fingers, palms, wrist and forearm which have comparable sensitivity coefficients, while taking a shower may be considered as a compound activity with a superposition of multiple sensitivity coefficients. Moreover, it appears feasible to perform field experiments where participants are asked to reflect their feedback on a thermal sensational scale (e.g., on the scale ranging from painfully hot to painfully cold as in Figure 2.2). 


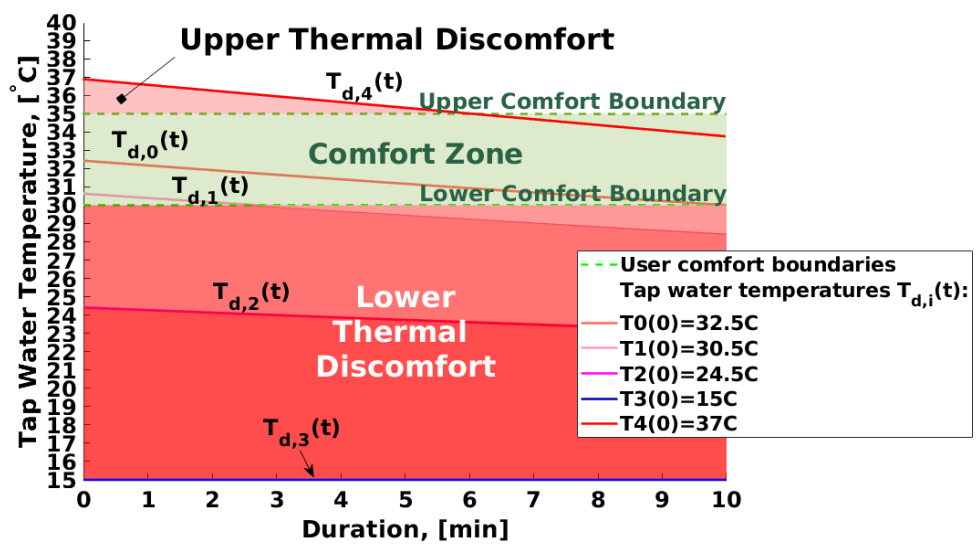

Figure 3.2: Possible thermal discomfort zones during a single WA.

\subsection{The rmal Comfort Modeling}

A user may experience the thermal discomfort originating from a mismatch between the tap water temperature comfortable to the user and the water temperature actually supplied to him.

To give an insight into the user thermal (dis)comfort, let us consider a scenario of a WH with a partially discharged heat in the tank (e.g., due to the preceding hot water usage) and a person who performs a 10-minute WA in a tap water. The person considers the tap water temperature in the range of $T_{\exp }(t) \in[30,35]^{\circ} \mathrm{C}$ as fully comfortable. For simplicity, let us assume that all the tap water comes directly from the tank without being mixed with cold water in the mixer tap, i.e. $T_{\mathrm{d}}(t)=T(t)$. Furthermore, suppose that the tap water flow rate $\dot{m}_{\mathrm{d}}(t)=\dot{m}_{\exp }(t)=$ const, since the tap flow $\dot{m}_{\mathrm{d}}(t)$ typically remains fixed during domestic WAs, i.e. once the water flow is set at the beginning of a WA it is commonly not changed till the WA is finished. Five cases of hot water usage ${ }^{3}$ that correspond to different initial tank water temperatures $T_{i}(0)$ are shown in Figure 3.2.

At any moment $t$ during the WA, the user may experience the thermal discomfort arising from the tap water temperature $T_{\mathrm{d}}(t)$ (a) under the Lower Comfort Boundary $T_{\text {c.min }}=30^{\circ} \mathrm{C}$, or (b) above the Upper Comfort Boundary $T_{\text {c.max }}=35^{\circ} \mathrm{C}$. Such temperature deviations can be graphically represented by the shaded regions between the curve $T_{\mathrm{d}}(t)$ and the comfort boundaries $T_{\text {c.min }}$ and (or) $T_{\text {c.max }}$ as depicted in Figure 3.2. Obviously, the bigger the shaded region, the bigger thermal discomfort experienced by a user. We refer to the regions between $T_{\mathrm{d}}(t)$ and $T_{\text {c.min }}$ as the Lower Thermal Discomfort. As opposed, the regions between $T_{\mathrm{d}}(t)$ and $T_{\text {c.min }}$ are referred to as the Upper Thermal Discomfort.

In case of $T_{0}(0)=32.5^{\circ} \mathrm{C}$, the $\mathrm{WH}$ has sufficient heat to provide the water temperature comfortable to the user during the whole WA $\left(T_{\mathrm{d} 0}(0)=32.5^{\circ} \mathrm{C}\right.$ and $\left.T_{\mathrm{d} 0}(10)=30^{\circ} \mathrm{C}\right)$. However, if $T_{1}(0)=T_{\mathrm{d} 1}(0)=30.5^{\circ} \mathrm{C}$, the user starts to experience the inconvenient water temperature after $3[\mathrm{~min}]$ from the moment the tap is opened. In another case where $T_{2}(0)=T_{\mathrm{d} 2}(0)=$

${ }^{3} \mathrm{~A}$ well-mixed model of the WH described in Section 2.5 .2 is employed hereinafter in all the simulations. 
$24.5^{\circ} \mathrm{C}$ ), the user gets the uncomfortable water temperature during the entire WA. The highest thermal discomfort will be felt by the user when the tank is filled with cold water $T_{\mathrm{d} 3}=T_{3}=T_{\mathrm{cw}}=15^{\circ} \mathrm{C}$. These three cases $\left(T_{\mathrm{d} 1}-T_{\mathrm{d} 3}\right)$ describe the situations where the user gets the tap water at the temperature lower than the desired one. In general, it might be the case that the user can receive uncomfortably high water temperature while performing the WA (e.g., very hot water remains in the pipe from the previous WA). Thus, in case of $T_{\mathrm{d} 4}(0)=T_{4}(0)=37^{\circ} \mathrm{C}$, the user senses the higher temperature than expected during the first 6 [min] of the WA.

In some cases, the temperature boundaries that restrict the comfort zone can be functions of time $\left[T_{\text {c.min }}(t), T_{\text {c.max }}(t)\right]$, while in other cases they may be fixed $\left[T_{\text {c.min }}, T_{\text {c.max }}\right]$ during a single WA (as in Figure 3.2). Moreover, there may be situations where the comfort zone is narrowed down to a single function $T_{\text {c.min }}(t)=T_{\text {c.max }}(t)$ (e.g., when taking a shower). Then, any tap water temperature $T_{\mathrm{d}}(t) \neq T_{\text {c.min }}(t)$ results in the thermal discomfort to the user.

Intuitively one can notice that not only instantaneous water temperature changes can matter to the user, but also the time during which the user endures them. If the user feels a slight lasting deviation of the water temperature from the thermal comfort zone, he can experience as much discomfort as after a big short temperature mismatch. Therefore, thermal discomfort may increase over the time.

To account for both instantaneous temperature deviations and their durations during a single WA, one can appeal to the cumulative difference between the actually supplied water temperature $T_{\mathrm{d}}(t)$ and the Lower Comfort Boundary $T_{\text {c.min }}(t)$ (and(or) the Upper Comfort Boundary $T_{\text {c.min }}(t)$ ), as given in Equation 3.1 for the case where $T_{\exp }(t)=T_{\text {c.min }}(t)=$ $T_{\text {c.max }}(t)$.

$$
A_{\mathrm{T}}(t)=\left|\int_{0}^{t} T_{\exp }(t)-T_{\mathrm{d}}(t) d t\right|,
$$

where $A_{\mathrm{T}}(t)$ is the area between the curves $T_{\exp }(t)$ and $T_{\mathrm{d}}(t)$ in the range $[0, t]$ as a function of time $t$.

As can be seen from Equation 3.1, if $T_{\exp }(t)>T_{\mathrm{d}}(t)$, the user experiences the Lower Thermal Discomfort. In case $T_{\exp }(t)<T_{\mathrm{d}}(t)$, the user encounters the Upper Thermal Discomfort.

In general, the user comfort zone can be defined by a range of temperatures $\left[T_{\text {c.min }}, T_{\text {c.max }}\right]$, $T_{\text {c.min }}<T_{\text {c.max. }}$. In that case, the expression for $A_{\mathrm{T}}(t)$ becomes twofold, as shown by Equation 3.2.

$$
A_{\mathrm{T}}(t)=\left[\begin{array}{l}
\int_{0}^{t} T_{\mathrm{c} \cdot \min }(t)-T_{\mathrm{d}}(t) d t, \text { if } T_{\mathrm{d}}(t)<T_{\mathrm{c} \cdot \min }(t) ; \\
\int_{0}^{t} T_{\mathrm{d}}(t)-T_{\mathrm{c} . \max }(t) d t, \text { if } T_{\mathrm{d}}(t)>T_{\mathrm{c} \cdot \max }(t) .
\end{array}\right.
$$

In practice, the tank water can be much hotter than the expected water temperature $T_{\exp }(t)<T(t)$, so that the user can get the desired water temperature by adjusting the mixer tap. Taking into account that there may be multiple periods during a single WA when $T_{\mathrm{d}}(t)<T_{\text {c.min }}$ and $T_{\mathrm{d}}(t)>T_{\text {c.max }}$ due to the tap adjustments, Equation 3.2 can be rewritten as follows:

$$
A_{\mathrm{T}}(t)=\int_{0}^{t} \max \left\{0, T_{\mathrm{c} \cdot \min }(t)-T_{\mathrm{d}}(t), T_{\mathrm{d}}(t)-T_{\mathrm{c} \cdot \max }(t)\right\} d t .
$$


It is clear that Equation 3.3 is far from reflecting the real user (dis)satisfaction with the temperature $T_{\mathrm{d}}(t)$, as it only considers a single environmental variable, i.e. the tap water temperature $T_{\mathrm{d}}(t)$, without accounting for the personal variables such as the skin sensitivity, skin temperature, metabolic rate and other that influence the user comfort too as discussed in Section 3.1.3.

To model the user thermal comfort more accurately, we need to take into consideration the personal feeling of the water temperature deviations. As outlined in Section 3.1.3, there is a vast majority of personal variables that affect user thermal comfort. This can dramatically complicate our user thermal comfort model. Instead, we utilize the user thermal tolerance as a representative of the comfort personal variables in our thermal comfort model. Being a more abstract parameter than the personal variables, the user thermal tolerance ${ }^{4}$ delineates the user ability to tolerate the tap water temperature deviations from comfortable ranges while experiencing dissatisfaction.

In our approach, we model the user tolerance to the water temperature via a piece-wise linear function as follows:

$$
F_{\mathrm{T}}\left(T_{\mathrm{d}}, t\right)=\left\{\begin{array}{l}
\alpha_{1}(t)\left[T_{\mathrm{d}}(t)-T_{\mathrm{c} . \min }(t)\right]+\beta_{1}(t), \text { if } T_{\mathrm{d}}(t) \in \Delta_{\mathrm{tol}}^{-}(t) \\
\alpha_{2}(t)\left[T_{\mathrm{d}}(t)-T_{\mathrm{c} \cdot \max }(t)\right]+\beta_{2}(t), \text { if } T_{\mathrm{d}}(t) \in \Delta_{\mathrm{tol}}^{+}(t) \\
0, \text { if } T_{\mathrm{d}}(t) \in\left[T_{\mathrm{c} . \min }(t), T_{\mathrm{c} \cdot \max }(t)\right] \\
1, \text { otherwise; }
\end{array}\right.
$$

where $\alpha_{1}(t) \leq 0, \beta_{1}(t) \geq 0$ and $\alpha_{2}(t) \geq 0, \beta_{2}(t) \geq 0$ are the coefficients that describe the intensity of the thermal comfort loss (decay); $\Delta_{\text {tol }}^{+}$and $\Delta_{\text {tol }}^{-}$specify the water temperature ranges tolerated by the user.

The user thermal tolerance function $F_{\mathrm{T}}$ in Equation 3.4a - Equation 3.4d is a loss function that describes the loss of the user thermal comfort per deviation of the temperature $T_{\mathrm{d}}(t)$ from the comfort boundaries $T_{\text {c.min }}(t)$ or $T_{\text {c.max }}(t)$ at time $t$.

The function $F_{\mathrm{T}}$ takes the values from 0 to 1 , meaning that the user experiences the zero thermal discomfort if $T_{\mathrm{d}}(t) \in\left[T_{\text {c.min }}(t), T_{\text {c.max }}(t)\right]$, and the user feels the temperature difference at the maximum level equal to 1 when $T_{\mathrm{d}}(t)$ is outside the margins $\Delta_{\text {tol }}^{+}(t)$ and $\Delta_{\text {tol }}^{-}(t)$. More precisely, Equation 3.4a requires $0<\alpha_{1}(t)\left[T_{\mathrm{d}}(t)-T_{\mathrm{c} . \min }(t)\right]+\beta_{1}(t)<1$ in case $T_{\mathrm{d}}(t) \in\left(T_{\text {c.min }}(t)-\Delta_{\text {tol }}^{-}(t), T_{\text {c.min }}(t)\right)$ and $\Delta_{\text {tol }}^{-}(t)>0$. Similarly, Equation $3.4 b$ requires $0<\alpha_{2}(t)\left[T_{\mathrm{d}}(t)-T_{\text {c.max }}(t)\right]+\beta_{2}(t)<1$ in case $T_{\mathrm{d}}(t) \in\left(T_{\text {c.max }}(t), T_{\text {c.max }}(t)+\Delta_{\text {tol }}^{+}(t)\right)$ and $\Delta+$ tol $(t)>0$. Furthermore, we allow the coefficient $\alpha_{1}(t)$ to be either $\alpha_{1}(t)=-\frac{1}{\Delta_{\text {tol }}^{-}(t)}<0$ if $\beta_{1}(t) \geq 0$, or $\alpha_{1}(t)=0$ when $\beta_{1}(t)>0$. In the latter case, the function $F_{\mathrm{T}}$ takes the form of a flat line in the range $\left[T_{\text {c.min }}(t)-\Delta_{\text {tol }}^{-}(t), T_{\text {c.min }}(t)\right)$. The same holds for the range $\left(T_{\text {c.max }}(t), T_{\text {c.max }}(t)+\right.$ $\left.\Delta_{\text {tol }}^{+}(t)\right]$ in case $\alpha_{2}(t)=0$ and $\beta_{2}(t)>0$. For example, the function $F_{\mathrm{T}}$ in case $\alpha_{1}(t)<0, \beta_{1}(t)=$ $0, \alpha_{2}(t)>0$ and $\beta_{2}(t)=0$ is depicted in Figure 3.3 for the moment of time $t$.

\footnotetext{
${ }^{4}$ The existence of the user thermal tolerance can be justified by the similar firing frequency of the thermal receptors in a certain ranges of water temperature, as discussed in Section 3.1.3.
} 


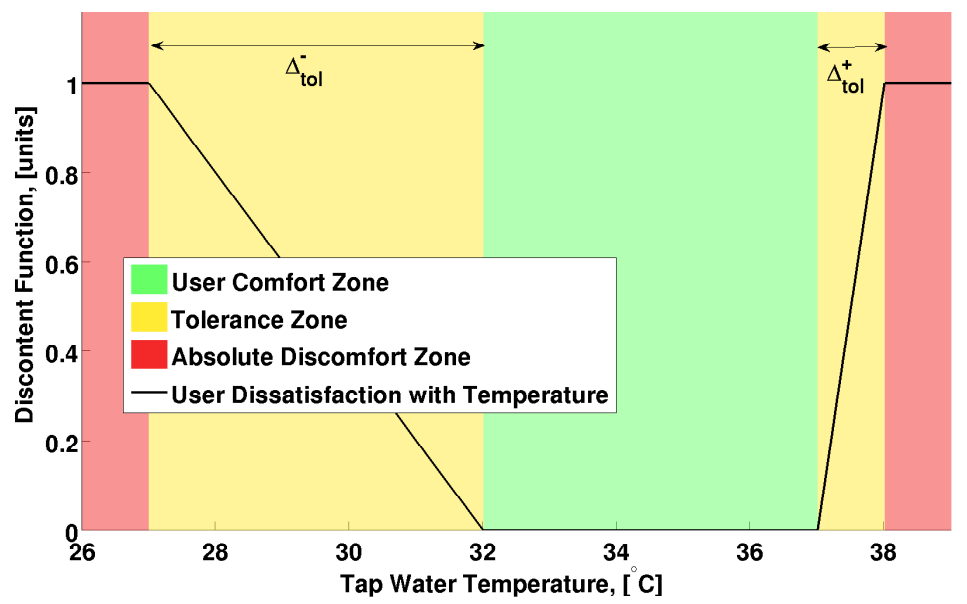

Figure 3.3: User thermal tolerance function $F_{\mathrm{T}}\left(T_{\mathrm{d}}, t\right)$ at time $t$.

We used a linear relationship to describe the dependency of the comfort loss from the tap water temperature deviations in function $F_{\mathrm{T}}$. Ideally, the shape of the tolerance function should come from the user input. In general, the function $F_{\mathrm{T}}$ is also a function of time $t$, meaning that the user thermal tolerance zones $\Delta_{\text {tol }}^{+}(t), \Delta_{\text {tol }}^{-}(t)$ as well as the coefficients $\alpha_{1}(t), \alpha_{2}(t), \beta_{1}(t), \beta_{2}(t)$ can all vary over the time. However, it seems unrealistic to question the user for such a complex time-varying input. We, therefore, simplify the expression in Equation 3.4a-Equation $3.4 \mathrm{~d}$ by applying the fixed coefficients during a single WA, i.e. $\alpha_{1}(t)=$ const, $\alpha_{2}(t)=$ const,$\beta_{1}(t)=$ const and $\beta_{2}(t)=$ cons $t$.

Different people usually have different comfortable tap water temperature ranges that can also vary for different WAs. Due to a vast diversity of domestic WAs and user thermal tolerance functions associated with them, it seems hard to model the thermal comfort of every single person per each individual WA. Instead, we assume that every $i$-th user has tolerance functions $F_{\mathrm{T}, i, j}$ associated with a certain $j$-th category of WAs (e.g., hygiene, cleaning, cooking, etc.). In this way, each thermal function $F_{\mathrm{T}, i, j}$ incorporates the personal comfort variables in the context of a certain WA category performed by the user.

By combining this contextual information with the expression in Equation 3.3, we can consider both the environmental and personal variables of the user thermal comfort in a context of the WA categories. The resulting expression $D_{\mathrm{T}}(t)_{i, j}$ that describes the thermal discomfort of the $i$-th user during a single WA belonging to the $j$-th category of WAs can be written as follows:

$$
D_{\mathrm{T}}(t)_{i, j}=F_{\mathrm{T}}\left(T_{\mathrm{d}}\right)_{i, j} A_{\mathrm{T}}(t)_{i, j}
$$

where $A_{\mathrm{T}}(t)_{i, j}$ is the area between the tap water temperature $T_{\mathrm{d}}(t)$ experienced by the $i$-th user at time $t$ (during a single WA of the category $j$ ) and the lower and(or) upper comfort boundaries $\left[T_{\text {c.min }}, T_{\text {c.max }}\right]$.

The expression $D_{\mathrm{T}}(t)_{i, j}$ in Equation 3.5 respects both the user comfort personal variables via the term $F_{\mathrm{T}}\left(T_{\mathrm{d}}\right)$ and the environmental variable (i.e. the tap water temperature $T_{\mathrm{d}}(t)$ ) by means of the term $A_{\mathrm{T}}(t)$. 
On the grounds that the expression $D_{\mathrm{T}}(t)_{i, j}$ in Equation 3.5 treats the user (dis)comfort through a simplified abstraction $F_{\mathrm{T}}\left(T_{\mathrm{d}}\right)_{i, j}$ of the real personal variables, $D_{\mathrm{T}}(t)_{i, j}$ can only nearly characterize the real thermal comfort of the user.

\subsection{Flow Comfort Model}

Apart from the tap water temperature, people experience the influence of the tap water flow on their bodies during hot water activities (WAs). In some situations it might be favorable for the user to limit the tap flow rate during WAs.

Firstly, intense tap water flows can cause the tank water temperature to plummet rapidly during WAs. As a result, the user can soon face a lack of hot water in the $\mathrm{WH}$ and experience the thermal discomfort. By reducing the tap water flow rate, the hot water service can be available for an extended period of time. Secondly, reduction of the tap water flow results in electricity and money savings due to the reduced thermal energy demand. Finally, this leads to the reduced amount of water passing through the household water meter and thus brings water savings. Despite the advantages of such a control action, the impact of the new tap flow rate on the user comfort is unknown. In case the new tap water flow is too low, the end-user might experience dissatisfaction with the hot water service, i.e. feel the flow discomfort.

Let us illustrate how the reduced tap water flow can facilitate the user thermal comfort. Consider a WH with a partially discharged heat in the tank (e.g., due to the preceding hot water usage). Suppose the user desires to fulfill a 10-minute WA in a comfortable tap water temperature range $\left[T_{\text {c.min }}, T_{\text {c.max }}\right]=[23,27.5]^{\circ} \mathrm{C}$ while having the tap water flow rate $\dot{m}_{\exp }=10[\mathrm{~L} / \mathrm{min}]$.

In case the user sets the tap water flow rate ${ }^{5}$ to the desired $\dot{m}_{\mathrm{d}}=\dot{m}_{\mathrm{exp}}=10[\mathrm{~L} / \mathrm{min}]$, he will experience the thermal discomfort equivalent to the tap water temperature drop $\Delta T_{\mathrm{d}}=2^{\circ} \mathrm{C}$ by the end of the WA as shown in Figure 3.4. Alternatively, if the tap water water flow rate is reduced by $3[\mathrm{~L} / \mathrm{min}]$ during the WA, the user comfort requirement would be fulfilled $\left(T_{\mathrm{d}}(10)=T_{\text {c.min }}=23^{\circ} \mathrm{C}\right)^{6}$. However, having the reduced tap water flow rate might be uncomfortable to the user. Thus, the question about the impact of the tap water flow rate on the end-user comfort during WAs can arise.

From the above example, one may presume that the time during which the user experiences the undesirable water flow plays a role to the user. By analogy to the tap water temperature in Section 3.2, persisting deviations of the delivered water flow rate $\dot{m}_{\mathrm{d}}$ from the user-expected tap water flow rate $\dot{m}_{\text {exp }}$ can be represented by a cumulative difference as follows:

$$
A_{\dot{m}_{\mathrm{d}}}(t)=\left|\int_{0}^{t} \dot{m}_{\mathrm{exp}}(t)-\dot{m}_{\mathrm{d}}(t) d t\right|,
$$

where $A_{\dot{m}_{\mathrm{d}}}(t)$ is the area between the graphs $\dot{m}_{\exp }(t)$ and $\dot{m}_{\mathrm{d}}(t)$ in the range $[0, t]$ as a function of time $t$.

Similar to the tap water temperature, the user might, in general, tolerate some variations of the tap water flow $\dot{m}_{\mathrm{d}}(t) \in\left[\dot{m}_{\mathrm{c} . \min }(t), \dot{m}_{\mathrm{c} . \max }(t)\right]$ without feeling them uncomfortable.

\footnotetext{
${ }^{5}$ In this example, we consider the tap water flow rate fixed within the WA $\dot{m}_{\mathrm{d}}(t)=\dot{m}_{\mathrm{d}}=$ const .

${ }^{6}$ Here, the reduced tap water flow rate will also lead to $3[\mathrm{~L} / \mathrm{min}] \times 10[\mathrm{~min}]=30[\mathrm{~L}]$ water savings and electricity savings induced by the reduced by $2[\mathrm{~L} / \mathrm{min}] \times 10[\mathrm{~min}]=20[\mathrm{~L}]$ amount of water passing through the WH.
} 

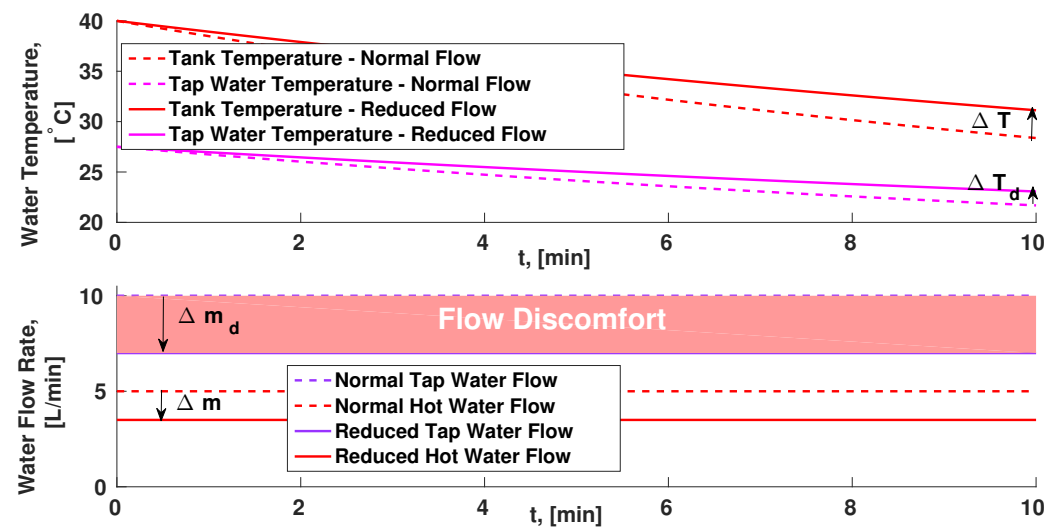

Figure 3.4: Possible flow discomfort zones.

Deviations of the water flow $\dot{m}_{\mathrm{d}}(t)<\dot{m}_{\mathrm{c} \cdot \min }(t)$ and $\dot{m}_{\mathrm{d}}(t)>\dot{m}_{\mathrm{c} \cdot \max }(t)$ can have a different impact on the user, which calls for the necessity to distinguish between them. Furthermore, since the user can, in general, adjust the flow $\dot{m}_{\mathrm{d}}(t)$ multiple times during the WA, the expression in Equation 3.6 can be modified as follows:

$$
A_{\dot{m}_{\mathrm{d}}}(t)=\int_{0}^{t} \max \left\{0, \dot{m}_{\mathrm{c} \cdot \min }(t)-\dot{m}_{\mathrm{d}}(t), \dot{m}_{\mathrm{d}}(t)-\dot{m}_{\mathrm{c} \cdot \max }(t)\right\} d t
$$

The expression in Equation 3.7 represents only the environmental variable $\dot{m}_{\mathrm{d}}(t)$ of the user comfort related to the tap water flow. Since the user satisfaction with the tap water flow is also determined by the personal variables such as the mechanoreceptors, skin sensitivity and thickness and other (Section 3.1.3), Equation 3.7 cannot adequately describe the user flow comfort.

To account for the personal user perception of the water flow variations, we need to regard the personal variables that determine that feeling. In view of a big diversity of such personal variables that can intricate our user flow comfort model, we incorporate the user flow tolerance as a delegate (or representative) of the personal variables. Serving as an abstraction for the personal variables, the user flow tolerance ${ }^{7}$ describes the user ability to tolerate uncomfortable tap water flow rates.

Similar to the user thermal tolerance in Section 3.2, we model the user tolerance to the tap water flow variations by a piece-wise linear function:

$$
F_{\dot{m}_{\mathrm{d}}}\left(\dot{m}_{\mathrm{d}}, t\right)=\left[\begin{array}{ll}
\gamma_{1}(t)\left[\dot{m}_{\mathrm{d}}(t)-\dot{m}_{\mathrm{c} \cdot \min }(t)\right]+\mu_{1}(t) & , \text { if } \dot{m}_{\mathrm{d}}(t) \in \Delta_{\mathrm{tol}}^{-}(t) ; \\
\gamma_{2}(t)\left[\dot{m}_{\mathrm{d}}(t)-\dot{m}_{\mathrm{c} \cdot \max }(t)\right]+\mu_{2}(t) & , \text { if } \dot{m}_{\mathrm{d}}(t) \in \Delta_{\mathrm{tol}}^{+}(t) ; \\
0 & , \text { if } \dot{m}_{\mathrm{d}}(t) \in\left[\dot{m}_{\mathrm{c} . \min }(t), \dot{m}_{\mathrm{c} \cdot \max }(t)\right] ; \\
1 & , \text { otherwise; }
\end{array}\right.
$$

\footnotetext{
${ }^{7}$ The presence of the user tolerance to different water flows can be justified by the existing threshold for the mechanoreceptor activation, as narrated in Section 3.1.3.
} 
where $\gamma_{1}(t) \leq 0, \mu_{1}(t) \geq 0$ and $\gamma_{2}(t) \geq 0, \mu_{2}(t) \geq 0$ are the coefficients that describe the intensity of the user flow comfort degradation; $\Delta_{\text {tol }}^{+}$and $\Delta_{\text {tol }}^{-}$stand for the water flow rates tolerated by the user.

The function $F_{\dot{m}_{\mathrm{d}}}$ in Equation 3.8 shows how the user comfort related to the tap water flow rate is lost in case of a deviation of the received water flow rate $\dot{m}_{\mathrm{d}}(t)$ from the comfort boundaries $\dot{m}_{\text {c.min }}(t)$ or $\dot{m}_{\text {c.max }}(t)$ at time $t$. By analogy to the user thermal tolerance function $F_{\mathrm{T}}$, we require $0<\gamma_{1}(t)\left[\dot{m}_{\mathrm{d}}(t)-\dot{m}_{\mathrm{c} \text {.min }}(t)\right]+\mu_{1}(t)<1$ if $\dot{m}_{\mathrm{d}}(t) \in\left(\dot{m}_{\mathrm{c} \text {.min }}(t)-\right.$ $\left.\Delta_{\text {tol }}^{-}(t), \dot{m}_{\text {c.min }}(t)\right)$ and $\Delta_{\text {tol }}^{-}(t)>0$, while $0<\gamma_{2}(t)\left[\dot{m}_{\mathrm{d}}(t)-\dot{m}_{\mathrm{c} \cdot \max }(t)\right]+\mu_{2}(t)<1$ when $\dot{m}_{\mathrm{d}}(t) \epsilon$ $\left(\dot{m}_{\mathrm{c} . \max }(t), \dot{m}_{\mathrm{c} . \max }(t)+-\Delta_{\text {tol }}^{+}(t)\right)$ and $\Delta_{\text {tol }}^{+}(t)>0$. Besides, the values of the coefficients $\gamma_{1}(t)$ and $\gamma_{1}(t)$ are allowed to be $\gamma_{1}(t)=-\frac{1}{\Delta_{\text {tol }}^{-}(t)}<0$ if $\mu_{1}(t) \geq 0$, or $\gamma_{1}(t)=0$ when $\mu_{1}(t)>0$; and $\gamma_{2}(t)=\frac{1}{\Delta_{\text {tol }}^{+}(t)}>0$ in case $\mu_{2}(t) \geq 0$, or $\gamma_{2}(t)=0$ when only $\mu_{2}(t)>0$.

The tolerance function $F_{\dot{m}_{\mathrm{d}}}$ for the case $\gamma_{1}(t)<0, \mu_{1}(t)=0$ and $\gamma_{2}(t)>0, \mu_{2}(t)=0$ is illustrated in Figure 3.5 at the moment of time $t$.

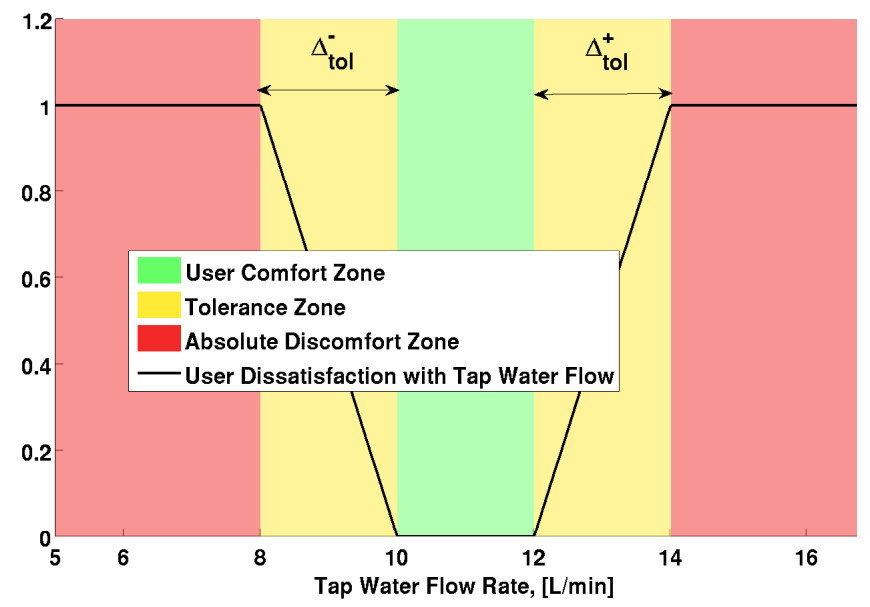

Figure 3.5: User water flow tolerance function $F_{\dot{m}_{\mathrm{d}}}\left(\dot{m}_{\mathrm{d}}, t\right)$ at time $t$.

Since the tolerance function $F_{\dot{m}_{\mathrm{d}}}$ in Equation 3.8 is, in general, a function of time $t$, a person might have different functions (or snapshots) at other moments of time during a single WA. For the purpose of simplicity, we assume hereinafter that the shape of the flow tolerance function does not change during the same WA, i.e. $F_{\dot{m}_{\mathrm{d}}}\left(\dot{m}_{\mathrm{d}}, t\right)=F_{\dot{m}_{\mathrm{d}}}\left(\dot{m}_{\mathrm{d}}\right)$.

The user flow comfort can, however, deteriorate distinctly from one WA to another. Due to a vast diversity of WAs at home, it seems difficult to differentiate between the user flow tolerance per WA. For simplicity, we assume that the flow rate tolerance does not change within a certain category of WAs. Thus, for example, the user may have similar preferences for tap water flow whenever he washes his hands in the tap water. We assume that every $i$-th user has the water flow tolerance functions $F_{\dot{m}_{\mathrm{d}}, i, j}$ each corresponding to a certain category $j$ of the WA. To this extent, each function $F_{\mathrm{T}, i, j}$ incorporates the personal comfort variables in the context of the WA categories.

By combining this contextual information with the expression in Equation 3.7, we 
can consider both the environmental and personal variables of the user flow comfort in a context of the WA categories. The expression $D_{\dot{m}_{d}}(t)_{i, j}$ that characterizes the flow discomfort of the $i$-th user during a single WA belonging to the $j$-th type of WAs can be formalized as follows:

$$
D_{\dot{m}_{d}}(t)_{i, j}=F_{\dot{m}_{\mathrm{d}}, i, j}\left(\dot{m}_{\mathrm{d}}\right) A_{\dot{m}_{\mathrm{d}}}(t)_{i, j}
$$

where $A_{\dot{m}_{\mathrm{d}}}(t)_{i, j}$ is the area between the tap water flow rate $\dot{m}_{\mathrm{d}}(t)$ experienced by the $i$-th user at time $t$ (during a single WA of the $j$-th category) and the lower and(or) upper comfort boundaries $\left[\dot{m}_{\text {c.min }}, \dot{m}_{\text {c.max }}\right]$.

The expression $D_{\dot{m}_{d}}(t)_{i, j}$ in Equation 3.9 accounts for both the user comfort personal and the environmental variables through the terms $F_{\dot{m}_{\mathrm{d}}, i, j}$ and $A_{\dot{m}_{\mathrm{d}}}(t)_{i, j}$, respectively.

Akin the user thermal comfort characteristic in Equation 3.5, the expression $D_{\dot{m}_{d}}(t)_{i, j}$ in Equation 3.9 can serve only as an approximate measure of the real comfort of the user related to the tap water flow.

\subsection{ConcLuSion}

This chapter highlights the necessity of user comfort modeling in case of a modified operation of electric tank water heaters (WHs). In scenarios where a regular deadband control of the WH is modified (e.g., the WH is temporarily shut off by demand response), there is a risk of user comfort deterioration. To be able to control the WH without a breach of user comfort preferences, it is important to consider the impact of control actions on end-user comfort. As a possible way to increase the awareness about user comfort in control of WHs, end-user comfort can be modeled and further accounted for in the control. The chapter gives an insight into how to quantify the end-user satisfaction with the tap water during hot water activities (WAs) and proposes two comfort models in that regard, namely the user thermal comfort and flow comfort models.

While the user thermal comfort model gives a numerical quantification of the user satisfaction with the tap water temperature, the user flow comfort model describes how comfortable the user finds the tap water flow rate when having a direct contact with running water.

Both of the models incorporate the information about the environmental and personal variables that influence the feeling of comfort of an individual. By including the user tolerance to tap water temperature and tap water flow deviations into the models, it makes it possible to determine the real boundaries of the user comfort during WAs. The user tolerance can be further exploited in the control to perform control actions exactly on the boundary of the user comfort, yet providing the hot water service acceptable to the end-user.

The proposed models account for the fact that not only the instantaneous deviations of the environmental parameters such as the tap water temperature and the water flow rate during WAs matter for the user, but also how long the user experiences such deviations. Therefore, the comfort models can be used for numerical estimation of the user comfort experienced during the hot water usage in different time frames (e.g., day or week).

The application of the devised comfort models to control of the WH will be demonstrated in the following chapters. 



\section{4 \\ Maintaining COMFORT FOR Single Water Activity}

Two control strategies to maintain user comfort at the user-desired level are presented and compared to the conventional deadband control of WHs in this chapter. A central idea of the proposed control is to align the amount of heat stored in the WH tank exactly to the comfort level required by the end-user. We argue that storage of only the amount of heat truly needed by users for their hot water activities (WAs) may be more advantageous than storing a fixed and usually overrated amount of heat. The simulation results of the proposed control mechanisms reveal the possibilities to preserve the user thermal comfort while reducing the heat losses and electricity consumption for water heating. In this chapter, we focus on a simplified scenario of the hot water usage considering only a single WA.

$\mathrm{H}$ ot water systems (HWSs) in households use heating units to produce hot water. Residents expect that the tap water is at desired temperatures during preferred periods of a day. Therefore, the purpose of domestic water heaters is to provide hot water in the amounts suitable for the requirements of occupants.

When an end-user receives hot water at the desired temperature, his thermal comfort is at the maximum. However, if the hot water production is too low, users may feel uncomfortable due to too cold water temperature during hot water activities (WAs), i.e. they may experience thermal discomfort. Such a mismatch between hot water production and consumption may appear due to the technical limitations of water heaters and(or) an intense hot water demand during WAs resulting from high hot water flow rates/temperatures or long-lasting, simultaneous or frequent hot water usage.

The two most popular configurations of water heaters in European countries are instantaneous (tankless) water heaters and water heaters connected to a tank to maximize their efficiency [18]. Heaters of the first type heat the water directly when it flows through the heating unit and thus typically have high heating capacities. In contrast, tank water heaters (WHs) can use less heating power as they can prepare hot water in their storage tanks upfront the usage. Since the power demand of WHs is typically lower than that of 
instantaneous heaters, there is a risk that the heat available in the tank may be inadequate to cover hot water demand, especially in case of intense WAs.

In general, the heating power of $\mathrm{WH}$ is insufficient for heating the flowing water without a backup of a tank during residential hot water usage. As a result, the tank water temperature decreases during intense WAs, causing the drop of the tap water temperature and finally leading to the user thermal discomfort. As such, the question of fulfilling the user thermal comfort boils down to a question of having sufficient thermal energy in the tank during WAs.

However, the most straightforward strategy to always heat up the tank to the maximum allowed temperature is, in general, not the best solution from economical and sustainability perspectives. If the heat available in the tank exceeds the real heat demand in the next hours, the stored heat will be dissipated to the environment in the form of heat losses, which leads to a waste of energy and money. To reduce unwanted heat losses, it would be ideal to heat the water in the tank as late as possible and only up to the temperature required by the expected WA. To sum up, for an efficient operation of WHs, a compromise between fulfilling the user requests and losses of the valuable heat should be found. This problem can be formulated as maximizing the user thermal comfort while minimizing the heat losses during the $\mathrm{WH}$ operation.

If we consider the existing thermostat deadband control of WHs, we note the following limitations that do not allow to efficiently maintain the user thermal comfort:

- No information about the future WAs and user comfort is utilized in control.

- The lower and upper thermostat setpoint temperatures are fixed.

- Raising thermostat setpoint temperatures in the deadband control may improve the user thermal comfort, but results in greater heat losses.

- There is a risk that the tank water temperature is too low at the beginning of the WA so that the user will experience the thermal discomfort.

To overcome the mentioned problems, the WH control mechanism should be capable to adjust hot water production to the expected user comfort needs. Residential hot water consumption is characterized by a vast variety of possible WAs. Such WAs are stochastic and so are the user thermal comfort requirements associated with them. As the comfort needs may vary for different users as well as for a single user during a day, the new control mechanism should be based on a forecast of WAs including their user comfort requirements.

In this chapter, we propose two control mechanisms that allow for the hot water provision in compliance with the user-desired comfort while minimizing the heat losses from the WH tank, namely the pre-heating control and the flow control. The first control algorithm, the pre-heating control, is aimed at providing the user with a tap water temperature within a range of comfortable temperatures during a WA. In other scenarios of residential hot water consumption, a stable tap water temperature may be desirable (e.g., showering). We therefore introduce the second control algorithm, the flow control, suitable to supply a nearly stable tap water temperature during a WA. Both the mechanisms act on a continuous-time planning horizon. 
We assume that information about the upcoming hot water consumption and the desired user comfort related to it are known from an ideal forecast ${ }^{1}$. For simplicity, we consider a case of a single WA in this chapter. The considered HWS consists of a WH, a single tapping point with a mixer tap and a system of pipes that connect the components with a cold water mains of a household. The tapping point accommodates a specific mixer tap that allows the user to only turn hot and cold water on and off and regulate the tap water flow rate, as opposed to traditional (monobloc) mixer taps that allow the user to also regulate the temperature. That is to say, we make an assumption that the user does not adjust the ratio of the hot and cold water flows in the mixer tap. Instead, the water flows are adjusted automatically with respect to the user-desired tap water temperature (range) by means of a mixer tap controller. The tank water temperature is regulated by means of a controller of the heating elements of the HW.

The proposed control mechanisms are build upon two comfort models (presented in Chapter 3): to quantify user comfort preferences:

- The thermal comfort model that reflects user satisfaction with the tap water temperature.

- The flow comfort model that estimates user satisfaction with the tap water flow rate.

To account for the thermodynamics of the WH in our control, we incorporate a well-mixed WH tank model presented in Section 2.5.2.

First, we focus on a scenario where the end-user does not require a stable tap water temperature during the WA, i.e. he is able to tolerate the deviation of the tap water temperature from its initial value (at the beginning of the WA) in a certain range. From the thermodynamic theory, it is known that there exist multiple combinations of the tank water temperature and water flow rates that result in the same tap water temperature in the considered HWS. The idea of the pre-heating control introduced in Section 4.4 is to establish the best tank water temperature and the best combination of hot and cold water flows at the beginning of a WA that maximally satisfy the user-desired thermal comfort and minimize the heat losses. In the pre-heating control, the all the water flows in the mixer tap are fixed during the WA. The control influences the tap water temperature during the WA, i.e. the user thermal comfort, by means of three control variables: (i) the tank water temperature, (ii) the flow rate of hot water from the $\mathrm{WH}$ and (iii) the cold water flow rate from the mains, as illustrated in Figure 4.1.

\footnotetext{
${ }^{1}$ An ideal forecast contains no errors.
} 


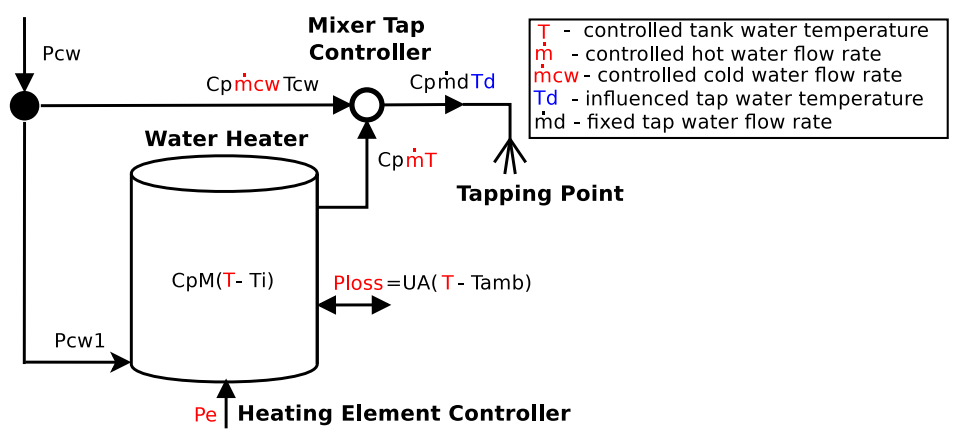

Figure 4.1: Parameters of the pre-heating control.

To be specific, we assume that the user does not adjust himself the ratio of the hot and cold water flows in the mixer tap, but only sets the preferred tap water flow rate at the beginning of a WA. Since this tap water flow rate is fixed during the WA and known from the forecast, we only have to find the best combination of two control variables, i.e. the tank water temperature and the hot water flow rate, at the start time of the WA, while the cold water flow can be found by subtracting the hot water flow rate from the known tap water flow rate. To find the best combination of these two control variables in terms of the user thermal comfort and the heat losses, we deal with simultaneous maximization of the user thermal comfort and minimization of the heat losses during a WA. We apply a multi-objective optimization approach to solve this problem in Section 4.4.2.

The pre-heating control is implemented as follows. The controller of the WH tracks the current tank water temperature value and initiates the water heating upfront the WA so that the found tank water temperature is established exactly at the WA startup. Once the tank water temperature is established the WH remains off for the rest of the time. When the user opens the tap and sets his preferred tap water flow, the hot and cold water flow rates are set to the found values by the mixer tap controller.

Next, we concentrate on a scenario of hot water usage where a stable tap water temperature is required. There exist different engineering solutions such as thermostatic mixing valves (TMVs), pressure valves, digital taps, etc. that can fulfill this task. In one of the most popular solutions, TMVs, thermosensitive element can react to variations of the tap water temperature and stabilize it by adjusting the hot and cold water inflows. An apparent advantage of TMVs is that they regulate the tap water temperature automatically based on the physical properties of the thermosensitive material. One of the shortcomings of TMVs is that their control is decoupled from the water heating control on the side of the $\mathrm{WH}$. That is to say, the water heating mechanism and the flow control work independently from each other, i.e. the water is heated without regard to the real temperatures required for TMV to provide the user-desired stable tap water temperature. Such an operation is not optimal from the economical and user comfort points of view because it can lead to overheated or underheated tank water at the beginning of the WA. In case the tank water is too cold, TMV cannot support the user-desired stable tap water temperature during the WA. Furthermore, if the WH tank is overheated, the heat will not be utilized efficiently during the WA and its residuals will be wasted for the heat losses. A more detailed discussion of pros and cons of the standard solutions can be found in Section 4.5.1. 
To be able to provide a stable tap water temperature with awareness of the water temperature in the WH tank (increasing or decreasing it before the WA when necessarily), we introduce the second control mechanism by combining a similar to TMVs flow control with the pre-heating control. As in TMVs, the idea is to regulate the water hot and cold water flow rates with the progress of a WA in order to maintain a nearly stable userdesired tap water temperature. As contrasted to TMVs, the flow control algorithm allows for finding the best tank water temperature values to be established by the pre-heating component of the mechanism. The flow control component standalone influences the tap water temperature by controlling two variables with the progress of a WA: (i) the flow rate of hot water coming from the WH tank and (ii) the flow rate of cold water, as shown in Figure 4.2 .

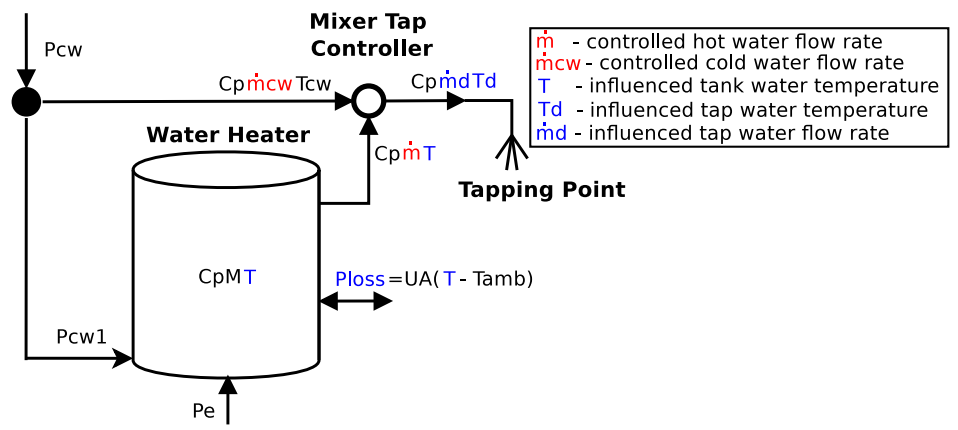

Figure 4.2: Parameters of the flow control component (standalone operation).

The combined control mechanism additionally controls the tank water temperature at the WA startup are depicted in Figure 4.3.

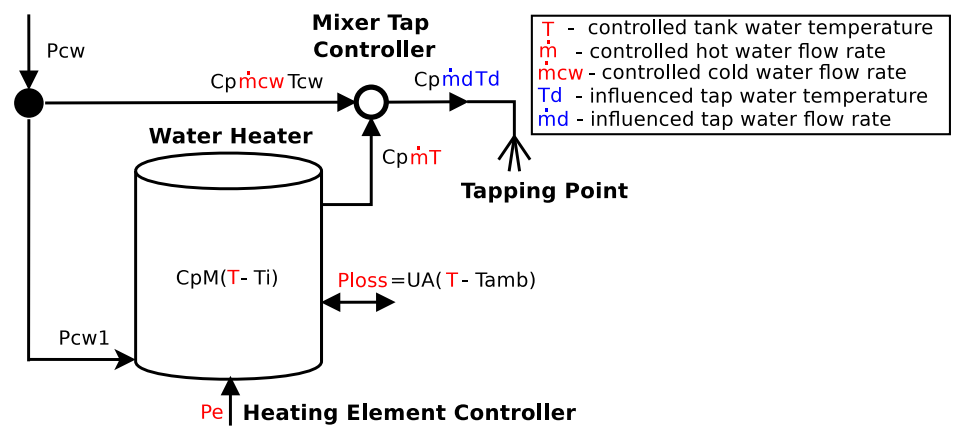

Figure 4.3: Parameters of the combined pre-heating and flow control.

Similar to the previous scenario of hot water usage, the WH is turned off during a WA. Unlike the previous scenario where the hot and cold water flow rates are set only once (when the tap is open) and further remain fixed, the water flows can be varied during the WA by the flow control. Furthermore, in the current scenario we relax the condition that the tap water flow rate is fixed during a WA, i.e. the tap water flow can vary during the WA. To fulfill the user requirement for a stable tap water temperature during a WA, the tank 
water temperature should be at least equal to the required tap water temperature value by the end of the WA. We can exploit this condition and find the best combinations of the water flows working backwards from the end time of the WA. By breaking down the WA timeline into even steps, we apply a dynamic programming optimization approach to solve this problem. The objectives pursued by the optimization are (i) the user-desired tap water temperature value at the beginning of each step, (ii) the minimization of the user flow discomfort that can arise due to the varied tap water flow rate and (iii) the minimization of the heat losses during the WA so that the heat is efficiently utilized during the WA. The solution to the problem is a triplet $\left\{\dot{m}, \dot{m}_{\mathrm{cw}}, T\right\}_{i}$ at every $i$-th step of the flow control. Thus, the tank water temperature value $T_{1}$ at the beginning of the first step (the WA start time) is the best tank water temperature value that provides a stable tap water temperature for a given WA under the miniminum user flow discomfort and minimum heat losses during the WA. This temperature value can be established by heating the water prior to the WA as in case of the pre-heating control. In this way, the combined pre-heating control and flow control mechanism allows to both create the best amount of heat in the WH tank and utilize it efficiently in a scenario of a nearly stable tap water temperature.

Summarizing, the control mechanisms proposed in this chapter are capable to serve hot water production with high respect to the end-user comfort while minimizing the heat losses in scenarios without and with the requirement for a stable tap water temperature.

\subsection{User Discomfort in Deadband Control}

First and foremost, the hot water temperature inexorably drops in the considered system in Figure 2.1. When the mixer tap is closed, the thermal energy stored in the WH tank dissipates to the environment through the jacket of the $\mathrm{WH}$, the so-called stand-by losses. Whereas during the hot water usage the tank water temperature falls mainly due to the inflow of cold water in the tank. Since the thermostat setpoints of the WH are set to pre-defined values and typically are seldom adjusted by users, the hot water demand may cause a substantial drop of the tank water temperature and consequently of the tap water temperature. In case the tap water temperature is too low, the user may experience thermal discomfort.

As discussed in Section 2.1, the heating capacities of WHs are often insufficient to provide a stable tap water temperature which may be desireable during some WAs (e.g., showering). Even relatively small hot water flows during WAs can lead to the tank water temperature decrease (as it will be shown later in this chapter) and cause user thermal discomfort.

\subsection{Organization of Chapter}

The central problem considered in the following is a problem of preserving the user thermal comfort during a single hot water water activity (WA) in the HWS shown in Figure 2.1. In this chapter, we deal with control strategies for mitigation of possible disruptions of the user thermal comfort, rather than with engineering solutions (e.g., improved insulation).

We focus on two distinct scenarios of the residential hot water usage:

- a scenario where the user can tolerate a range of tap water temperatures; 
- a scenario where the user desires a stable tap water temperature.

In that regard, we consider two control strategies. To ensure the user thermal comfort, one of the strategies is to pre-store a sufficient amount of heat in the WH tank upfront a WA. However, even after pre-heating the water up to the maximum safety allowed temperature $\left(90^{\circ} \mathrm{C}\right)$, the user will not receive a stable tap water temperature during the WA, because the $\mathrm{WH}$ is incapable to heat the running water and there is no thermostatic element in the mixer tap (i.e. no TMV). This situation calls for an additional control mechanism which has a goal to evenly dispense the stored thermal energy during the WA.

Concerning the water pre-heating strategy, it is crucial to know: (i) when to start the water heating, (ii) how to withdraw the hot water from the WH tank so that the tap water temperature is within the user comfortable range or results in a thermal comfort acceptable for the user during a WA. The second control strategy requires management of the hot water withdrawal from the WH during a WA. Therefore, the two different control problems tackled in this chapter can be, in general, expressed as:

1. scheduling of the WH heating periods and the hot water withdrawal to provide the tap water in a user-desired range of temperatures;

2. management of hot water withdrawal to maintain a stable tap water temperature.

A more precise formulation of the above problems is given in the next chapters.

\subsection{Water Pre-heating Strategy}

To begin with a control strategy to support a scenario where the user can tolerate a range of tap water temperatures, we briefly provide some background information.

An idea to pre-heat the $\mathrm{WH}$ to a higher temperature than normal operative setpoints $\left(50-70^{\circ} \mathrm{C}\right)$ is not new and can be found in such demand side management applications as reduction of the peak electricity demand [67] and reduction of water heating costs [66]. For example, the thermostat upper setpoint temperature can be raised upfront the period of high electricity prices during which the WH is allowed to coast without electricity consumption [66]. As a result of shifting of the $\mathrm{WH}$ load outside the high-price period, the water heating costs can be reduced. It is apparent that the new setpoint temperature should be chosen with respect to a possible hot water consumption during the WH disconnection period. In case the new setpoint is too low, there is a risk that users can receive unsatisfactory cold water. In contrast, if the tank water temperature is too high and there is no hot water usage expected during the disconnection period, the pre-stored heat will be wasted for the heat losses.

Inspired by the pre-heating strategy in $[66,67]$, in order to mitigate a possible user thermal discomfort we propose to modify the deadband control of a WH by increasing the amount of heat stored in the WH tank above the usual setpoints prior to a WA.

\subsubsection{Pre-heating Problem}

Let a single hot water activity (WA) occur at time $t_{\mathrm{WA}}$ on a continuous timescale of a day. Suppose that the user desires to maximally satisfy his thermal comfort requirement during the WA. 
If the initial tank water temperature is insufficient to fulfill the user comfort request, extra heat can be pre-stored. A controller of the $\mathrm{WH}$ can initiate water heating prior to the WA, for example, as illustrated in Figure 4.4(a). The pre-heating can be accomplished in different ways depending on the initial and final tank water temperature values as depicted in Figure 4.4(b). First, suppose the user thermal comfort can be satisfied, if the tank water temperature at the beginning of the WA is equal to $90^{\circ} \mathrm{C}$. As it can be seen from Figure 4.4(b), in the worst-case scenario where the $\mathrm{WH}$ is initially filled with cold water $\left(T(0)=15^{\circ} \mathrm{C}\right)$, the pre-heating procedure may take up to $2.4[\mathrm{~h}]$. However, in case the user comfort can be satisfied under the tank water temperature equal to $65^{\circ} \mathrm{C}$ (case II in Figure 4.4(b)), and the initial temperature of water inside the tank is equal to $70^{\circ} \mathrm{C}$, there is no need to heat up the water at all.

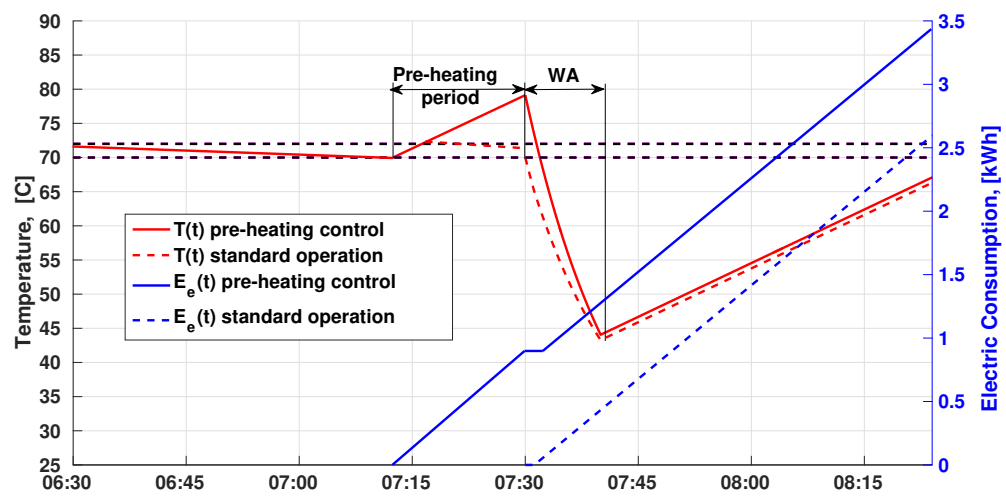

(a)

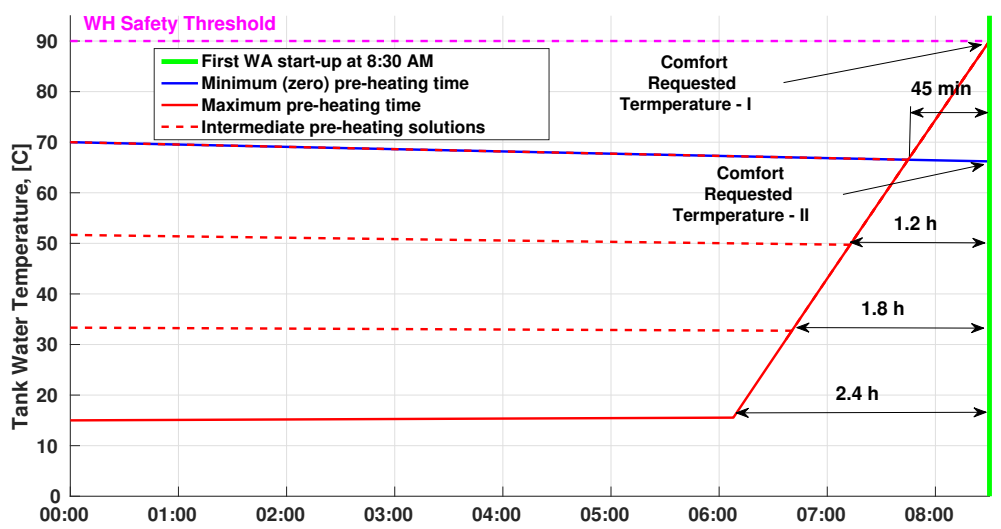

(b)

Figure 4.4: (a) Idea of water pre-heating, (b) possibilities to pre-heat the WH.

If the required comfort level is relatively high, the user may end up with a higher electricity consumption than in the conventional deadband control. Because the increased electricity expenses may potentially outweigh the user desire to satisfy his thermal comfort, and vice versa (the user may be more interested in his thermal comfort than in the consumed 
electricity), the user may then decide in favor of either the minimization of the electricity consumption or minimization of the thermal comfort disruptions. These two objectives are in conflict with each other during the WA.

Not only depends the user comfort satisfaction during a WA on the tank water temperature at the WA startup, but also on the way the pre-stored heat is withdrawn from the WH tank during the WA, i.e. it depends on the hot water outflow from the WH tank. It is known from the thermodynamic theory that there can exist multiple combinations of tank water temperature values and hot water flow rates that result in the same value of the tap water temperature. For instance, a feasible set of tank water temperature and hot flow rate values that provide the same momentary tap water temperature value equal to $40^{\circ} \mathrm{C}$ under the tap water flow rate $\dot{m}_{\mathrm{d}}(t)=10[\mathrm{~L} / \mathrm{min}]$ is represented by a relationship in Figure 4.5 .

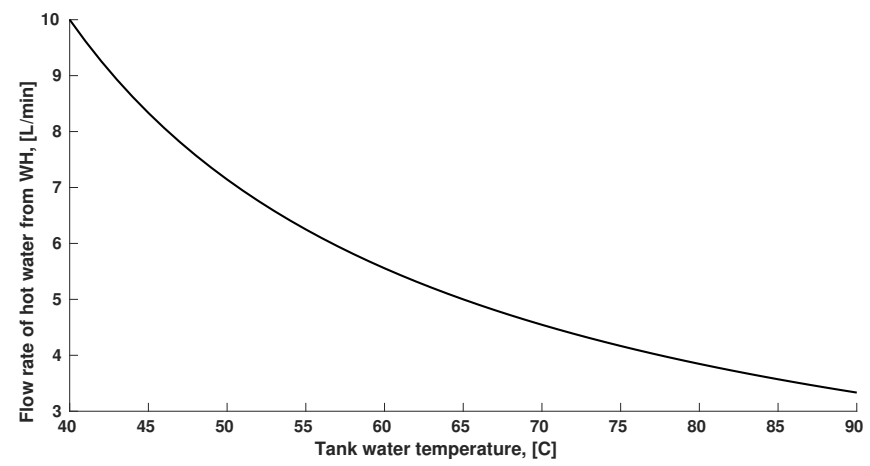

Figure 4.5: Multiple solutions that result in tap water temperature $T_{\mathrm{d}}(t)=40^{\circ} \mathrm{C}$ at moment $t$.

One can notice from Figure 4.5 that lower temperature values $T(t)\left(\right.$ e.g. $\left.40^{\circ} \mathrm{C}\right)$ at time $t$ are more beneficial in terms of the electricity consumption, however, there is no guarantee that such values will meet user thermal comfort requirement during the entire time of the $\mathrm{WA}^{2}$. Therefore, the task is to find the best combination of the tank water temperature and the hot water flow rate at the start of the WA that provide the best satisfaction of the user thermal comfort during the entire WA. That best tank water temperature value should be then established by the water pre-heating mechanism.

The pre-heating problem can be formulated as follows.

\section{PRE-HEATING PROBLEM}

Find the best option(s) for heating the water and for its delivery

that minimize the user thermal discomfort during a single WA while minimizing the electricity consumption for water pre-heating.

\footnotetext{
${ }^{2}$ The plot only corresponds to a momentary value $T_{\mathrm{d}}(t)=40^{\circ} \mathrm{C}$ at time $t$.
} 


\subsubsection{Pre-heating Problem Clarification}

In our approach, we utilize the user thermal comfort model expressed by Equation 3.5 to quantify user satisfaction with the tap water temperature during a given WA. Since the user thermal discomfort $D_{T}(t)$ is a function of the tap water temperature $T_{\mathrm{d}}(t)$ and the temperature $T_{\mathrm{d}}(t)$ is determined by the the water temperature $T(t)$ inside the tank and the outflow from the tank $\dot{m}(t)$ as in Equation 2.7, we can conclude that two variables that should be controlled to fulfill the user thermal comfort are $T(t)$ and $\dot{m}(t)$.

Consider a single 10-minute WA with the user desired tap water flow $\dot{m}_{\mathrm{d}}=10[\mathrm{~L} / \mathrm{min}]$ and preferred temperature $T_{\mathrm{d}}$ in the range $[40,45]^{\circ} \mathrm{C}$. Suppose that the temperature $T_{\mathrm{d}}$ can be established at the beginning of the WA $t_{\mathrm{WA}}$ by a controller located at the tapping point. Whereas another controller located at the WH can perform the water pre-heating before $t_{\mathrm{WA}}$. Furthermore, assume that the WH is switched off during the WA.

Various combinations of the tank water temperature $T\left(t_{\mathrm{WA}}\right)$ and the hot water flow rate $\dot{m}\left(t_{\mathrm{WA}}\right)$ at the beginning of the WA $t_{\mathrm{WA}}$ determine a domain of the tap water temperature $T_{\mathrm{d}}(T, \dot{m})$ function in Equation 2.7. The contours in Figure 4.6(a) refer to possible outcomes of the temperature $T_{\mathrm{d}}\left(t_{\mathrm{WA}}\right)$ under different combinations $\left\{T\left(t_{\mathrm{WA}}\right), \dot{m}\left(t_{\mathrm{WA}}\right)\right\}$.

Then, the region between the curves $T_{\mathrm{d}}\left(t_{\mathrm{WA}}\right)=40^{\circ} \mathrm{C}$ and $T_{\mathrm{d}}\left(t_{\mathrm{WA}}\right)=45^{\circ} \mathrm{C}$ reflects multiple solutions $\left\{T\left(t_{\mathrm{WA}}\right), \dot{m}\left(t_{\mathrm{WA}}\right)\right\}$ that provide a the temperature $T_{\mathrm{d}}\left(t_{\mathrm{WA}}\right)$ in the user-desired range $[40,45]^{\circ} \mathrm{C}$, as indicated by the green region in Figure 4.6(a).

Noteworthy, two subsets of the solutions $(\mathrm{a})$ when $T\left(t_{\mathrm{WA}}\right)=40^{\circ} \mathrm{C}$ and $(\mathrm{b})$ when $T\left(t_{\mathrm{WA}}\right)=$ $90^{\circ} \mathrm{C}$ correspond to different levels of the electricity consumption for water pre-heating, i.e. $E_{e, \text { min }}=P_{e} \Delta t\left(T_{\mathrm{cw}}, 40^{\circ} \mathrm{C}\right)<E_{e, \text { max }}=P_{e} \Delta t\left(T_{\mathrm{cw}}, 90^{\circ} \mathrm{C}\right)$, where $t\left(T_{\mathrm{cw}}, T\left(t_{\mathrm{WA}}\right)\right)$ refers to the time needed to heat all the tank water from $\left(T_{\mathrm{cW}}\right.$ to $T\left(t_{\mathrm{WA}}\right)$ (Equation 2.3). Thus, the best solution in terms of the electricity consumption for this WA is $E_{e, \min }$. However, the value $E_{e}=E_{e, \min }$ does not imply the maximum user comfort during the WA as can be seen in Figure 4.6(b). More specifically, the solution $\left\{T\left(t_{\mathrm{WA}}\right), \dot{m}\left(t_{\mathrm{WA}}\right)\right\}=\left\{40^{\circ} \mathrm{C}, 10[\mathrm{~L} / \mathrm{min}]\right\}$ causes higher thermal discomfort than in case of the $\left\{T\left(t_{\mathrm{WA}}\right)=90^{\circ} \mathrm{C}, \dot{m}\left(t_{\mathrm{WA}}\right)\right\}$. This can be explained by the fact that the higher outflow $\dot{m}\left(t_{\mathrm{WA}}\right)$ leads to a faster discharge of the $\mathrm{WH}$. This also means that a trade-off between the conflicting objectives of the minimization of the user thermal discomfort and minimization of the electricity consumption should be found.

In other words, we need to find such optimal temperature(s) $T^{*}$ in the tank at the beginning of the WA and hot water flow rate(s) $\dot{m}^{*}$ during the WA that minimize user thermal discomfort $D_{\mathrm{T}}$ while minimizing energy consumption for pre-heating $E_{e}$, provided that all parameters of the WA and comfort model parameters are known (e.g., from an ideal forecast). The tank water temperature $T\left(t_{\mathrm{WA}}\right)$ and the hot water flow rate $\dot{m}$ to be established are the control variables in the current problem.

\subsubsection{Assumptions}

We imply the following assumptions for the pre-heating problem:

- a single hot water activity (WA) occurs on a planning horizon of a day;

- all the time and thermal comfort related parameters of the WA are known in advance from an ideal forecast; 


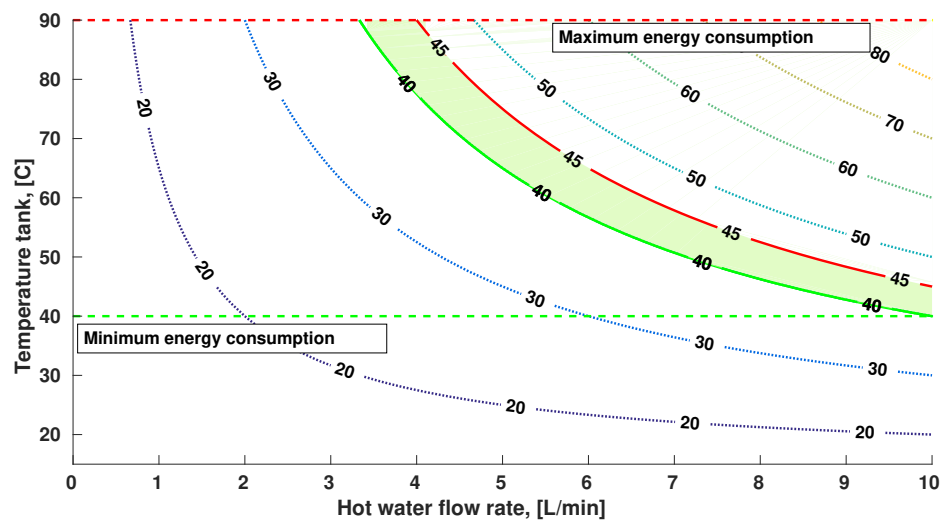

(a)

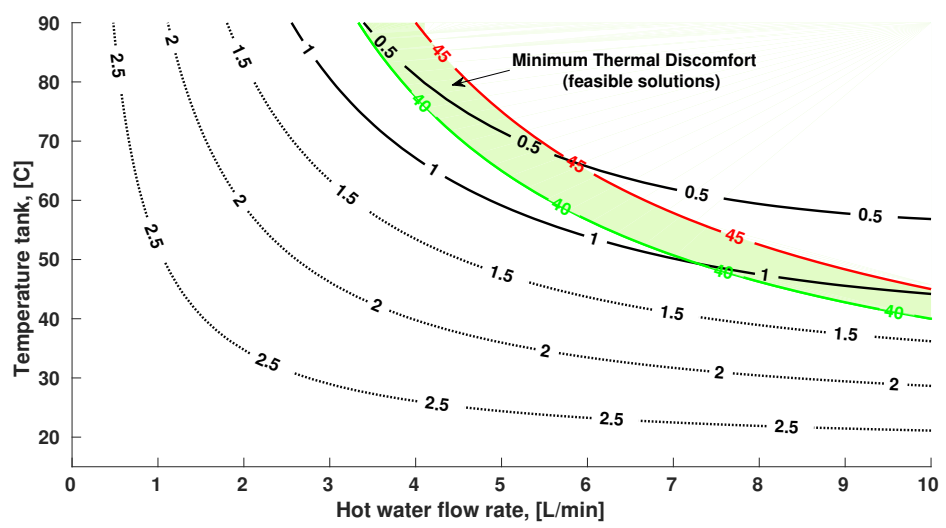

(b)

Figure 4.6: Feasible solutions (a) tap water temperature (b) user thermal comfort.

- the mixer tap is equipped with a flow controller that automatically establishes the hot water flow rate $\dot{m}$ and cold water water flow rate $\dot{m}_{\mathrm{cw}}$ only once at the beginning of the WA, i.e. the user does not adjust the hot and cold water flows himself;

- the user sets the desired tap water flow rate $\dot{m}_{\mathrm{d}}$ at the WA startup, $\dot{m}_{\mathrm{d}}$ is assumed to be fixed during the WA;

- the tank water temperature is established by means of the controller of the heating elements upfront the WA, the WH is then swithed off during the WA;

- the maximum allowed tank water temperature $T_{\text {wh, max }}$ should never exceed $90^{\circ} \mathrm{C}[109]$ for safety reasons ${ }^{3}$;

- the ambient temperature $T_{\mathrm{amb}}$ and the cold water tempeature $T_{\mathrm{cw}}$ are fixed during a day.

${ }^{3}$ Higher temperatures rapidly build up the pressure in the WH tank because of the water thermal expansion and vapor, which can destroy the walls of the WH tank. 


\subsection{Proposed Pre-heating Control}

Unlike [66, 67], we treat the pre-heating strategy as a measure for comfort preserving in the WH operation rather than an action for shifting the WH electrical load. Due to this, the proposed pre-heating approach is applied to standalone hot water water activities (WAs) each with its own thermal comfort requirement, and not to the periods of peak system demand ([67]) or to periods of hight electricity prices ([66]). In contrast to [66, 67], the suggested control takes into account the thermal dynamics of an individual WH instead of operating with estimates of engineering parameters of multiple WHs. Another specifics is that we consider a WHS with a tap mixer.

Similar to [66], the proposed approach modifies the thermostat setpoints exactly before the hot water usage without searching for an optimal schedule to turn on/off the WH as it was done in [67]. The pre-heating start time is chosen with respect to the stand-by heat losses and the time needed to heat up all the water to the new setpoint temperature. The new setpoints are chosen optimally in the range $\left[T_{\mathrm{cw}}, T_{\mathrm{wh}, \max }\right]$ (not fixed as in [66]) based on the required user thermal comfort. To obtain the optimal tank water temperature, the devised user thermal comfort model in Equation 3.5 is directly incorporated in the pre-heating mechanism and the engineering constraints of the $\mathrm{WH}$ are taken into account.

\subsubsection{Problem Constraints}

Based on assumption (4.3.3), the tank water temperature $T(t)$ should always satisfy the following constraint:

$$
T_{\mathrm{cw}} \leq T \leq T_{\mathrm{wh}, \max },
$$

where $T_{\mathrm{cw}}$ is the cold water temperature; $T_{\mathrm{wh}, \max }$ stands for the maximum allowed temperature in the tank.

Furthermore, a similar box-constraint for the second decision variable, i.e. the hot water flow rate $\dot{m}$, is required during the WA:

$$
0 \leq \dot{m} \leq \dot{m}_{\mathrm{d}}
$$

This constraint means that a non-negative water flow rate $\dot{m}$ cannot be greater than the requested tap water flow rate $\dot{m}_{\mathrm{d}}$.

The tap water temperature $T_{\mathrm{d}}\left(t_{\mathrm{WA}}\right)$ (Equation 2.7) at the beginning of the WA $t_{\mathrm{WA}}$ should belong to a given user comfort zone so that the user does not immediately experience inconvenient temperature:

$$
T_{\text {c.cmin }} \leq T_{\mathrm{d}}\left(t_{\mathrm{WA}}\right) \leq T_{\text {c.max }}
$$

where $T_{\text {c.min }}$ and $T_{\text {c.max }}$ are the minimum and the maximum comfortable tap water temperatures, respectively.

\subsubsection{ObJective Function}

The pre-heating problem is two-fold. On the one hand, we aim at minimization of the user thermal discomfort during a WA. On the other hand, we want to minimize the electricity consumption for water pre-heating.

We employ a continuous time model on a planning horizon of one day in our approach. In general, the tank water temperature at the beginning of the planning horizon $T(0)$ can 
be any temperature in the range $\in\left[T_{\mathrm{cw}}, T_{\mathrm{wh}, \max }\right]$. For convenience, we translate the objective of minimizing the electricity consumption into the objective of minimizing the heat losses during the WA. As it follows from Equation 2.9, the lower the tank water temperature is (or the lesser the electricity consumption for water pre-heating is), the lower is the rate of the heat losses. By limiting the heat losses, we thereby restrict the amount of energy consumed during the pre-heating $E_{e}\left(T_{\mathrm{cw}}, T\left(t_{\mathrm{WA}}\right)\right)$ and subsequently the thermal energy $E_{d}(t)$ that can be delivered to the end-user during a WA. Thus, reduction of $Q_{\text {loss }}$ may lead to the thermal discomfort $D_{T}$ for the user, while the increase of $Q_{\text {loss }}$ results in higher operational temperatures $T$ and higher electricity consumption $E_{e}$.

We treat the objectives of minimizing the user thermal discomfort $D_{T}$ and minimizing the heat losses $Q_{\text {loss }}$ during the WA as two conflicting goals. A multi-objective optimization approach is utilized to solve the above two objectives simultaneously. The optimization problem can be formalized as follows:

$$
\min \left[\gamma D_{T}+(1-\gamma) Q_{\text {loss }}\right],
$$

s.t. the constraints (5.5) - (5.7) in Section 4.4.1,

where $\gamma \in(0,1]$ is a weight coefficient that signifies the importance of the thermal comfort satisfaction.

The first term of the cost function in Equation 4.4 respects the goal of the minimization of the user thermal discomfort, while the second term concerns the goal of the minimization of the heat losses during a WA and consequently minimization of the electricity consumption for water pre-heating. Based on the importance $\gamma$ to fulfill the user thermal comfort, the optimization solver gives more weight to either of two objectives in Equation 4.4. By setting $\gamma>0$, we prohibit an extreme case of the maximum possible thermal discomfort, i.e. when the tank water is cold $T\left(t_{\mathrm{WA}}\right)=T_{\mathrm{cw}}$. The solution of the optimization problem in Equation 4.4 is a pair (or pairs) of values $\left\{T^{*}\left(t_{\mathrm{WA}}\right), \dot{m}^{*}\right\}$ that is the best combination of the tank water temperature value $T^{*}\left(t_{\mathrm{WA}}\right)$ at the WA startup $t_{\mathrm{WA}}$ and the hot water flow rate $\dot{m}^{*}$ during the WA in terms of the user-desired thermal comfort level and the heat losses.

Once $T^{*}\left(t_{\mathrm{WA}}\right)$ is found, we can calculate the best time $t_{\mathrm{pre}, \text { str }}$ to start the water pre-heating. To avoid unnecessary heat losses, the water should be heated as late as possible before the WA so that the tank water temperature reaches the value $T^{*}\left(t_{\mathrm{WA}}\right)$ exactly at the WA startup. The best start time $t_{\text {pre str }}$ can be found by solving the following system of equations that is based on Equation 2.2 and Equation 2.3:

$$
\left[\begin{array}{l}
T\left(t_{\mathrm{pre}, \mathrm{str}}\right)=\left(\frac{\alpha_{0}}{\beta_{0}}+T(0)\right) e^{\beta_{0}\left(t_{\mathrm{WA}}-\Delta t_{\mathrm{pre}}\right)}-\frac{\alpha_{0}}{\beta_{0}} \\
\Delta t_{\mathrm{pre}}=t_{\mathrm{WA}}-t_{\mathrm{pre}, \mathrm{str}}=\ln \left[\frac{\frac{\alpha_{1}}{\beta_{0}}+T^{*}\left(t_{\mathrm{WA}}\right)}{\frac{\alpha_{1}}{\beta_{0}}+T\left(t_{\mathrm{pre}, \mathrm{str}}\right)}\right] \\
\alpha_{0}=\frac{U A T_{\mathrm{amb}}}{M C_{p}} ; \\
\beta_{0}=-\frac{U A}{M C_{p}} \\
\alpha_{1}=\frac{P_{\mathrm{e}}+U A T_{\mathrm{amb}}}{M C_{p}}
\end{array}\right.
$$

where $T\left(t_{\text {prestr }}\right)$ denotes the tank water temperature in the tank at the moment $t_{\text {pre, str }}$ when preheating starts; $T(0)$ is the initial tank water temperature at the beginning of the planning horizon $t=0$. 


\subsubsection{Performance Evaluation}

We solve the problem formulated in Equation 4.4 by changing the weight $\gamma$ in the range $(0,1]$, i.e. we alter the importance of both of the terms $D_{T}$ and $Q_{\text {loss }}$ and find combinations $\left\{T^{*}\left(t_{\mathrm{WA}}\right), \dot{m}^{*}\right\}$ of the decision variables that minimize their weighted sum.

Although water in the tank can be initially at any temperature $T(0) \in\left[T_{\mathrm{cw}}, T_{\mathrm{wh}, \max }\right]$ depending on the previous history of hot water usage and control actions, we assume for simplicity that at the beginning of the planning period $t=0$ the $\mathrm{WH}$ tank is filled with cold water $T(0)=T_{\mathrm{cw}}$ which corresponds to the worst-case scenario when maximum electricity can be consumed by the pre-heating control. We compare the simulation results of the water pre-heating control with the results of the deadband control. The initial conditions for two types of control differ in that the tank water temperature in case of the deadband control $T_{\text {reg.oper. }}(0)=70^{\circ} \mathrm{C}$ at the beginning of the planning period $t=0$, as contrasted to the initial temperature $T(0)=T_{\mathrm{cw}}=15^{\circ} \mathrm{C}$ in case of the pre-heating control. Besides, when comparing the results of simulations under the different initial temperatures $T_{\text {reg.oper. }}(0)>T(0)$, we do not account for $5.14 \mathrm{kWh}$ electricity spent on initial water heating from $T_{\mathrm{cw}}$ to $70^{\circ} \mathrm{C}$ in case of the deadband control. This "virtual" energy delta can be used for a sanitary heating cycle to prevent bacteria Legionella inside the WH tank in case the pre-heating control. We thus solve the pre-heating problem on two different planning periods: (a) restricted by the end of the recovery period in the deadband control WA $t \in\left[0, t_{\mathrm{WA}, \mathrm{rec}}\right]$, (b) restricted by the end of a day $t \in[0,24][\mathrm{h}]$. In most of our simulations, we apply the heat loss coefficient $\mathrm{UA}=0.95$, unless a different coefficient is specifically mentioned.

Because the pre-heating problem is in essence a reconciliation of two conflicting objectives, we have first explored the possibility to attain trade-offs between the goals under varied values of $\gamma$.

Next, we estimated the efficiency of the pre-heating control in comparison with the deadband control under WAs with the varied tap water flow rates $\dot{m}_{\mathrm{d}}$ and durations $\Delta t_{\mathrm{WA}}$.

\section{Pareto Fronts}

First, we obtain Pareto front of solutions represented by pairs of decision variables $\left\{T^{*}, \dot{m}^{*}\right\}_{i}$. Pareto front is defined by solutions that do not dominate over other solutions on the frontier, meaning that any infinitesimal change in any of variables $T$ or $\dot{m}$ leads to either the improvement of the comfort $D_{T} \downarrow$ and increase of the consumption $E_{e} \uparrow$, or reduction of the consumption $E_{e} \downarrow$ and degradation of the comfort $D_{T} \uparrow$. As such, solutions on Pareto front represent the target trade-offs between two conflicting goals. Pareto fronts for several WAs are shown in Figure 4.7 and Figure 4.8. Each of the solutions $\left\{T^{*}, \dot{m}\right\}_{i}$ on Pareto front corresponds to a certain realization of the tank water temperature $T(t)$, tap water temperature $T_{\mathrm{d}}(t)$, thermal discomfort $D_{T}(t)$, electricity consumption $E_{e}(t)$ and heat losses $Q_{\text {loss }}(t)$. 


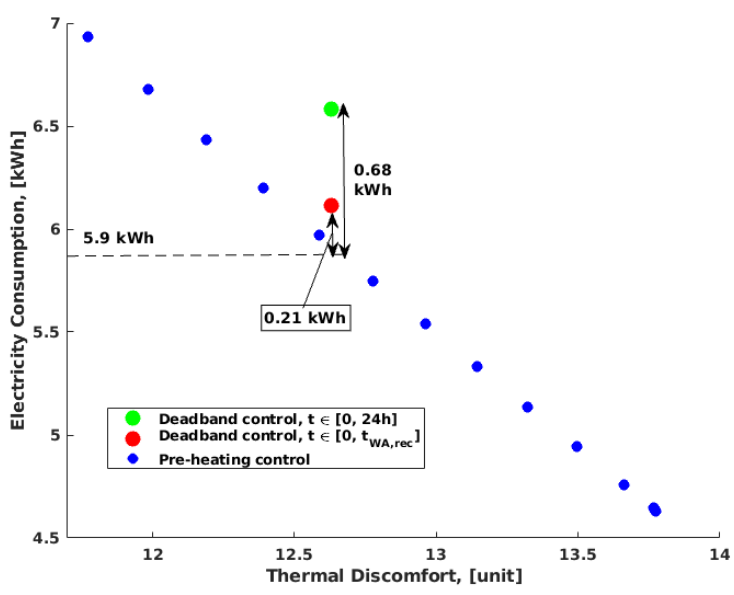

(a)

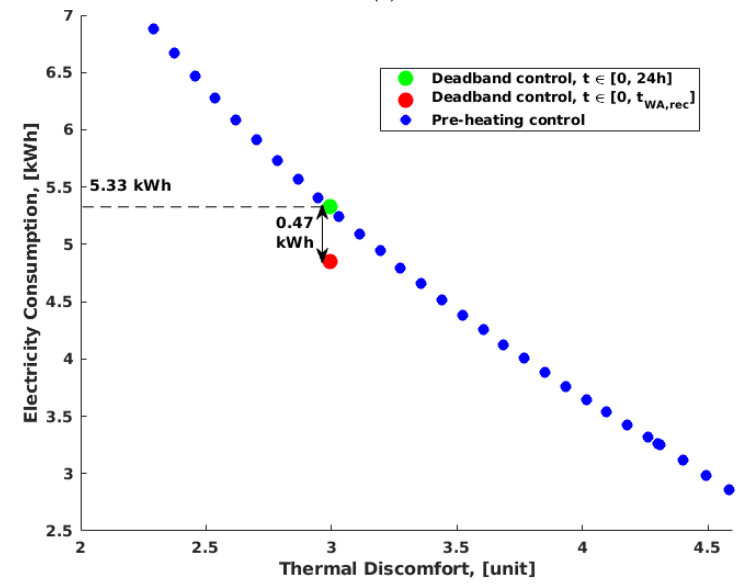

(b)

Figure 4.7: Pareto fronts (a) $\dot{m}_{\mathrm{d}}=25[\mathrm{~L} / \mathrm{min}], \Delta t_{\mathrm{WA}}=25[\mathrm{~min}]$, (b) $\dot{m}_{\mathrm{d}}=25[\mathrm{~L} / \mathrm{min}]$, $\Delta t_{\mathrm{WA}}=10[\mathrm{~min}]$.

The graphs of tank water temperature $T(t)$ and electric energy consumption $E_{e}(t)$ functions for two solutions under approximately equal comfort levels $D_{T \text {,pre }} \approx D_{T \text {,reg. oper. }}$ in Figure 4.7(a) and Figure 4.7(b) are plotted in Figure 4.9 and Figure 4.10.

To evaluate the quality of the obtained solutions, we calculated the values of the term $Q_{\text {loss }}$ in Equation 4.4 in the domain of the function $D_{T}(T, \dot{m})$ while applying the ranges $T \in\left[T_{\mathrm{cw}}, T_{\text {wh,max }}\right]$ and $\dot{m} \in\left[0, \dot{m}_{\mathrm{d}}\right]$. Figure 4.11 shows the surface of the function $D_{T}(T, \dot{m})$ in the considered domain for the WA in Figure 4.12. The horizontal plane corresponds to the user thermal discomfort level under $\gamma=0.58$. The possible values of the function $Q_{\text {loss }}$ in the considered domain and represented as a heat map along with the contours of the function $D_{T}(T, \dot{m})$ are shown together in Figure 4.12. The gray region in Figure 4.12 is a "no-go" zone of pairs $\{T, \dot{m}\}$ that render $T_{\mathrm{d}}(0)>T_{\text {cmax }}$ and that are excluded by constraint in Equation 5.7. The resulting trajectory of solutions is shown in Figure 4.12 (in green). 


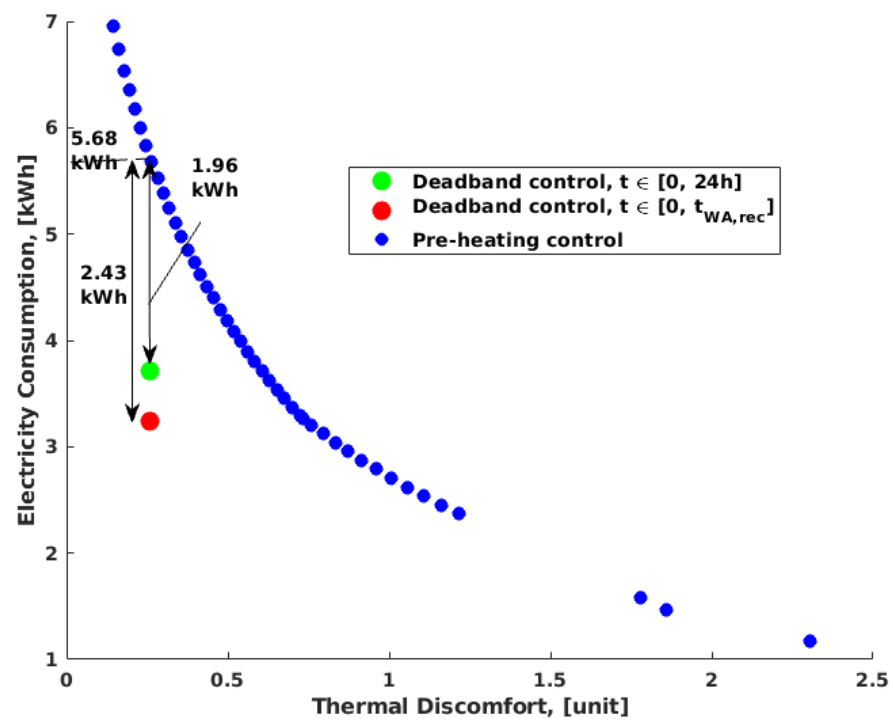

Figure 4.8: Pareto front $\dot{m}_{\mathrm{d}}=10[\mathrm{~L} / \mathrm{min}], \Delta t_{\mathrm{WA}}=10[\mathrm{~min}]$.

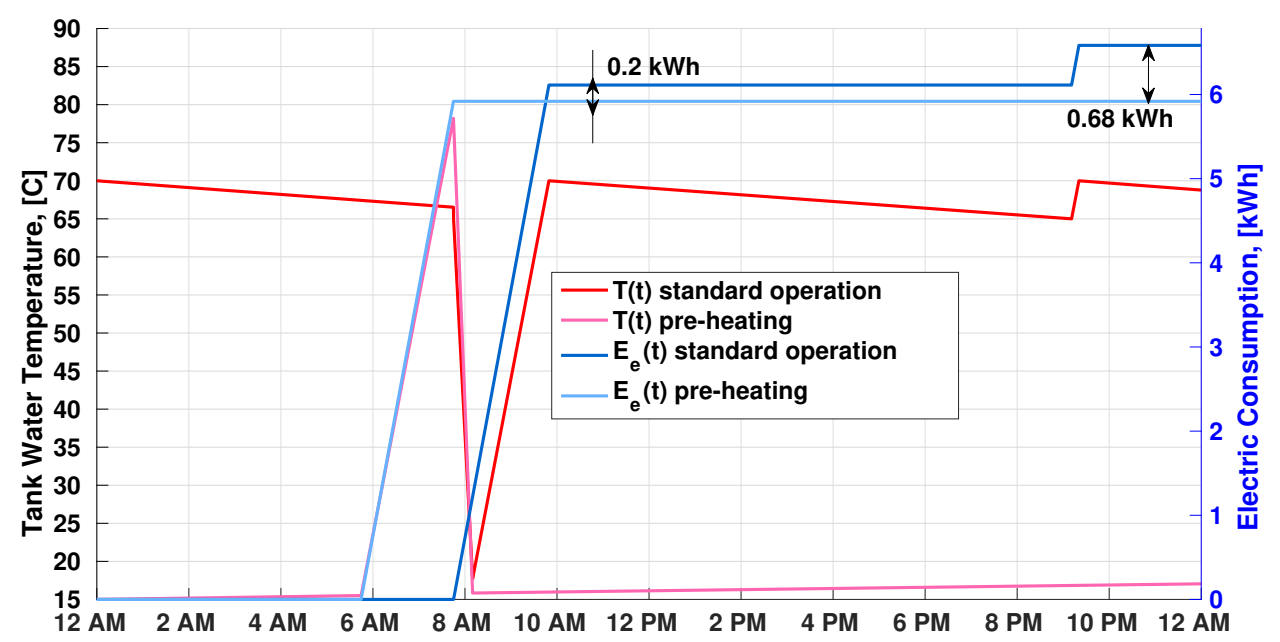

Figure 4.9: Tank water temperature $T(t)$ and electricity consumption $E_{e}(t)$ for WA $\dot{m}_{\mathrm{d}}=25$ $[\mathrm{L} / \mathrm{min}], \Delta t_{\mathrm{WA}}=25[\mathrm{~min}]$.

Each of the solutions on this trajectory refers to a different levels of the user thermal discomfort $D_{T}$, heat losses $Q_{\text {loss }}$ and electricity consumption $E_{e}$ on the Pareto front. 


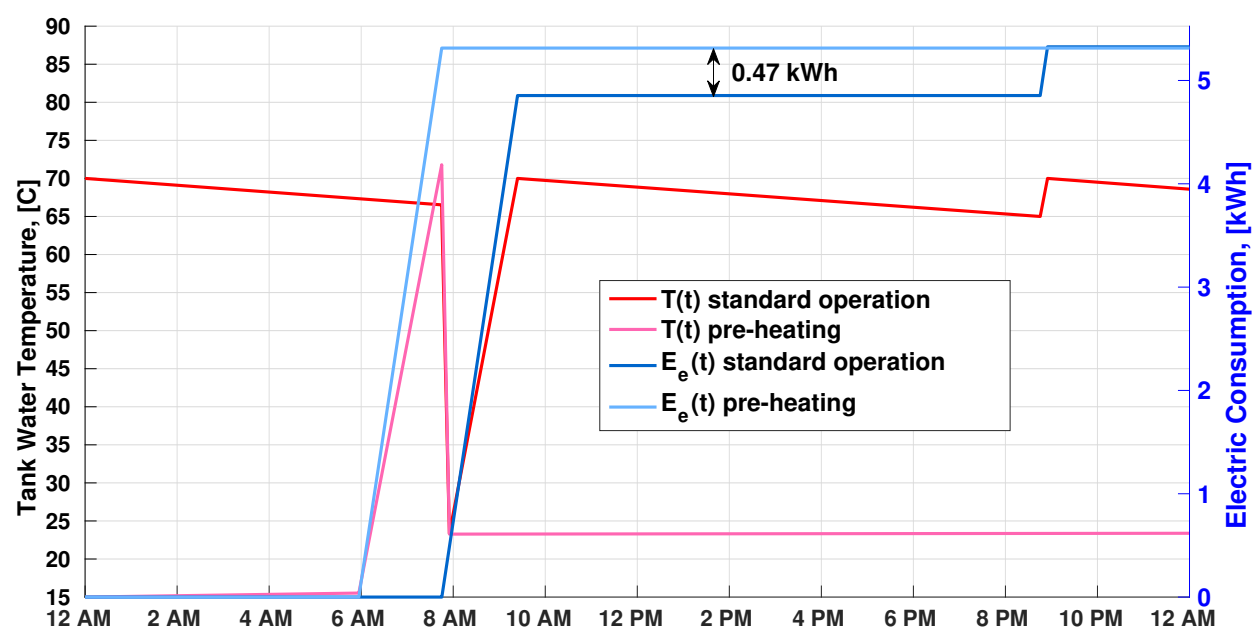

Figure 4.10: Tank water temperature $T(t)$ and electricity consumption $E_{e}(t) \dot{m}_{\mathrm{d}}=25$ $[\mathrm{L} / \mathrm{min}], \Delta t_{\mathrm{WA}}=10[\mathrm{~min}]$.

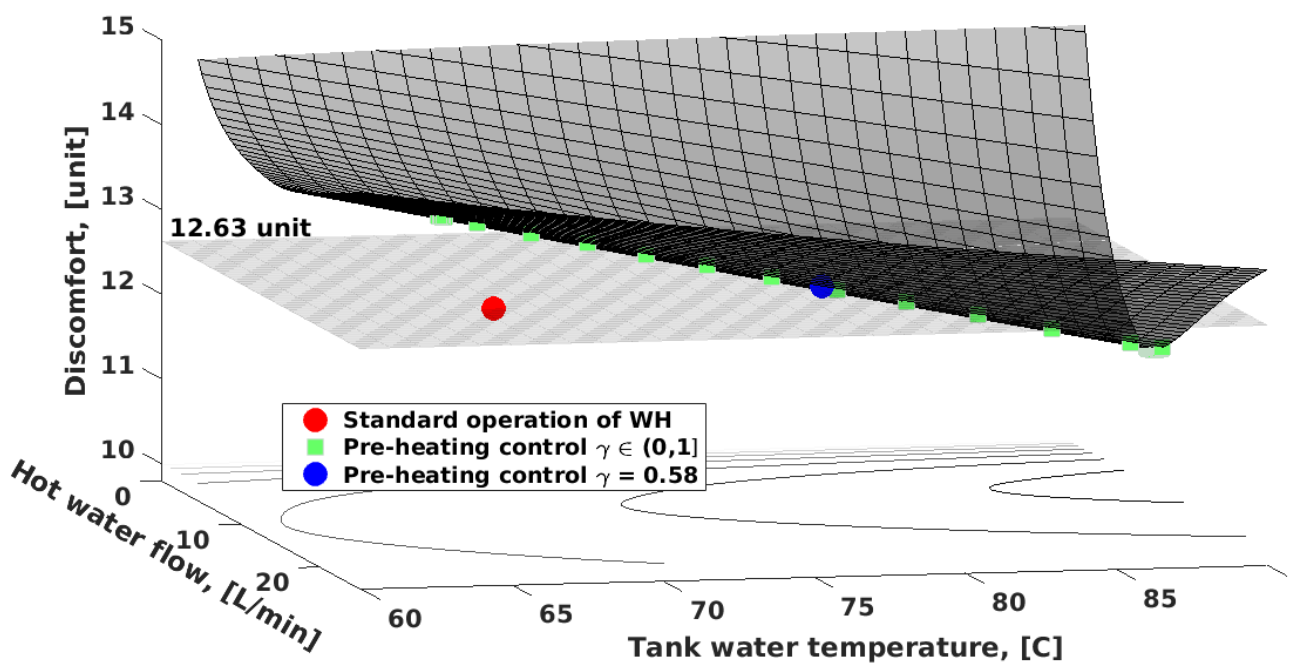

Figure 4.11: Thermal discomfort function $D_{T}(T, \dot{m})$ and heat losses $Q_{\text {loss }}$ for WA $\dot{m}_{\mathrm{d}}=25$ $[\mathrm{L} / \mathrm{min}], \Delta t_{\mathrm{WA}}=25[\mathrm{~min}]$. 


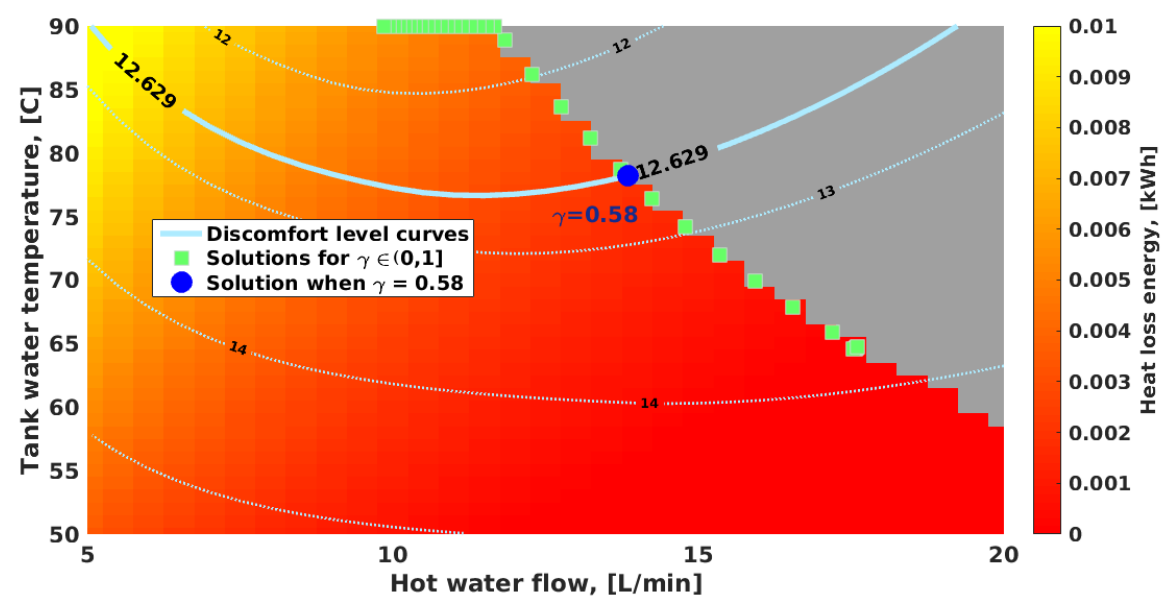

Figure 4.12: Thermal discomfort function domain $D_{T}(T, \dot{m})$ and heat losses $Q_{\text {loss }}$ for WA $\dot{m}_{\mathrm{d}}=25[\mathrm{~L} / \mathrm{min}], \Delta t_{\mathrm{WA}}=25[\mathrm{~min}]$.

\section{Extended Simulations}

Further we conducted a series of extended simulations for WAs with different tap water flow rates $\dot{m}_{\mathrm{d}}$ and durations $\Delta t_{\mathrm{WA}}$ in order to evaluate the impact of the pre-heating control on the user thermal comfort, electricity consumption and heat losses.

In our simulations, we focused only on those pre-heating solutions $\left\{T^{*}, \dot{m}^{*}\right\}$ that were equally or more beneficial than the solutions of the deadband control, i.e. those solutions that provided the same or improved levels of $D_{T}, E_{e}$ and $Q_{\text {loss }}$ as compared to the deadband control. These solutions lay on Pareto front lower and to the left side of the resultant levels of $D_{T}$ and $E_{e}$ of the deadband control in Figure 4.9. To limit the search space and make it possible to compare two types of control, we introduced an additional constraint by requesting that the level of thermal comfort associated with any pre-heating solution should not be worse than thermal comfort rendered by the deadband control. This extra constraint can be expressed as follows:

$$
D_{T} \leq D_{T, \text { reg. oper. }}
$$

where $D_{T}$ stands for the user thermal discomfort in the pre-heating control; $D_{T \text {,reg. oper. }}$ denotes the user thermal discomfort in case of the deadband control.

The right side of inequality in Equation 5.10 implies that the level of the thermal

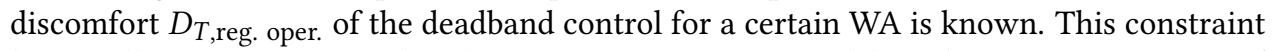
hence allows to compare the electricity consumption and heat losses in two types of control. Figure 4.9 demonstrates the electricity savings and the heat losses delivered by the pre-heating control in comparison with the deadband control under distinct levels of the thermal comfort. After introducing the constraint in Equation 5.10 for the pre-heating control, electricity savings and the heat losses changed as shown in Figure 4.13(b). 


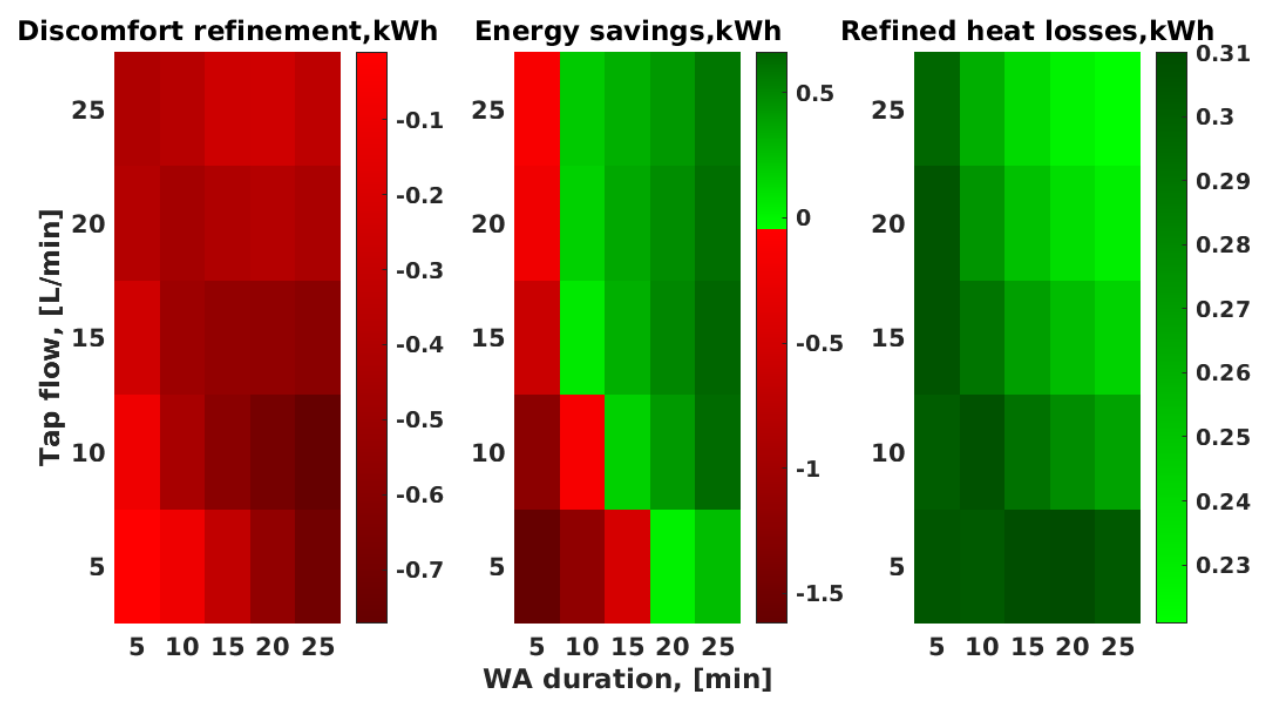

(a)
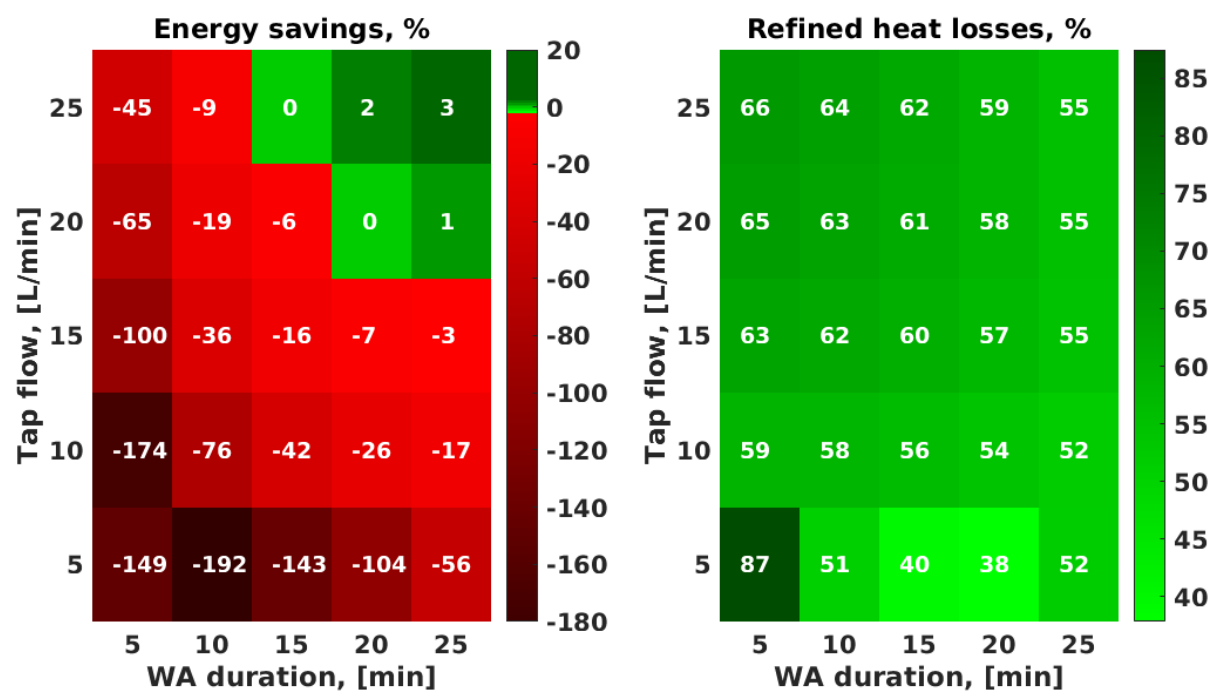

(b)

Figure 4.13: Changes of $D_{T}, E_{e}$ and $Q_{\text {loss }}$ under $U A=0.95$ and $\gamma=0.58$ at $t=t_{\mathrm{WA} \text {,rec. }}$ as compared to deadband control (a) all solutions, (b) solutions with $D_{T} \approx D_{T \text {,reg. oper. }}$.

We then carried the same simulations as above but for the planning horizon extended to one day. Electricity savings and delta of the heat losses achieved by the pre-heating control at $t=24[\mathrm{~h}]$ in comparison with the deadband control are shown in Figure 4.14. 

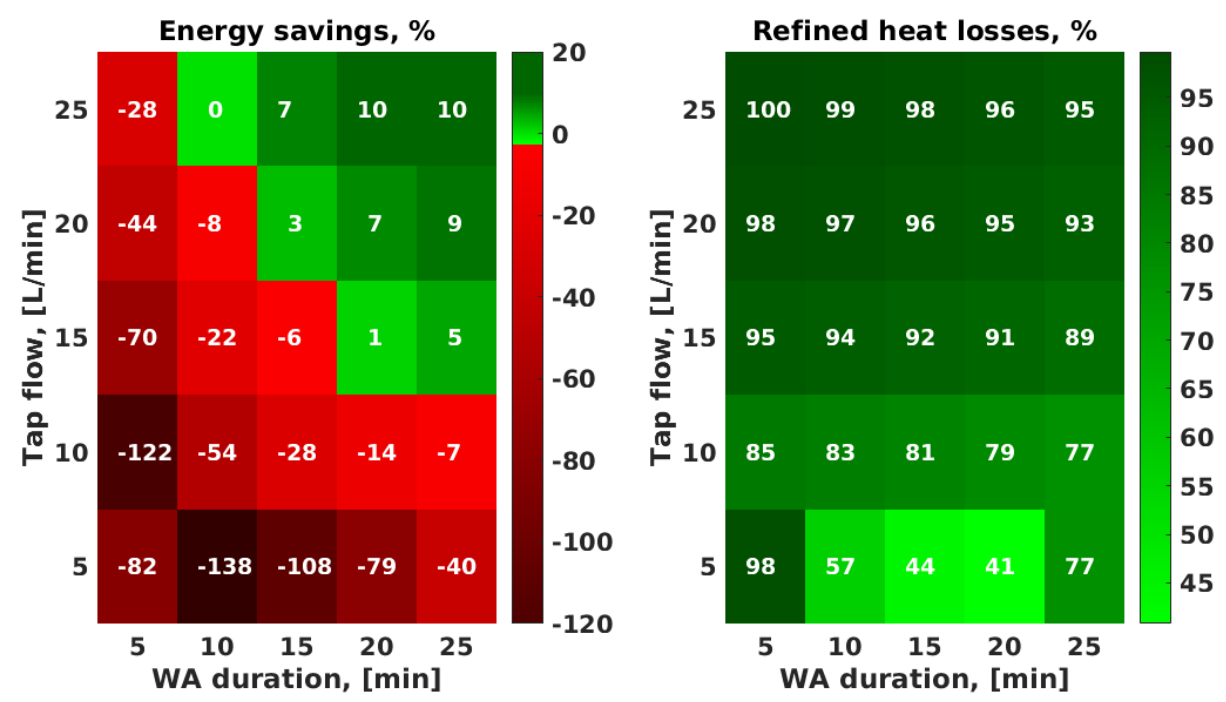

Figure 4.14: Daily changes of $E_{e}$ and $Q_{\text {loss }}$ as compared to deadband control under

$$
U A=0.95 \text { and } \gamma=0.58 \text {. }
$$

Another factor that influences the electricity consumption and the heat losses is the insulation of the $\mathrm{WH}$. We therefore applied two values of the heat loss coefficient $U A$ $(U A=0.085$ and $U A=1.267)$ in the thermodynamic model of the $\mathrm{WH}$ (Equation 2.1). Electricity savings and changes in the heat losses yielded by the pre-heating control in case of different $U A$ values as against the deadband control (under the same $U A$ values) are illustrated in Figure 4.15(a) and Figure 4.15(b).

\subsubsection{Results Discussion and Conclusion}

As it can be seen from Pareto plots in Figure 4.7, the pre-heating control cannot provide zero user thermal discomfort in all scenarios of hot water usage. It can be explained by a finite volume of the water tank $(80 \mathrm{~L})$ and the safety temperature constraint in Equation 5.5 that restrict the amount of the thermal energy in the WH tank.

The pre-heating strategy gives certain electricity savings under the same level of comfort as compared to the deadband control in case of intense WA in Figure 4.7(a). However, electricity consumption of the pre-heating becomes equal to daily electricity consumption of the deadband control in Figure 4.7(b) and exceeds it for the less intense WA in Figure 4.8. One can conclude that application of the pre-heating control can be advantageous in case of intense WAs, i.e. for long WAs ( $\left.t_{\mathrm{WA}} \geq 20[\mathrm{~min}]\right)$ and/or WAs with high tap flow rate $\left(\dot{m}_{\mathrm{d}} \geq 20[\mathrm{~L} / \mathrm{min}]\right)$.

Looking at the solution for intense WA in Figure 4.9, we can conclude that the preheating overplays the deadband control in terms of the electricity consumption by 0.2 [kWh] already after recovery of the tank water temperature $t=t_{\mathrm{WA}, \text { rec. }}$ and by 0.68 [kWh] in the end of the day. On the other hand, Figure 4.10 shows that even though the pre-heating may be an expensive solution in case of a short planning horizon $t=t_{\mathrm{WA} \text {,rec., }}$, accumulated heat losses may make the pre-heating control equally (or even more) efficient than the 

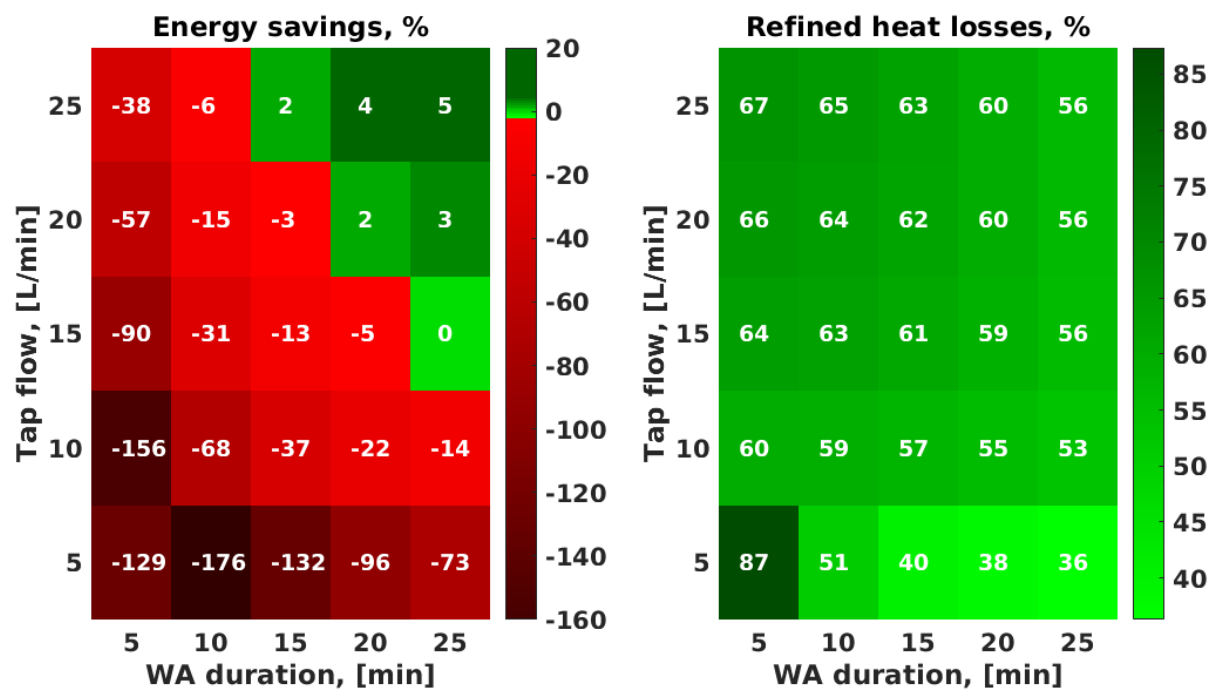

(a)
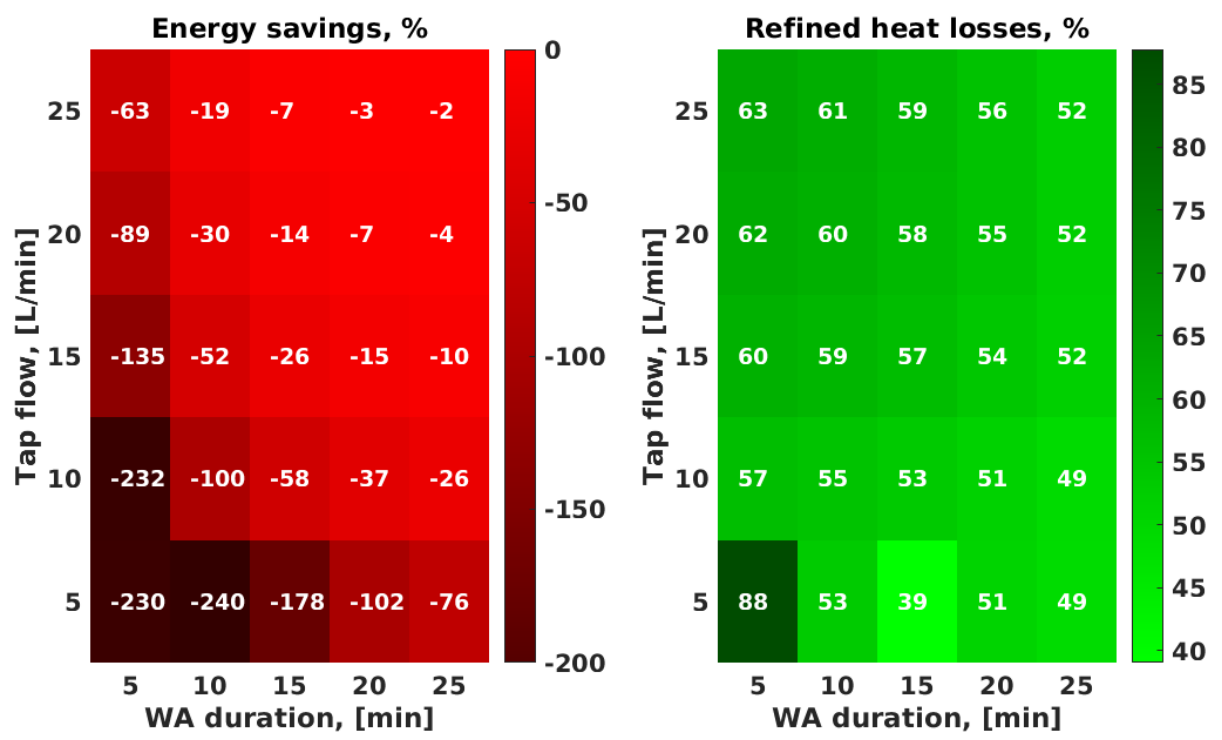

(b)

Figure 4.15: Changes of $E_{e}$ and $Q_{\text {loss }}$ at $t=t_{\mathrm{WA}, \text { rec. }}$ as compared to deadband control under different insulation coefficients (a) $\mathrm{UA}=1.267$, (b) $\mathrm{UA}=0.085$.

deadband control in a long-term perspective.

The surface and the contours of the thermal discomfort function $D_{T}(T, \dot{m})$ in Figure 4.11 point to: (a) multiple solutions $\left\{T^{*}\left(t_{\mathrm{WA}}\right), \dot{m}^{*}\right\}$ that can provide the same level of the thermal comfort, (b) the existence of a global minimum of the function $D_{T}(T, \dot{m})$ that can be achieved 
by multiple solutions, (c) multiple pre-heating solutions that provide better thermal comfort level than the deadband control, while consuming less electricity. These solutions lay below the horizontal plane in Figure 4.11 and correspond to those solutions on Pareto front which are below and to the left side from the the discomfort $D_{T, \text { reg. oper. }}$ and consumption $E_{e, \text { reg. oper. }}$ of the deadband control indicated by the red marker in Figure 4.7(a).

At the same time, the minimum of the heat loss function $Q_{\text {loss }}(T, \dot{m})$ in Figure 4.12 (red color) and the location of the minimum of the function $D_{T}(T, \dot{m})$ do not coincide. Which means that that the goals of minimizing the user thermal discomfort and minimizing the heat losses (and conjuncted minimization of the electricity consumption) are indeed conflicting. It can be also concluded that the found solutions comply with the problem constraints in Equation 5.5 - Equation 5.7.

The comparison of the electricity savings and heat losses rendered by the pre-heating control under (approximately) the same levels of the user thermal comfort as in the deadband control indicates that water pre-heating is more advantageous than the deadband control in terms of the electricity consumption for intense WAs $\left(\dot{m}_{\mathrm{d}}>20[\mathrm{~L} / \mathrm{min}]\right.$ and $\Delta t_{\mathrm{WA}} \geq 20$ [min]) as can be see in Figure 4.13(b). Furthermore, the pre-heating control deliveres lower heat losses for short WAs with low tap water flow rates than for intense WAs mainly because the water should be heated to higher temperatures to satisfy the user thermal comfort for intense WAs. The pre-heating control in all the cases gave lower heat losses than the deadband control.

Considering that the heat losses continuously impact energy consumption and that average temperature at the end of the WA in case of the water pre-heating is much lower than the WH setpoint temperatures, the pre-heating control is capable to yield higher energy efficiency on a daily planning horizon. Comparing Figure 4.14 with Figure 4.13(b), shows how previously inefficient WA categories are 'becoming' more efficient in a longterm perspective due to the increase of the accumulated heat losses during the standard operation. Specifically, the pre-heating control brings electricity savings already for WAs with the tap water flow rates $\dot{m}_{\mathrm{d}}>15[\mathrm{~L} / \mathrm{min}]$ and duration $\Delta t_{\mathrm{WA}}>15[\mathrm{~min}]$.

The second round of simulations was aimed at investigating the effect of the $\mathrm{WH}$ insulation on the efficiency of pre-heating action. The results represented in Figure 4.15(a) and Figure 4.15(b) for the case of a medium $(U A=0.95)$ and poor $(U A=1.267)$ insulation show a slight improvement in efficiency of the pre-heating control in terms of $E_{e}$ and $Q_{\text {loss }}$ for the WH with weak insulation, which can be explained by the increased rate of heat losses.

To summarize, the proposed pre-heating mechanism is capable to attain trade-offs between the user thermal comfort and electricity consumption. In the worst-case scenario where the WH tank is initially filled with cold water $\left(T(0)=T_{\mathrm{cW}}\right)$, the pre-heating control outperforms the deadband control of the WH energy-wise for intense WAs. Furthermore, the relative energy efficiency of the pre-heating improves as compared to the deadband control (a) for WHs with poor insulation and (b) with an extension of the planning horizon due to higher operational temperatures of the deadband control.

\subsection{Flow Control Strategy}

In the previous section, we concentrated on a scenario where the user required the tap water in a comfortable range of temperatures. This section focuses on a scenario where a 
nearly stable tap water temperature is desirable by the user.

It is possible to achieve a close to stable tap water temperature $T_{\mathrm{d}} \approx$ const during a hot water activity (WA) by regulating the flow rates of hot and cold water in the mixer tap, for example as it is done in thermostatic mixing valves (TMVs) $[110,111]$ or pressure balance valves (PBVs) [112]. One of the downsides of the standard solutions is that their control is decoupled from the water heating mechanism of the WH which hinders an efficient planning of the hot water production. To make the hot water service more energy and comfort efficient, the control solutions of the water heating mechanism (on the WH side) should be found with respect to solutions of the flow control at the tapping point.

This section introduces a control mechanism for scenarios of the hot water usage where a nearly stable tap water temperature is required. More specifically, the proposed control consolidates a flow control strategy similar to TMVs combined with the water pre-heating control presented in Section 4.4. Two distinct types of control are tied together by means of the tank water temperature variable. If the flow control algorithm finds the best tank water temperature value to be established at the beginning of the WA and suitable for the user-desired tap water temperature value during the WA, the pre-heating control algorithm seeks the best time to start the water heating to the found temperature value. Such a communication scheme allows for finding the solutions of the water heating control with respect to the solutions of the flow control, which is (as it will be shown later) more efficient from the economical and user comfort viewpoints.

Another distinguishing feature of the combined control is that the flow control component can vary the tap water flow rate. In the considered HWS where the tank water temperature inevitably drops during WAs, the user might soon experience the thermal discomfort. To support the user thermal comfort, one of the possibilities is to gradually reduce the tap water flow rate with the progress of the tank water temperature drop. In this case, the user does not suddenly experience a thermal discomfort but rather experiences a gradual drop of the tap water flow which can signalize him about the lack of hot water in the WH tank. However, the existing solutions like TMVs control only the water inflows while treating the outflow of the mixed water as an uncontrollable parameter provided by the user. In contrast to TMVs/PBVs, we remove that limitation in our approach and treat the tap water flow as a control variable along with the hot and cold water flows. The resulting tap water flow rate might, however, become too high or too low for the end-user which should be taken into account. We employ the flow comfort model $D_{\dot{m}_{d}}$ (Equation 3.9) in order to control the water flows with respect to the user-desired flow comfort level.

\subsubsection{Standard Solutions to Support Stable Tap Water Tem- PERATURE}

There is a wide spectrum of engineering solutions to provide a stable tap water temperature $[91,93,110,112]$. The stable water temperature is achieved in TMVs $[110,111]$ by means of a thermosensitive element that senses the temperature of the mixed water in the outlet port (tap water temperature) and reacts to its fluctuations by adjusting the incoming cold and hot water flows. Pressure balance valves (PBVs) are used for the tap water temperature stabilization in response to pressure changes of the hot and cold water flows [112]. Other solutions include electronic taps [113, 114] and instant boiling taps [115] that can be programmed to provide an precise stable temperature of water. One of the 
ways to provide a stable tap water is to regulate the hot and cold water flows at the tapping point, as it is done in TMVs and electronic taps, i.e. to perform a flow control.

One of the most popular solutions, TMVs, rely on the physical properties of the thermosensitive material to provide automatic flow control. When installed in the HWS with a $\mathrm{WH}$, TMVs utilize the thermal energy stored in the WH during a WA. The water heating mechanism realized on the WH side is decoupled from the flow rate control at the tapping point, since two types of control are working independently from each other. Therefore, the water is heated in the WH without regard for the TMV operation. This can lead to overheated or underheated tank water, which is inefficient from the economical and user comfort points of view. If the tank water temperature at the beginning of a WA is too high, the flow regulation will not utilize all the heat efficiently and its residuals will contribute to the heat losses. In case the tank water is too cold, the TMV will not be capable to maintain the user-desired tap water temperature. As such, standalone implementation of water heating control and flow control lacks efficiency.

Solutions like TMVs are usually installed in a piping system of a dwelling and can serve several tapping points. Which means that (a) hot water at the same temperature is delivered to all taps including areas where higher temperatures may be desirable such as kitchens and laundries, (b) if the TMV temperature setting has to be adjusted, it requires physical access to the pipe where TVM is installed.

Solutions like TMVs/PBVs do not control the outflow water rate. Instead, the end-user sets it manually at the mixer tap according to his needs. In some scenarios it may be, however, advantageous to reduce the tap water flow rate, for example, in order to preserve the user thermal comfort (refer to Section 3.3).

\subsubsection{Flow Control Principle}

We briefly describe a flow control principle (also inherent to TMVs) to attain a nearly stable tap water temperatures in a considered HWS presented in Figure 2.1. By plugging Equation 2.6 in Equation 2.1, the ratio between the incoming hot and cold water flow rates in the mixer tap at any moment $t$ can be expressed as follows:

$$
k(t)=\frac{\dot{m}(t)}{\dot{m}_{\mathrm{cw} 2}(t)}=\frac{T_{\mathrm{d}}(t)-T_{\mathrm{cw}}}{T(t)-T_{\mathrm{d}}(t)} .
$$

Equation 4.7 can be interpreted as follows. To achieve the tap water temperature value $T_{\mathrm{d}}(t) \in\left[T_{\mathrm{cw}}, T(t)\right]$ at time $t$, the ratio $k(t)$ between the hot and cold water flows should be maintained. Importantly, the tank water temperature value $T(t+1)$ at the moment $t+1$ depends on the values $T(t)$ and $\dot{m}(t)$ as it follows from Equation 2.2, which means that the ratio $k(t+1)$ that can provide a nearly stable tap water temperature $T_{\mathrm{d}}(t+1) \approx T_{\mathrm{d}}(t)$ at time $t+1$ depends on the values $T(t)$ and $\dot{m}(t)$ too. The flow control principle for $k(t)$ is hence based on the recursion.

Further analysis of Equation 4.7 allows to derive various possibilities of managing the demanded temperature $T_{\mathrm{d}}(t)$ by manipulating the hot water flow rate $\dot{m}(t)$ and cold water flow rate $\dot{m}_{\mathrm{cw} 2}$ in the mixer tap as presented in Table 4.1.

Table 4.1 highlights the possibility (Case \#3) to provide the user with a stable tap water temperature by gradually increasing the hot water flow $\dot{m}(t)$ and lowering the cold water inflow $\dot{m}_{\text {cw2 }}$ during the WA. 
Table 4.1: Possibilities of water flow control (symbol $\downarrow / \uparrow$ denotes decrease/increase of parameter, number of symbols indicates the intensity).

\begin{tabular}{|c|c|c|c|c|}
\hline Case \# & $\dot{m}(t)$ & $\dot{m}_{\mathrm{cw} 2}(t)$ & $T_{\mathrm{d}}(t)$ & $T(t)$ \\
\hline 0 & $\downarrow$ & $\uparrow$ & $\downarrow \downarrow \downarrow$ & $\downarrow$ \\
\hline 1 & const & const & $\downarrow \downarrow$ & $\downarrow \downarrow$ \\
\hline 2 & $\uparrow$ & $\downarrow$ & $\downarrow$ & $\downarrow \downarrow \downarrow$ \\
\hline 3 & $\uparrow \uparrow$ & $\downarrow \downarrow$ & const & $\downarrow \downarrow \downarrow \downarrow$ \\
\hline 4 & $\uparrow \uparrow \uparrow$ & $\downarrow \downarrow \downarrow$ & $\uparrow$ & $\downarrow \downarrow \downarrow \downarrow \downarrow$ \\
\hline
\end{tabular}

The simulations for various control options in Table 4.1 are illustrated in Figure 4.16.

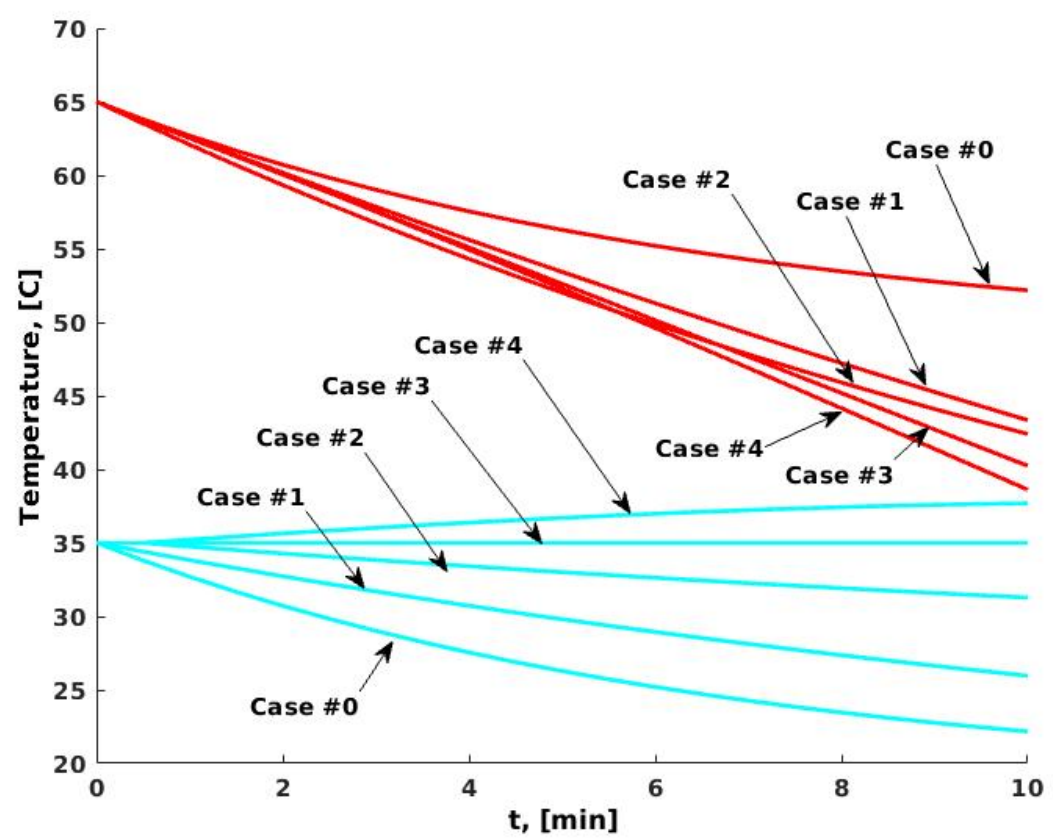

Figure 4.16: Different options to provide flow control (tank water temperature in red, tap water temperature in cyan).

\subsubsection{Problem of Coupling Flow Control with Water Heating}

Given all time- and comfort-related parameters of the expected WA (e.g., from an ideal forecast), the flow control solely can be implemented by a flow controller of the mixer tap that regulates the ratio $k(t)$ in a step-wise manner. Figure 4.17 shows how the flow control can be implemented in case of the desired tap water temperature $T_{\mathrm{d}}(t) \approx T_{\mathrm{d} \text {,ref }}=45^{\circ} \mathrm{C}$.

We argue that in scenarios where a limited amount of hot water is available and there is a need for a stable water temperature (e.g., showering) it may be beneficial to reduce the tap flow rate $\dot{m}_{\mathrm{d}}$ thereby contributing to the user thermal comfort. However, by making the tap water flow rate variable, we create a risk that a modified tap water flow may become 
uncomfortable for the user, which should be accounted for in control. For example, possible solutions of the flow control for the 7-minute WA and the user-desired water temperature $T_{\mathrm{d} \text {,ref }}=45^{\circ} \mathrm{C}$ are illustrated in Figure 4.18(a). As it can be seem from Figure 4.18(a), there can exist multiple solutions the can provide the tap water at the required $45^{\circ} \mathrm{C}$ and each of which resulting in a different tap water flow. Both of the shown control solutions lead to the tap water flows below the desired $10[\mathrm{~L} / \mathrm{min}]$, causing the flow discomfort for the user (Figure 4.18(b)). Therefore, besides providing the user-desired stable tap water temperature, the flow control should satisfy the user flow comfort.

As it has been discussed earlier in Section 4.5.1, it essential for the efficient hot water provision that the water heating works in tandem with the flow control. In other words, the water heating control should establish such a tank water temperature value at the beginning of the WA that (a) allows to provide the user-desired tap water temperature, and (b) allows to minimize the heat wasted for the heat losses.

All things considered, the problem of the combined water heating and flow control can be formulated as follows.

\section{COMBINED CONTROL PROBLEM}

Find the best tank water temperature and water flow rates in the mixer tap to be respectively established at the WA startup and during the WA so that they provide the required nearly stable tap water temperature while minimizing the flow comfort disruptions and heat losses during the WA.
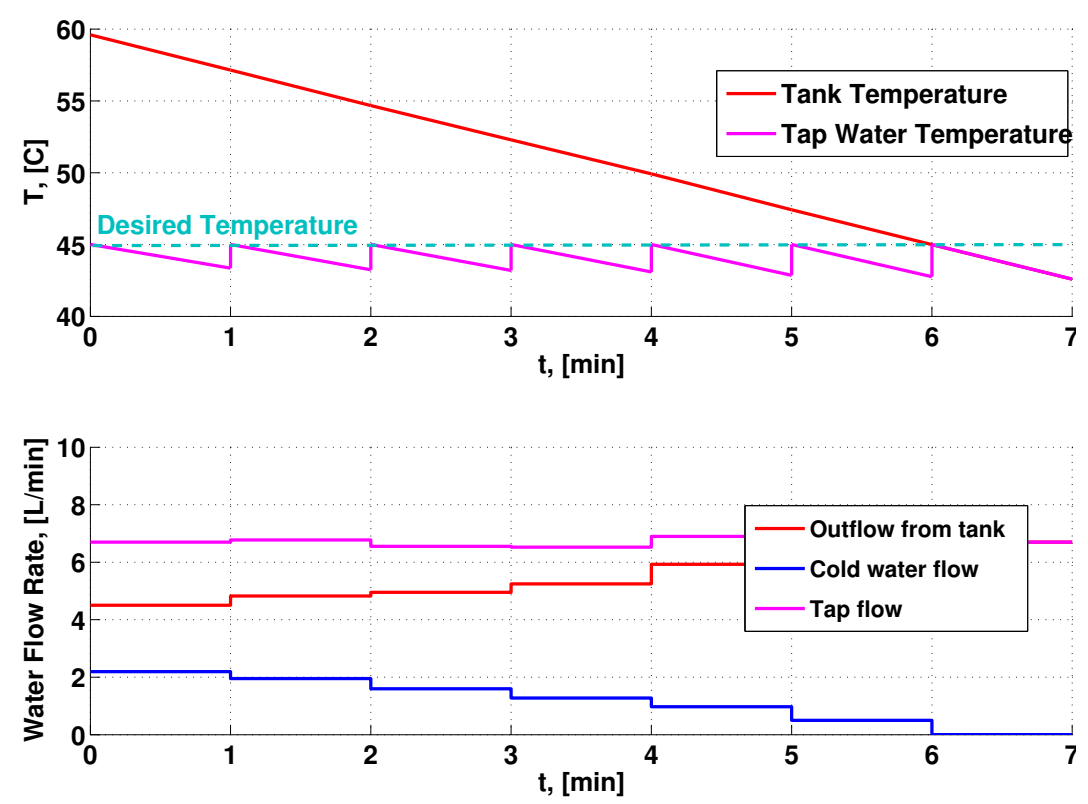

Figure 4.17: Flow control in the mixer tap. 


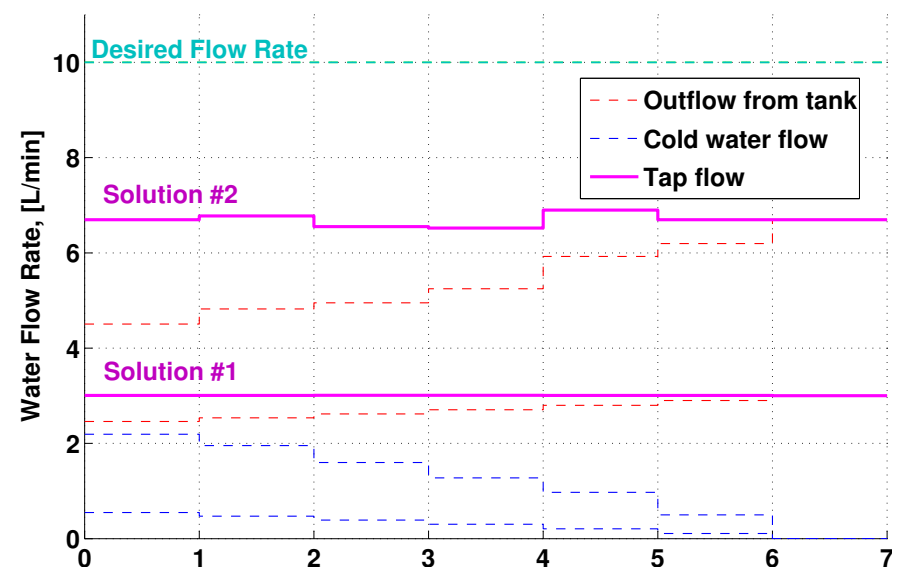

(a)

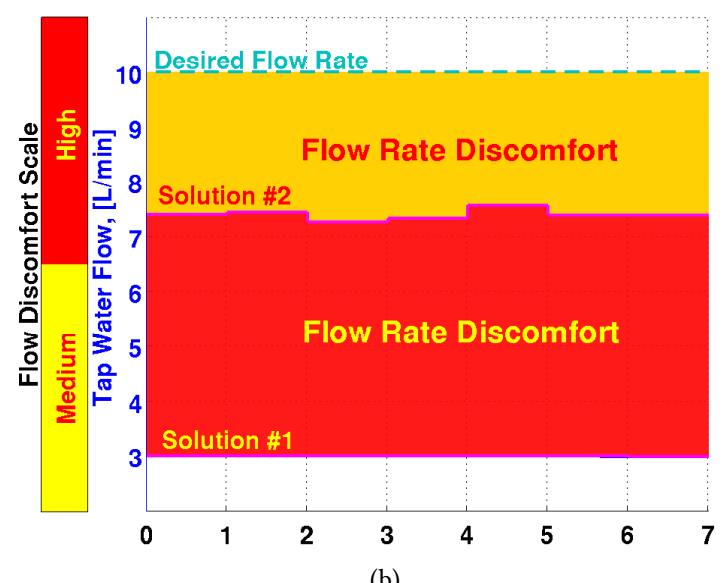

(b)

Figure 4.18: Multiple solutions and flow rate discomfort zones.

\subsection{Proposed Combined Pre-heating and Flow Con- TROL}

Unlike the analog (continuous) flow control of TMVs which relies on a response of the thermosensitive element to water temperature variations, the proposed flow control explicitly finds the best values of the water flows and the tank water temperature in a step-wise manner.

Consider a thermodynamic process during which the temperature inside the tank $T(t)$ gradually drops due to the open hot water tap. We divide the timeline of a WA with duration $\Delta t_{\mathrm{WA}}$ [min] evenly into $N$ intervals of size $\Delta t=\frac{\Delta t_{\mathrm{WA}}}{N}$. Let $i \in[1, N]$ be the index of any interval so that $t_{1}=t_{\mathrm{WA}}$ and $t_{N}=t_{1}+\Delta t_{\mathrm{WA}}-\Delta t$. The function $T(t)$ is then represented by a sequence of values $\left\{T_{1}, T_{2}, \ldots, T_{i}, \ldots, T_{N}, T_{N+1}\right\}$ on a discretized WA timescale. As it follows from Equation 2.2, each value $T_{i}$ is determined by the water flow $\dot{m}$ and the temperature $T_{i-1}$ from the previous interval $i-1$. 
In general, we need to find (i) the best tank water temperature $T\left(t_{1}\right)$ at the beginning of the WA $t_{\mathrm{WA}}$ that determines the amount of heat to be pre-stored, and (ii) the way to utilize that heat by regulating the hot water and cold water flows $\left(\dot{m}(t), \dot{m}_{\mathrm{cw}}(t)\right)^{4}$ during the WA so that the following objectives are attained:

1. the tap water temperature at the beginning of each $i$-th step is equal to the userdesired temperature $T_{\mathrm{d} \text {,ref; }}$;

2. the total flow discomfort $D_{\dot{m}_{d}}$ that the user may experience during the WA is minimized;

3. the total heat losses $Q_{\text {loss }}$ during the pre-heating phase and in the progress of the WA are minimized.

More specifically, we need to find a solution at every $i$-th step represented by a triplet $X_{i}^{*}=\left\{T\left(t_{i}\right), \dot{m}\left(t_{i}\right), \dot{m}_{\mathrm{cw}}\left(t_{i}\right)\right\}$ that includes the tank water temperature $T\left(t_{i}\right)$ to be established at the beginning of the $i$-th step and the hot and cold water flow rates $\left(\dot{m}\left(t_{i}\right), \dot{m}_{\mathrm{cw}}\left(t_{i}\right)\right)$ in the mixer tap to be maintained during that step. It is important to note that the solution $X_{i}^{*}$ at any step $i$ cannot be found standalone without considering the solutions on previous steps $1,2, \ldots, i-1$ because all the temperature variables $T\left(t_{1}\right), T\left(t_{2}\right), \ldots, T\left(t_{N}\right)$ are connected together by a single thermodynamic process. In other words, the solution found on the current step will influence the solutions on all the next steps during the WA. Therefore, we need to find such a series of interconnected solutions $\left\{X_{1}^{*}, X_{2}^{*}, \ldots, X_{N}^{*}\right\}$ on $N$ steps of the WA timescale that fulfills the above objectives (1)-(3).

We can approach this problem by applying the dynamic programming method. The problem can be then broken down into a sequence of $N$ nested sub-problems to be solved at each time step $i \in[1, N]$ so that each solution $X_{i}^{*}, i \in[1, N]$ should provide:

- the user-desired tap water temperature $T_{\mathrm{d}}\left(t_{i}\right)$ at the beginning of $i$-th step;

- the minimum flow discomfort $D_{\dot{m}_{d}}\left(t_{i}, t_{i+1}\right)$ for the user during the $i$-step;

- the minimum heat losses $Q_{\text {loss }}\left(t_{i}, t_{i+1}\right)$ during the $i$-step.

In terms of the dynamic programming theory, each value $T_{\mathrm{d}}\left(t_{i}\right)$ determines the state of the system at step $i$, the flow rates $\left(\dot{m}\left(t_{i}\right), \dot{m}_{\mathrm{cw}}\left(t_{i}\right)\right)$ are control variables and $T\left(t_{i}\right)$ is an auxiliary variable (unknown) that binds the state and control variables together. Every solution $X_{i}$ can be evaluated by means of some value function $F_{i}=F\left(X_{i}\right)$. In case the value function $F_{N}=F\left(X_{N}\right)$ at the last time step is known, one can find the values at previous times $i=N-1, N-2, \ldots, 2,1$ by working backwards, using a recursive Bellman equation [116].

By noticing that the tank water temperature $T\left(t_{N}\right)$ at the beginning of the last step $t_{N}$ should be at least equal to the desired tap water temperature $T_{\mathrm{d} \text {,ref }}$ (in order to satisfy the requirement (1)), our problem can be solved recursively backwards for each step $N-1, N-2, \ldots, 2,1$ until the initial tank water temperature $T\left(t_{i}\right)$ is found. In our case, the final solution $X_{1}^{*}=\left\{T\left(t_{1}\right), \dot{m}\left(t_{1}\right), \dot{m}_{\mathrm{cw}}\left(t_{1}\right)\right\}$ at the step $i=1$ comprises the wanted tank water temperature value $T\left(t_{1}\right)$ to be established by the end of the water pre-heating procedure. In that regard, the pre-heating control of the $\mathrm{WH}$ is responsible for heating the water up

\footnotetext{
${ }^{4}$ Hereinafter, we refer to the cold water flow rate $\dot{m}_{\mathrm{cw} 2}$ in the mixer tap by using $\dot{m}_{\mathrm{cw}}$ notation for simplicity.
} 
to $T\left(t_{1}\right)$ upfront the WA and the flow control during the WA regulates the water flows $\dot{m}\left(t_{i}\right), \dot{m}_{\mathrm{cw}}\left(t_{i}\right), i \in[1, N]$ with the progress of the WA to provide a nearly stable tap water temperature.

These two types of control can be implmented on physically different and, possibly, remote nodes, namely the WH Node and the Tap Node. If WH Node located at the WH implements the pre-heating control, the Tap Node executes the flow control at the tapping point. The WH Node and the Tap Node can communicate with each other in the following scheme:

1. based on the information about the expected WA, the Tap Node finds the best solutions $\left\{X_{N}^{*}, X_{N-1}^{*}, \ldots, X_{1}^{*}\right\}$ in terms of the objectives (1)-(3) at the beginning of the planning period. The found temperature value $T\left(t_{1}\right)$ is returned to the WH Node.

2. using $T\left(t_{1}\right)$ as input, the WH Node tracks the current tank water temperature, computes the best time to start the water pre-heating and initiates the water heating so that $T\left(t_{1}\right)$ is established right at the WA startup.

\subsubsection{Problem Constraints}

Taking into account the user requirement for the stable tap water temperature during a WA, i.e. $T_{\mathrm{d}}(t) \approx T_{\mathrm{d} \text {,ref }}=$ const , we require that the flow control should maintain the user-desired tap water temperature value at beginning of every $i$-th step:

$$
T_{\mathrm{d}}\left(t_{i}\right)=T_{\mathrm{d}, \mathrm{ref}}, i \in[1, N] \text {. }
$$

After substituting the tap water temperature $T_{\mathrm{d}}\left(t_{i}\right)$ Equation 2.7 in Equation 4.8, we can rewrite the above constraint as:

$$
\frac{\dot{m}\left(t_{i}\right)}{\dot{m}\left(t_{i}\right)+\dot{m}_{\mathrm{cw}}\left(t_{i}\right)}\left(T\left(t_{i}\right)-T_{\mathrm{cw}}\right)+T_{\mathrm{cw}}=T_{\mathrm{d}, \mathrm{ref}}
$$

The water temperature inside the tank at any step $i$ is related to the temperature at $i+1$ via Equation 2.2 of the thermodynamic process:

$$
T\left(t_{i+1}\right)=\left(\frac{\alpha}{\beta}+T\left(t_{i}\right)\right) e^{\beta \Delta t}-\frac{\alpha}{\beta} .
$$

The tank water temperature can take the values from the following range:

$$
T_{\mathrm{cw}} \leq T\left(t_{i}\right) \leq T_{\mathrm{wh}, \max } .
$$

The standard assumption of non-negative water flows in the HWS can be written as:

$$
\begin{gathered}
\dot{m}\left(t_{i}\right) \geq 0, \\
\dot{m}_{\mathrm{cw}}\left(t_{i}\right) \geq 0 . .
\end{gathered}
$$

Furthermore, the outflow from the mixer tap $\dot{m}_{\mathrm{d}}(t)=\dot{m}(t)+\dot{m}_{\mathrm{cw}}(t)$ should be positive during the WA, which can be expressed by the following constraint:

$$
\dot{m}\left(t_{i}\right)+\dot{m}_{\mathrm{cW}}\left(t_{i}\right)>0 .
$$




\subsubsection{ObJective Functions}

The goal is to find the best combinations of variables $X_{i}^{*}=\left\{T\left(t_{i}\right), \dot{m}\left(t_{i}\right), \dot{m}_{\mathrm{cw}}\left(t_{i}\right)\right\}, i \in[1, N]$ that lead to:

1. minimization of the user flow discomfort $D_{\dot{m}_{d}}\left(t_{i}, t_{i+1}\right)$ during each $i$-th step;

2. minimization of the heat losses $Q_{\text {loss }}\left(t_{i}, t_{i+1}\right)$ during each $i$-th step.

while satisfying the constraints formulated in Section 4.6.1.

At this point, we have two objective functions:

1. $\min \left[D_{\dot{m}_{d}}\left(t_{i}, t_{i+1}\right)\right]=\min \left[D_{\dot{m}_{d}}\left(\dot{m}\left(t_{i}\right), \dot{m}_{\mathrm{cW}}\left(t_{i}\right)\right)\right], i \in[1, N]$;

2. $\min \left[Q_{\mathrm{loss}}\left(t_{i}, t_{i+1}\right)\right]=\min \left[Q_{\mathrm{loss}}\left(\dot{m}\left(t_{i}\right), T\left(t_{i}\right)\right)\right], i \in[1, N]$,

that have to be simultaneously satisfied.

We apply a dynamic programming approach by recursively resolving $N$ overlapping subproblems at steps $i \in[1, N]$. More precisely, the solution starts from the last interval with index $i=N$. For step $i=N$, we already know that the tank water tempeature value at the end of the step $T\left(t_{N+1}\right)$ should be equal to $T_{\mathrm{d} \text {,ref }}$ so that $T_{\mathrm{d}}\left(t_{N+1}\right)=T\left(t_{N+1}\right)=T_{\mathrm{d} \text {,ref. }}$. We thus need to decide upon the values of the flow rates $\dot{m}\left(t_{N}\right), \dot{m}_{\mathrm{cw}}\left(t_{N}\right)$ and the tank water temperature $T\left(t_{N}\right)$ that will lead to a given temperature value $T\left(t_{N+1}\right)=T_{\mathrm{d}}$,ref while minimizing objective functions (1) and (2). We do it by plugging the value The unknown decision variables $X_{N}^{*}=\left\{T\left(t_{N}\right), \dot{m}\left(t_{N}\right), \dot{m}_{\mathrm{cW}}\left(t_{N}\right)\right\}$ are found by minimizing two objective

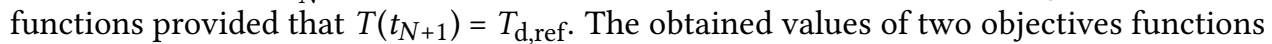
are memorized and fed to the sub-problem at step $N-1$ along with the value of $T\left(t_{N}\right)$. At step $i=N-1$, both the objectives are minimized again and the decision variables $X_{N-1}^{*}=$ $\left\{T\left(t_{N-1}\right), \dot{m}\left(t_{N-1}\right), \dot{m}_{\mathrm{cw}}\left(t_{N-1}\right)\right\}$ are found. The process repeats for $i \in[N-2, N-3, \ldots, 1]$. The resulting value of the decision variable $T\left(t_{1}\right)$ after $N$ iterations is the wanted value of the tank water temperature at the beginning of the WA.

This problem can be formalized as a multi-objective goal-attainment problem [117] to be solved recursively at steps $i \in[N, N-1, \ldots, 1]$. To formulate a goal-attainment problem, we introduce an unrestricted scalar slack variable $\lambda$ and a weight coefficient $\gamma \in[0,1]$ that together control the under- or overattainment of two objective functions. The desired values of the objectives functions are known and ideally should be both equal to zero $D_{\dot{m}_{d}}^{*}=D_{\dot{m}_{d}}\left(X_{i}^{*}\right)=0$ and $Q_{\text {loss }}^{*}=Q_{\text {loss }}\left(X_{i}^{*}\right)=0$. Assuming the values of two objective functions are the output of a single vector function $F_{i}=\left[D_{\dot{m}_{d}}\left(X_{i}^{*}\right), Q_{\text {loss }}\left(X_{i}^{*}\right)\right]$, we can formalize the problem to be solved at each step $i \in[N, N-1, \ldots, 1]$ as follows:

$$
F_{i}=\min \left[\lambda_{i}+F_{i+1}\right], i \in[N, N-1, \ldots, 1]
$$

subject to the constraints in Equation 4.9 - Equation 4.14 and the goal constraints:

$$
\begin{gathered}
D_{\dot{m}_{d}}\left(X_{i}\right)-\gamma \lambda_{i} \leq 0, \\
Q_{\text {loss }}\left(X_{i}\right)-(1-\gamma) \lambda_{i} \leq 0,
\end{gathered}
$$

where $F_{i}$ is a pay-off at the $i$-th step; $D_{\dot{m}_{d}}\left(X_{i}\right)$ stands for the user flow discomfort during the $i$-th step; $\gamma \in(0,1]$ is a weight coefficient that controls a relative under- or overattainment 
Table 4.2: WAs with Varied Duration

\begin{tabular}{|c|c|c|c|}
\hline $\begin{array}{c}\text { Duration, } \\
{[\mathrm{min}]}\end{array}$ & $\begin{array}{c}{\left[\dot{m}_{\mathrm{c} . \min }, \dot{m}_{\mathrm{c} \text {.max }}\right],} \\
{[\mathrm{L} / \mathrm{min}, \mathrm{L} / \mathrm{min}]}\end{array}$ & $\begin{array}{c}{\left[\Delta_{\mathrm{tol}}^{-} \Delta_{\mathrm{tot}}^{+}\right],} \\
{[\mathrm{L} / \mathrm{min},} \\
\mathrm{L} / \mathrm{min}]\end{array}$ & $T_{\mathrm{d}, \mathrm{ref},}{ }^{\circ} \mathrm{C}$ \\
\hline 7 & {$[10,12]$} & {$[-2,0]$} & 45 \\
\hline 15 & & & 45 \\
\hline
\end{tabular}

of the objectives, i.e. controls the importance of satisfaction of either of two objectives; $Q_{\text {loss }}\left(X_{i}\right)$ denotes the heat losses during the step $i$.

As it can be seen from Equation 4.16 and Equation 4.17, the weight coefficient $\gamma$ controls the degree of satisfaction of the objectives of minimizing $D_{\dot{m}_{d}}\left(X_{i}\right)$ and $Q_{\text {loss }}\left(X_{i}\right)$. That is to say, the relative magnitude of $\gamma$ determines how near $D_{\dot{m}_{d}}\left(X_{i}\right)$ will be to the best possible value equal to zero. By setting a small weight $\gamma$, we give the optimization solver more accent on minimization of the user flow discomfort, while relaxing the objective to minimize the heat losses. On the contrary, big values of $\gamma$ force the solver to focus on minimization of the heat losses, while softening the need to minimize the user flow discomfort.

After solving the problem at final step $i=1$, we pass the obtained value $T\left(t_{\mathrm{WA}}\right)=T\left(t_{1}\right)$ to the WH Node that performs the water pre-heating from some initial tank water temperature $T(0)$ to the found $T\left(t_{\mathrm{WA}}\right)$ within the time in Equation 2.3.

\subsubsection{Performance Evaluation}

We aim at evaluating the quality of solutions attained by the proposed control in a scenario where a stable tap water temperature is required. Solutions of the combined pre-heating and flow control problem with the objective stated in Equation 4.15 and constraints in Equation 4.9 - Equation 4.14, Equation 4.16 and Equation 4.17 are investigated for a set of single WAs.

Firstly, we obtained Pareto fronts for two WAs with different duration to demonstrate possible trade-offs between the electricity consumption for water pre-heating $E_{e}\left(\Delta t_{\text {pre. }}\right)$ and user the flow discomfort $D_{\dot{m}_{\mathrm{d}}}$. The selected duration of the WAs is listed in Table 4.2 together with the comfortable tap water flow range $\left[\dot{m}_{\text {c.min }}, \dot{m}_{\text {c.max }}\right]$, user flow tolerance $\left[\Delta_{\text {tol }}^{-}, \Delta_{\text {tol }}^{+}\right]$and the user-desired tap water temperature ${ }^{5}$. Pareto solutions for two WAs with different duration can be found in Figure 4.19. The graphs represent the electricity consumption $E_{e}\left(\Delta t_{\text {pre. }}\right)$ as a function of the flow discomfort $D_{\dot{m}_{\mathrm{d}}}$. The flow discomfort is shown in percentage as a share of the maximum $D_{\dot{m}_{d}}$ for the certain WA. The color of each solution on Pareto front refers to a certain range of tap water flows and the time during which the user experiences flow discomfort $D_{\dot{m}_{d}}$. The color bar exhibits these values in the following format $\left[\dot{m}_{\mathrm{d}, \min }, \dot{m}_{\mathrm{d}, \max }\right], \Delta t_{D}$, which is the minimum and the maximum tap water flow values reached during the WA and the total duration of $D_{\dot{m}_{\mathrm{d}}}$. A number of the obtained tap water flow rates $\dot{m}_{\mathrm{d}}$ corresponding to the solutions on Pareto front for the 7-minute WA is presented in Figure 4.19(a).

\footnotetext{
${ }^{5}$ The selected values for simulations are base on the common sense considerations and publicly available statistics [118-120].
} 

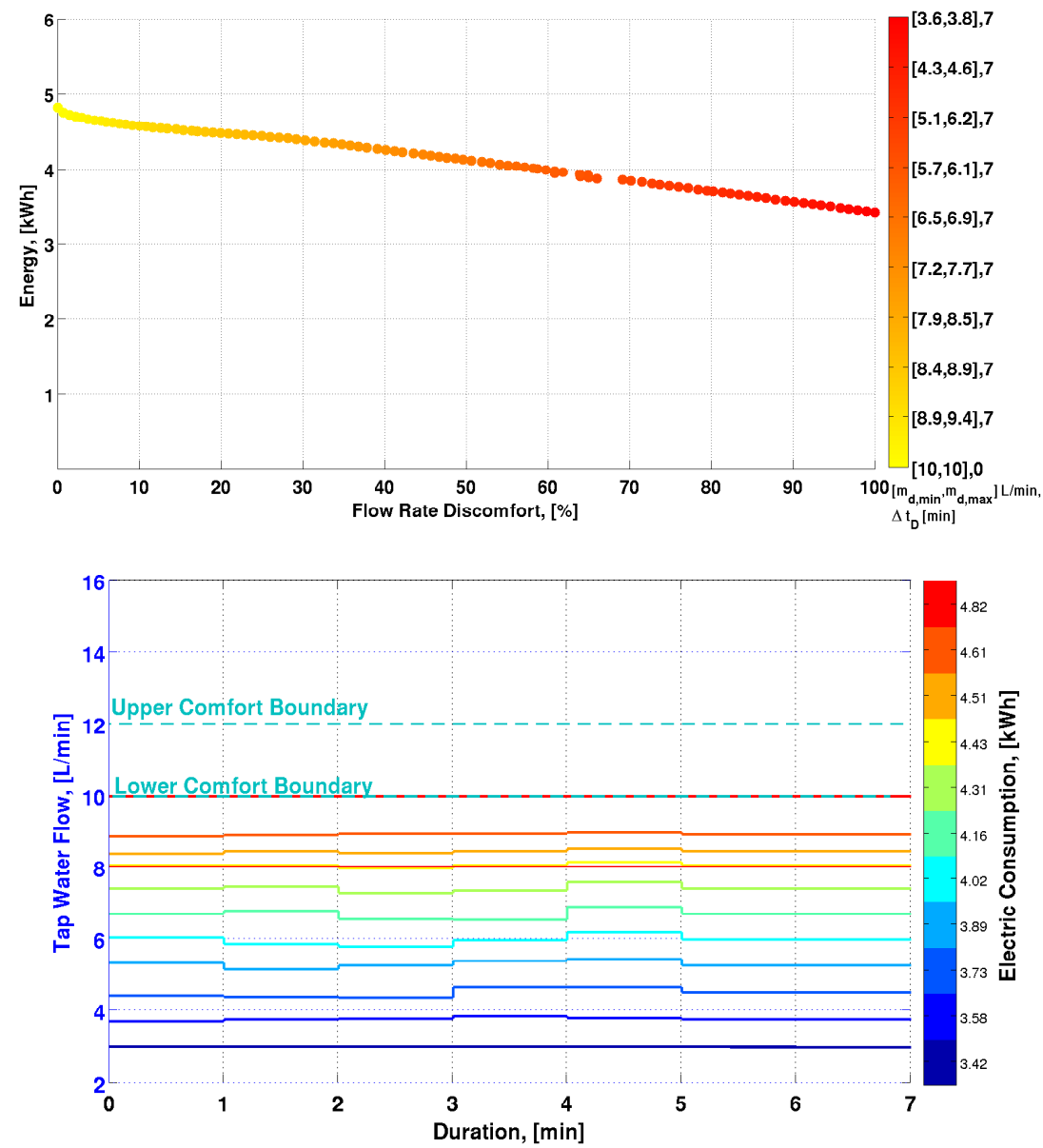

(a)

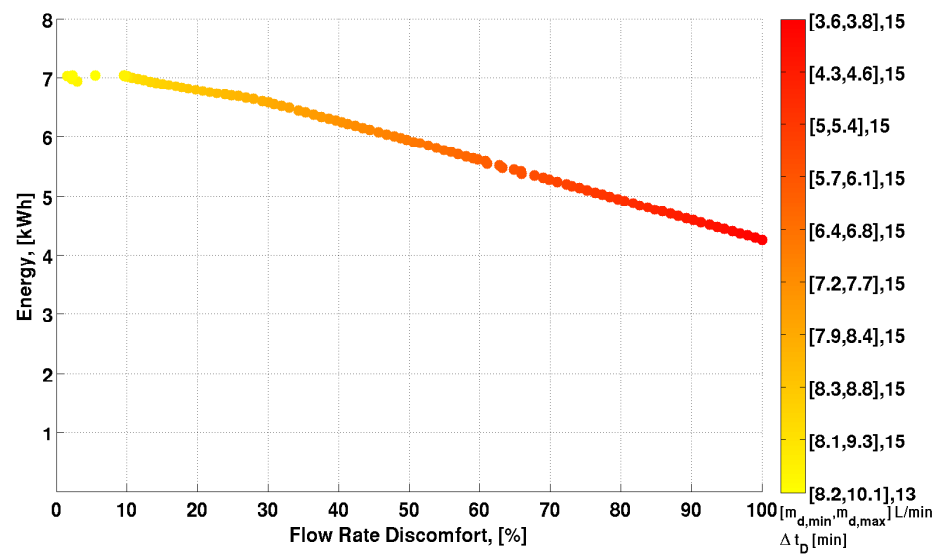

(b)

Figure 4.19: Pareto fronts for (a) 7-minute WA, (b) 15-minute WA. 
The optimization algorithm can lead to solutions with different values of $D_{\dot{m}_{d}}$ and equal levels of $E_{e}\left(\Delta t_{\text {pre. }}\right)$. Two of such solutions from Figure 4.19(b) are illustrated separately in Figure 4.20.
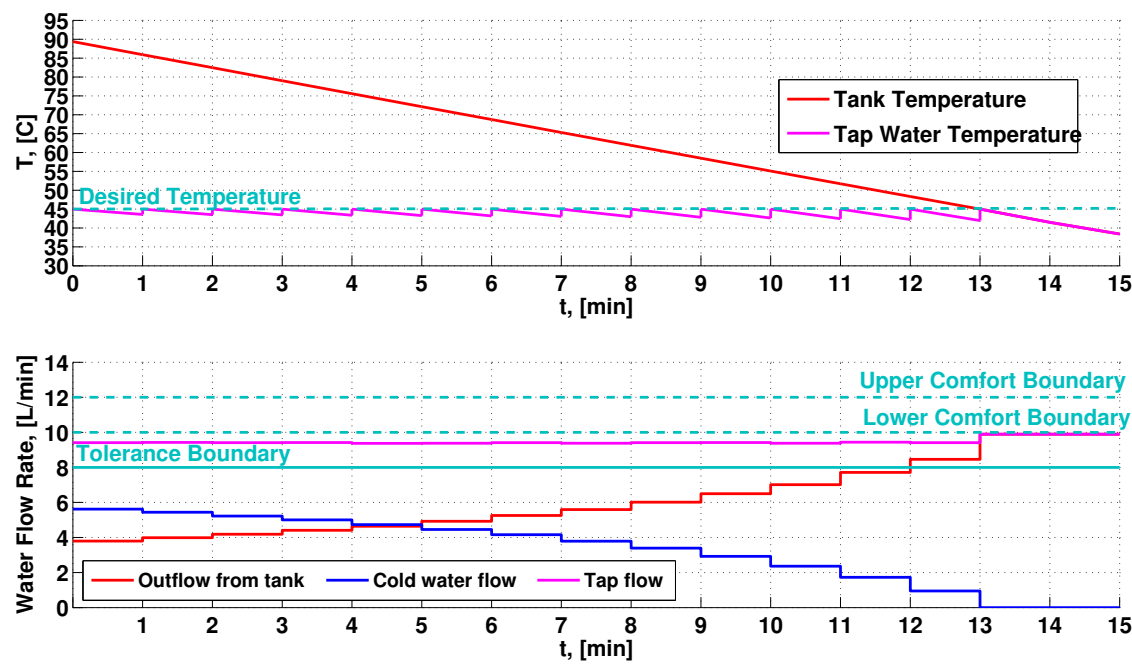

(a)

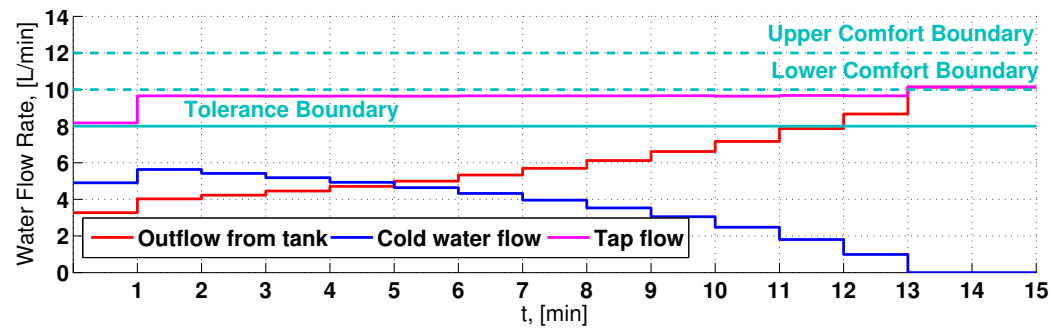

(b)

Figure 4.20: 15-minute WA \& fully charged tank under comfort degradation level of (a)

$$
D_{\dot{m}_{\mathrm{d}}}=10 \% \text {, (b) } D_{\dot{m}_{\mathrm{d}}}=3 \% \text {. }
$$

Furthermore, since the flow rate discomfort $D_{\dot{m}_{\mathrm{d}}}$ depends on the size $\Delta t$ of the control step (term $A_{\dot{m}_{\mathrm{d}, i}}$ in $D_{\dot{m}_{d}}$ ), we estimate the effect of altering $\Delta t$ on user flow discomfort $D_{\dot{m}_{\mathrm{d}}}$ and the electricity consumption for water pre-heating $E_{e}\left(\Delta t_{\text {pre. }}\right)$. The influence of the control step size on $D_{\dot{m}_{\mathrm{d}}}$ is demonstrated for two selected WAs in Figure 4.21 and Figure 4.22.

Not only affects the size of the control step the user flow discomfort $D_{\dot{m}_{\mathrm{d}}}$, but also impacts the user thermal discomfort $D_{\mathrm{T}}$, because bigger steps can lead to deeper drops of $T_{\mathrm{d}}(t)$. The thermal discomfort $D_{\mathrm{T}}$ was calculated for every solution on Pareto front of the considered WAs. The connection between the thermal discomfort $D_{\mathrm{T}}$ and the flow rate discomfort $D_{\dot{m}_{\mathrm{d}}}$ is represented for the 7-minute WA in Figure 4.23(a). The color of the curve corresponds to a certain tap water temperature range $\left[T_{\max }, T_{\min }\right]$ and total duration 


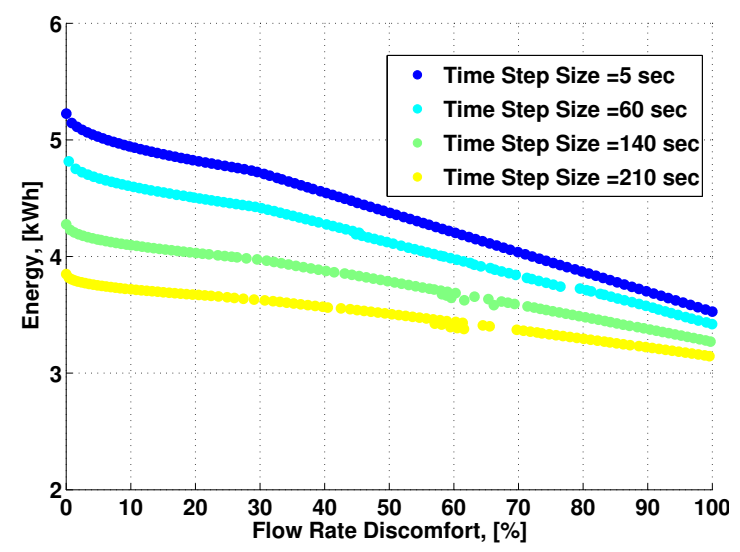

Figure 4.21: Varied size of timesteps 7-minute WA.

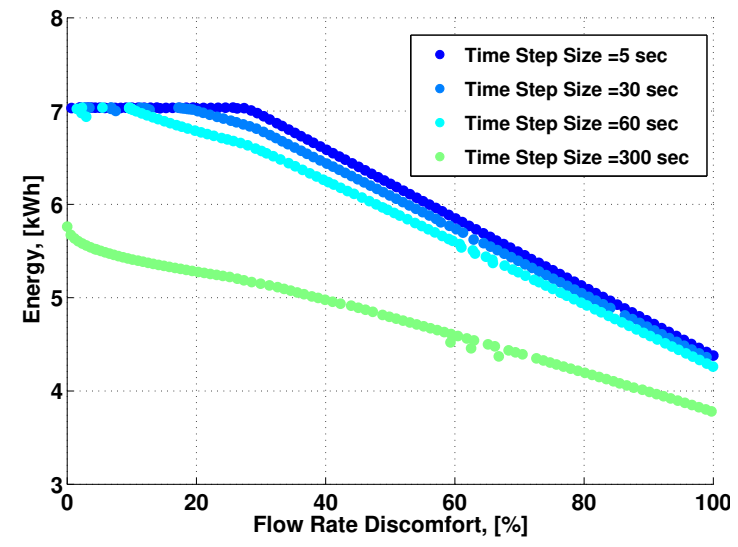

Figure 4.22: Varied size of timesteps 15-minute WA.

of both the flow and thermal discomfort $\Delta T_{\text {DIsc }}$ in the color bar. In addition, Figure 4.23(b) shows how the relation between $D_{\mathrm{T}}$ and $D_{\dot{m}_{d}}$ varies depending on the control step size.

\subsubsection{Results Discussion and Conclusion}

Based on Pareto solutions shown in Figure 4.19, one can see that the user can reduce $D_{\dot{m}_{\mathrm{d}}}$ at the cost of the increased $E_{e}\left(\Delta t_{\text {pre }}\right)$ and vice versa. This indicates a non-linear negative correlation between two functions. More exactly, it can be seen from Figure 4.19(a) that the higher the user flow comfort, the more electricity should be consumed during the water pre-heating under equal thermal comfort levels. This can be explained by the increase of the demanded energy due to the increased amount of water passing through the WH tank.

It is noteworthy that in case of the intensive hot water demand, the flow controller cannot handle the user request for a stable temperature during the whole WA. For example, the tap water temperature drops below the desired value within the last 2 minutes of the 


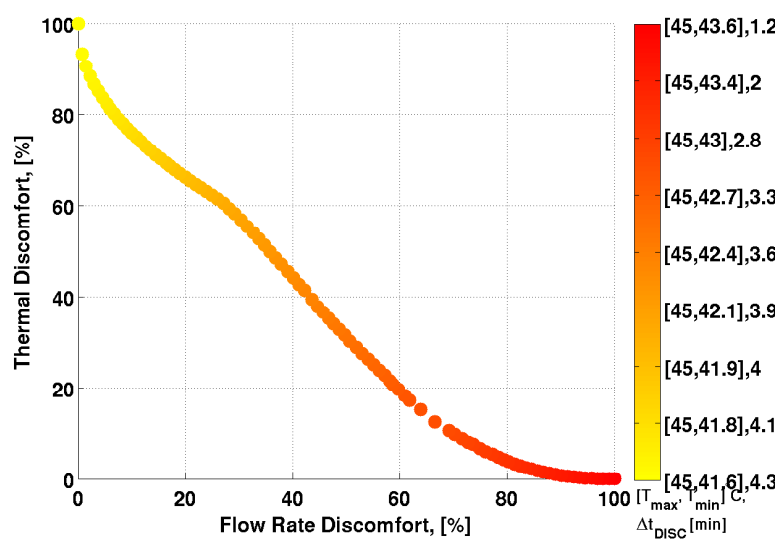

(a)

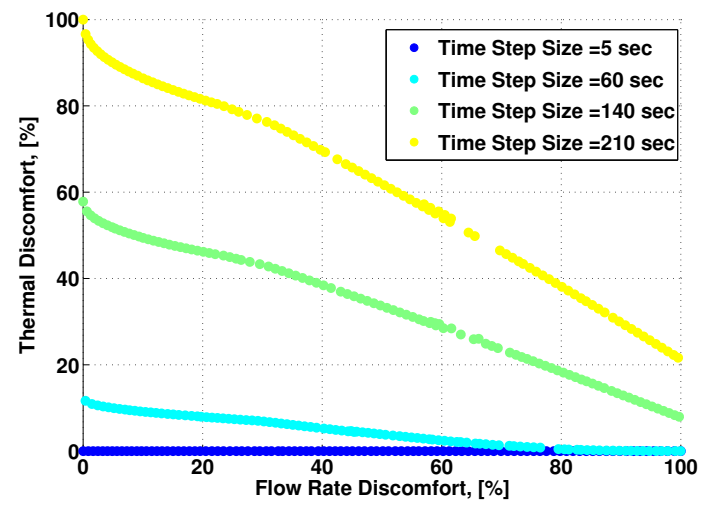

(b)

Figure 4.23: Effect on thermal discomfort for 7-minute WA.

15-minute WA as represented in Figure 4.20(a). Although the WH is heated up to the maximum safety allowed temperature $\left(90^{\circ} \mathrm{C}\right.$ in our case), the heat pre-stored in the $\mathrm{WH}$ tank is insufficient to provide the required water temperature of $45^{\circ} \mathrm{C}$ during the whole WA.

As it can be seen from Figure 4.19(b) the minimization of $D_{\dot{m}_{\mathrm{d}}}$ for long-lasting WAs can be achieved without the increase in the electricity consumption. A closer examination of two neighbor solutions on Pareto front shows that such a decrease of $D_{\dot{m}_{\mathrm{d}}}$ results in a steep jump of the tap water flow rate $\dot{m}_{\mathrm{d}}$ as can be seen from Figure 4.20. Such a sudden increase of the water flow may bring extra inconvenience to the user.

As it follows from Figure 4.21, bigger size of control step $\Delta t$ allows for minimizing $D_{\dot{m}_{\mathrm{d}}}$ while spending less electricity as compared to smaller steps. While the increase of the control step size has a positive effect on $D_{\dot{m}_{\mathrm{d}}}$ and $E_{e}\left(\Delta t_{\mathrm{pre}}\right)$, it has a negative effect on the thermal discomfort $D_{T}$ as shown in Figure 4.23(b). Bigger control steps make the tank water temperature drop to lower values leading to the increase of $D_{T}$. Considering the opposite effect of the step size on $D_{T}$ and $D_{\dot{m}_{\mathrm{d}}}$ the control step size could be another 
subject of optimization.

To summarize, the proposed control mechanism combines the water pre-heating strategy and the flow control strategy and allows for finding the best options for the water heating with respect to solutions of the flow control. Therefore, the combined control seems to be more efficient from the economical and user comfort perspectives than the decoupled operation of the water heating control and standard solutions for tap water temperature stabilization (such as TMVs).

\subsection{Conclusion}

In domestic hot water systems (HWSs) with insufficient heating capacities, users may experience inconvenient low water temperatures. This chapter tackles the problem of preserving the user thermal comfort in a HWS with an electric tank water heater (WH).

In this chapter, we considered two distinct scenarios of the hot water usage. These are a single hot water activity (WA) performed with and without the user thermal comfort tolerance. We assume that all the needed parameters of the expected WA are available from an ideal forecast. While in the first scenario the user desires to receive the tap water in the comfortable range of temperatures, a nearly stable tap water temperature is required in the second scenario. In regard to the two scenarios, we examined two control strategies in order to provide the user-desired level of the thermal comfort, namely the water pre-heating control and the combined flow and pre-heating control.

The idea of the pre-heating control is to heat the water upfront the WA only up to the temperature sufficient for the needed thermal comfort level. The best tank water temperature for the water pre-heating is found with respect to another control variable, the flow rate of hot water from the WH. In our approach, the tank water is pre-heated upfront the WA, after that the best water flows are established in the mixer tap and the WH is switched off. In other words, the user only sets the comfortable tap water flow at the WA start time, while the desired tap water temperature is automatically maintained by the controller of the mixer tap. The simulation results show that the pre-heating control is capable to attain multiple trade-offs between the objectives of minimizing the electricity consumption and minimizing the user thermal discomfort. It has been noticed that the initial tank water temperature at the beginning of the planning period has a big impact on the simulation results. For example, if the tank water temperature at the beginning of the planning horizon is equal to $50^{\circ} \mathrm{C}$, the electricity consumption for water pre-heating is reduced by about $66 \%$ as compared to the case with the initial water temperature of $15^{\circ} \mathrm{C}$. In the worst-case scenario where the $\mathrm{WH}$ is initially filled with cold water, the simulations of WAs with different intensity demonstrate an average reduction of the daily heat losses by up to $52 \%$ and the maximum decrease of the daily electricity consumption by $10 \%$ as compared to the conventional deadband control under (approximately) the same thermal comfort levels. The real daily gain depends on the initial tank water temperature, insulation of the $\mathrm{WH}$ and parameters of the expected WA. It has been found that in the worst-case scenario the pre-heating control overplays the deadband control in daily electricity consumption levels in case of (a) intense hot water demand (long-lasting WAs and WAs with big tap water flow rates), and (b) poor insulation of the WH (the energy consumption improved by roughly $2-3 \%$ ).

Based upon the flow control principle of thermostatic mixing valves, we proposed a 
combined flow control and pre-heating control mechanism for a scenario where a nearly stable tap water is desirable by the user. The distinguishing feature of the proposed control is that water heating control is performed with respect to solutions of the flow control, i.e. two types of control are coupled together. More specifically, the flow control algorithm finds the best values of the hot and cold water flow rates to be maintained during a WA in a step-wise manner and the best tank water temperature value to established by the water preheating control at the beginning of the WA. Unlike the previous scenario of the hot water usage, the controller at the tapping point can change both the water inflows and outflow in the mixer tap during the entire WA. The simulation results of the combined control show its potential to support a close to stable tap water temperature during WAs while minimizing the heat losses and user flow discomfort. The results match our expectation that the decreased tap water flow rate can support the user thermal comfort. Furthermore, it has been noticed that the size of the control step of the flow control component has an opposite effect on the user flow and thermal comfort.

To summarize, the proposed control mechanisms allow for balancing between different objectives such as maximization of energy/money savings and minimization of the end-user comfort violations in distinct scenarios of the hot water consumption. In addition, the combined flow and pre-heating control can potentially support the objective of water savings by managing the amount of water passing through the mixer tap. 



\section{5 \\ CONTROL STRATEGIES FOR Multiple Water Activities}

This chapter focuses on efficient control of domestic electric tank water heaters (WHs) in a scenario of multiple hot water activities (WAs). We specifically tackle the limitations of the conventional deadband control that are the energy inefficiency due to stand-by heat losses, comfort inefficiency caused by fixed thermostat setpoints and incapability to shift the WH load in response to variable prices.

To overcome these shortcomings, we propose a new control mechanism for WHs based on two optimization models, namely the energy and price models. Both of the models allow for planning of hot water production in the WH taking into account different objectives.

The energy model aims to increase the energy efficiency of the WH by reducing the stand-by heat losses while maintaining the required user thermal comfort and by aligning the hot water supply to the minimal, yet comfortable, level.

The price model, on the other hand, pursues the goal of money savings for water heating by shifting the WH load to low-price periods with regard to the user comfort needs.

The distinguishing feature of the proposed control mechanism is that it accounts for presence of mixer taps in a hot water system and controls the hot water supply. The efficiency of the control solutions is evaluated based on a series of simulations.

\footnotetext{
$A$ discussed in the previous chapter, in the conventional deadband control of electric $\mathrm{AS}$ tank water heaters (WHs), the maximum amount of heat stored in tank is predefined by thermostat setpoints. This can be risky, if the actual demand exceeds the available amount of thermal energy. Next to the risk of user discomfort, the deadband control suffers from extensive heat losses during the periods of no hot water usage, so-called stand-by losses, and thus it lacks energy efficiency. A better solution is to heat the water in compliance with the expected hot water demand, i.e. to prepare exactly as much heat as needed in the WH tank right upfront the actual usage. In this case, the providence of
} 
the user-desired thermal comfort is accompanied by minimum heat losses which is more efficient from the economical viewpoint.

Energy suppliers have been traditionally offering flat electricity tariffs to residential consumers, where electricity rates do not vary with energy usage or the time. Today, energy companies provide customers with time-varying retail energy prices through timeof-use, critical-peak and real-time pricing tariffs. Such tariffs foster lower energy usage during the periods of high electricity prices and stimulate electricity usage during the low-price periods, which can, amongst others, diminish the peaks of energy demand in the electricity network. Consumers benefit financially in return of shifting their electricity consumption to low-price periods. However, a big alteration in the electricity consumption of WHs due to the load shifting might bring inconvenience to occupants, if not exercised cautiously with respect to user comfort needs. Note that the traditional deadband control does not explicitly account for price signals. A user informed of the intra-day market prices may want to manually increase the thermostat setpoints during the low-price periods and attempt to lower them when the prices are high. However, a regular user is unaware about the best thermostat settings that will deliver the maximum profits and provide a decent level of thermal comfort.

Two control strategies introduced in Chapter 4, the water pre-heating and flow control, are capable to prepare and provide the hot water with regard to the expected user thermal comfort and constraints of the WH. While the pre-heating control allows for building up the heat in the amounts exactly required by the user, the flow control can provide the tap water at the lowest, yet comfortable for the end-user temperature. Released from the necessity to always keep the tank water hot, the pre-heating control stands in line with the goal of the reduction of heat losses. Besides, by executing the water pre-heating during the low-price periods, one can shift the WH load and save money. The flow control can contribute to the energy savings in case of the user thermal tolerance. In that regard, the outlined strategies combined together can provide a more flexible control of the $\mathrm{WH}$ than the conventional deadband control both in terms of the user comfort satisfaction and energy/money savings. However, the control mechanism proposed in Chapter 4 is suitable only for the case of a single WA.

In general, the household hot water consumption is given by multiple WAs with possibly different duration, water flow rates and diverse user comfort preferences associated with them. Moreover, WAs may overlap in time when multiple tapping points are used in parallel. Therefore, a different control mechanism than the suggested combination of the pre-heating and flow control is needed for the household water heating.

For the scenario where multiple WAs can occur during a day, we tackle the following limitations of the deadband control in this chapter:

- inefficient user comfort management;

- energy inefficiency originating from the stand-by heat losses;

- incapability to respond to variable electricity prices.

To overcome the above downsides of the deadband control of WHs, we propose a new control mechanism based on two optimization models, namely the energy model and the price model. These models are used separately for solving distinct problems: 
- the energy model aims to minimize the daily heat losses while satisfying the required user thermal comfort (or the "energy-comfort" problem);

- the price model seeks to minimize the daily water heating costs under the given requirements for the user thermal comfort (or the "money-comfort" problem).

Because the objective function of the energy optimization model is the minimization of the heat losses, one can expect a reduction of the daily electricity consumption of the $\mathrm{WH}$, i.e. the energy model can lead to the electricity savings as compared to the deadband control. On the other side, the price optimization model aims to minimize the heating costs, thereby the model can bring money savings in comparison with the deadband control. Since two models pursue different optimization goals, they are applied separately from each other. Specifically, the price model is only applicable for the case of variable electricity prices, while the energy model can be used regardless to the electricity tariff structure.

The tailored models are built upon the principles of the water pre-heating and flow control examined in Chapter 4. Recap, the solution of the pre-heating control for a single WA with user thermal tolerance consisted of two values $\left\{T^{*}, \dot{m}^{*}\right\}$ that defined the best values of the tank water temperature $T^{*}$ and the hot water outflow from the $\mathrm{WH} \dot{m}^{*}$ in terms of the minimum user comfort disruptions and the minimum heat losses. In case of multiple WAs during a day, these variables should be defined for every WA. A naive approach would be to solve this problem independently for every WA. However, in that case the best solution $X_{i}^{*}=\left\{T_{i}^{*}, \dot{m}_{i}^{*}\right\}$ for the WA with index $i$ might never be fulfilled due to the hot water demand of the preceding WA $i-1$, the time span between the WAs and technical constraints of the WH. For example, the time between two WAs with indices $i-1$ and $i$ is too short to heat the water up to the required temperature value $T_{i}^{*}$. It is apparent that the solution for a given WA should be found with respect to the previous solutions.

We approach this problem by breaking the daily timescale into $N$ even time intervals and solve a set of overlapping sub-problems on all the intervals. The basic decisions on every time interval are (i) to heat or not to heat the water $x_{i} \in\{0,1\}, i \in[1, N]$ and (ii) the hot water outflow from the WH $\dot{m}_{i}, i \in[1, N]$. The variables controlled by the proposed mechanism are shown schematically in Figure 5.1.

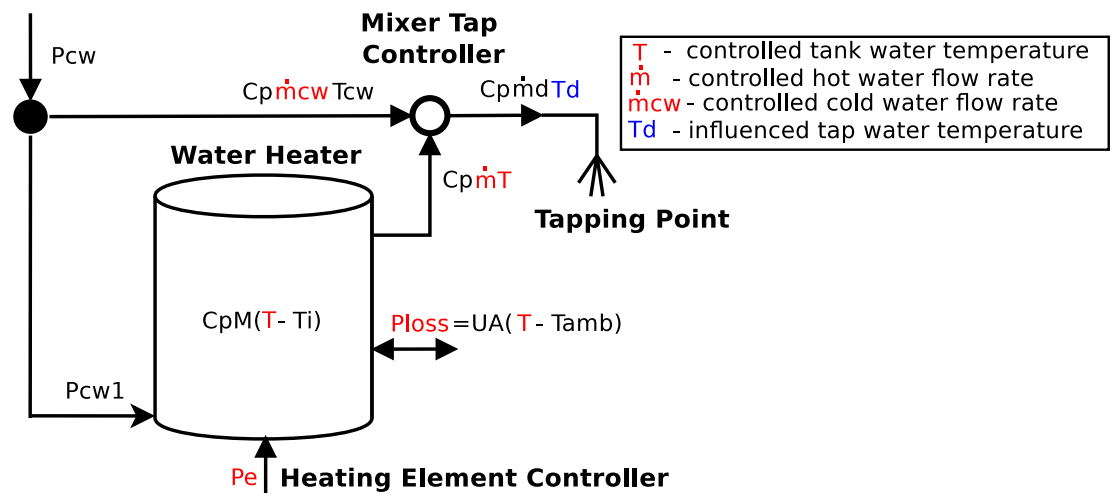

Figure 5.1: Control variables. 
In our approach, the decision variables on a given time interval are influenced by the user comfort needs accounted for by the constraints of the models and by the objective to minimize (a) the heat losses for the energy model, or (b) the water heating costs in case of the price model. Furthermore, the decisions are made not only in compliance with a comfort requirement and objective function value for the current interval, but also considering the impact of the decision on the user comfort and objective values on all coming intervals.

The distinguishing features of the devised energy and price models can be summarized as follows. Both of the tailored models are formulated as discrete-time Linear Programming optimization problems. The models account for the thermodynamics of the hot water system that consists of the WH unit and (possibly) multiple mixer taps connected by a system of pipes. The tank water temperature take values on a continuous scale ranging from the cold water temperature to the maximum safety allowed temperature $\left[T_{\mathrm{cw}}, T_{\mathrm{wh} \text { max }}\right]$. In our approach, we control the hot water production by controlling the states of the heating elements of the WH $x_{i}, i \in[1, N]$ on the side of the WH. In addition to the hot water production, we control the hot water supply by controlling the hot water flow rate $\dot{m}_{i}, i \in[1, N]$ in the mixer tap(s) (the controller(s) of the mixer taps in Figure 5.1). The values $x_{i}, i \in[1, N]$ are found in agreement with the values $\dot{m}_{i}, i \in[1, N]$ by the energy and price optimization models. Therefore, the water heating control is coupled to the control of the water flows in the mixer tap. The models utilize the time- and comfort-related information about the expected WAs available from an ideal forecast. The user thermal comfort needs are quantified by means of the user thermal comfort model presented in Chapter 3 . The information about the WH thermodynamics and WAs including user thermal comfort preferences is organized into matrices that serve as input to the models.

The output of the models is a schedule of binary states of the WH (on/off) and a schedule of the tank water temperatures on a discrete timescale that (a) minimize the daily electricity consumption in case of the energy model and (b) minimize the daily heating costs in case of the price model, while satisfying the user thermal comfort requirements on each time interval. To be exact, the solution of each of the models consists of a pair of vectors $\mathbf{x}=\left\{x_{i} \mid x_{i} \in\{0,1\}, i \in[1, N]\right\}_{1 \times N}$ and $\mathbf{T}=\left\{T_{i} \mid T_{i} \in\left[T_{\text {cw }}, T_{\text {wh } \max }\right], i \in[1, N]\right\}_{1 \mathrm{x} N}$. The binary vector $\mathrm{x}$ represents the states of the heating elements, i.e. $x_{i}$ is a decision to either switch the $\mathrm{WH}$ on or off on the $i$-th interval of the timescale $i=[1, N]$. The vector $\mathrm{T}$ contains the tank water temperature values at the end of the $i$-th interval, if the solution $x_{i}$ is implemented. It is important to note that the values in $\mathrm{T}$ incorporate the information about the best values of the hot water outflows from the WH $\dot{m}_{i}, i \in[1, N]$ found implicitly by the proposed models, i.e. the hot water flow rates are not directly returned by the models and have to be retrieved based on the found values of $\mathbf{x}$ and $\mathrm{T}$. By using the thermodynamic model of the $\mathrm{WH}$, the control mechanism restores the vector of the best hot water outflows from the $\mathrm{WH} \dot{\mathbf{m}}=\left\{\dot{m}_{i} \mid \dot{m}_{i} \in\left[0, \dot{m}_{\mathrm{d}, i}\right], i \in[1, N]\right\}_{1 \mathrm{x} N}$ associated with the values in $\mathbf{x}$ and $\mathrm{T}$. The value $\dot{m}_{i}=0$ corresponds to no hot water usage and $\dot{m}_{i}=\dot{m}_{\mathrm{d}, i}$ refers to the situation where all the tap water is withdrawn from the WH tank during the $i$-th interval of the timescale.

We evaluate the performance of the proposed two models based on a series of simulations for a single day, multiple days and WAs overlapping in time, i.e. when multiple tapping points are used in parallel. 


\subsection{Residential Hot Water Consumption}

The residential hot water consumption has a stochastic character. Occupants perform daily hot water activities (WAs) such as washing hands, taking a shower, etc. at random times based on their individual preferences.

The time-related parameters of WAs, the starting times and duration, can, in general, vary across occupants as well as for a single person. A household hot water usage profile can consist of frequent consecutive WAs next to the WAs occurring relatively distant in time from each other. Additionally, the user can turn the tap on and off multiple times during the same WA. In multi-tenant households, residents can also perform more than one WA at the same time. Furthermore, domestic hot water consumption ordinarily exhibits a pattern of morning and evening peaks during the working days. These peaks correlate with space occupancy periods that usually extend during the weekends. As a result, the hot water usage periods are typically more spread along the day in the weekends than in the working days.

Besides the time-related parameters of WAs, other relevant parameters of WAs include the tap water temperature and the tap water flow rate. These parameters determine the thermal power demand during the hot water usage. People typically desire the tap water temperature and the tap water flow to be within some comfortable ranges that may vary even for a single person. Depending on the type of the WA performed (e.g., hygiene or cleaning) and physiology of a human body (skin sensitivity) some WAs can be fulfilled in a relatively wide range of tap water temperatures and tap water flow rates, while other WAs can be accomplished only in narrow ranges.

Water activities with big hot water demand are not rare in households. According to official reports [121], WAs such as taking a shower and bathing represented $33 \%$ of the estimated domestic hot water usage in EU-28 in 2012. The statistics of showering show that users commonly request relatively high tap water flow rates (between 7.5 and 14 $[\mathrm{L} / \mathrm{min}][122,123])$ with tap water temperature ranging between 36 and $42\left[{ }^{\circ} \mathrm{C}\right]$ [121]). A typical showering WA takes 7-8 minutes in average with a daily frequency per user from 0.6 and 1.5 (in average once per day). The estimates show that showering can consume up to 1100.93 [kWh] of energy per person annually. The average yearly electricity demand associated with showering is 4.13 times higher than that for washbasin taps, although people tend to use taps about 7 times more often during the working days and 10 times more often in the weekends.

\subsection{Limitations of Conventional Deadband Control of WATER Heaters}

The fixed thermostat setpoints in the conventional deadband control of tank water heaters (WHs) impede the efficient management of the user comfort as we have investigated in Chapter 4. Apart from a potential negative impact on the user comfort, the deadband control consumes the electricity also in stand-by periods which makes it energy inefficient. In the following, we consider the user comfort and energy related properties of the deadband control next to each other.

Once manually set, the thermostat setpoints usually remain unchanged during a day in the deadband control, i.e. they are fixed. In case the chosen setpoints are too low, there is 
a risk that a user might experience uncomfortable cold water, as for example during an unforeseen intense hot water demand or within the periods of frequent hot water activities (WAs). Along with this, raising the upper thermostat setpoint leads to the higher heat losses. Besides, in specific scenarios of hot water usage, the user might want to get a stable tap water temperature. Moreover, as we discussed in Section 4.5.1, standard solutions for the tap water temperature stabilization such as thermostatic mixing valves (TMVs) operate independently from the deadband control, i.e. their operation is not accounted for in the WH control, which is not optimal from the economical and user comfort points of view. Therefore, the deadband control does not suit all possible scenarios of household hot water consumption comfort wise.

Another downside of the deadband control is related to its energy inefficiency. The fixed setpoint temperatures help ensure accessibility of hot water on user demand, which, in general, increases occupants' comfortability. However, such operation brings superfluous stand-by heat losses during the periods when the hot water is not used (e.g., during the night). As a result, the WH consumes electricity for maintaining the tank water temperature within the thermostat setpoints, although the hot water is not really needed for occupants. The resulting stand-by electricity consumption is determined by the heat losses and depends on the thermostat setpoints, duration of stand-by periods, the insulation of the $\mathrm{WH}$ and the ambient temperature.

With a widespread adoption of Demand Response, the possibility to shift the electricity consumption of WHs in response to variable price signals from the grid is becoming of high importance. Unfortunately, the conventional deadband control of WHs does not take into account the information about the electricity prices, thus cannot automatically shift the WH electricity consumption outside the periods of high prices. In that regard, one may choose to manually adjust the thermostat setpoints during the deadband control. By raising the $\mathrm{WH}$ thermostat setpoints during the low-price periods and by decreasing them when electricity rate is high, the water heating periods and, therefore, the $\mathrm{WH}$ electricity consumption can be aligned to the periods of low electricity prices and lead to money savings (as compared to the deadband control). However, it is hard for a regular user to define the appropriate thermostat settings without considering the WH technical characteristics (e.g., the relationship between the tank water temperature and the electricity consumption), the expected hot water demand and possible implications of such actions on the end-user comfort, i.e. the user is unaware about the best thermostat settings that will deliver the maximum money profits and maintain the needed level of thermal comfort. Furthermore, the best times of such thermostat adjustments are unknown to the user. Finally, the user might need to regulate the setpoints multiple times during a day, since the intra-day market prices can vary. This means that the manual thermostat tunning does not seem to be a feasible solution for shifting the WH electricity consumption.

To summarize, the limitations of the conventional deadband control of WHs entail:

1. inefficient user thermal comfort management caused by:

(a) fixed (static) thermostat setpoints;

(b) decoupled operation of TMVs from the deadband control in scenarios where a stable tap water temperature is required; 
2. energy inefficiency resulting from the unneeded electricity consumption during the stand-by periods;

3. impossibility to shift the WH electricity consumption in response to variable electricity prices.

In this chapter, we propose a new control mechanism for domestic WHs to overcome the above limitations of the conventional deadband control.

\subsection{Organization of Chapter}

We tackle three problems of the conventional deadband control outlined in Section 5.2 in a scenario where multiple hot water activities (WAs) occur during a day.

We start with a description of specifics of Problems 1 and 2 in case of multiple WAs. Next, we formulate an energy-comfort problem and propose a solution to that problem in the form of an energy optimization model, the "energy model". The aim of the energy-comfort problem is to minimize the electricity consumption of the $\mathrm{WH}$ while providing the required level of the user thermal comfort.

Finally, we describe our approach to Problem 3 where we shift the WH electricity consumption in response to variable electricity prices. In that regard, we formulate the money-comfort problem and solve it by means of a price optimization model, the "price model". Solving the money-comfort problem enables money savings for water heating while respecting the user thermal comfort.

\subsection{ENERGY MODEL}

\subsubsection{Preliminary Discussion and Problem Formulation}

Inefficient handling of user comfort together with inefficient energy consumption outlined in Section 5.2 undermine the efficiency of the conventional deadband control. In this section, we elaborate on Problems 1 and 2 of the conventional deadband control considering a scenario where multiple WAs during a day. After that we formulate the energy-comfort problem.

Consider a simplified example where two WAs non-overlapping in time take place during a day. Suppose, the information about the time-related and comfort-related parameters of the WAs is known from an ideal forecast, and the user can tolerate the tap water temperature in some comfortable range of temperatures. Let the start times of the first WA with index $i=1$ and the second WA with index $i=2$ be $t_{\mathrm{str} 1}$ and $t_{\mathrm{srt} 2}$, respectively. Assume that the WAs have equal duration $\Delta t,\left(t_{\mathrm{str} 1}+\Delta t \leq t_{\mathrm{str} 2}\right)$ and the tank water is initially cold $T(0)=T_{\mathrm{cw}}$. Furthermore, assume that the comfort-related parameters of the WAs are identical and given in the form of the comfortable range of the tap water temperature values $\left[T_{\text {c.min }}, T_{\text {c.max }}\right]$ and the fixed tap water flow rate $\dot{m}_{\mathrm{d}}$. Moreover, in this example the user sets only the comfortable tap water flow $\dot{m}_{\mathrm{d}}$ at the beginning of each WA, while the initial tap water temperatures are established automatically by the controller of the mixer tap that sets the mixing proportions $k_{1}=\frac{\dot{m}_{1}}{\dot{m}_{\mathrm{cw}, 1}}=\frac{\dot{m}_{1}}{\dot{m}_{\mathrm{d}}-\dot{m}_{1}}, \dot{m}_{\mathrm{d}}=$ const and $k_{2}=\frac{\dot{m}_{2}}{\dot{m}_{\mathrm{d}}-\dot{m}_{2}}$ at times $t_{\mathrm{str} 1}$ and $t_{\mathrm{srt} 2}$, respectively. The task is to find the best heating periods (best times to switch on/off the $\mathrm{WH}$ ) and the best mixing proportions $k_{1}$ and $k_{2}$ (or simply the hot 


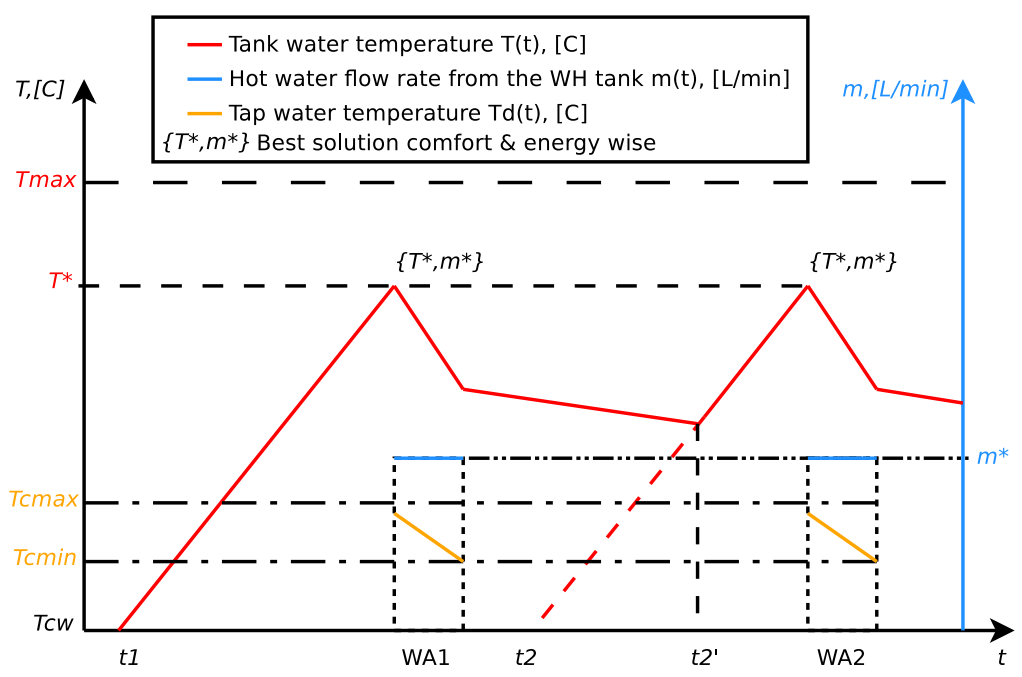

Figure 5.2: Simple concatenation of heating solutions.

water flow rates $\dot{m}_{1}$ and $\dot{m}_{2}$ ) for the mixer tap that provide (a) zero thermal discomfort to the user (full comfort), (b) the minimum heat losses from the WH tank.

A naive approach could be to solve the pre-heating problem in Equation 4.4 (Chapter 4) independently for each of the WAs (without considering the presence of another WA) and find the best solutions $\left\{T^{*}, \dot{m}^{*}, t_{1}^{*}\right\}$ and $\left\{T^{*}, \dot{m}^{*}, t_{2}^{*}\right\}$ in terms of the minimal user thermal discomfort and the minimal heat losses. These solutions represent the best tank water temperature value $T^{*}$ to be established at the start times of the WAs, the hot water flow rate from the WH tank $\dot{m}$ to be maintained during the WAs and the starting times $t_{1}^{*}$ and $t_{2}^{*}$ to initiate the water pre-heating upfront the WAs. Firstly, in this case the decision maker has to decide beforehand whether or not to keep the WH switched on during the WAs. Secondly, considering the timing of WAs and technical constraints of the WH the real solution to the problem can change depending in the following cases:

1. the start time of the heating period for the second WA $t_{2}^{*} \geq t_{\operatorname{str} 1}+\Delta t$;

2. the start time of the heating period $t_{2}^{*}<t_{\mathrm{str} 1}+\Delta t$.

In the first case, the heating periods can be obtained by a simple concatenation of the tank water temperature curves obtained for WAs separately as shown in Figure 5.2. The heating period for the first WA, the values of $T^{*}$ and $\dot{m}^{*}$ remains the same. While and the start-time for water heating for the second WA becomes $t_{2}^{\prime}$.

In the second case, the tank water temperature value at the end of the first WA is below the temperature curve of the pre-heating period for the second WA (obtained separately) as shown in Figure 5.3. Since the real temperature value at the start of the water heating for the second WA is lower by $\Delta T$ than the found one, the user thermal comfort during the second WA will be deteriorated. To prevent the user thermal discomfort, the water can be heated to a higher temperature $T_{1}^{*}>T^{*}$ prior to the first WA. As a result, the tap water temperature $T_{\mathrm{d}}(t)$ will increase during the first WA and the user will experience the 


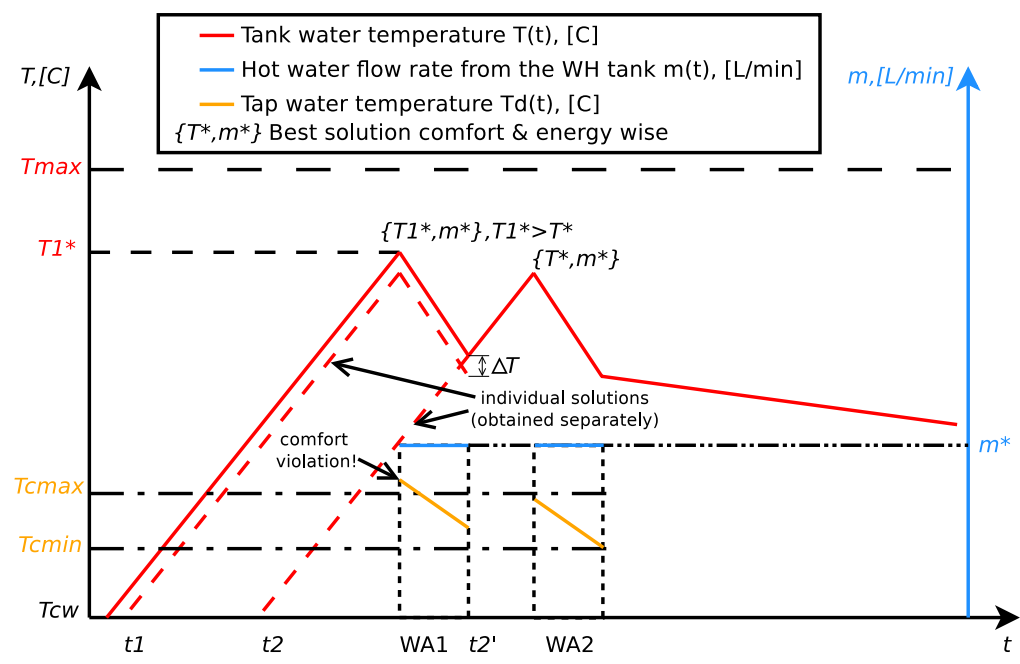

Figure 5.3: User comfort violation during first WA.

uncomfortably high temperature $T_{\mathrm{d}}\left(t_{\mathrm{str} 1}\right)>T_{\text {c.max }}$, if the hot water flow rate $\dot{m}_{1}^{*}=\dot{m}^{*}$ is not changed accordingly.

Based on Equation 2.7, the new solution for the first WA besides $T_{1}^{*}>T^{*}$ should also have the reduced hot water flow $\dot{m}_{1}^{*}<\dot{m}^{*}$ so that $T_{\text {c.min }} \leq T_{\mathrm{d}}\left(t_{\text {str } 1}\right) \leq T_{\text {c.max }}$. As a matter of fact, there may be multiple solutions for hot water flow rate that satisfy the user thermal comfort during the first WA as depicted in Figure 5.4. Figure 5.4 illustrates two of such solutions $\dot{m}_{1}^{*}<\dot{m}^{*}$ and $\widetilde{\dot{m}}_{1}^{*}<\dot{m}^{*}$ (shown in green and violet). Any of these two solutions $\left\{T_{1}^{*}, \dot{m}_{1}^{*}\right\}$ or $\left\{T_{1}^{*}, \widetilde{\dot{m}}_{1}^{*}\right\}$ changes the tank water temperature curve $T(t)$ during the first WA. Consequently, the new tank water temperature values $T\left(t_{2}^{\prime}\right)$ and $\widetilde{T}\left(t_{2}^{\prime}\right)$ at the end of the first WA will become above the old tank water temperature value. Which also means that heating the water during the period $\left[t_{2}^{\prime}, t_{\text {str } 2}\right]$ for the second WA starting from the new temperatures $T\left(t_{2}^{\prime}\right)$ and $\widetilde{T}\left(t_{2}^{\prime}\right)$ will lead to higher water temperatures at the start of the second WA than the best found value $T^{*}$, which is not the best alternative from the economical and user comfort points of view.

Based on the above examples, we can conclude that solutions for the first and second WAs should be found in agreement with each other.

In general, finding the best solutions that provide satisfactory user comfort at the minimum electricity consumption in case of multiple WAs differs from finding solutions for a single WA. Since all WAs are connected by a single tank water temperature transition process, preceding WAs can influence the following WAs. The degree of influence depends on the initial tank water temperature, the time lags between WAs, time-related and comfortrelated parameters of WAs, technical characteristics of the WH and the ambient temperature. In a scenario of multiple WAs, the problem unfolds as searching the best times for water heating and hot water flow rates from the WH considering all the WAs present during a day.

Summarizing, the energy-comfort problem can be formulated as follows. 


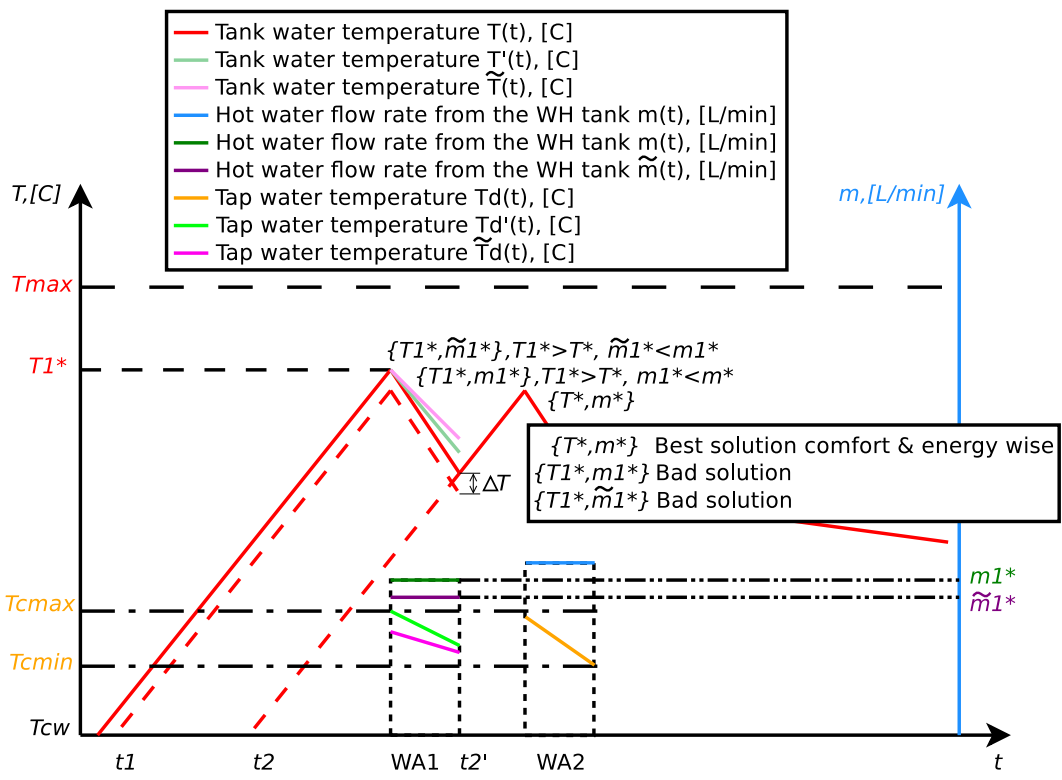

Figure 5.4: User comfort violation during second WA.

\section{ENERGY-COMFORT PROBLEM}

Given a WH with certain engineering characteristics and a daily profile of

hot water activities with associated user thermal comfort preferences,

find the best schedule of water heating periods and hot water flow rates that

minimize the daily electricity consumption, while satisfying the required user comfort.

\subsubsection{OUR Approach}

The most straightforward measure for reducing the electricity consumption is to reduce the stand-by heat losses. In that respect, it seems meaningful to keep the tank water at lower temperatures. This measure can, however, negatively impact the user thermal comfort due to the cooler water temperatures.

We therefore propose to keep the water cold in periods of no hot water usage and preheat the water right upfront WAs and only up to the temperatures that exactly fulfill the user thermal comfort needs, i.e. without overheating. In our approach, we achieve lower tank water temperatures by shutting the heating elements of the $\mathrm{WH}$ off, and switching them on again prior to WAs in order to ensure the user-desired thermal comfort.

Additionally, we propose to provide the tap water at the minimal, yet comfortable to the end-user, temperature during WAs. More precisely, the closer the curve $T_{\mathrm{d}}(t)$ is to the Lower Comfort Boundary $T_{\text {c.min }}$ during the WA, the smaller is the thermal energy $E_{\mathrm{d}}(t)$ delivered to the user and consequently the lower is the electricity consumption (based on the energy conservation law). Therefore, the existance of the user thermal tolerance can be 


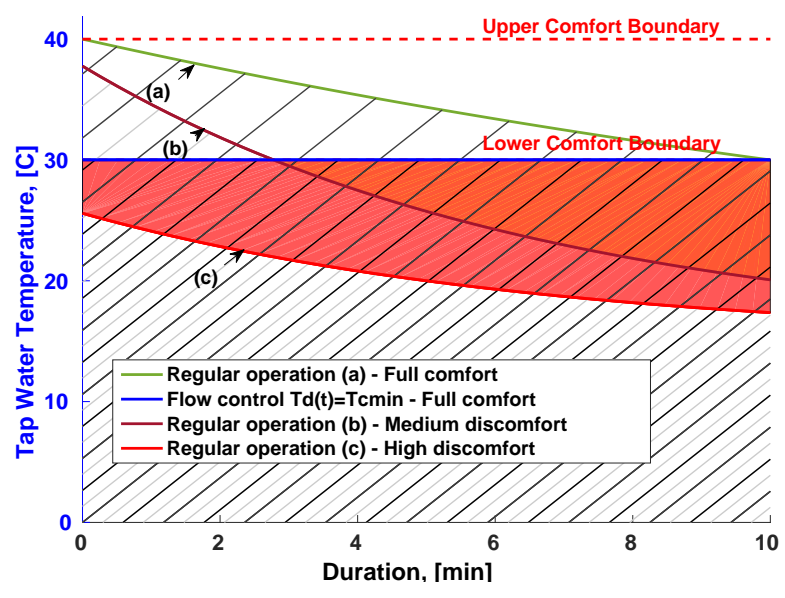

Figure 5.5: Water activity with temperature tolerance.

seen as an enabler of additional reductions of the electricity consumption. Unlike the heat losses, the controllability (or flexibility of decreasing) of $E_{\mathrm{d}}(t)$ is, however, limited. This is mainly due to psychophysiological abilities and readiness of users to tolerate the tap water temperature drops, which might be not present.

In case the user thermal tolerance is present, we provide the tap water temperature at the Lower Comfort Boundary by means of the flow control in our approach. To illustrate, let us consider a 10-minute WA performed with the tap water flow rate $\dot{m}_{\mathrm{d}}=12[\mathrm{~L} / \mathrm{min}]$ and some user thermal tolerance illustrated in Figure 5.5. The user thermal comfort zone is restricted by the Lower Comfort Boundary $T_{\mathrm{c} \text { min }}=30^{\circ} \mathrm{C}$ and the Upper Comfort Boundary $T_{\mathrm{c} \max }=40^{\circ} \mathrm{C}$. This means that any tap water temperature between these comfort boundaries causes zero thermal discomfort to the end-user, while any temperature outside the comfort zone is considered as uncomfortable ${ }^{1}$. The solid regions in Figure 5.5 graphically represent the user thermal discomfort in case of the tap water temperature (b) and (c).

Mixing taps are traditionally controlled by manually setting the desired tap water flow rate $\dot{m}_{\mathrm{d}}(t)$ and proportion of the hot and cold water flows $k=\frac{\dot{m}(t)}{\dot{m}_{\mathrm{cw}}(t)}$ at the beginning of a WA $t=0$. Suppose, the user does not re-adjust the mixing proportion $k$ during the WA, meaning that the tap water flow remains constant during the WA $\dot{m}_{\mathrm{d}}(t)=$ const, $t \in\left[0, \Delta t_{\mathrm{WA}}\right]$. Then, the tap water temperature $T_{\mathrm{d}}(t)$ gradually declines with the progress of the WA, as discussed in Chapter 4. In this light, it is reasonable for the user to set the initial tap water temperature $T_{\mathrm{d}}(0)$ equal to $T_{\mathrm{c} \text { max }}$ so that the thermal discomfort is minimal (no solid region in case (a) in Figure 5.5). keep the WH switched off Alternatively, the user thermal discomfort can be provided at the zero level by providing the tap water temperature at the Lower User Comfort Boundary $T_{\mathrm{d}}(t)=T_{\text {c.min }}(t)$ (in our example $T_{\text {c.min }}(t)=T_{\text {c.min }}=$ const ), for example, by means of the flow control introduced in Chapter 4.

Since the demanded thermal energy during the considered WA is nothing but $E_{\mathrm{d}}=$ $C_{p} \dot{m}_{\mathrm{d}} \int_{t=0}^{10 \min } T_{\mathrm{d}}(t) d t, \dot{m}_{\mathrm{d}}=$ const, we can estimate the thermal energy delivered to the user

\footnotetext{
${ }^{1}$ The tap water temperatures above the Upper Comfort Boundary represent a "no-go" zone, because of the risk of skin scalding.
} 
in each of the cases by calculating the areas below the tap water temperature graphs $T_{\mathrm{d}(t)}$ in Figure 5.5. The hatched regions highlighting the areas below the curves $T_{\mathrm{d}}(t)$ in Figure 5.5 graphically represent $E_{\mathrm{d}}(t)$ in case of (a), (b), (c) and the flow control.

Although both the case (a) and the flow control bring zero thermal discomfort to the user, the demanded thermal energy $E_{\mathrm{d}}(t)$ provided during the WA in each case are not equal.

It can be seen in Figure 5.5 that the demanded energy $E_{\mathrm{d}}(t)$ is lower in case of the flow rate control $\left(T_{\mathrm{d}}(t)=T_{\mathrm{c} \text { min }}\right)$ in comparison with the case (a) although they both result in the zero thermal discomfort. This holds for any curve fitting the comfort zone. However, if the tap water temperature curve $T_{\mathrm{d}(t)}$ drops below the Lower Comfort Boundary (as in the cases (b) and (c)), the user will experience the thermal discomfort, even though the demanded energy might be lower than that in the flow control. In our example, the tap hot water provided by the flow control is better than case (a) in terms of the demanded energy $E_{\mathrm{d} \text { flow ctrl. }}(t)<E_{\mathrm{d} \text { case(a) }}(t), t \in\left[0, \Delta t_{\mathrm{WA}}\right]$ (consequently, the electricity consumption

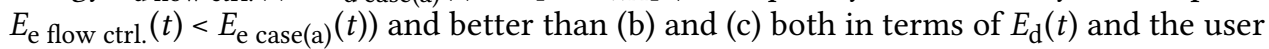
thermal comfort.

Let us show in more detail how the decrease of the demanded energy $E_{\mathrm{d}}(t)$ in case of the flow rate control can influence the electricity consumption of the WH. Consider three cases of hot water consumption and related tank water temperature recovery periods depicted in Figure 5.6(a). The cases \#1 and \#2 correspond to the situation where the mixing proportion of hot and cold water $k$ is set manually by the user at the beginning of the WA, while the case \#3 represents the flow control. All three cases are carried out under the same initial tank water temperature $T(0)=65^{\circ} \mathrm{C}$ and different cold and hot water flow rates. The energy balances in each case are represented in the form of the pie charts for the moment $t=\Delta t_{\mathrm{WA}}+\Delta t_{\mathrm{rec}, i}, i \in[1,3]$ after the tank water temperature recovery to $70^{\circ} \mathrm{C}$ in Figure 5.6(b). The green color of sectors indicates the energy input into the system taken with a positive sign, the pink color signifies the energy output with a negative sign, while the blue color shows the change of the internal energy in the system and is taken with a negative sign, so that by summing up all the values one can obtain zero.

It follows from Figure 5.6(a) that the tank water temperature at the end of the WA is higher in case of the flow rate control (\#3), as compared to the cases \#1 and \#2 $T_{3}\left(t_{\mathrm{WA}}\right)>$ $T_{2}\left(t_{\mathrm{WA}}\right)>T_{1}\left(t_{\mathrm{WA}}\right)$. This is can be explained by the reduced demanded energy in the case \#3 as shown in Figure 5.6(b). Therefore, the recovery time $\Delta t_{\text {rec3 }}$ in the case \#3 is the shortest, and the electricity consumption $E_{\mathrm{e}}\left(t_{\mathrm{WA}}+\Delta t_{\mathrm{rec}, 3}\right)$ is the smallest.

However, in some situations, the flow control can result in a greater electricity consumption, as for example, in case of long WAs. For instance, if the considered WA takes 20 [min], the demanded energy in the case \#3 will be greater than that in the cases \#1 and $\# 2$, hence the inequality $\Delta t_{\text {rec } 3}>\Delta t_{\text {rec1 }}>\Delta t_{\text {rec2 }}$ holds and the electricity consumption $E_{\mathrm{e}}\left(t_{\mathrm{WA}}+\Delta t_{\mathrm{rec}, 3}\right)$ is the highest as shown in Figure 5.8.

In the above examples, the tank water temperature is governed by the deadband control, while the initial tank water temperature $T(0)$ remains the same in all the cases. Let us now consider what happens in case of the pre-heating control of the $\mathrm{WH}$. As before, we compare three cases where the proportion of hot and cold water $k$ is set manually (the cases \#1 and \#2) and where it is set automatically by the flow control (the case \#3). By contrast to the previous examples, the tank water is heated now in compliance with the 


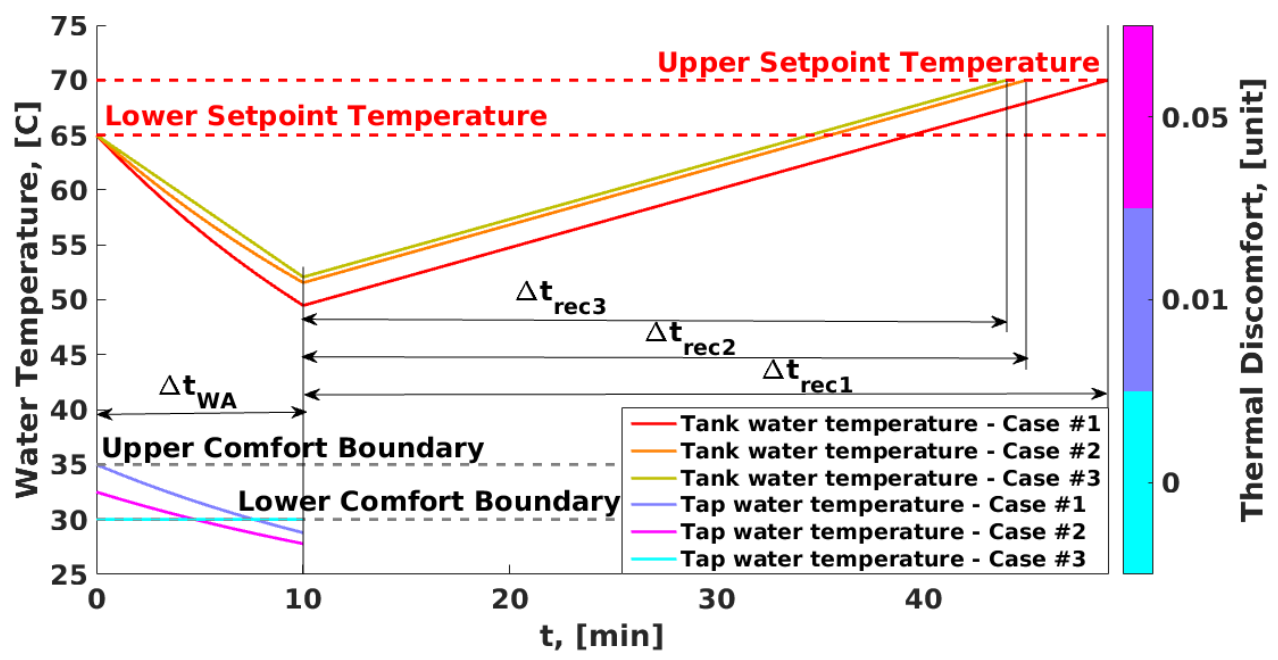

(a)
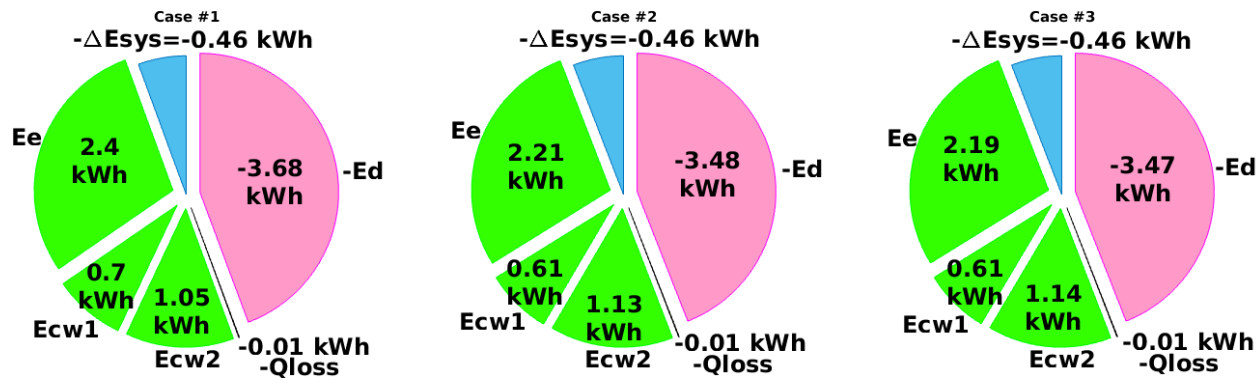

(b)

Figure 5.6: Positive example - Electricity savings during the flow control (a) temperature graphs, (b) energy balances.

pre-heating control, i.e. the tank water temperature at the beginning of the WA $T\left(t_{\mathrm{WA}}\right.$ str. $)$ is set according to the user thermal comfort during the WA. The effect of the reduced demanded energy $E_{\mathrm{d}}(t)$ during the pre-heating control is illustrated in Figure 5.8.

As one can find in Figure 5.8, there is no need to have the tank water temperature equal to $65^{\circ} \mathrm{C}$ at $t_{\mathrm{WA} \text { str. }}=120$ [min] (as in the previous examples) in order to provide the zero thermal discomfort in the case \#3. Although both the cases \#1 and \#3 are fully comfortable for the user, the tank water temperature at the beginning of the WA $T\left(t_{\mathrm{WA}}\right.$ str. $)$ is significantly higher in the case \#1 due to the higher energy demand $E_{\mathrm{d}}(t)$. As a result, the preheating time $\Delta t_{\text {pre3 }}$ is the shortest and the electricity consumption is the smallest in the case \#3 as compared against the cases \#1 and \#2.

To summarize the above considerations, two conditions must be fulfilled to enable the electricity savings by means of the flow control: 


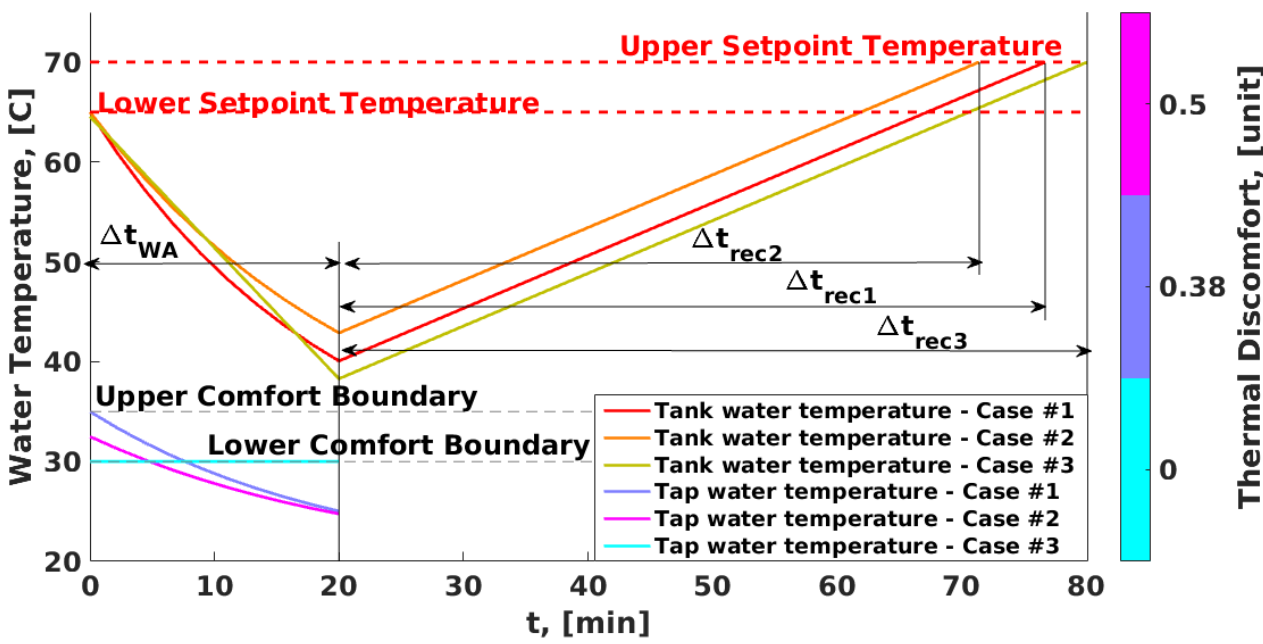

(a)
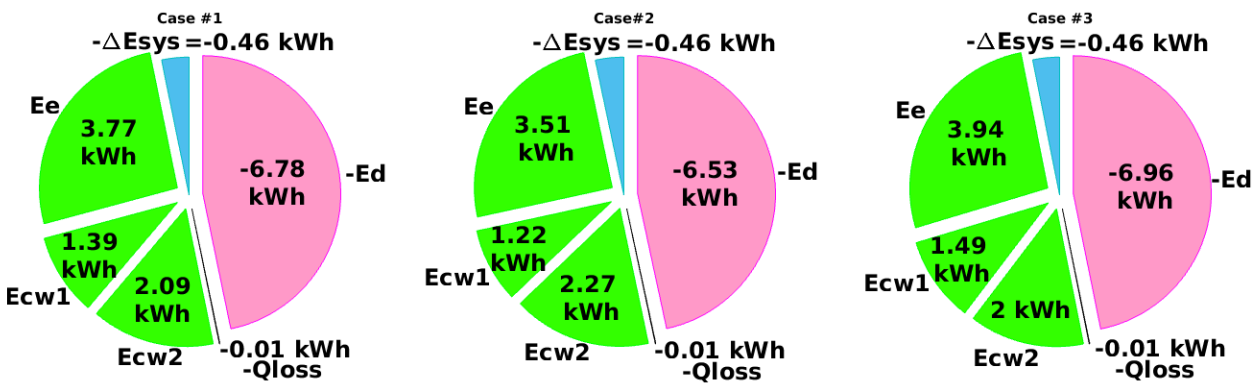

(b)

Figure 5.7: Negative example - Excessive electricity consumption during the flow control (a) temperature graphs, (b) energy balances.

- the user should be able to tolerate the tap water temperature drops during WAs ${ }^{2}$;

- the area below the temperature curve $T_{\mathrm{d}}(t)$ should be smaller than that in case of the fixed mixing proportion $k$ in the tap during the WA.

Noteworthy, there exist modern tapware solutions that like the flow control allow for reduction of $E_{\mathrm{d}}(t)$. Examples of such solutions include but not limited to taps with flow limiting device, low flow shower heads, thermostatic mixing taps (TMVs) and taps with cold water supply in middle position. However, such devices are (a) manually controlled by the user; (b) hard to model due to complex physical principles and(or) unknown physical properties of the materials used in them (e.g., TMVs); (c) used as independent solutions, i.e. their operation is not accounted for in the WH control.

${ }^{2}$ Not a stopping factor because the tap water temperature usually drops during the deadband control. 


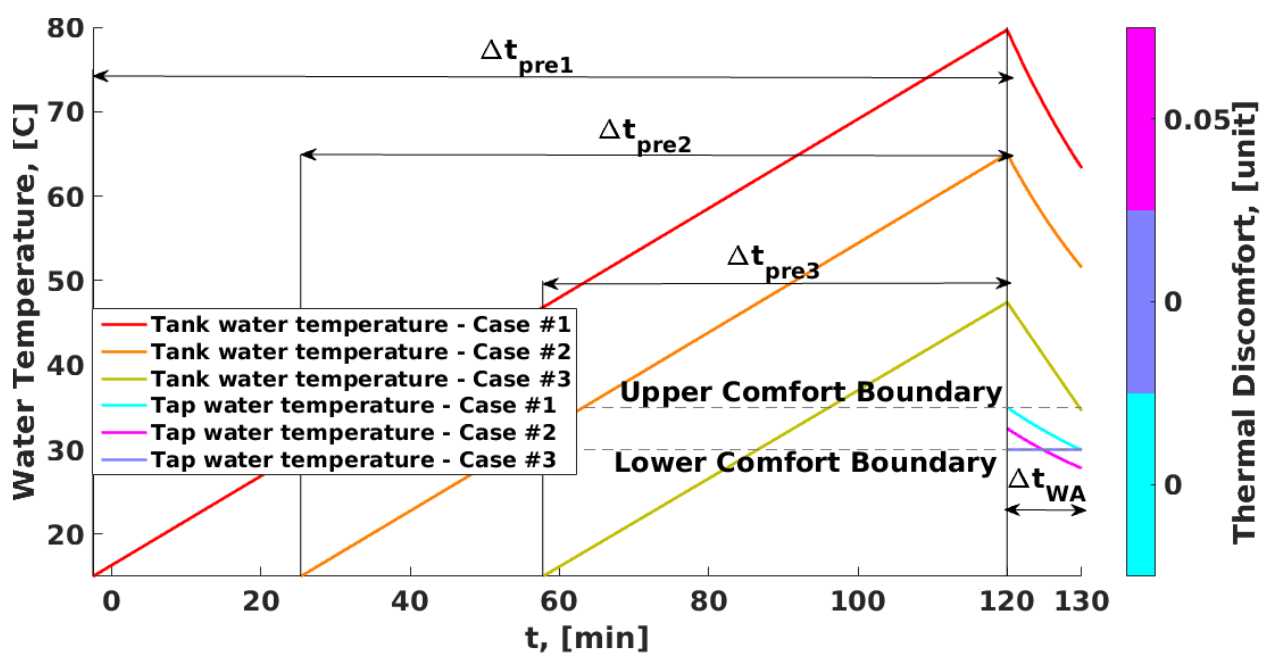

(a)
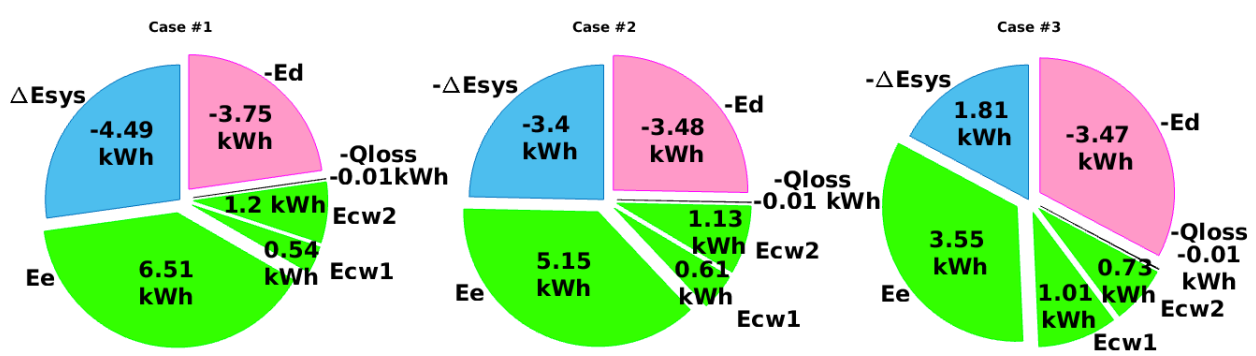

(b)

Figure 5.8: Effect of the pre-heating control.

\section{Other Specifics of OUr Approach}

Aside from the reduction of the heat loses during the stand-by periods and the alignment of the tap water temperature to the lowest edge of the user comfort zone $T_{\mathrm{d}}(t) \rightarrow T_{\text {c.min }}(t)$ noted in Section 5.4.2, other distinguishing features are briefly highlighted below.

The specifics of our approach as opposed to other approaches for WH scheduling is that it is activity-based. That is to say, the approach scales down the hot water consumption to the level of water activities (WAs) in order to accurately account for the user thermal comfort requirements and the user thermal tolerance.

The approach appeals to user comfort preferences associated with individual WAs. The user comfort requirements are the main driver of electricity consumption in this respect, i.e. the heat is built up in the WH in conformity with the desired user comfort.

In contrast to the existing approaches that treat user comfort only as a difference between instantaneous values of the water temperature and some predefined threshold(s) $[52,64]$, we incorporate the user thermal comfort model as in Equation 3.5 in Chapter 
3. The comfort model allows to account for both environmental variables, i.e. not related to the user (e.g., the tap water temperature), and also personal comfort variables (e.g., the skin sensitivity).

Another distinguishing feature of the approach is in the absence of the thermostat setpoints. In other words, the maximum tank water temperature is only restricted by the safety allowed threshold $\left(T_{\text {wh } \max }=90^{\circ} \mathrm{C}\right)$, rather than by the upper setpoint temperature as in the deadband control (usually $65-70^{\circ} \mathrm{C}$ ). By letting the tank water temperature be above the usual $70^{\circ} \mathrm{C}$, we can satisfy the user thermal comfort even during the intense WAs. On the other hand, by setting the temperature below the usual lower thermostat setpoint $55-60^{\circ} \mathrm{C}$, we can reduce the heat losses.

With respect to the adopted pre-heating mechanism, the problem with hazardous contamination of the tank by bacteria Legionella is solved by introducing sanitary cycles. They can be run at night time to kill dangerous bacteria, if the tank water temperature did not reach the sanitary value the previous day.

\subsubsection{Assumptions}

Given the hot water system setup presented in Figure 4.3 (Chapter 4), we make the following assumptions in our approach to the energy-comfort problem:

1. all the technical parameters of the WH are known;

2. multiple WAs occur on a timescale of a day;

3. WAs may overlap in time in case multiple tapping points are used in parallel;

4. all the time-related and comfort-related parameters of WAs are available from an ideal forecast;

5. the end-user sets the comfortable tap water flow rate $\dot{m}_{\mathrm{d}}(t)$ at the beginning of each WA;

6. the proportion $k=\frac{\dot{m}}{\dot{m}_{\mathrm{cw}}}$ between the hot water flow rate $\dot{m}$ and the cold water flow rate $\dot{m}_{\mathrm{cw}}$ is automatically set by the controller of the mixer tap. Considering scenarios of the hot water usage with and without user requirement for a stable tap water temperature, we distinguish between two options for the tap water provision:

(a) $T_{\mathrm{d}}(t)=v a r$ where $k$ is set only at the beginning of the WA and further remains unchanged;

(b) $T_{\mathrm{d}}(t) \approx T_{\text {c.min }}(t)$ where $k$ is adjusted by the flow control during the WA.

The user may override both types of control at any point of time;

7. the tank water temperature $T_{\text {wh, } \text { max }}$ should never exceed $T_{\text {wh } \max }=90^{\circ} \mathrm{C}$;

8. the cold water temperature $T_{\mathrm{cw}}=15^{\circ} \mathrm{C}=$ const and the ambient temperature $T_{\mathrm{amb}}=$ $24^{\circ} \mathrm{C}=$ const during a day;

9. the initial tank water temperature $T(0)$ is given. 


\subsubsection{Proposed Energy Model}

This section introduces a new control mechanism for hot water providence at home. In the heart of the proposed control mechanism, there is an "energy model" responsible for finding the best schedule of the WH heating periods and the hot water flow rates on a discrete planning horizon of 24 hours.

The input to the discrete-time energy optimization model is a profile of hot water activities together with associated user thermal comfort preferences. Given $N_{\mathrm{WA}}$ - number of WAs with the indices $k \in\left[1, N_{\mathrm{wA}}\right]$ the input consists of:

- the start times $t_{\mathrm{WA}, k}$ and durations $\Delta t_{\mathrm{WA}, k}$ of WAs;

- the tap water flow rates $\dot{m}_{\mathrm{d}, k}$;

- the user thermal comfort zones defined by the lower and upper comfort boundaries $\left[T_{\text {c.min }, k}, T_{\text {c.max }, k}\right]$;

- the user thermal comfort tolerances $\Delta_{\text {tol }, k}^{+}$and $\Delta_{\text {tol }, k}^{-}$;

- the initial tank water temperature $T(0)$ at the beginning of the planning horizon.

Based on the above input and assumptions in Section 5.4.3, the energy model seeks the best time intervals to switch the WH on/off on the discrete timescale and the best tank water temperature values that minimize the daily electricity consumption while ensuring the required user thermal comfort. This is achieved by:

- minimization of the heat losses;

- the tap water temperature provision at the lowest, yet acceptable to the user, value (Assumption 6);

Firstly, the energy model deals with optimization of the heat injections into the $\mathrm{WH}$ and decides upon the state of the $W H$, i.e. switched on or off, on every time interval of the discretized timescale. Secondly, the model finds the best tank water temperature values. These temperature values are, on the one hand, associated with the best states of the heating elements and, on the other hand, incorporate the information the best hot water flow rates. In other words, the energy model does not explicitly return the values of the best hot water flow rates, but rather produces the tank water temperature values that link the best states of the heating elements to the hot water flow rates found implicitly by the model. Two types of variables, the state of the WH heating elements and the tank water temperature, are the decision variables of the energy model.

The output of the energy model are two vectors $\mathbf{x}=\left\{x_{i} \mid x_{i} \in\{0,1\}, i \in[1, N]\right\}_{1 \mathrm{x} N}$ and $\mathrm{T}=\left\{T_{i} \mid T_{i} \in\left[T_{\mathrm{cw}}, T_{\text {wh max }}\right], i \in[1, N]\right\}_{1 \mathrm{x} N}$, where $N$ is the number of time intervals on the discrete timescale. The binary vector $\mathbf{x}$ stands for the states of the $\mathrm{WH}$ heating elements (on/off) to be set during a day. The vector $\mathrm{T}$ specifies the tank water temperature values to be established at the end of the $i$-th time interval of the discrete timescale. Based on the resulting values in $\mathbf{x}$ and $\mathrm{T}$ it is possible to restore the corresponding values of the hot water flow rates $\dot{\mathbf{m}}=\left\{\dot{m}_{i} \mid \dot{m}_{i} \in\left[0, \dot{m}_{\mathrm{d}, i}\right], i \in[1, N]\right\}_{1 \mathrm{x} N}$ by using Equation 2.2. 


\subsubsection{Proposed Control Mechanism}

This section describes the building blocks of the proposed control system and how they communicate with each other. After that we introduce the energy model that is the "brains" of the system.

The proposed system rests on two types of components. Each component type operates in a different physical location and carries out a different task. The system consists of a single WH Node and (possibly multiple) TapNode(s) that can communicate with each other as shown in Figure 5.9. The WH Node is located on the side of the WH, whereas the TapNodes are mounted at the hot water mixer taps in a house. Both the WH Node and TapNode(s) deal with two (time spaced) tasks of finding the best control schedules (or plans) and implementing them in practice. The system works as follows.

Given a profile of WAs and comfort preferences for a day ahead, the system first solves either the water pre-heating problem in Equation 4.4 or the flow control problem in Equation 4.15 independently for each of the WAs with respect to two scenarios of the hot water usage $T_{\mathrm{d}}(t)=v a r$ and $T_{\mathrm{d}}(t) \approx T_{\text {c.min }}(t)$ in Assumption 6. More specifically, we break a continuous scale of tank water temperatures [ $\left.T_{\mathrm{cw}}, T_{\mathrm{wh}, \max }\right]$ into $M$ even zones and solve the pre-heating (or the flow control) problem for every possible value $T_{j, k} \in$ $\left[T_{\mathrm{cw}}, T_{\mathrm{wh}, \text { max }}\right], j \in[1, M]$ that the tank water temperature can take at the beginning of the $k$-th WA. The result of this step is a matrix of the hot water flow estimates $\widetilde{\dot{\mathbf{m}}}=\left\{\widetilde{\dot{m}}_{i, j} \mid \widetilde{\dot{m}}_{i} \in\right.$ $\left.\left[0, \dot{m}_{\mathrm{d}, i}\right], i \in[1, N], j \in[1, M]\right\}_{N \mathrm{x} M}$. The matrix $\widetilde{\dot{\mathrm{m}}}$ allows to find the tank water temperature value $T_{i+1}$ at the end of any $i$-th interval $i \in[1, N]$ given the temperature value at the beginning of the interval $T_{i}$. This matrix is further utilized as input to the energy model. Therefore, the solutions of the energy model account for the best hot water flow rates of the pre-heating control (or flow control) for individual WAs. A more detailed discussion of input to the energy model is given in Section 5.4.8.

Next, the WH Node executes the energy model and finds the water heating schedule $\mathbf{x}$

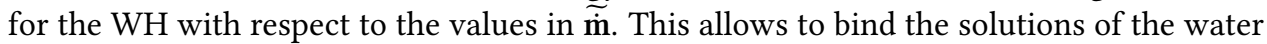
heating control with control of the flow rates in the TapNode(s). Furthermore, the energy model returns the vector of the tank water temperatures $\mathbf{T}$. The vectors $\mathbf{x}$ and $\mathbf{T}$ allow for finding the corresponding hot water flow rates $\dot{\mathbf{m}}$. The WH Node implements the solutions in $\mathbf{x}$ by switching the WH heating elements on and off during the day while sending the tank water temperature readings and the derived vector $\dot{m}$ to the TapNode(s).

The TapNodes receive the actual tank water temperature readings $\mathrm{T}$ from the $\mathrm{WH}$ Node online and based on the forecasted values of the tap water flow rates $\dot{m}_{\mathrm{d}, i}, i \in[1, N]$ and the received values in m maintains the needed proportion between the cold and hot water flow rates in the mixer tap with regards to two possible scenarios of the hot water usage in Assumption 6.

In general, WHs allow for two types of control actions to implement the best water heating schedule, namely (1) the switching of their power supply on/off and (2) the regulation of their thermostat setpoints. Both of these actions influence the WH heating elements by turning them on or off. Since different WH models may have different technical realization, we consider only option (1) in our approach, although the energy model responsible for finding the heating schedule also yields the best tank water temperature settings that could be maintained by means of the thermostat regulation (2).

So far, we have discussed the technical implementation of the control system. Let us 


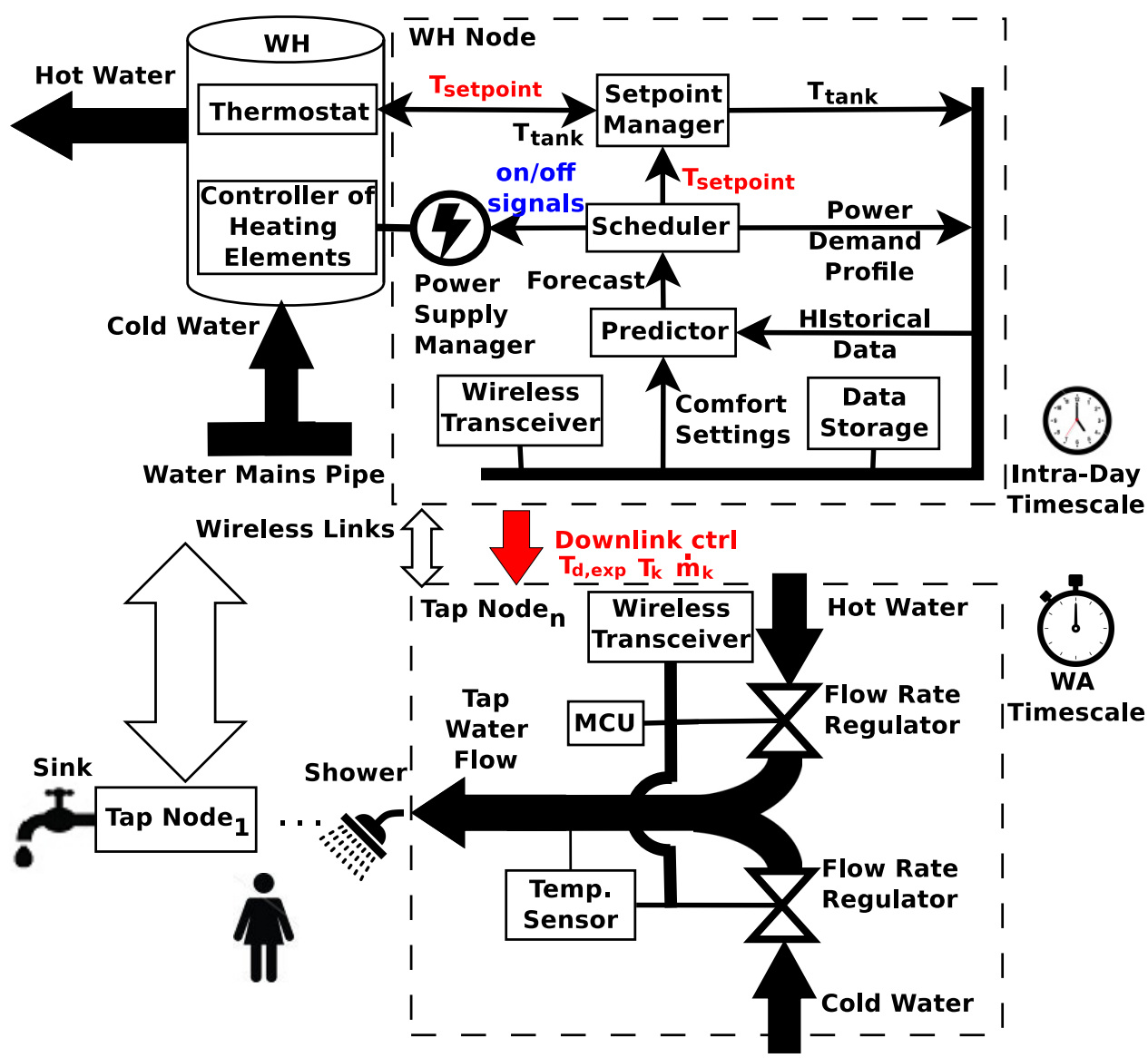

Figure 5.9: Logical blocks of control mechanism.

now elaborate on the gears that allow for finding the water heating schedule and hot water flow rates to solve the energy-comfort problem formulated in Section 5.4.1.

Firstly, the operation of the WH Node and TapNode(s) is based upon a temporal decomposition of their control programs into two discrete timescales, namely the intra-day timescale and the WA timescale. The energy model executed by the WH Node employs the intra-day timescale with a planning horizon of 24 hours. The solutions of the energy model are thus the discrete vectors $\mathbf{x}$ and $\dot{\mathbf{m}}$ of the same size as the intra-day timescale.

As opposed, the flow control of a single TapNode is implemented on the WA timescale, i.e. on the level of a single WA. Which means that the control horizon of the TapNode is limited to the WA duration. Taking into account multiple WAs during a day, there are multiple WA timescales each with its own planning horizon. The solutions of the flow control for any WA is a set of vectors $\left\{\dot{\mathbf{m}}, \dot{\mathbf{m}}_{\mathrm{cw}}\right\}$ of the size equal to the size of the corresponding WA timescale. 


\subsubsection{INTRA-DAY TIMESCALE}

As mentioned in the previous section, the WH Node operates on the discrete intra-day timescale.

Let $N$ be the total number of time intervals on the evenly discretized timescale of a day, the intra-day timescale. Also, let the symbol $i \in \mathbb{Z}_{+}, 1 \leq i \leq N$ denote the $i$-th interval that takes the time $\Delta t[\mathrm{~min}]$. Then, the real time $t$ [sec] elapsed from the beginning of the day points to the interval with index $\left\lfloor\frac{t}{60 \Delta t}\right\rfloor+1^{3}$. Conversely, the time elapsed from the beginning of the day till the start of the $i$-th interval can be represented as 60(i-1) $\Delta t$ [sec]. There are $N \triangleq\left[\frac{24 * 60}{\Delta t}\right\rfloor \geq 1$ [min] intervals within the time horizon of 24 hours.

The thermal discomfort experienced by the user during the $i$-th interval while performing the $k$-th WA $k \in\left[0, N_{\mathrm{wA}}\right]$ can be re-written as:

$$
D_{\mathrm{T}, i}=F_{\mathrm{T}, k}\left(T_{\mathrm{d}, i}\right) A_{\mathrm{T}, i},
$$

where $F_{\mathrm{T}, k}$ is the user thermal ${ }^{4}$ tolerance function for the WA $k ; T_{\mathrm{d}, i}$ stands for the tap water temperature $T_{\mathrm{d}}(t)$ realization ${ }^{5}$ during the $i$-th interval of time; $A_{\mathrm{T}, i}$ is the area ${ }^{6}$ between $T_{\mathrm{d}, i}$ and the Lower Comfort Boundary $T_{\mathrm{c} . \mathrm{min}, i}$ of the user thermal comfort zone for the $k$-th WA.

If the duration of the $k$-th WA $\Delta t_{\mathrm{wA}, k}>\Delta t$, the total user thermal discomfort during the $k$-th WA can be considered as a sum of the thermal discomfort values on the adjacent time intervals $\{i-1, i, i+1, \ldots, i+n\}$ occupied by the $k$-th WA, i.e. if the $k$-th WA overlaps $n$-number of intervals, then the thermal discomfort becomes $D_{\mathrm{T}, k}=\sum_{i=1}^{n} D_{\mathrm{T}, i}$.

Similarly, the total thermal discomfort caused to the user(s) from the beginning of the day till the start of the $i$-th interval can be expressed as $D_{\mathrm{T}}(t)=\sum_{p=0}^{i} D_{\mathrm{T}, p}, 1 \leq i \leq N$.

\subsubsection{WA TIMESCALE}

The TapNode operates on the WA timescale restricted by the start time and the end time of a certain WA that it serves.

The place of the WA timescale and its relation to the intra-day timescale is illustrated in Figure 5.10 .

\footnotetext{
${ }^{3}$ The operator [.] is designated for the "floor" function.

${ }^{4}$ The symbol "T" stands for the user "thermal" comfort, unlike the tap water flow related comfort.

${ }^{5}$ Although $T_{\mathrm{d}}(t)$ is a continuous function of time during the WA, we index it to show that we talk about its certain values restricted by the domain $t \in[60(i-1) \Delta t, 60 i \Delta t][\mathrm{sec}]$.

${ }^{6} \mathrm{In}$ fact, $A_{\mathrm{T}, i}$ is a continuous function of time within the $i$-th interval, i.e. it is computed as a difference of integrals of $T_{\text {c.min }}=$ const and $T_{\mathrm{d}}(t)$ over $t$ (recap, $T_{\text {c.min }} \leq T_{\mathrm{d}}(t) \leq T_{\text {c.max }} A_{\mathrm{T}, i}=0$, while $T_{\mathrm{d}}(t)>T_{\text {c.max }}$ is a "no-go" zone $\left.A_{\mathrm{T}, i}=\infty\right)$, we index it to refer to the values of $T_{\mathrm{c} \text {.min }}$ and $T_{\mathrm{d}}(t)$ in the domain $t \in[60 i \Delta t, 60(i+1) \Delta t]$ [sec] .
} 


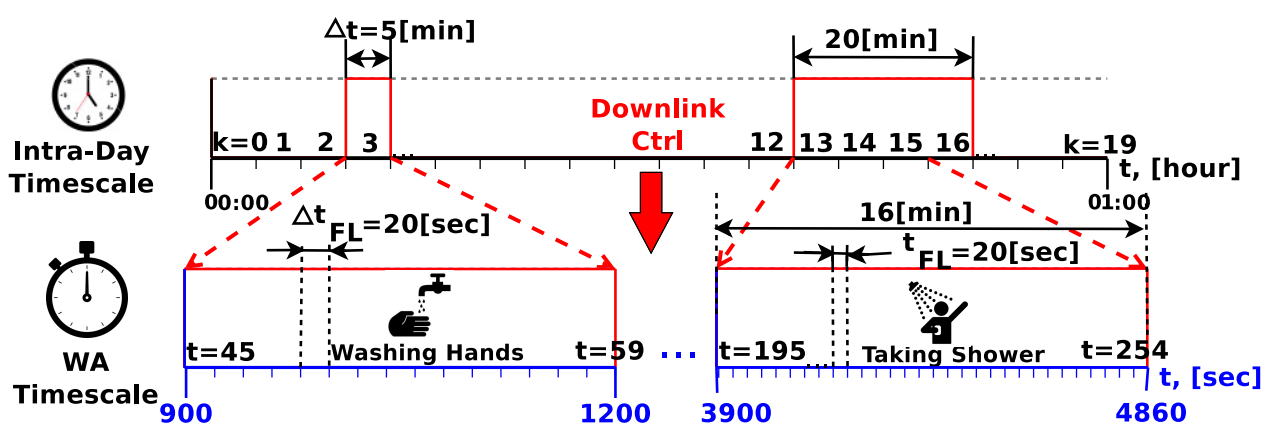

Figure 5.10: Intra-day and WA timescales.

As it can be seen from Figure 5.10, there may exist multiple WA timescales during a day each suiting an individual WA. For the purpose of simplicity, we unite all the individual WA timescales into a single WA timescale by the end-to-end indexing of their intervals during a day. As Figure 5.10 shows there might be gaps in between of the WAs, thus we can speak of activation of the WA timescale at the start of the WAs and deactivation at their end times.

Let $N_{\mathrm{FL}}$ be the number of intervals of the evenly discretized WA timescale active during a day. Also, let the symbol $w \in \mathbb{Z}_{+}, 1 \leq w \leq N_{\mathrm{FL}}$ denote the index of the $w$-th interval with the size $\Delta t_{\mathrm{FL}}[\mathrm{s}], 0<\Delta t_{\mathrm{FL}} \leq \Delta t$ on the WA timescale. Then, the number of intervals active during a day is $N_{\mathrm{FL}} \triangleq\left\lfloor\frac{24 * 60 * 60}{\Delta t_{\mathrm{FL}}}\right\rfloor$, and the maximum number of active intervals inside one interval of the intra-day timescale is $N_{\mathrm{SF}} \triangleq\left\lfloor\frac{\Delta t}{\Delta t_{\mathrm{FL}}}\right\rfloor \geq 1$.

The WA timescale and the intra-day timescale are interrelated as follows. If $\kappa_{\mathrm{SF}}(w) \triangleq$ $\left\lfloor\frac{w}{N_{\mathrm{SF}}}\right\rfloor$, then the index of the interval on the intra-day timescale where $w$ belongs to can be defined as $\kappa_{\mathrm{SF}}(w)=i \Leftrightarrow w \Delta t_{\mathrm{FL}} \in[i \Delta t,(i+1) \Delta t)$. And the other way around, indices of intervals on the WA timescale that are active along the $i$-th interval of the intra-day timescale, i.e. indices in the set $W=\{w, w+1, \ldots, w+m\}$ such that for every $w+q \in W, q \in$ $[0, m]$ holds $\kappa_{\mathrm{SF}}(w)=i$, are $w_{\min } \triangleq i\left\lfloor\frac{\Delta t}{\Delta t_{\mathrm{FL}}}\right\rfloor$ and $w_{\max } \triangleq(i+1)\left\lfloor\frac{\Delta t}{\Delta t_{\mathrm{FL}}}\right\rfloor-1$. Indices occupied by the $k$-th WA with start and end times $\left[t_{1}, t_{2}\right]$ [sec] on the WA timescale are defined as $\left[w_{\mathrm{str}}, w_{\text {end }}\right]=\left[\left\lfloor\frac{t_{1}}{\Delta t_{\mathrm{FL}}}\right\rfloor+1,\left\lfloor\frac{t_{2}}{\Delta t_{\mathrm{FL}}}\right\rfloor\right]$. Then, the thermal discomfort during the WA that occupies the intervals $\left[w_{\mathrm{str}}, w_{\mathrm{end}}\right]$ can be computed as follows:

$$
D_{\mathrm{T}}=\sum_{w=w_{\mathrm{str}}}^{w_{\text {end }}} D_{\mathrm{T}, w}
$$

where $D_{\mathrm{T}, w}$ is the thermal discomfort during the interval $w$.

\subsubsection{Formalization OF ENERgy MOdEL}

In this section, we formalize the energy model to solve the energy-comfort problem in Section 5.4.1. 


\section{Model InPuT}

In general, the tank water temperature function $T(t)$ is characterized by periods of hot water usage and stand-by periods, as illustrated in Figure 5.11 for the case of the conventional deadband control.

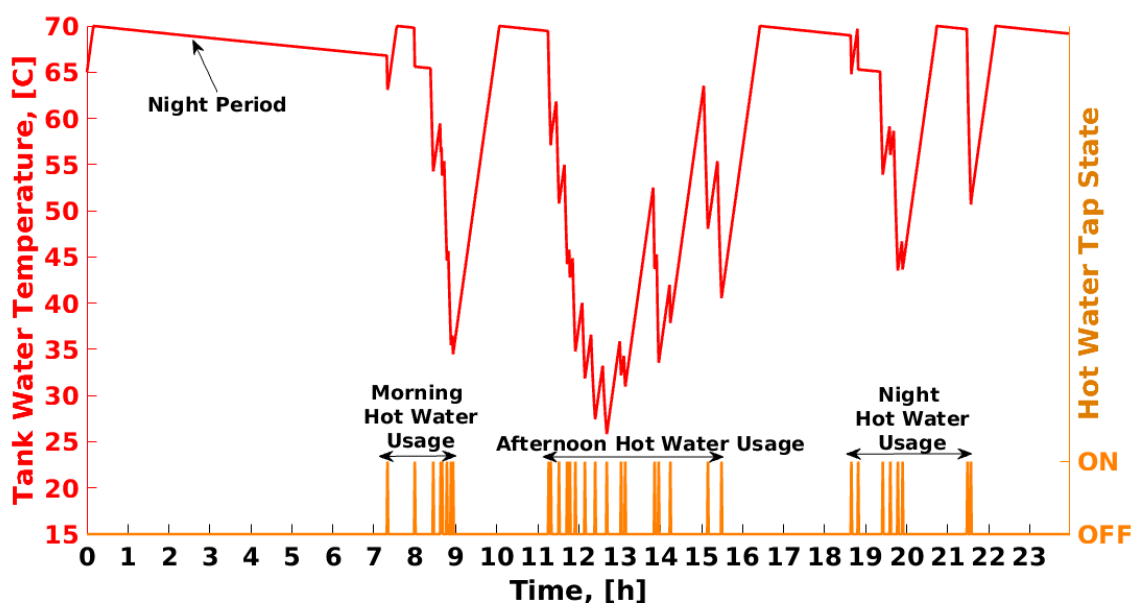

Figure 5.11: Typical hot water usage at home - Deadband control.

Although, the tank water temperature is a non-linear function in Equation 2.2, one can notice that the graph of $T(t)$ exhibits linearities for our WH model, as depicted in Figure 5.11. Therefore, any realization of the tank water temperature function $T(t)$ can be approximated by a linear piece-wise function on any (relatively small) time interval with index $i$ as follows:

$$
T_{i}=a_{i} \Delta t+T_{i-1}, T_{i} \in \mathbb{R}_{+}, i \in[1, N],
$$

where $T_{i}$ and $T_{i-1}$ are the estimates of the tank water temperature $T(t)$ at the end and the beginning of the $i$-th interval, respectively; $a_{i}$ is a tangent of the linear approximation function on the $i$-th interval.

We divide the range of allowed temperatures $\left[T_{\mathrm{cw}}, T_{\mathrm{wh}, \max }\right]$ into $M$-number of even zones $\frac{T_{\mathrm{wh}, \max }-T_{\mathrm{cw}}}{\Delta T}$ with an arbitrary size $\Delta T$ and approximate the function $T(t)$ on these intervals by applying Equation 5.3. Thus, the values of $T(t)$ that belong to the same temperature zone $j \in[1, M]$ have the same value of the tangent $a_{j}$. Considering a discrete intra-day timescale, Equation 5.3 can be then re-written for the $i$-th time interval $i \in[1, N]$ and $j$-th temperature zone $j \in[1, M]$ as follows:

$$
T_{i}=a_{j} \Delta t+T_{i-1}
$$

It is worthwhile to mention that the temperature values $T_{i}$ are real values within the temperature zones with the above breakdown of temperature scale, i.e. $T_{j-1} \leq T_{i} \leq T_{j}, T_{i} \in$ $\mathbb{R}_{+}, i \in[1, N], j \in[1, M]$.

Let $\mathbf{x}=\left\{x_{i} \mid x_{i} \in\{0,1\}, i \in[1, N]\right\}_{1 \mathrm{x} N}$ denote the states of the WH heating elements (on/off) and vector $\dot{\mathbf{m}}=\left\{\dot{m}_{i} \mid \dot{m}_{i} \in\left[0, \dot{m}_{\mathrm{d}, i}\right], i \in[1, N]\right\}_{1 \mathrm{xN}}$ define the hot water outflows from 
the $\mathrm{WH}$ on the intra-day timescale. To solve the energy-comfort problem in Section 5.4.1, we need to find such $\mathbf{x}$ and $\mathbf{m}$ that minimize the daily heat losses while satisfying the user thermal comfort needs. The state of the WH $x_{i}$ together with the value of the hot water outflow from the $\mathrm{WH} \dot{m}_{i}$ determine the tank water temperature transition process during the $i$-th time interval of the intra-day timescale.

In the following, we define the input of the energy model. At the first step, given a profile of WAs mapped onto $N$-number of intervals of the intra-day timescale, we compute all the possible tank water temperature transitions $T_{i-1, j} \rightarrow T_{i, j}, i \in[1, N], j \in[1, M]$ for each time interval $i$ and every temperature zone $j$. To solve this task, we distinguish between

- the intervals without WAs $\left(\dot{m}_{i}=0\right)$ and with WAs $\left(\dot{m}_{i}>0\right)$;

- two possible states of the WH $x_{i}=\{0,1\}$.

For the time intervals without WAs, we first compute the real (non-approximated) tank water temperature values $T_{i}, i \in[1, N]$ at the end of each $i$-th interval for each possible state of the WH $x_{i}=\{0,1\}$ by plugging the known values $T_{j}, j \in[1, M]$ at the beginning of each $j$ th temperature zone, $\dot{m}_{i}=0$ and the size of the $i$-th time interval $\Delta t$ in Equation 2.2. Second, we calculate the tangents $a_{i, j}, i \in[1, M], j \in[1, M]$ corresponding to the found temperature transitions $T_{i-1, j} \rightarrow T_{i, j}, i \in[1, N], j \in[1, M]$ by using Equation 5.4. The computed tangents are then stored in the matrices $\mathrm{A}^{+}$and $\mathrm{A}^{-}$of sizes $[N \mathrm{x} M]$ corresponding to the cases where the WH is switched on $x_{i}=1$ and switched off $x_{i}=0$, respectively. Note that at this stage the matrices contain only non-zero elements in rows whose indices correspond to the indices $i$ without WAs.

For the intervals with WAs, we solve the water pre-heating problem in Equation 4.4 or the flow control problem in Equation 4.15 independently for each of the WAs with respect to two scenarios of the hot water usage $T_{\mathrm{d}}(t)=v a r$ and $T_{\mathrm{d}}(t) \approx T_{\text {c.min }}(t)$ in Assumption 6 . More precisely, the pre-heating (or the flow control) problem is solved for each possible state of the WH $x_{i}=\{0,1\}$ provided that the temperature value $T_{i, j}, i \in[1, N], j \in[1, M]$ is equal to the temperature at the beginning of each $j$-th temperature zone. As a result, the hot water flow rates $\widetilde{\dot{m}}_{i}$ and the tank water temperature values $T_{i}$ on the intervals with WAs are found. These values are then fed into Equation 5.4 to obtain the tangents $a_{i, j}, i \in[1, M], j \in[1, M]$ of the temperature transitions. The matrices $\mathrm{A}^{+}$and $\mathrm{A}^{-}$are updated with the found values $a_{i, j}$ in the rows that correspond to the time intervals with WAs. In this way, the matrices $\mathbf{A}^{+}$and $\mathbf{A}^{-}$relate each possible input temperature value $T_{i-1, j}$ at the beginning of the $i$-th time interval to the temperature value $T_{i, j}$ at the end of the that interval via the tangent $a_{i, j}$.

At the second step, we use the obtained (non-approximated) temperature values from the first step to compute the the thermal discomfort values $D_{\mathrm{T}, i, j}, i \in[1, N], j \in[1, M]$ that the user(s) can experience during the temperature transition $T_{i-1, j} \rightarrow T_{i, j}$ on any $i$-th interval of the intra-day timescale. As before, we do the computations for two possible states of the WH $x_{i}=\{0,1\}$. Similar to the temperature transitions, the computed thermal discomfort values are arranged in two matrices $\mathrm{D}^{+}$and $\mathrm{D}^{-}$of sizes $[N \mathrm{x} M]$.

Four constructed matrices $\mathrm{A}^{+}, \mathrm{A}^{-}$and $\mathrm{D}^{+}, \mathrm{D}^{-}$define the input of the energy model. At this point the preparational steps for the energy-model are finished. The next task is to find the best vectors $\mathbf{x}$ and $\dot{\mathbf{m}}$ that minimize the heat losses while providing the required user thermal comfort. 


\section{Model Constraints}

Let us, first, introduce two auxiliary binary matrices $\gamma^{+}$and $\gamma^{-}$of size $[N \mathrm{x} M]$ whose elements $\gamma_{i, j}^{+}=\{0,1\}$ and $\gamma_{i, j}^{-}=\{0,1\}$ are responsible for selection of the transitions $T_{i-1, j} \rightarrow$ $T_{i, j}, i \in[1, N], j \in[1, M]$ from the matrices $\mathrm{A}^{+}$and $\mathrm{A}^{-}$, respectively. The vectors $\boldsymbol{\gamma}_{i}^{+}, \boldsymbol{\gamma}_{i}^{-}, i \in$ $[1, N]$ represent the elements in the rows of $\boldsymbol{\gamma}^{+}$and $\boldsymbol{\gamma}^{-}$. We index the columns in the matrices $\mathrm{A}^{+}, \mathrm{A}^{-}$and $\mathrm{D}^{+}, \mathrm{D}^{-}$with $j$ so that $A_{i, j}^{+}, A_{i, j}^{-}, D_{i, j}^{+}, D_{i, j}^{-}$are the elements at the intersections of the $i$-th row and $j$-th column in these matrices.

Then, the following constraints should hold for $\gamma^{+}$and $\gamma^{-}$on the $i$-th interval $i \in[1, N]$ :

$$
\begin{gathered}
x_{i}=\sum_{j=1}^{M} \gamma_{i, j}^{+} ; \\
x_{i}=1-\sum_{j=1}^{M} \gamma_{i, j}^{-} ; \\
T_{i}=\sum_{j=1}^{M} \gamma_{i, j}^{+} A_{i, j}^{+}+\sum_{j=1}^{M} \gamma_{i, j}^{-} A_{i, j}^{-} ; \\
T_{i} \geq \Delta T \sum_{j=1}^{M}(j-1)\left(\gamma_{i, j}^{+}+\gamma_{i, j}^{-}\right) ; \\
T_{i}<\Delta T \sum_{j=1}^{M} j\left(\gamma_{i, j}^{+}+\gamma_{i, j}^{-}\right) ; \\
M \gamma_{i, j}^{+} D_{i, j}^{+}+\sum_{j=1}^{M} \gamma_{i, j}^{-} D_{i, j}^{-} \leq d_{i} .
\end{gathered}
$$

More specifically, by introducing Constraint 5.5, we want the state $x_{i}$ of the WH on the $i$-th interval to be equal to "1" (WH is switched on), if the temperature transition selected by the selector $\gamma_{i}^{+}$is in the matrix $\mathrm{A}^{+}$. If this is the case, we want at the same time to prevent switching the $\mathrm{WH}$ on on the same interval by means of Constraint 5.6.

For any given temperature value $T_{i-1}$ at the beginning of the $i$-th time interval, we need to obtain the temperature value $T_{i}$ at the end of that interval. For instance, if the vector $\gamma_{i}^{+}$at the current interval $i$ selects the $j$-th column in $\mathrm{A}^{+}$, the tangent stored in the cell $A_{i, j}^{+}$will determine the temperature at the end of the $i$-th interval. Constraint 5.7 is responsible for obtaining the tank water temperature values at the end of each interval $i \in[1, N]$.

To obtain the index of the column that corresponds to the choice of the temperature value $T_{i}$, we introduce Constraint 5.8 and Constraint 5.9 that map the value $T_{i} \in \mathbb{R}_{+}$onto the temperature scale with $M$ even ranges discussed in Section 5.4.8.

Based on the formulation of the energy-comfort problem in Section 5.4.1, we would like the user thermal discomfort to be not greater than some acceptable level $d_{i}$ on any $i$-th time interval. By introducing Constraint 5.10, the energy model takes care of the user thermal comfort level.

Last but not the least, we need to account for Assumption 7 by limiting the tank water temperatures $T_{i}, i \in[1, N]$ below the safety allowed temperature $T_{\mathrm{wh}, \max }$ as follows:

$$
T_{i} \leq T_{\mathrm{wh}, \max }, i \in[1, N]
$$




\section{ObJective Function}

The objective of the energy-comfort problem is minimization of the daily heat losses under the required level of the user thermal comfort.

The amount of heat losses from the WH tank during the $N$-number of intervals of the intra-day timescale can be written as $Q_{\text {loss }, N}=\sum_{i=1}^{N} U A\left(T_{i}-T_{\text {amb }}\right) \Delta t$.

According to our assumptions in Section 5.4.3, $T_{\mathrm{amb}}=$ const during a day, the granularity of the intra-day timescale is fixed during a day $\Delta t=$ const too. The insulation of the WH tank $U$ and the area $A$ of its jacket remain constant. Therefore, the daily heat losses are only determined by the tank water temperature values $T_{i}, i \in[1, N]$. Taking this into account, the objective function for the energy-comfort problem can be represented by minimization of the daily average temperature. Then, the energy optimization model can be formalized as follows:

$$
\min \left[\sum_{i=1}^{N} T_{i}\right]
$$

subject to the constraints in Equation 5.5 to Equation 5.11 in Section 5.4.8.

Equation 5.12 and Equation 5.5 to Equation 5.11 specify a Linear Programming optimization problem. We solve this problem by means of Gurobi optimization software and evaluate the results in the coming section.

The solution of the energy model is two-fold. Firstly, The solution is represented by the binary vector $\mathbf{x}=\left\{x_{i} \mid x_{i} \in\{0,1\}, i \in[1, N]\right\}_{1 \times N}$ that prescribes on/off states to the WH on the intra-day timescale. Secondly, the energy model returns a vector of the tank water temperatures $\mathbf{T}=\left\{T_{i} \mid T_{i} \in\left[T_{\text {cw }}, T_{\text {wh max }}\right], i \in[1, N]\right\}_{1 \times N}$ established at the end of $N$ intervals, if the solutions in $\mathbf{x}$ are implemented.

Since the vector $\mathrm{T}$ is found with respect to the best values of the hot water outflows from the WH (incorporates them), the needed vector of the hot water flow rates $\dot{\mathbf{m}}=$ $\left\{\dot{m}_{i} \mid \dot{m}_{i} \in\left[0, \dot{m}_{\mathrm{d}, i}\right], i \in[1, N]\right\}_{1 \mathrm{x} N}$, can be restored by plugging the found values in $\mathbf{x}$ and $\mathbf{T}$ into Equation 2.2 and solving it iteratively for $\dot{m}_{i}, i \in[1, N]$.

\subsubsection{Performance Evaluation}

In this section, we evaluate the the performance of the energy model introduced in Section 5.4.8. Based on a series of simulations, we check the capability of the model to minimize the daily heat losses and, therefore, to save the electrical energy, while providing the required user thermal comfort. The simulation results are compared to the results of the conventional deadband control. For the comparison purposes, we focused on providing the user thermal comfort on the level comparable ${ }^{7}$ to the comfort level delivered by the deadband control.

We consider two scenarios of the hot water usage, namely with and without the user requirement for a stable tap water temperature (Assumption 6). We refer to these two scenarios as the control options (i) $T_{\mathrm{d}}(t) \approx T_{\text {c.min }}(t)$, meaning that the TapNode maintains the tap water temperature at the Lower Comfort Boundary and the control option (ii)

\footnotetext{
${ }^{7}$ A small deviation present in the simulation results can be explained by discretization of the intra-day timescale (in our case the granularity $\Delta t=5[\mathrm{~min}]$ ), the errors of the approximation of the temperature curves with linear functions, the applied user acceptable discomfort levels $d_{i}, i \in[1, N]$ participating in the energy model comfort constraints (Equation 5.10) and tolerances of the Gurobi optimization solver (e.g., the optimality, duality gaps).
} 
$T_{\mathrm{d}}(t)=v a r$ where the proportion between the hot and cold water flows $k=\frac{\dot{m}}{m_{\mathrm{cw}}}$ in the mixer tap is maintained by the TapNode during the entire WA.

A set of hot water demand profiles was utilized as an ideal forecast input for the energy model. The hot water usage profiles created in Load Profile Generator (LPG) software [124] contained the information about the start and end times of hot water withdrawals and the tap water flow rates $\dot{m}_{\mathrm{d}}[\mathrm{L} / \mathrm{sec}]$ per hot water activity category. The profiles were linked to the user tolerance functions $F_{\mathrm{T}, k}$ derived based on the publicly available statistical averages [119] and thermo-physiological considerations [74].

Firstly, we focused on a scenario with multiple non-overlapping in time WAs. Specifically, we calculated the amounts of the reduced heat losses and electricity savings achieved by the control options (i) and (ii) based on simulations for a single working day. We showed how the electricity savings can change with the improvement of the $\mathrm{WH}$ insulation ( $U$-factor), while keeping all other parameters of the WH model the same. Additionally, we explored how the decrease of the user comfort tolerance can affect the electricity savings attained by (i) and (ii) based on the same profile.

We further extended our simulations to a period of 8 sequential days D1-D8 to examine the effect of daily hot water consumption patterns on the heat losses and electrical consumption of the energy model. More specifically, the initial water temperature inside the WH tank was equal to the temperature of cold water $T_{0}=T_{\mathrm{cw}}=15^{\circ} \mathrm{C}$ at the beginning of the simulation period. This situation corresponds to the case of a "cold" start of the proposed control system and represents the worst case scenario. However, the initial tank water temperature in the real life might be in the range $\left[T_{\mathrm{cw}}, T_{\mathrm{wh} \max }=[15,90]^{\circ} \mathrm{C}\right.$. For example, if the proposed control was already running prior to the period of estimation, i.e. "hot" start. To exclude the effect of the assumption $T_{0}=15^{\circ} \mathrm{C}$ on the simulation results, we calculated the reduction of the heat losses and electricity savings for a weekly period D2-D8. We further demonstrated the effect of the varied $T_{0}$ on the heat losses reduction and electricity savings based on D1.

Finally, to check the applicability of the energy model to the case of WAs that overlap in time, we considered a setup where multiple tapping points were connected by a single hot water pipe that supplied hot water from the $\mathrm{WH}$, while the cold water was supplied to the tapping points via separate cold water pipes. In our scenario, two tapping points were used in parallel to perform WAs with indentical time- and comfort-related parameters.

\section{Single Day Simulations}

The simulation results obtained for the control options (i) and (ii) for a typical working day are illustrated in Figure 5.12-Figure 5.14.

It can be seen in Figure 5.13 that control (ii) $T_{\mathrm{d}}(t)=v a r$ results in higher spikes of the tank water temperature during a day than in case of (i) $T_{\mathrm{d}}(t) \approx T_{\text {c.min }}(t)$. The electricity savings reached by (i) are therefore by $2.41[\mathrm{kWh}]$ bigger than those in case of (ii). Because the flow control in (i) exploits the existing user thermal comfort tolerance, i.e. reduces the demanded thermal energy $E_{\mathrm{d}}(t)$ down to the minimum user comfortable level, there is no need to heat the water to higher temperatures, hence the electricity savings. Furthermore, both the control options (i) and (ii) result in a slightly higher user thermal comfort as compared to the deadband control. For example, the control (i) brings by about 0.15 [unit] higher user thermal comfort than the deadband control as illustrated in Figure 5.14. 


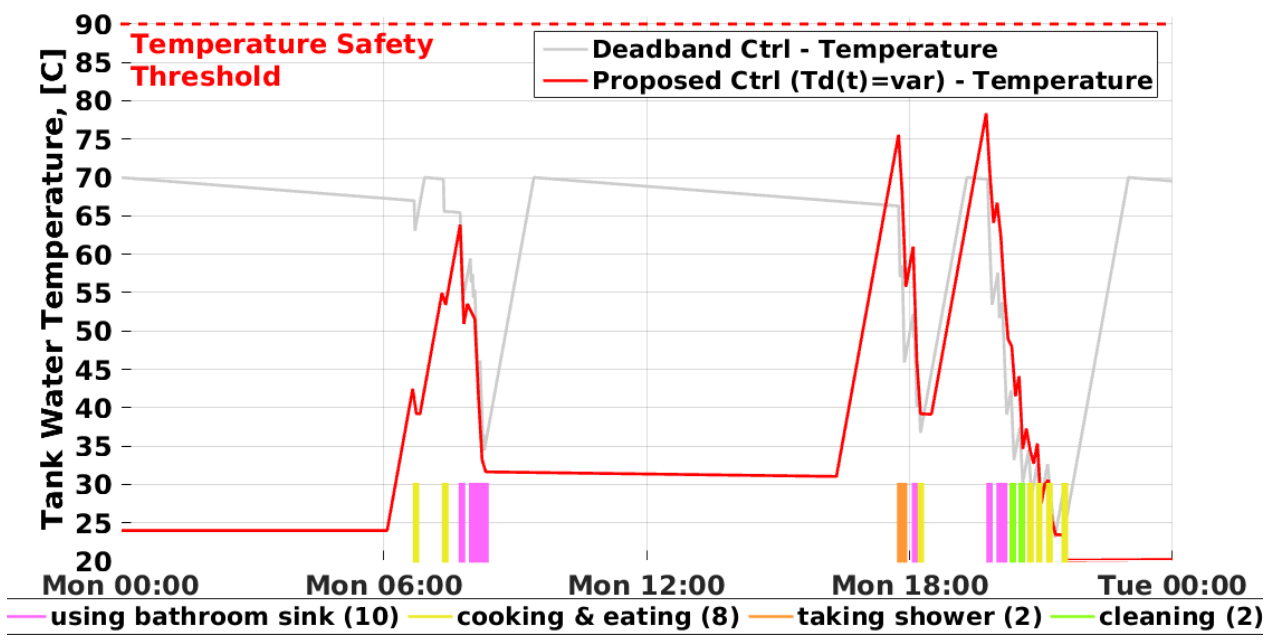

Figure 5.12: Typical working day profile (numbers in braces indicate the number of WAs).

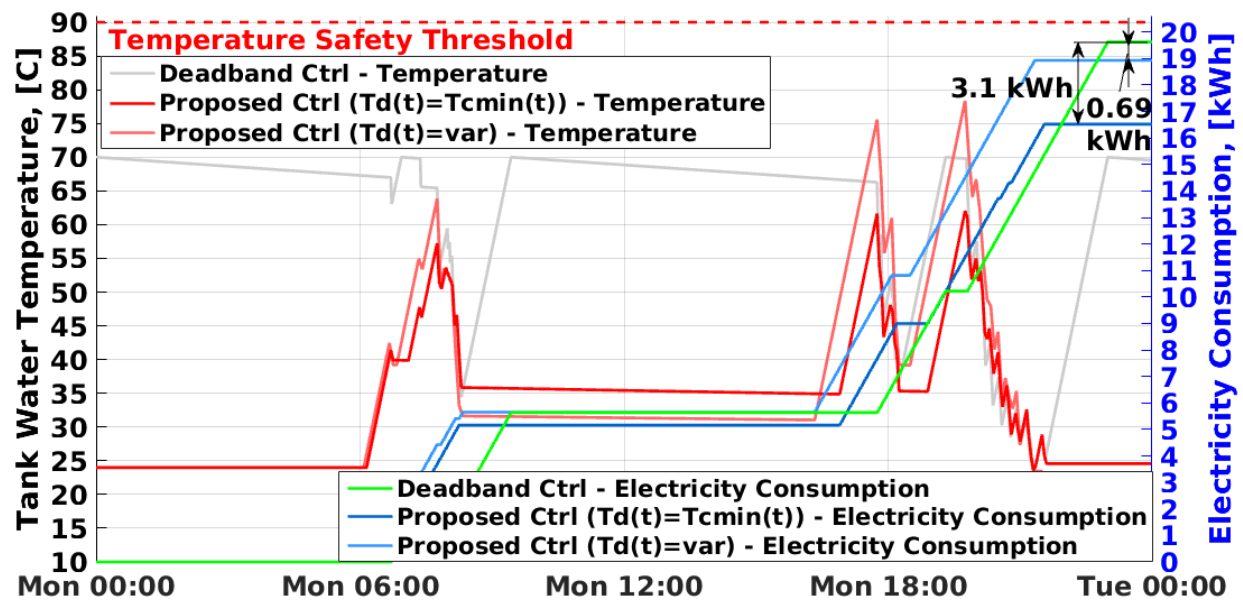

Figure 5.13: Typical working day - Electricity savings.

It is important to note that the initial tank water temperature $T(0)$ was equal to $24^{\circ} \mathrm{C}$ in case of the proposed control as against $T(0)=70^{\circ} \mathrm{C}$ in the deadband control, meaning that the initial conditions were not equal in our comparison. The amount of electricity not accounted in the comparison is equivalent to the amount of electricity that would be spent for heating the water from $T_{\mathrm{cw}}=15^{\circ} \mathrm{C}$ to $61^{\circ} \mathrm{C}$. This "hidden" portion of electricity can be used for a sanitary cycle to prevent contamination of the tank by bacteria Legionella in the proposed control. 


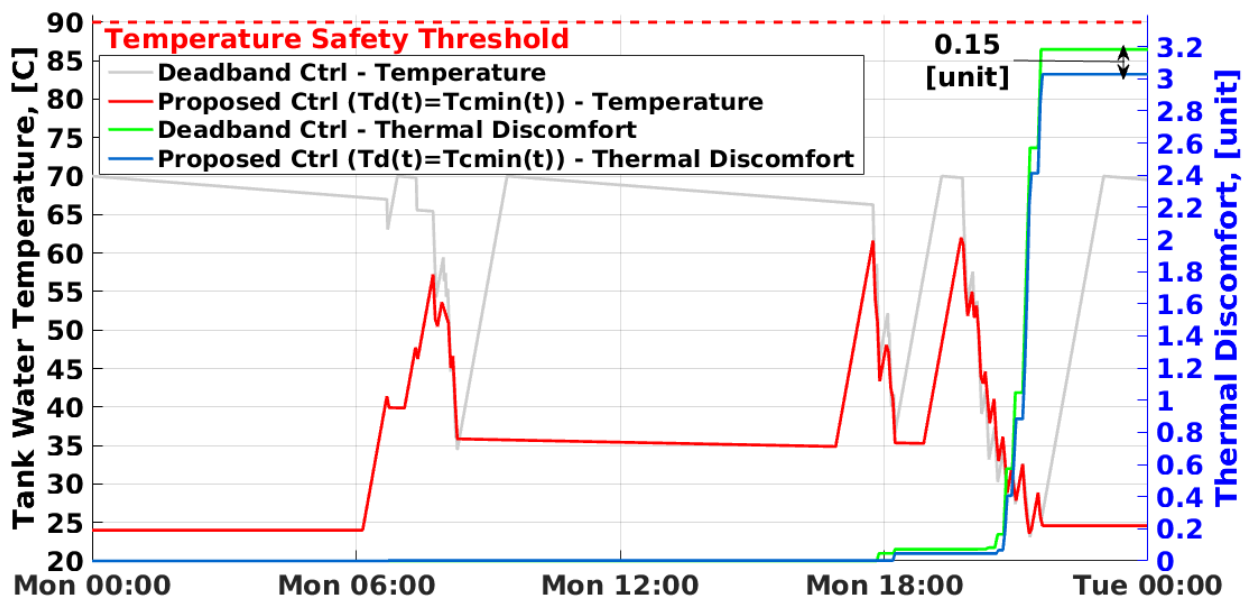

Figure 5.14: Typical working day - Thermal comfort.

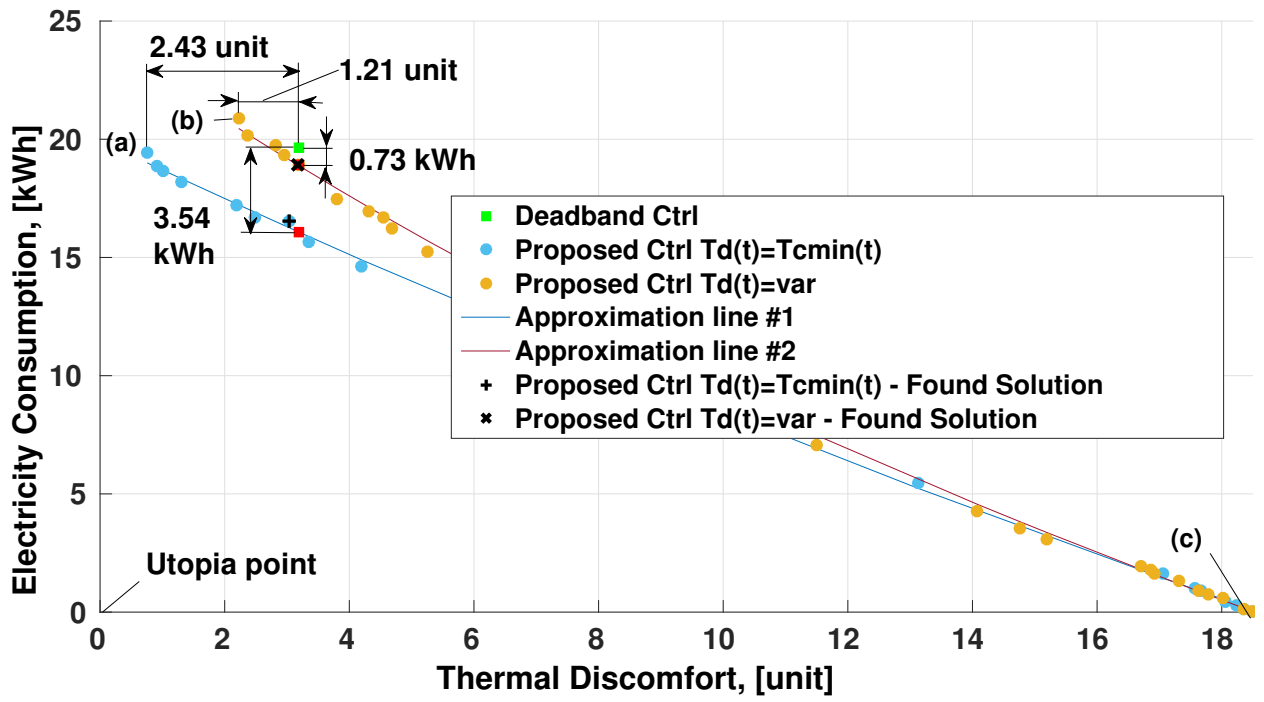

Figure 5.15: Typical working day - Pareto fronts.

In fact, the control options (i) and (ii) also allow for the user comfort values higher and lower than those provided by the deadband control. We build Pareto fronts represented by solutions of (i) and (ii) to see the maximum possible comfort levels in options (i) and (ii). The obtained Pareto fronts are depicted in Figure 5.15.

By mapping the previously found solutions for a typical working day in Figure 5.13 


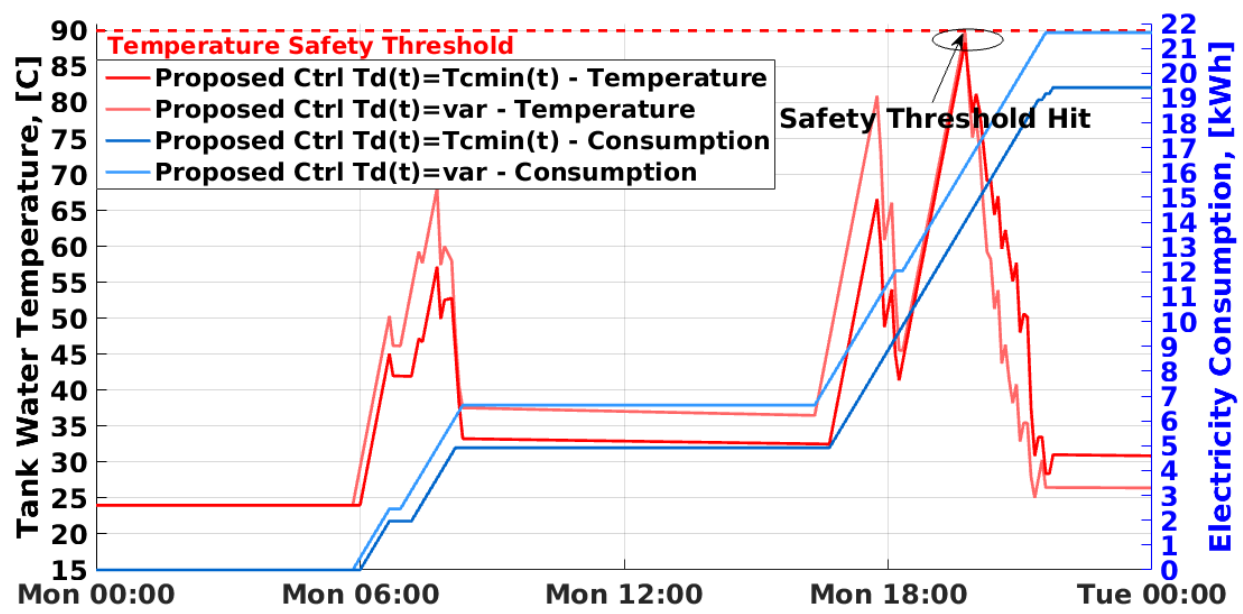

Figure 5.16: Typical working day - Electricity consumption under maximum comfort setting.

onto the Pareto fronts in Figure 5.15, one can find that the real electricity savings provided by (i) and (ii) are (i) $3.54[\mathrm{kWh}]-3.1[\mathrm{kWh}]=0.44[\mathrm{kWh}]$ and (ii) $0.73[\mathrm{kWh}]-0.69[\mathrm{kWh}]$ $=0.04[\mathrm{kWh}]$. These values are higher than those shown in Figure 5.13. This is due to a slight mismatch in comfort levels between the found solutions and the deadband control in our simulations. As it follows from Figure 5.15, the control option (i) can yield smaller user thermal discomfort under lesser electricity consumption than control (ii) in almost all the situations. The points (a) and (b) in Figure 5.15 correspond to the solutions under the maximum comfort setting in case of the options (i) and (ii), respectively. The point (c) in Figure 5.15 refers to the minimum possible thermal comfort in case of zero electricity consumption. The absolute maximum thermal comfort achieved by the control (i) is by 1.22 [unit] higher than that in case of (ii) and by 2.43 [unit] higher than in the deadband control. As can it can be seen from the Pareto fronts, it is not possible to achieve higher comfort levels than (a) and (b) in case of (i) and (ii). This is due to Constraint 5.11 on the maximum safety allowed water temperature in the tank that should not exceed $90^{\circ} \mathrm{C}$. The tank water temperature curves corresponding to solutions (a) and (b) in Figure 5.15 are illustrated in Figure 5.16 along with the achieved user thermal comfort shown Figure 5.17.

To investigate how different insulation of the WH affects energy savings of the energy model, we varied the $U$-factor of the WH model, ${ }^{8}$ while applying the same profile of a typical working day in the simulations. The resulting electricity savings attained by the control options (i) and (ii) are depicted in Figure 5.18.

First of all, Figure 5.18 shows that the electricity savings decrease for a good insulated WH in both the control options (i) and (ii). Aside from the electricity savings due to the reduced heat losses, the electricity savings in the control option (i) also arise from the

${ }^{8}$ All other technical parameters of the WH model such as the area of the WH jacket remained unchanged. 


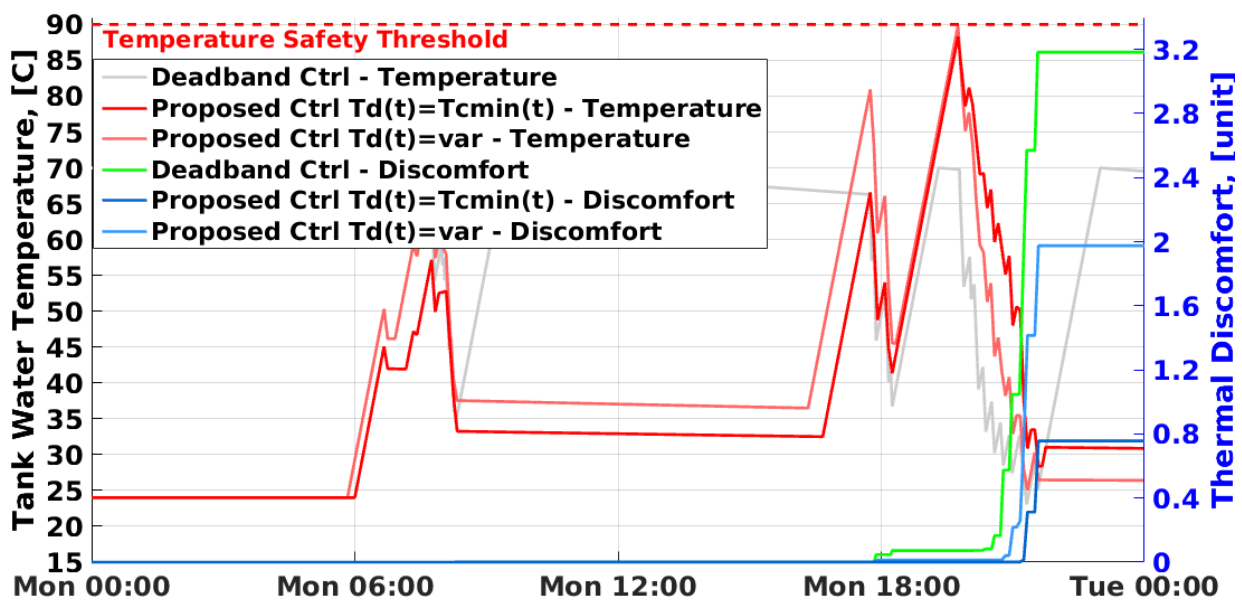

Figure 5.17: Typical working day - Thermal comfort under maximum comfort setting.

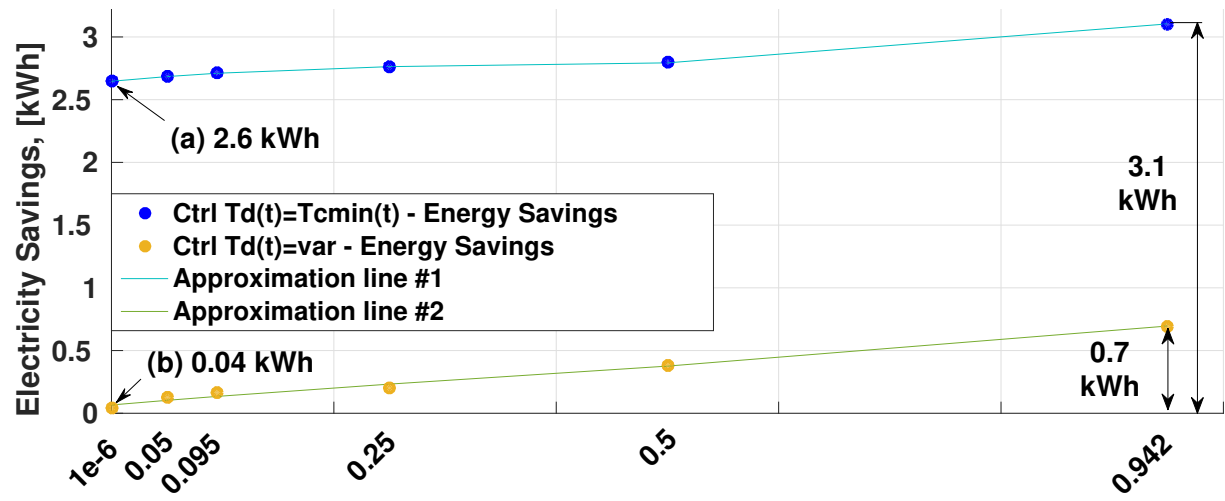

Insulation UA-factor, [W/K]

Figure 5.18: Typical working day - Electricity savings under different insulation.

reduction of the demanded energy $E_{\mathrm{d}}(t)$ permitted by the user thermal tolerance. As such, the electricity savings are equal to that amount of that reduced $E_{\mathrm{d}}(t)$, if the insulation of the WH is close to ideal (ref. (a) in Figure 5.18). By contrast, the electricity savings are only determined by the amount of the reduced heat losses in the control option (ii). The non-zero electricity savings under $U A=10^{-6}$ (ref. (b) in Figure 5.18) can be explained by a lower thermal comfort level than in the deadband control, and as a result, the reduced demanded energy $E_{\mathrm{d}}(t)$ as shown in Figure 5.19(c).

To validate the quality of the solutions obtained by the energy model, we calculated the energy balances between input and output of the hot water system. By subtracting the equation of the energy conservation law for the deadband control from the analogous 
equation in case of the proposed control, one can get the following expression:

$$
\Delta E_{\text {sys }}^{*}(t)=E \mathrm{e}_{\text {save }}(t)-\Delta E_{\mathrm{d}}(t)-\Delta Q_{\text {loss }}(t)
$$

where $\Delta E_{\text {sys }}^{*}(t)$ denotes a difference between the changes of the internal energy of the system $\Delta E_{\text {sys }}^{*}(t)=\Delta E_{\text {sys, deaband }}-\Delta E_{\text {sys, prop.ctrl. }}$ at time $t, \Delta E_{\mathrm{d}}(t)$ stands for difference between the amounts of the demanded thermal energy $\Delta E_{\mathrm{d}}(t)=E_{\mathrm{d} \text {, deaband }}(t)-E_{\text {d,prop.ctrl. }}(t), \Delta Q_{\text {loss }}(t)$ is a difference between the amounts of heat losses $\Delta Q_{\text {loss }}(t)=Q_{\text {loss,deaband }}(t)-Q_{\text {loss,prop.ctrl. }}(t)$.

By plugging the simulation results into Equation 5.13 one can (1) inspect the quality of the solutions in terms of the energy conservation law fulfillment, and (2) compare the energy shares. The shares of input and output energy into/from the considered hot water system are painted in green and pink colors in Figure 5.19, respectively. The change of the internal energy of water in the WH tank is shown in blue. By summing up the values of all the energy shares shown in Figure 5.19 one can obtain zero, meaning that the energy conservation law holds. Note that value of $\Delta E_{\text {sys }}^{*}(t)$ is depicted with a negative sign.

Since the control option (i) allows for the electricity savings due to both the reduced heat losses $\Delta Q_{\text {loss }}$ and the reduced demanded energy $\Delta E_{\mathrm{d}}$ (Section 5.4.2), the electricity savings are non-zero in case of $U A=1 e^{-6}$, as shown in Figure 5.19(c) (on the left). These savings are determined by the reduced demanded energy $\Delta E_{\mathrm{d}}$. When comparing the case of $U A=0.942$ with the case $U A=1 e^{-6}$ for the control option (i) (Figure 5.19(a) vs. Figure 5.19(c)) one could expect a theoretical decrease of the energy savings by the amount of the reduced heat losses $(0.66 \mathrm{kWh})$. However, the electricity savings decreased by only $\Delta E e_{\text {save }}=3.1-2.64=0.46[\mathrm{kWh}]$. This difference of $0.66-0.46=0.2[\mathrm{kWh}]$ can be clarified by the greater amount of $E_{\mathrm{d}}$ and consequently by the increased thermal user comfort level provided by the control option (i) in case of $U A=0.942$ as opposed to the case of $U A=1 e^{-6}$.

As one can see in Figure 5.19 (on the right), the electricity savings achieved in case of the option (ii) are comparable to the reduced amounts of the heat losses, as expected. A higher comfort level under $U A=0.942$ in Figure 5.19(a) results from the increased demanded energy $E_{\mathrm{d} \text {,prop.trl. }}$. The non-zero electricity savings under $U A=1 e^{-} 6$ in Figure 5.19(c) (on the right) originate from the reduced amount of the demanded energy $E_{\text {d,prop.ctrl. }}$ than in case of the deadband control in our simulations, although such a reduction has resulted in a small thermal discomfort for the user. By making the demanded energy difference $\Delta E_{\mathrm{d}}$ smaller (e.g., by applying a smaller step size $\Delta t$ on the intra-day timescale), one can get the user thermal discomfort closer to a zero.

In our simulations, the end-user accepted deep tap water temperature drops during the WAs and about $80 \%$ of the electricity savings $(U A=0.942)$ in case of the control (i) $T_{\mathrm{d}}(t) \approx T_{\text {c.min }}(t)$ resulted from the fact that flow control exploited the existing user thermal tolerance, i.e. provided the demanded energy $E_{\mathrm{d}}$ at the minimum, yet comfortable to the user, level. Therefore, it is reasonable to ask how energy savings will change when the user has a smaller or no thermal tolerance. In terms of our user thermal comfort model, a smaller thermal tolerance means a smaller area between the user comfort boundaries $T_{\text {c.min }}$ and $T_{\text {c.max }}$, i.e. $T_{\text {c.min }}(t) \rightarrow T_{\text {c.max }}(t)$. In that case, the control option (i) has to provide the increased demanded energy $E_{\mathrm{d}}(t)$ to satisfy the elevated $T_{\mathrm{c} \text {.min }}(t)$ at the expense of the electricity savings. It is useful to know under what user comfort tolerance the option (i) becomes worse than (ii) energy-wise. To answer this question, we ran simulations of the same WA profile while gradually increasing $T_{\text {c.min }}$ up to $T_{\text {c.max }}$ during WAs. 


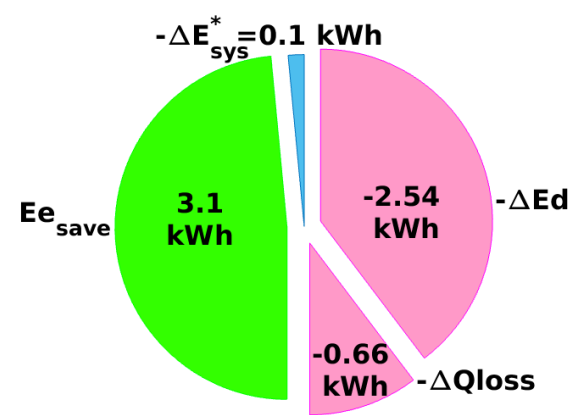

Comfort Increase $=\mathbf{0} .15$ unit

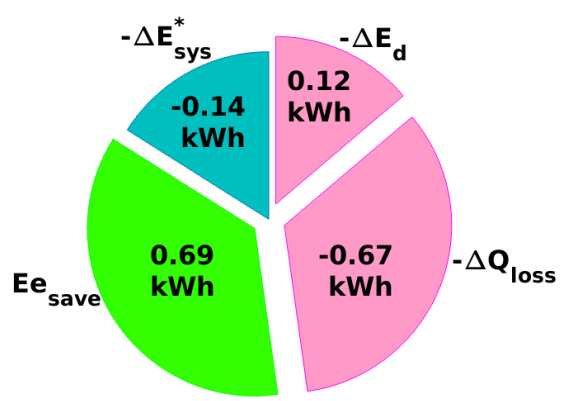

Comfort Increase $=\mathbf{0 . 0 2}$ unit

(a) $\mathrm{UA}=0.942$

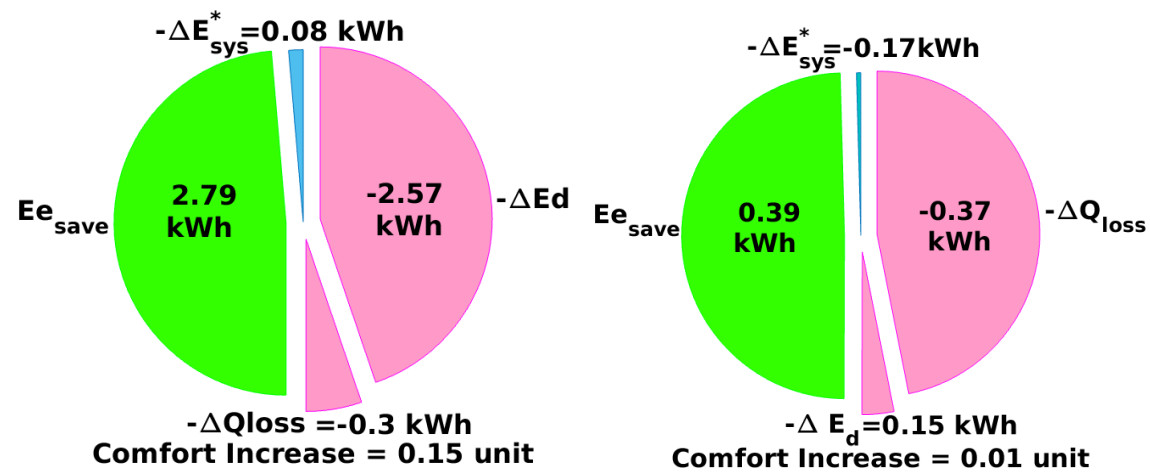

(b) $\mathrm{UA}=0.095$
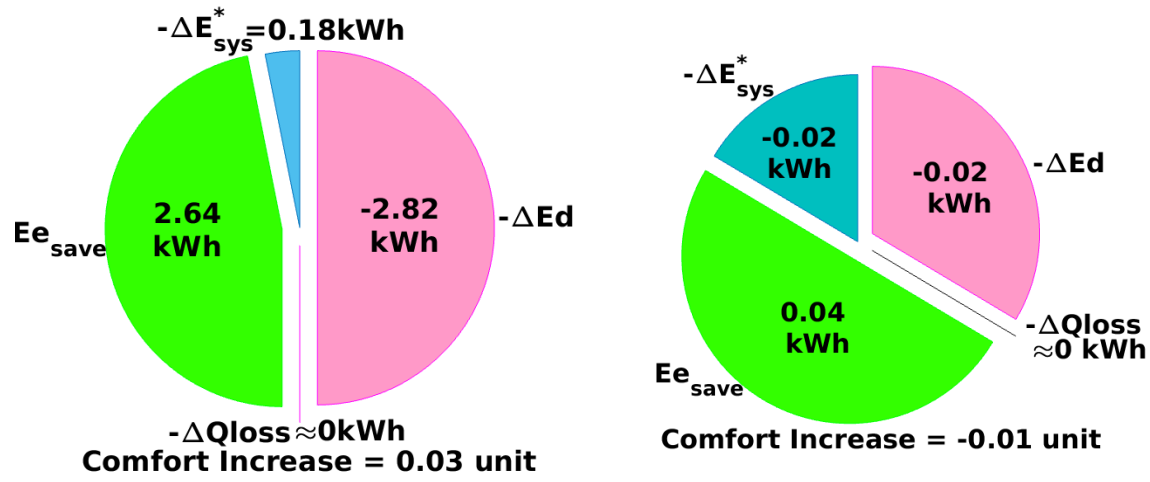

(c) $\mathrm{UA}=1 e^{-6}$

Figure 5.19: Energy balances. On the left - (i) $T_{\text {comf.min }}(t)=T_{\text {comf.max }}(t)$, on the right - (ii) $T_{\mathrm{d}}(t)=v a r$.

The resulting electricity savings are depicted vs. the share of the initial thermal comfort 
zones $\left[T_{\text {c.min }}, T_{\text {c.max }}\right]$ during WAs in Figure 5.20 . Specifically, $100 \%$ share of the initial comfort zone corresponds to the scenario in Figure 5.14, while $0 \%$ share corresponds to $T_{\text {c.min }}(t)=T_{\text {c.max }}(t)$ during the WAs. As before, we aimed at providing the user thermal comfort as close as possible to that brought by the deadband control.

As expected, the control option (i) can become worse than the option (ii) in terms of the electricity savings with the increase of $T_{\text {c.min }}(t)$. More specifically, the electricity savings gradually drop in case of the option (i) because of the required higher demanded energy $E_{\mathrm{d}}(t)$. By contrast, the flat line in case of (ii) $T_{\mathrm{d}}(t)=$ var can be explained by the fact that the demanded energy supplied to the user remains approximately equal to the demanded energy provided by the deadband control, i.e. the control option (ii) does not change the demanded energy, but rather affects the heat losses. In case of $0 \%$ share of the initial comfort zone, the option (i) becomes by 0.25 [kWh] less advantageous than (ii) energy wise. To get a better insight into the driving factor(s) of such change, we calculated the energy balances for the two comfort levels, namely for $0 \%$ and $30 \%$ shares of the initial user comfort zone. The obtained energy balances are shown in Figure 5.21 and Figure 5.22.

From Figure 5.21 and Figure 5.22 it follows that the demanded energy $E_{\mathrm{d}}(t)$ provided in the option (i) exceeds by $0.63[\mathrm{kWh}] E_{\mathrm{d}}(t)$ in (ii) under the comfort share of $0 \%$. As opposed, the demanded energy $E_{\mathrm{d}}(t)$ provided by (ii) exceeds $E_{\mathrm{d}}(t)$ in the case of (i) by 0.23 [kWh] under the $30 \%$ comfort share.

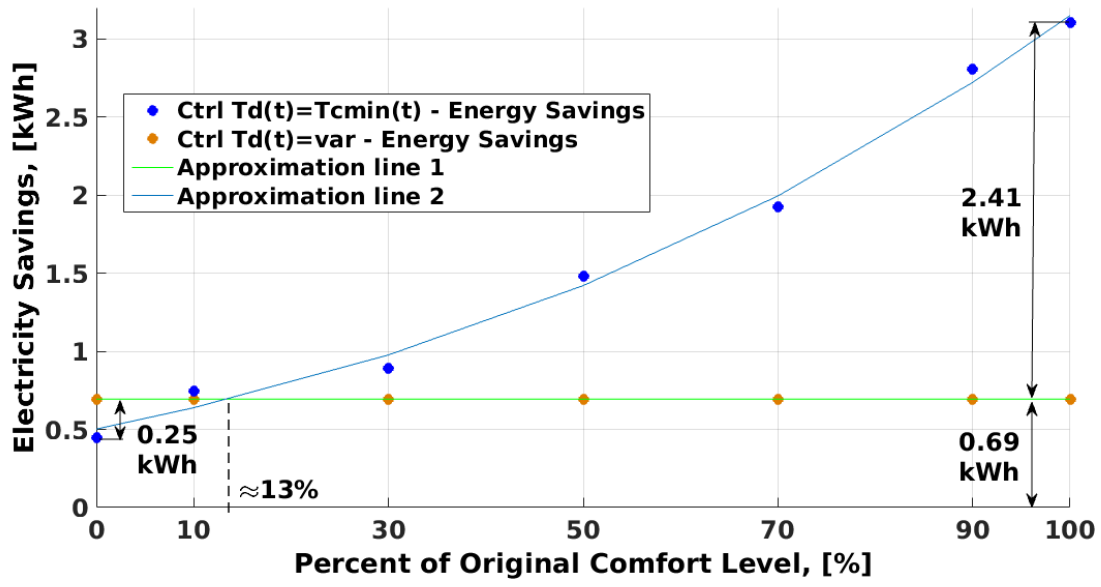

Figure 5.20: Typical working day - Electricity savings vs. user thermal tolerance (as percent of comfort zone). 


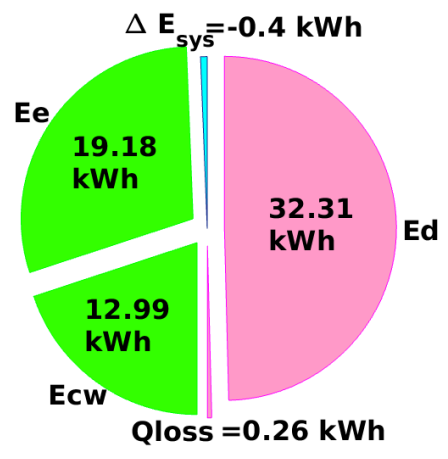

(a)

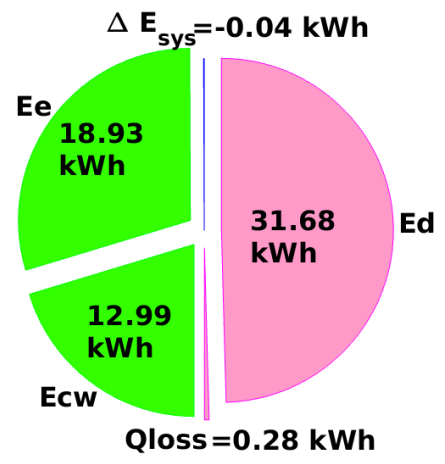

(b)

Figure 5.21: Energy balances at $0 \%$ of initial comfort zone (a) option (i) $T_{\mathrm{d}}(t)=T_{\text {c.min }}(t)$, (b) option (ii) $T_{\mathrm{d}}(t)=v a r$.

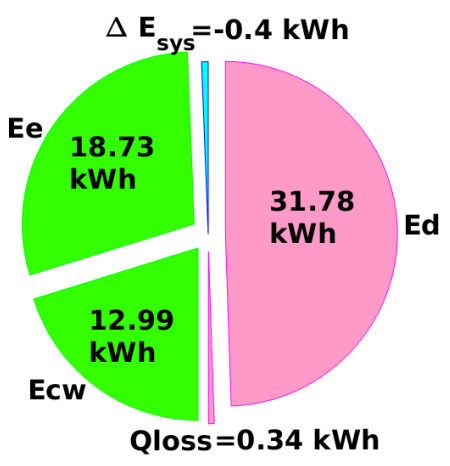

(a)

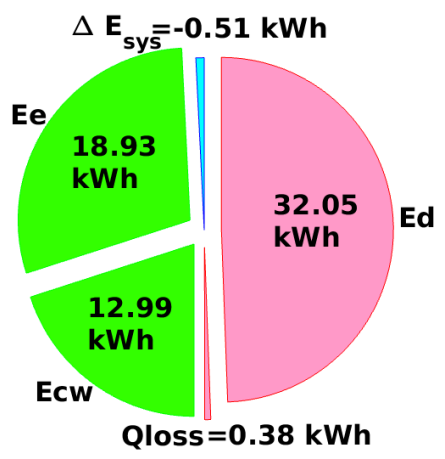

(b)

Figure 5.22: Energy balance - 30\% of initial comfort zone (a) option (i) $T_{\mathrm{d}}(t)=T_{\text {c.min }}(t)$, (b) option (ii) $T_{\mathrm{d}}(t)=$ var. 


\section{Simulation Results for Multiple Days}

The simulation results for an extended period of eight days are summarized in Table 5.1.

Table 5.1: Simulation Results for Extended Period.

\begin{tabular}{|c|c|c|c|c|c|c|c|c|c|c|c|c|}
\hline \multirow{3}{*}{ Period } & \multicolumn{8}{|c|}{ Proposed System } & \multirow{2}{*}{\multicolumn{4}{|c|}{ Deadband Control }} \\
\hline & \multicolumn{4}{|c|}{$T_{\mathrm{d}}(t)=T_{\text {c.min }}(t)^{\mathrm{a}}$} & \multicolumn{4}{|c|}{$T_{\mathrm{d}}(t)=v a r^{\mathrm{b}}$} & & & & \\
\hline & $\begin{array}{c}E_{\mathrm{e}} \\
{[\mathrm{kWh}]}\end{array}$ & $\begin{array}{c}E_{\mathrm{d}} \\
{[\mathrm{kWh}]}\end{array}$ & $\begin{array}{l}Q_{\text {loss }}, \\
{[\mathrm{kWh}]}\end{array}$ & $\begin{array}{c}D_{\mathrm{T}}, \\
\text { [unit] }\end{array}$ & $\begin{array}{c}E_{\mathrm{e}} \\
{[\mathrm{kWh}]}\end{array}$ & $\begin{array}{c}E_{\mathrm{d}} \\
{[\mathrm{kWh}]}\end{array}$ & $\begin{array}{c}Q_{\text {loss }}, \\
{[\mathrm{kWh}]}\end{array}$ & $\begin{array}{c}D_{\mathrm{T}}, \\
\text { [unit] }\end{array}$ & $\begin{array}{c}E_{\mathrm{e}}, \\
{[\mathrm{kWh}]}\end{array}$ & $\begin{array}{c}E_{\mathrm{d}} \\
{[\mathrm{kWh}]}\end{array}$ & $\begin{array}{c}Q_{\text {loss }}, \\
{[\mathrm{kWh}]}\end{array}$ & $\begin{array}{c}D_{\mathrm{T}}, \\
\text { [unit] }\end{array}$ \\
\hline D1-D7 & 141.12 & 249.76 & 1.78 & 26.85 & 160.73 & 269.35 & 2.04 & 28.59 & 161.33 & 267.51 & 5.85 & 28.75 \\
\hline D2-D8 & 136.49 & 244.28 & 2.05 & 25.65 & 156.74 & 264.38 & 2.17 & 27.47 & 159.79 & 262.59 & 5.89 & 27.48 \\
\hline
\end{tabular}

(a) corresponds to the control option (i) $T_{\mathrm{d}}(t) \approx T_{\text {c.min }}(t)$.

(b) stands for the control option (ii) $T_{\mathrm{d}}(t)=$ var.

Note! The green color signifies improvement of a parameter as compared to other values in the same row. The light and dark colors indicate the lowest and the biggest improvement of a value in one row, respectively. The demanded energy $E_{\mathrm{d}}$ is present for a reference purpose only.

The percentages of the corresponding changes of the electricity consumption, heat losses and user thermal comfort are listed in Table 5.2.

Table 5.2: Simulation Results for Extended Period - Percentages.

\begin{tabular}{|c|c|c|c|c|c|c|}
\hline \multirow{3}{*}{ Period } & \multicolumn{5}{|c|}{ Proposed System } \\
\cline { 2 - 7 } & \multicolumn{3}{|c|}{$T_{\mathrm{d}}(t)=T_{\text {c.min }}(t)$} & \multicolumn{3}{c|}{$T_{\mathrm{d}}(t)=v a r$} \\
\cline { 2 - 7 } & $E_{\mathrm{e}, \text { save, }[\%]}$ & $\Delta Q_{\text {loss }},[\%]$ & $\Delta D_{\mathrm{T}},[\%]$ & $E_{\mathrm{e}, \text { save, }[\%]}$ & $\Delta Q_{\text {loss }},[\%]$ & $\Delta D_{\mathrm{T}},[\%]$ \\
\hline D1-D7 & 12.53 & 69.57 & 6.61 & 0.37 & 65.13 & 0.56 \\
\hline D2-D8 & 14.58 & 65.20 & 6.66 & 1.91 & 63.16 & $\approx 0$ \\
\hline
\end{tabular}

The simulation results for the control option (i) $T_{\mathrm{d}}(t) \approx T_{\text {c.min }}(t)$ are present in Figure 5.23 to Figure 5.25.

One can see in Figure 5.23 that the control option (i) is capable to attain electricity savings both in case of the "cold" start $T(0)=15^{\circ} \mathrm{C}(\mathrm{D} 1-\mathrm{D} 7)$ and in case of the "hot" start $T(0)=36.28^{\circ} \mathrm{C}(\mathrm{D} 2-\mathrm{D} 8)$. While the electricity savings are equal to $23.30[\mathrm{kWh}]$ during the period D2-D8, electricity savings constitute only 20.22 [kWh] for D1-D7. This difference of $3.08[\mathrm{kWh}]$ is partly due to the necessity to heat the water from the lower temperature on D1 as compared to D2 and also because of a different hot water usage profile on D8. The effect of different initial tank water temperatures on the electricity savings by the end of D1 in case of (i) is shown in Figure 5.26. 


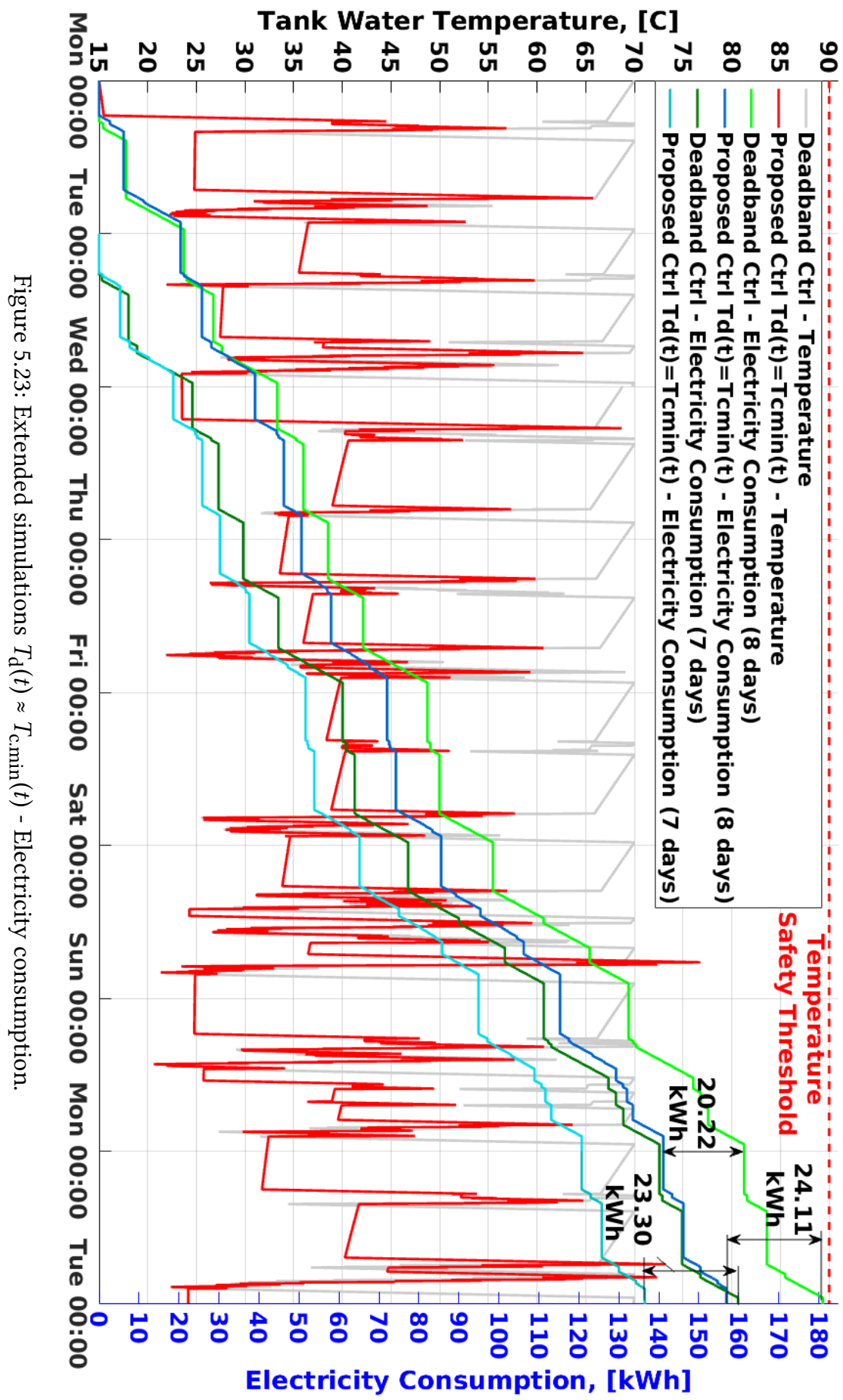




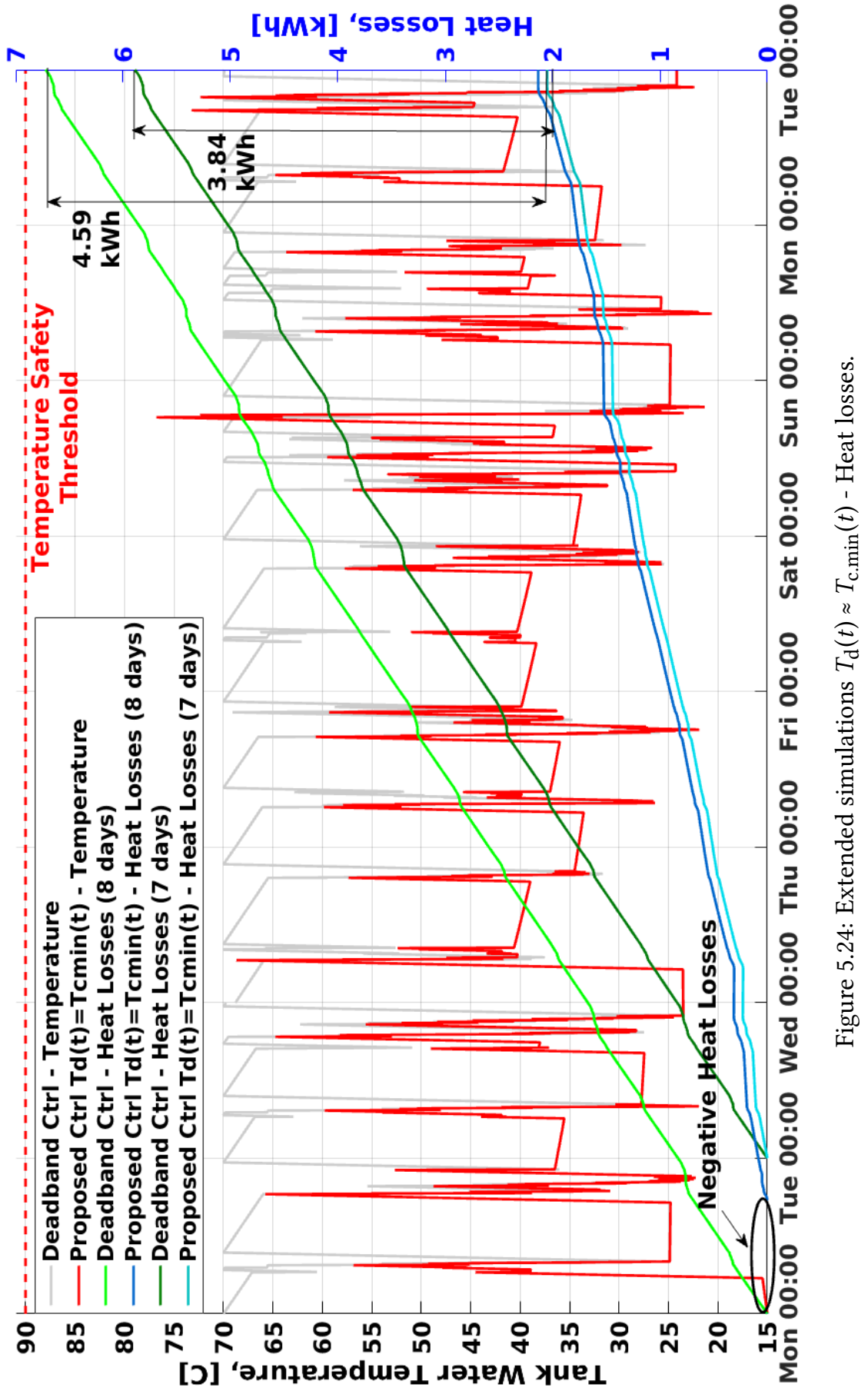




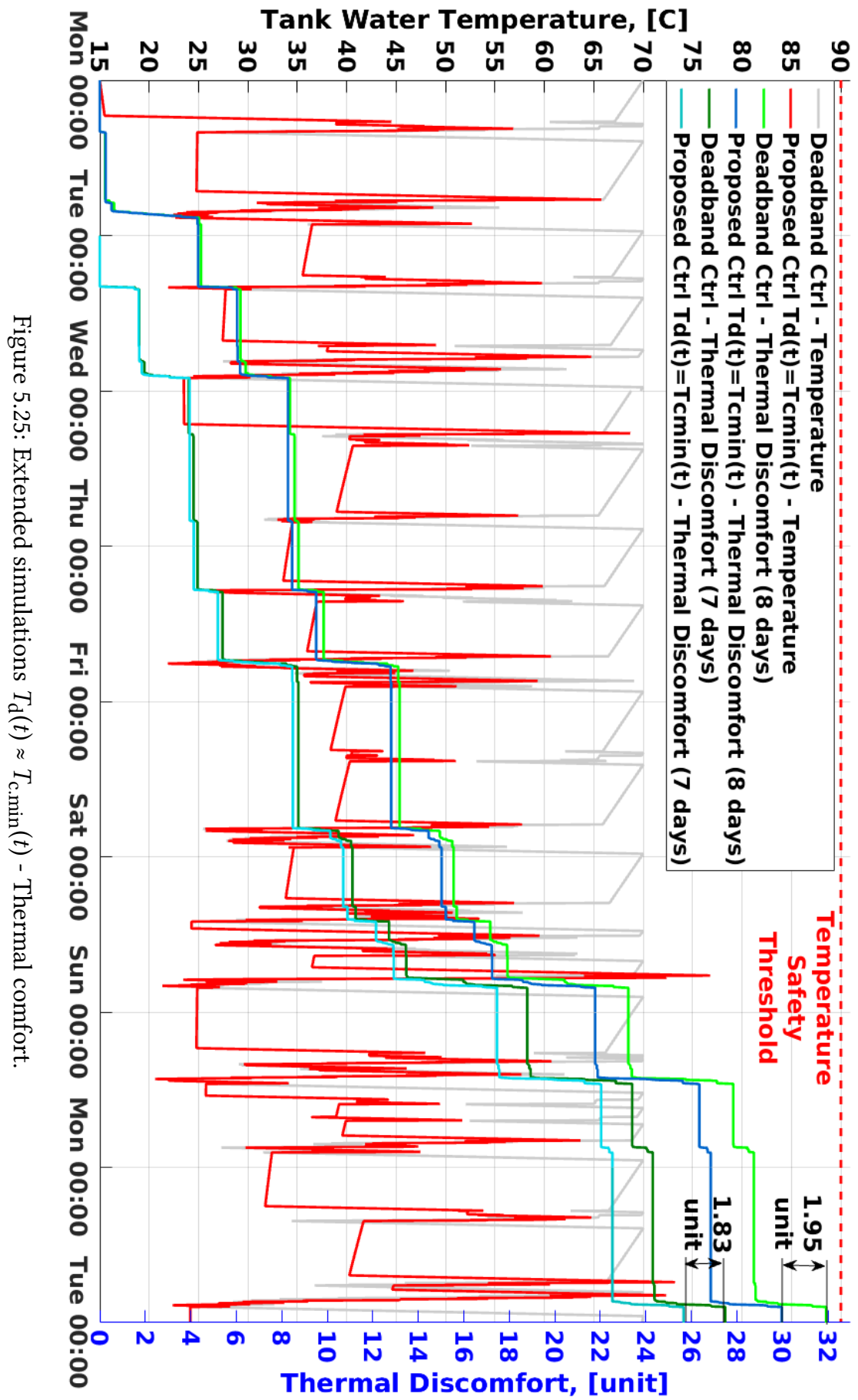




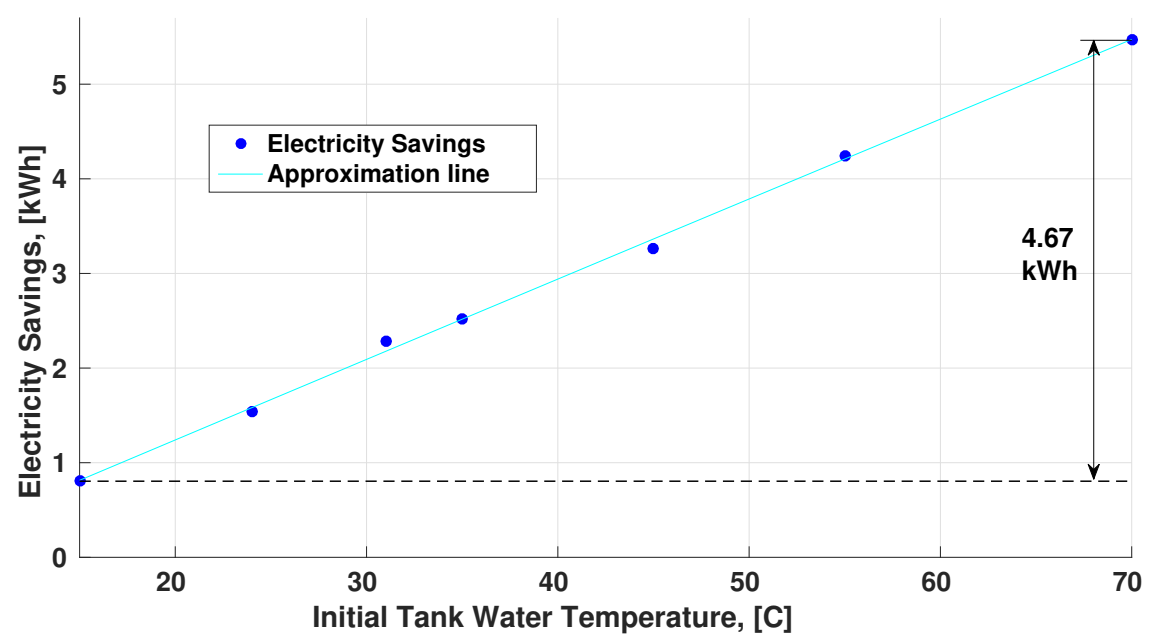

Figure 5.26: Control option (i) $T_{\mathrm{d}}(t) \approx T_{\text {c.min }}(t)$ - Effect of initial tank water temperature on electricity savings (D1).

Figure 5.26 represents an approximately linear relationship between the energy savings and the initial tank water temperature on D1. It follows from Figure 5.26 that if the control option (i) were started at the initial tank water temperature $T(0)=70^{\circ} \mathrm{C}$, it would become $4.67[\mathrm{kWh}]$ more advantageous than in the case of $T(0)=15^{\circ} \mathrm{C}$ shown in Figure 5.23. The electricity savings by the end of D1 would then account for $24.11[\mathrm{kWh}]-23.30[\mathrm{kWh}]+$ $4.67[\mathrm{kWh}]=5.48[\mathrm{kWh}]$. This "hidden" electrical energy reserve of $4.67[\mathrm{kWh}]$ not accounted for in our comparison could be used for a sanitary cycle to prevent bacteria Legionella, if needed.

As one can find in Figure 5.24, the heat losses are reduced by 4.07 [kWh] and by 3.84 [kWh] by the end of D1-D7 and D2-D8 periods, respectively. Therefore, the contribution of the reduced demanded energy $E_{\mathrm{d}}$ into the electricity savings accounts for $E_{\mathrm{e}}$, save $-\Delta Q_{\text {loss }}=$ $20.22-4.07=16.15$ [kWh] (equivalent to $79.87 \%$ of the electricity saved) for D1-D7, and 19.46 [kWh] (83.52\% of the electricity savings) for D2-D8, which indicates that the user thermal comfort tolerance is the main reason of the electricity savings in the control option (i) in our simulations.

As Figure 5.25 shows, the thermal comfort level provided by (i) exceeds the thermal comfort level furnished by the deadband control by 1.9 [unit] and by 1.83 [unit] in scenarios D1-D7 and D2-D8, respectively.

The simulation results for the control option (ii) $T_{\mathrm{d}}(t)=$ var are illustrated in Figure 5.27Figure 5.29. 


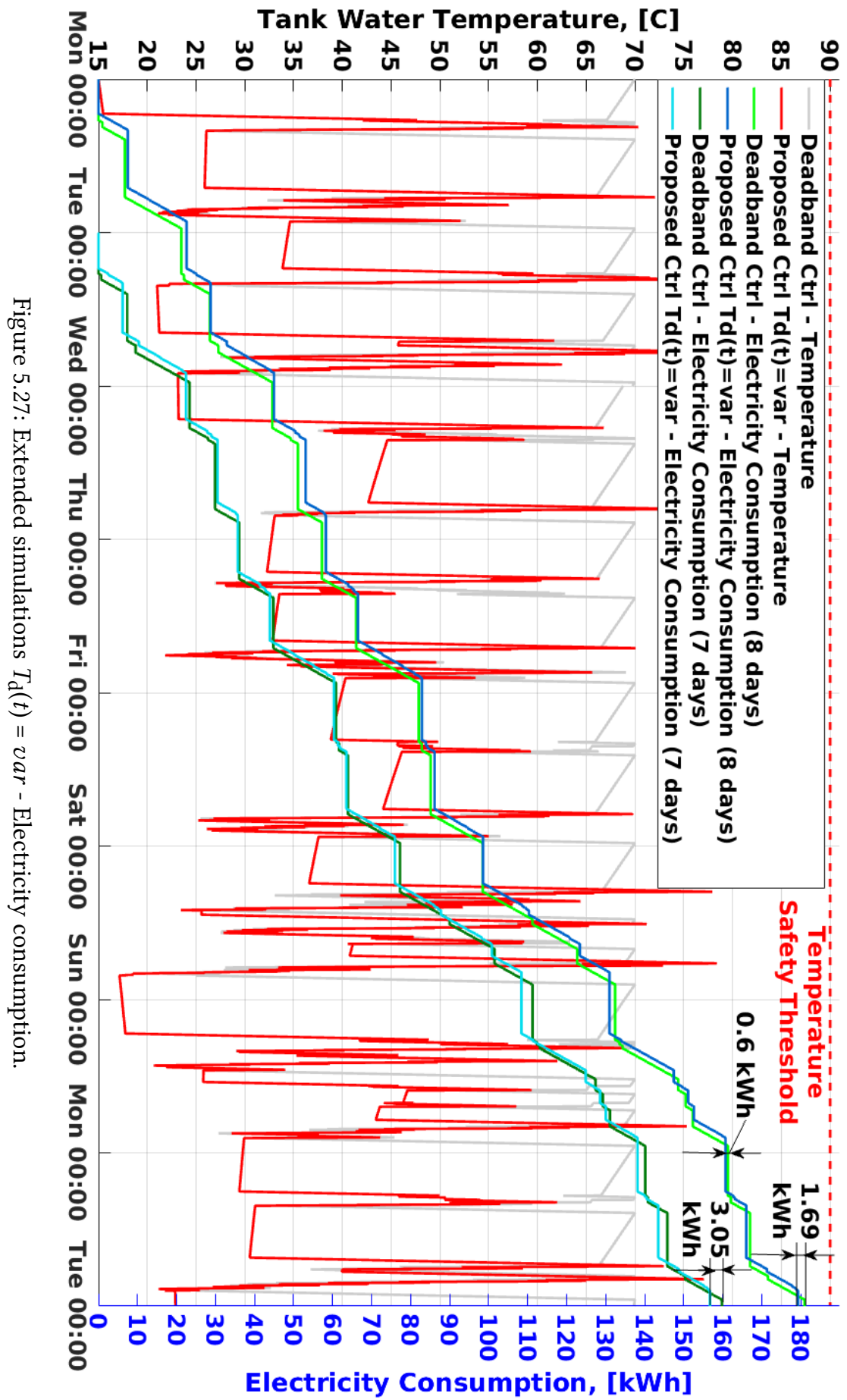




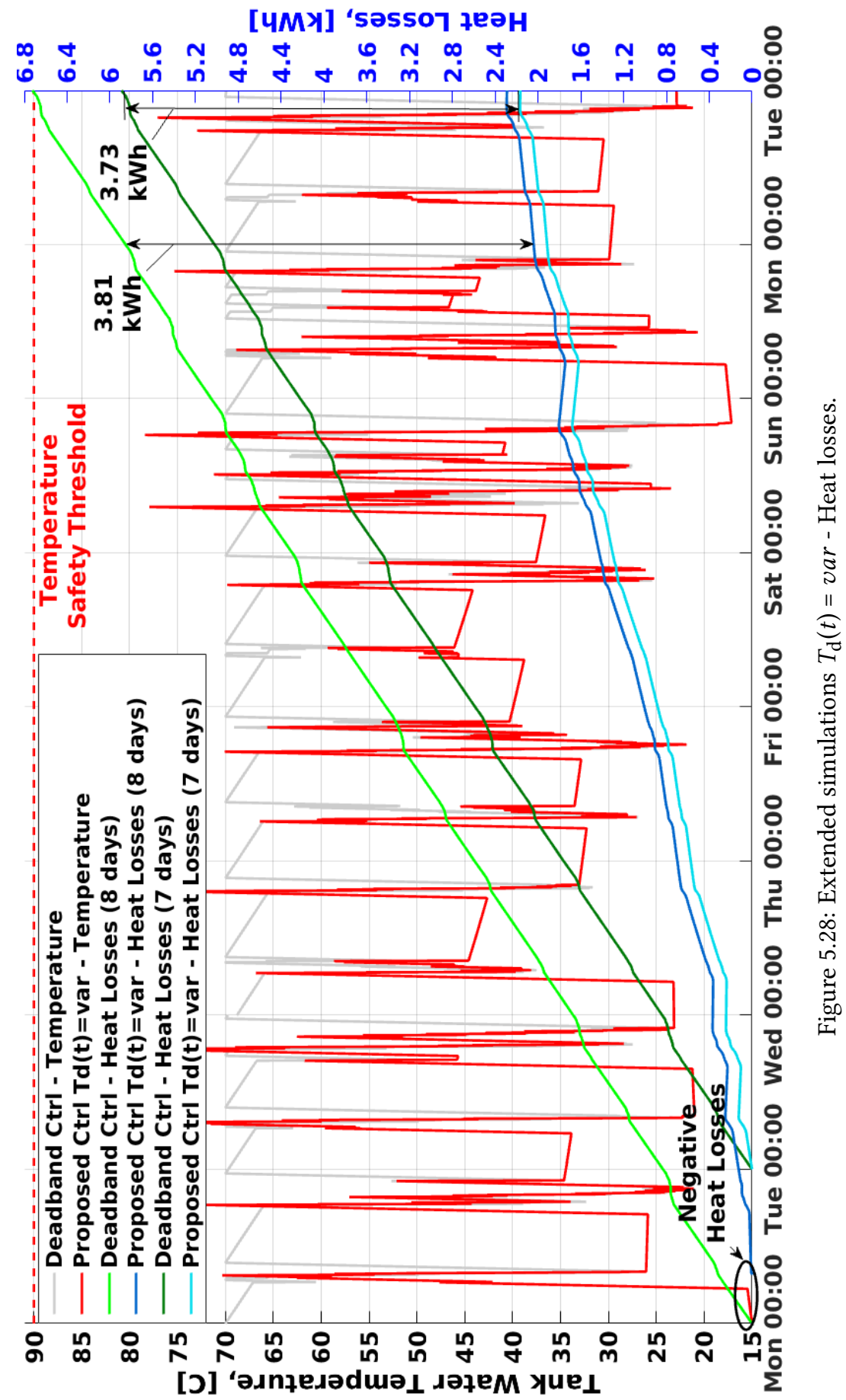




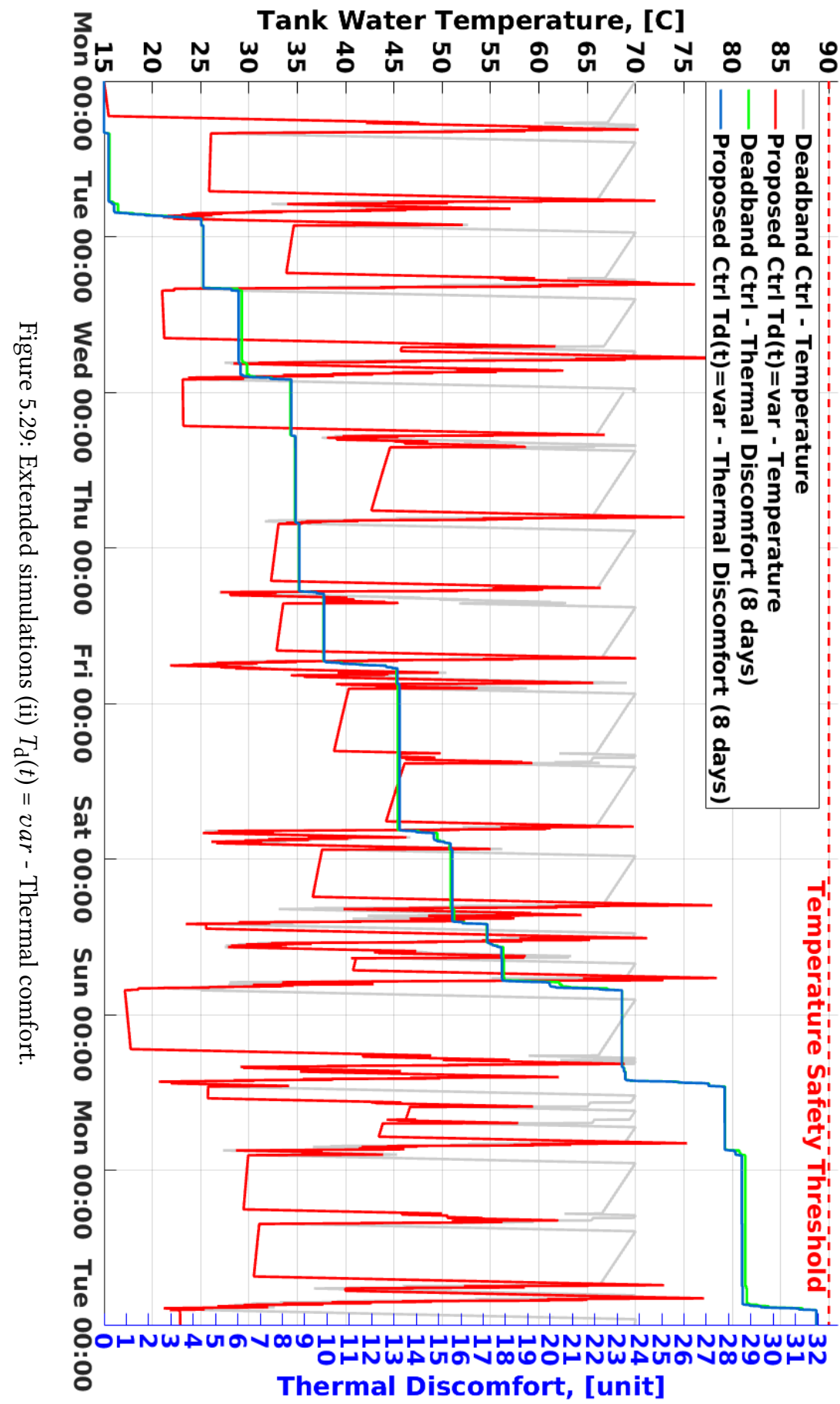


Similar to the control option (i), the simulation results for (ii) $T_{\mathrm{d}}(t)=v$ ar during a period D1-D7 ("cold" start) exhibit significantly lower 0.6 [kWh] electricity savings as against $3.05[\mathrm{kWh}$ ] electricity savings at the end of the simulation period D2-D8 ("hot" start). This is partly due to a different hot water profile on D8 and partly due to the different initial tank water temperatures at the beginning of D1-D7 period $\left(15^{\circ} \mathrm{C}\right)$ and $\mathrm{D} 2-\mathrm{D} 8$ period $\left(34.45^{\circ} \mathrm{C}\right)$. Noteworthy, the electricity savings achieved during the day D8 $(1.69[\mathrm{kWh}]-0.6[\mathrm{kWh}]=1.09[\mathrm{kWh}])$ significantly contribute $(35.74 \%)$ to the total electricity savings within a period D2-D8.

Unlike the control option (i), there were simulation days where (ii) demonstrated negative electricity savings. For example, by the end of D1 the deadband control outperforms (ii) by $1.36\left[\mathrm{kWh}\right.$, which can be clarified by initial tank water temperature equal to $15^{\circ} \mathrm{C}$ applied in simulations of (ii). Also, the electricity consumption in case of (ii) is higher than that of the deadband control by $0.56[\mathrm{kWh}]$ during D3 and by 0.84 [kWh] during D7. This comes from the higher thermal comfort levels in case of (ii), which account for 0.02 [unit] during D3 and 0.14 [unit] during D7. By aligning the comfort levels of the proposed control to the deadband control more accurately, this side effect can be remedied.

As it can be seen from the graphs of the heat losses in Figure 5.28, the electricity savings $E_{\text {save }}(t)$ are lagging behind the difference $\Delta Q_{\text {loss }}(t)$ between the heat losses during the proposed control and the deadband control for both the periods D1-D7 and D2-D8. More specifically, the mismatch between $E_{\text {save }}(\mathrm{D} 1-\mathrm{D} 7)=0.6[\mathrm{kWh}]$ and $\Delta Q_{\text {loss }}(\mathrm{D} 1-\mathrm{D} 7)=3.81$ [kWh] during the period D1-D7 accounts for 3.21 [kWh], while the mismatch for the period D2-D8 is only 0.68 [kWh]. Although it is expected that (ii) should provide electricity savings comparable to the amount of the heat losses reduced, the discovered mismatches originate from the higher thermal comfort levels brought by the proposed control in our simulations. The total thermal comfort improvement delivered by (ii) by the end of the period D1-D7 accounts for 0.16 [unit] together with the increased by 1.84 [kWh] demanded energy $E_{\mathrm{d}}(\mathrm{D} 1-\mathrm{D} 7)$ as compared to the deadband control (see Table 5.1). The thermal comfort is improved by 0.01 [unit] along with the increased by 1.79 [kWh] at the end of the period D2-D8. As one can notice, the thermal comfort improvement of 0.01 [unit] during the period D2-D8 is much lower than that during the period D1-D8 (0.16 [unit]), which results in a smaller mismatch between the electricity savings $E_{\text {save }}(\mathrm{D} 2-\mathrm{D} 8)$ and heat loss reduction $\Delta Q_{\text {loss }}(\mathrm{D} 2-\mathrm{D} 8)(0.68[\mathrm{kWh}])$ compared with the period D1-D7 (3.21 [kWh]).

After simulations obtained for an extended period, we can estimate the average electricity savings for working days and weekends in case of the control options (i) and (ii). The daily averages obtained based on extended period D2-D8 are listed in Table 5.3 and Table 5.4. All the percentages shown in Table 5.3 and Table 5.4 are calculated as an average of percentages of changed values of the corresponding parameters.

The control options (i) and (ii) demonstrate higher average percent of the electricity savings during the working days than during the weekends as can be concluded based on only the percentages in Table 5.3 and Table 5.4. However, as one can find in Table 5.3, the average amount of energy saved during the working days is lower than that for the weekend days, even though the percent of electricity savings is higher. This can be clarified as follows. The number of WAs during the weekend days is greater than that during the working days in our simulations. Those "extra" WAs during the weekends present their own user comfort tolerances and thus allow for additional demanded energy reduction 
Table 5.3: Daily averages - Control option (i) $T_{\mathrm{d}}(t)=T_{\text {c.min }}(t)$.

\begin{tabular}{|c|c|c|c|c|c|c|}
\hline \multirow{2}{*}{ Day Type } & \multicolumn{6}{|c|}{ Proposed System } \\
\cline { 2 - 7 } & \multicolumn{2}{|c|}{$\tilde{E}_{\mathrm{e}, \text { save }}$} & \multicolumn{2}{c|}{$\Delta \tilde{Q}_{\text {loss }}$} & \multicolumn{2}{c|}{$\Delta \tilde{D}_{\mathrm{T}}$} \\
\cline { 2 - 7 } & $\mathrm{kWh}$ & $\%$ & $\mathrm{kWh}$ & $\%$ & unit & $\%$ \\
\hline working & 3.21 & 15.98 & 0.56 & 64.91 & 0.09 & 12.19 \\
\hline weekend & 3.64 & 11.52 & 0.68 & 65.79 & 0.51 & 10.02 \\
\hline
\end{tabular}

Table 5.4: Daily averages - Control option (ii) $T_{\mathrm{d}}(t)=$ var.

\begin{tabular}{|c|c|c|c|c|c|c|}
\hline \multirow{2}{*}{ Day Type } & \multicolumn{6}{|c|}{ Proposed System } \\
\cline { 2 - 7 } & \multicolumn{2}{|c|}{$\tilde{E}_{\mathrm{e}, \text { save }}$} & \multicolumn{2}{c|}{$\Delta \tilde{Q}_{\text {loss }}$} & \multicolumn{2}{c|}{$\Delta \tilde{D}_{\mathrm{T}}$} \\
\cline { 2 - 7 } & $\mathrm{kWh}$ & $\%$ & $\mathrm{kWh}$ & $\%$ & unit & $\%$ \\
\hline working & 0.48 & 2.10 & 0.56 & 64.21 & $\approx 0$ & $\approx 0$ \\
\hline weekend & 0.32 & 0.72 & 0.47 & 60.52 & 0.11 & 1.79 \\
\hline
\end{tabular}

resulting in the higher electricity savings. On the other hand, the electricity consumption during the weekend days surpasses the electricity consumption during the working days (in average by $11.51[\mathrm{kWh}]$ ). As a result, the percentage of electricity saved during the weekend days is lower than that during the working days.

On the contrary, in case of (ii) both the average amount and the average percent of electricity savings is higher for the working days than for the weekend days as shown in Table 5.4. This can be justified by the fact that (ii) provides electricity savings only based on the reduction of the heat losses, regardless of the acceptable user thermal discomfort (aligned to the deadband control). Which means that even though the weekend days contain a bigger number of WAs (in our simulations) with corresponding user thermal comfort tolerances, such tolerances granted by the user are not utilized by (ii). As a result, those "extra" WAs during the weekend days have a negative effect on electricity savings. On the other side, the reduction of the heat losses by (ii) during the relatively long periods of no hot water usage present in the afternoons during the working days make (ii) save more energy during the working days as contrasted to the control option (i) that saves more electricity on profiles that allow for higher reduction of the demanded energy.

Based on Table 5.3 and Table 5.4 it can be concluded that the average electricity savings provided by (i) exceed those rendered by (ii), since in addition to the reduction of the heat losses (i) also reduces the demanded energy $E_{\mathrm{d}}(t)$ allowed by the user thermal comfort tolerance.

The control option (i) in average results in a bigger reduction of the heat losses during the weekend days compared with the option (ii) because of the lower tank water temperature values.

Moreover, the average thermal comfort level delivered by the option (i) during the weekend days is higher than that in case of (ii), although the tank water temperature values are lower. This can be explained by the capability of the flow control supported by (i) to maintain the tap water temperature at the lowest comfortable boundary $T_{\text {c.min }}$ during WAs. In other words, the tap water temperature does not fall uncontrollably as in case of (ii), but 


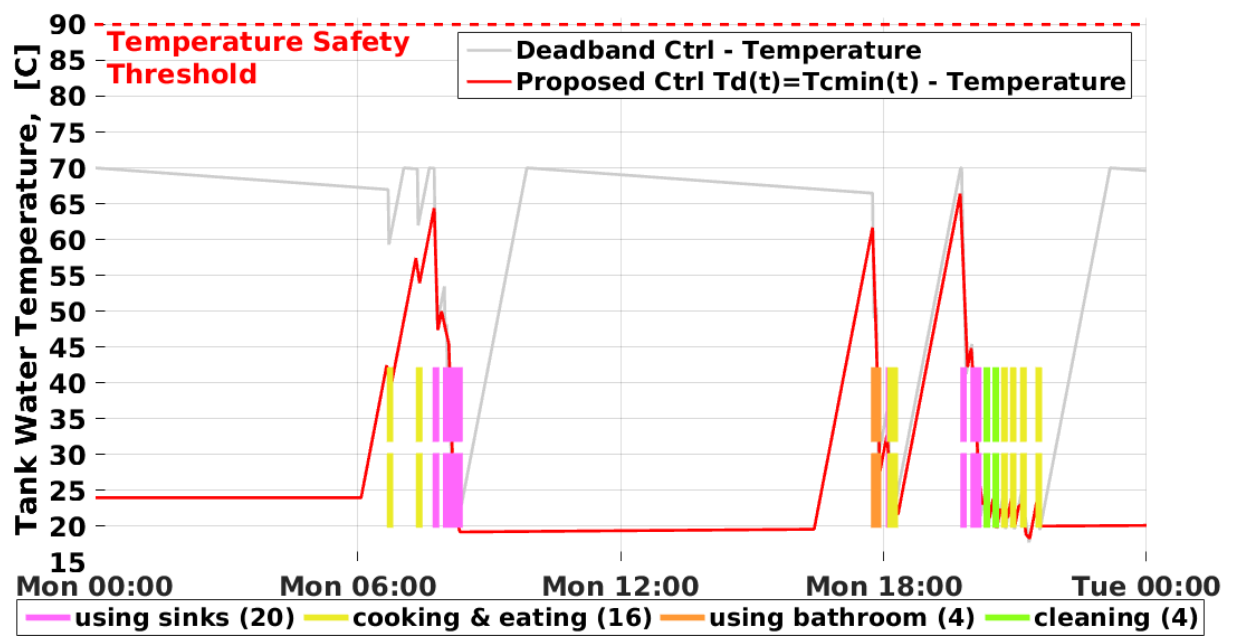

Figure 5.30: Typical working day - Parallel WAs (numbers in braces indicate the number of hot water withrawals).

rather it is maintained within the user comfort zones.

\section{Hot Water Usage Overlapping in Time}

To evaluate the performance of the control option (i) $T_{\mathrm{d}}(t) \approx T_{\text {c.min }}(t)$ ) in case of WAs overlapping in time, we simulated a scenario where two mixer taps were used in parallel. More specifically, we applied two identical hot water consumption profiles with the same time- and comfort-related parameters, i.e. we considered WAs that occur parallel in time. Each of the profiles replicated the profile of a typical working day used in Section 5.4.9. The profiles are illustrated in Figure 5.30.

We compared the simulation results of the energy model with the results of the deadband control under the comparable level of comfort. The simulation results are shown in Figure 5.31 - Figure 5.33.

As one can see in Figure 5.31, the amount of electricity saved by the control option (i) in case of parallel WAs accounts for 2.76 [kWh] (or $11.55 \%$ of electricity used during the deadband control), as against 3.1 [kWh] (15.8\%) electricity savings in case of sequential WAs (Figure 5.13). The energy efficiency of the proposed control thus decreased by 0.34 $[\mathrm{kWh}]$ in comparison with the case of sequential WAs. This can be partly explained by the necessity to heat the water to higher temperatures in order to support more intense hot water withdrawals and by $\approx 0.1[\mathrm{kWh}]$ higher heat losses (Figure 5.32 vs. Figure 5.19(a)(left)) in case of the parallel WAs. Furthermore, the user comfort was provided on the level by 0.13 [unit] higher (Figure 5.33 vs. Figure 5.14) as opposed to the case of sequential WAs. As discussed earlier in Section 5.4.9, a slight difference between the thermal comfort levels in Figure 5.33 is caused by the accuracy of the simulations and can be improved, if necessary.

As it can be seen in Figure 5.34, the reduction of the heat losses accounts for 0.75 [kWh] in the scenario of parallel WAs, which is by $\approx 0.1[\mathrm{kWh}]$ greater than in case of non-parallel 


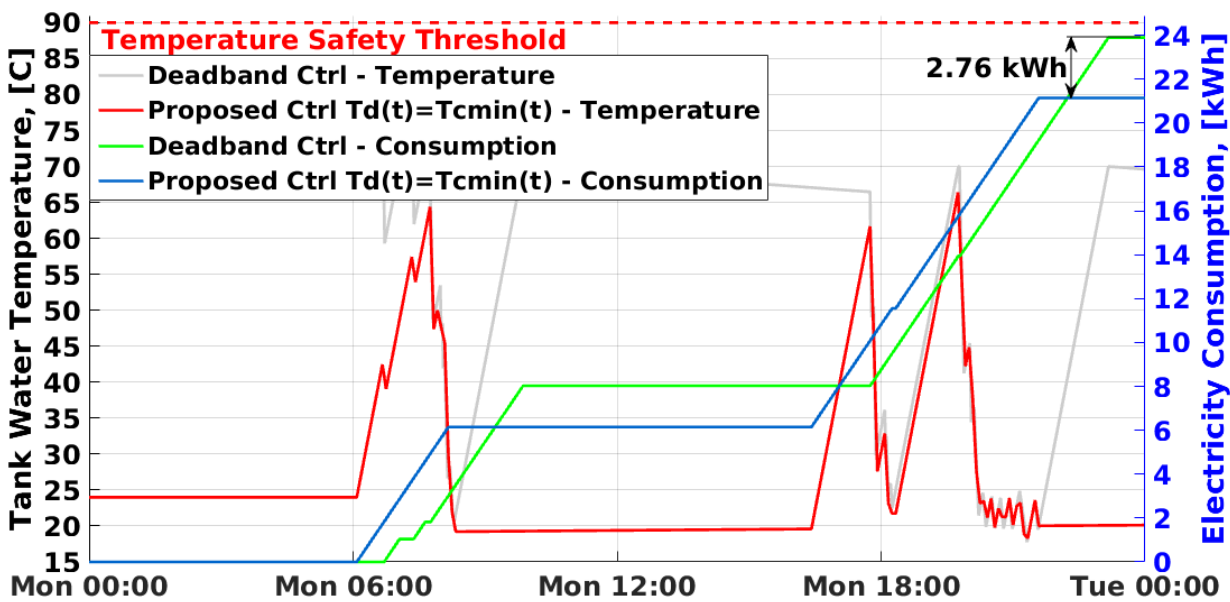

Figure 5.31: Typical working day (parallel WAs) - Electricity savings.

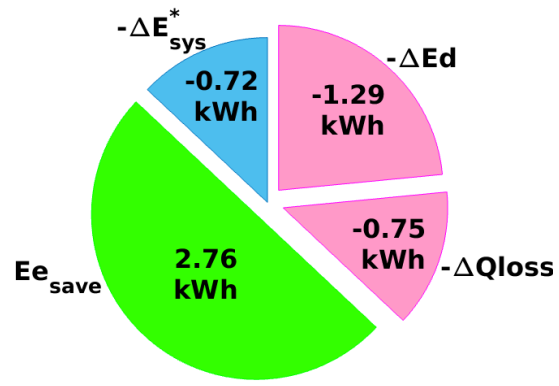

Figure 5.32: Typical working day (parallel WAs) - Energy balance.

WAs (Figure 5.19(a)). The improved heat losses reduction in case of parallel WAs originates from the intense hot water withdrawals that cause the tank water temperature drop below the ambient temperature of $24^{\circ} \mathrm{C}$ and the consequent change of the sign of the heat flux between the $\mathrm{WH}$ and the surroundings.

\section{Simulation Results Summary}

We evaluated the devised energy model for tank electric water heaters based on the simulations for the options (i) $T_{\mathrm{d}}(t) \approx T_{\text {c.min }}(t)$ and (ii) $T_{\mathrm{d}}(t)=v a r$ of the tap water provision.

The simulation results in Section 5.4.9 and Section 5.4.9 show that the energy model is capable to achieve in average $15.98 \%$ and $11.52 \%$ electricity savings under the control option (i) on a working day and a weekend day, respectively. While in case of the option (ii) the model can save the electricity in average by $2.10 \%$ on a working day and $0.72 \%$ on a weekend day. Moreover, the energy model allows for balancing the electricity savings and 


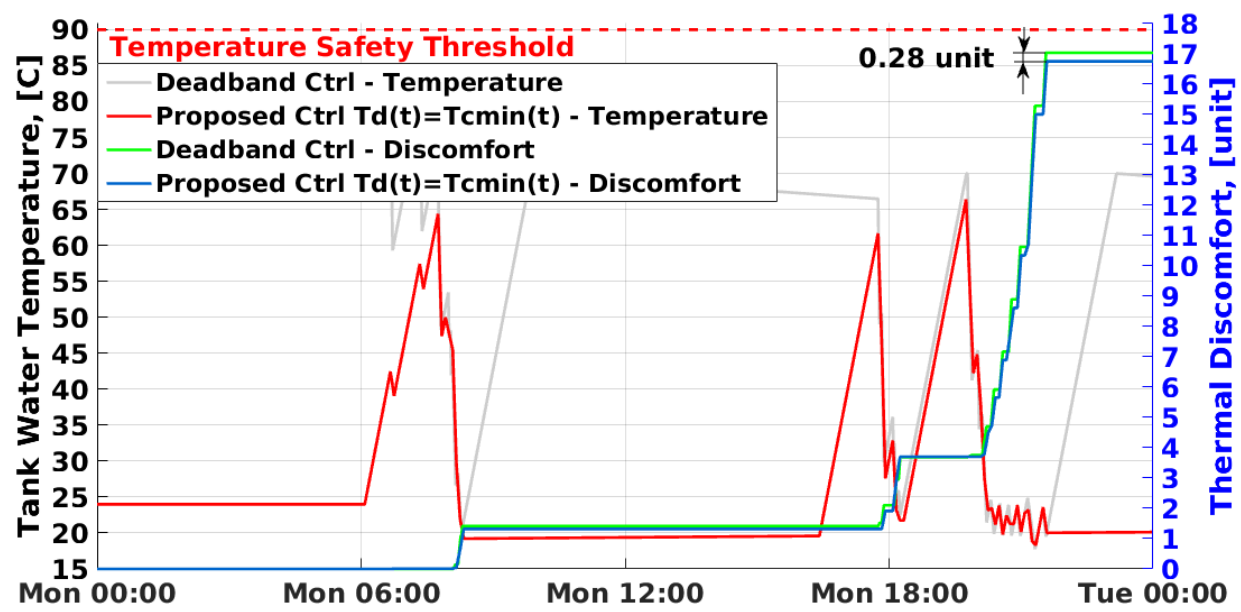

Figure 5.33: Typical working day (parallel WAs) - Thermal comfort.

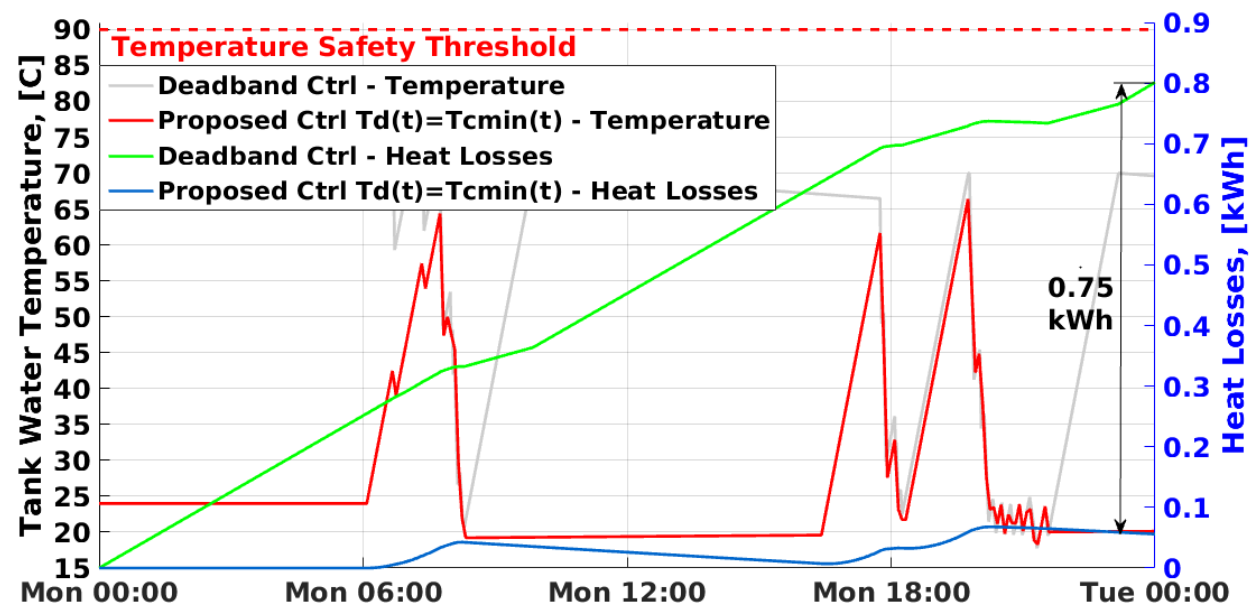

Figure 5.34: Typical working day (parallel WAs) - Heat losses.

user comfort, i.e. the user can opt in favor of higher comfort levels at the cost of higher electricity consumption than that provided by the deadband control, or choose higher electricity savings by sacrificing the personal comfort.

The simulation results for a single day and a weekly period demonstrate the benefits of the control option (i) over the option (ii). However, the benefits are mainly due to the user thermal tolerance allowed by the user and exploited by the control option (i). As it can be found in Figure 5.20, the option (ii) can overplay (i) in the situations where the 
user can barely tolerate the tap water temperature drops. This brings up a question of the user comfort tolerance assessment before a certain option (i) or (ii) is implemented in practice. For example, the choice of the control option (i) or (ii) can be made per WA, rather than for the entire daily profile of WAs (in our simulations). For this, one can estimate the demanded energy and user comfort levels of both the pre-heating and flow control solutions obtained independently for individual WAs prior to the energy model execution. Then, only those WAs whose flow control solutions result in lower demanded energy and better comfort levels than the pre-heating control can be selected for execution under the control option (i), while the rest can be performed under the option (ii). The energy model is flexible to incorporate a mixture of two control options for individual WAs as input.

Assuming a given user thermal tolerance, the efficiency of the proposed energy model, i.e. the option (i), was also evaluated based on a scenario where two mixer taps were used in parallel. The simulation results indicate up to $11.55 \%$ electricity savings under $\approx 1.6 \%$ [unit] comfort improvement achieved by the proposed model as contrasted to the deadband control.

\subsection{Price Model}

In this section, we focus on Problem 3 of the conventional deadband control outlined in Section 5.2, namely the incapability of the deadband control to shift the WH electricity consumption in response to variable electricity prices. To overcome this limitation of the deadband control, we propose a control mechanism that allows for shifting the WH load to the low-price periods of a day in a scenario where multiple WAs during a day.

\subsubsection{Preliminary Discussion and Problem Formulation}

In this section, we elaborate on Problem 3 of the conventional deadband control and formulate the money-comfort problem.

The conventional deadband control does not account for electricity prices, hence cannot suit for automated shifting of the WH load outside the high-price periods. Shifting the WH load by manual adjustment of the thermostat setpoints in response to variable prices does not seem to be a solution either. Residents are typically unaware about the best WH thermostat settings that will maximize money profits and provide a decent level of the thermal comfort at the same time. This task requires the knowledge about the technical parameters of the $\mathrm{WH}$, the expected hot water demand and end-user comfort which are hard to account for. When exercised incautiously, the manual tunning of the WH setpoints can severely downgrade the thermal comfort of occupants.

\section{Effect of Load Shifting on Costs}

Consider a simplified example of single WA and a double-price electricity tariff with a rate difference equal to $€ 0.10$. Suppose, the reduced electricity rate is available from 12:00AM to 7:00AM and a 5-minute water activity (WA) occurs at 8:00AM during the period of high electricity prices. The user desires full comfort satisfaction during the WA. Assume that the $\mathrm{WH}$ is initially cooling down during the 7-hour night period without the electricity consumption as shown in Figure 5.35. The WH turns on at 7:50AM to heat up the water during the regular operation when the tank water temperature hits the thermostat lower setpoint of $65^{\circ} \mathrm{C}$. By initiating the water heating earlier during the low-price night period, 

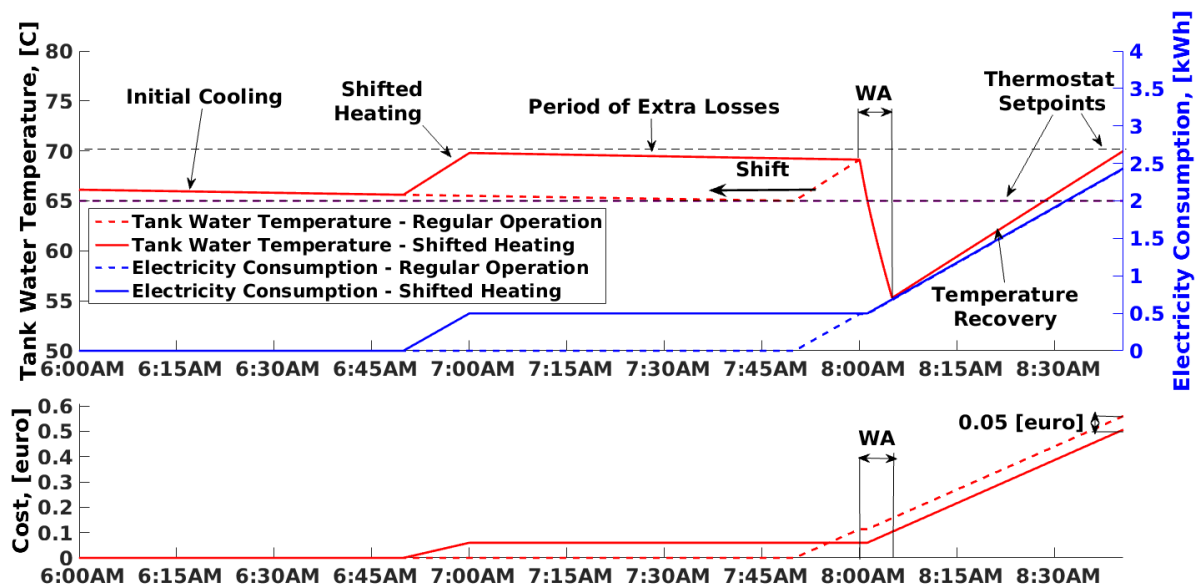

Figure 5.35: WH load shifting - Case of money savings.

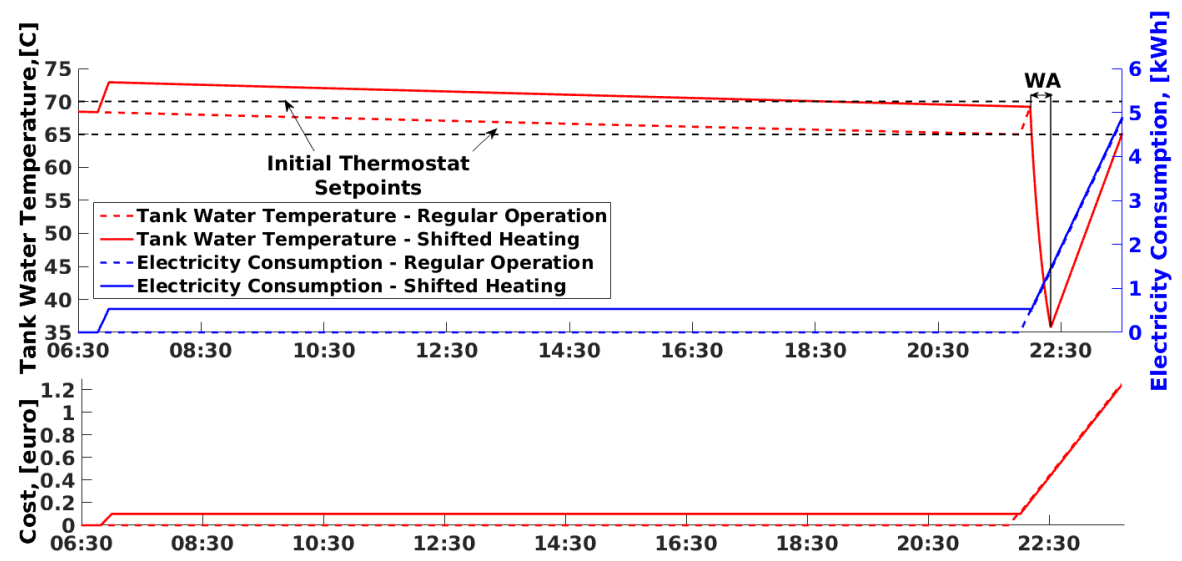

Figure 5.36: WH load shifting - No money savings.

i.e. by shifting the WH electricity consumption, one can achieve $€ 0.05$ of money savings as compared to the case without shifting.

In the previous example the WA happened at 8:00AM which was relatively close to the tariff price switch time at 7:00AM and the user comfort was fulfilled already by heating the water up to $69^{\circ} \mathrm{C}$. Consider now a scenario where the WA occurs at 10:00PM which is far beyond the moment of the price switch as shown in Figure 5.36. To force the WH to consume "cheap" electricity and at the same time to satisfy the user thermal comfort, the tank water should be heated up to $74^{\circ} \mathrm{C}$ before 7:00AM. However, such water heating increases the costs to $€ 1.25$ equal to the case without changing the WH setpoints.

We can conclude based on the above examples that the WH load shifting does not guarantee money savings if the user comfort is taken into consideration. 


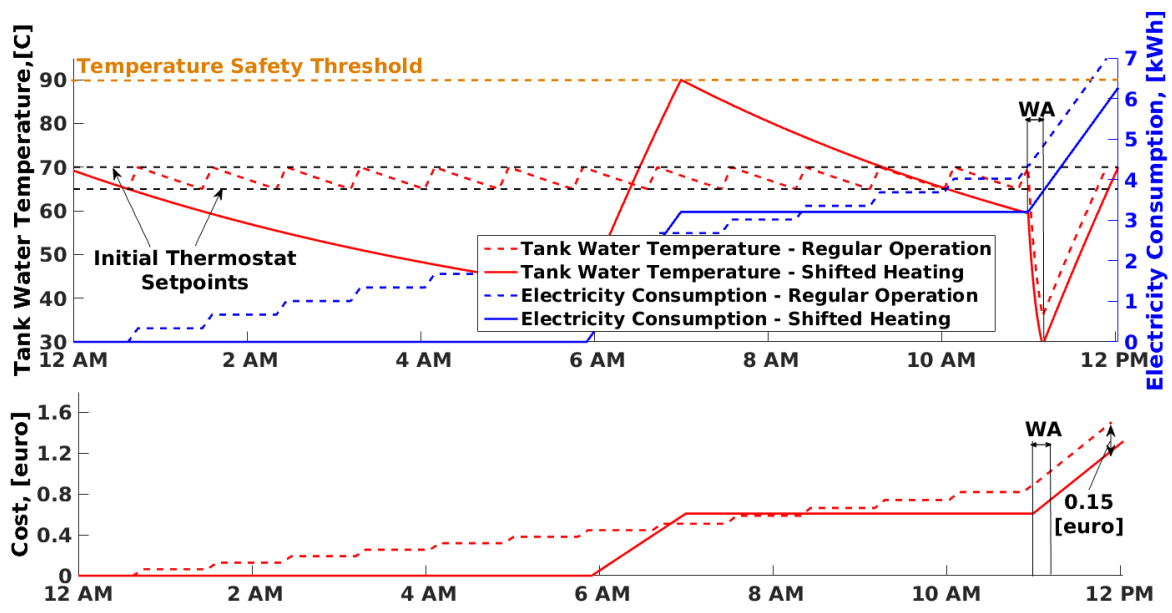

Figure 5.37: Negative impact on the user thermal comfort.

\section{EFfect of Load Shifting on User Comfort}

Besides the necessity to account for the effects of the WH load shifting on heating costs illustrated in the previous section, there is also a need to consider the implications of the load shifting on the end-user comfort.

Consider the following example where the technical parameters of the WH do not allow to efficiently shift the WH load comfort wise. In this example, the electricity tariff offers a reduced electricity rate between 12:00AM and 7:00AM. A WA occurs at 11:00AM and requires the tank water temperature to be at least $70^{\circ} \mathrm{C}$ at the beginning of the WA to meet the user comfort needs. Pursuing the money savings, we want to initiate the water heating prior to 7:00AM while providing the required user comfort. However, the water has to be heated to nearly the boiling point of $100^{\circ} \mathrm{C}$ in order to satisfy the user comfort which is not allowed by safety reasons. Heating the water up to only the maximum allowed $90^{\circ} \mathrm{C}$ will result in the tank water temperature value equal to only $60^{\circ} \mathrm{C}$ instead of the required $70^{\circ} \mathrm{C}$, which deteriorates the user thermal comfort. Thus, even though the user will gain money savings in that case, the WH load shifting will disrupt his thermal comfort.

Based on the considered example, we can conclude that shifting the WH electricity consumption might not be always acceptable due to the user comfort preferences.

To summarize, the simplified examples for the case of a single WA considered in Section 5.5.1 and Section 5.5.1 show that money savings due to the load shifting hinge upon a number of factors including:

- the tariff structure (times and prices);

- time- and comfort-related parameters of WAs;

- technical parameters of the WH such as the heat loss coefficient $U A$, the volume of the WH tank and heating capacity;

- the ambient temperature. 
The situation with an efficient WH load shifting with respect to both money savings and user comfort becomes even more entwined in case of multiple WAs. As discussed in Section 5.4.1, there is no straightforward way to efficiently schedule the heating periods without considering the influence of the solutions for preceding WAs on the following WAs.

Similar to the energy-comfort problem formulated in Section 5.4.1, the money-comfort problem can be formulated as follows.

\section{MONEY-COMFORT PROBLEM}

Given a WH with certain engineering characteristics and a daily profile of hot water activities with associated user thermal comfort preferences, find the best schedule of water heating periods and hot water flow rates that minimize the daily costs for water heating, while satisfying the required user comfort.

\subsubsection{Our Approach}

Our approach to the money-comfort problem in a scenario where multiple WAs can occur during a day is similar to the approach to the energy-comfort problem described in Section 5.4.2. In our approach, we aim to satisfy the required user thermal comfort by adopting the pre-heating control and the flow control introduced in Chapter 4 . That is to say, we remove the requirement for the fixed thermostat setpoints of the conventional deadband control. Instead, the water heating is performed to possibly higher temperatures (but not higher than the maximum safety allowed temperature) prior to WAs and in compliance with the user thermal comfort needs. We account for the user's intention to benefit financially by initiating the water heating periods preferably during the periods of low electricity prices, although it might be the case that water needs to be heated also during the periods of high prices to meet the user comfort requirements.

\subsubsection{Assumptions}

The assumptions for the money-comfort problem formulated in Section 5.5.1 resemble the assumptions for the energy-comfort problem in Section 5.4.3, i.e. we utilize Assumption 1 to Assumption 9.

An additional assumption for the money-comfort problem is the availability of the electricity prices for a day ahead.

\subsubsection{Price Model}

To solve the money-comfort problem, we modify the energy model developed in Section 5.4 and based on it introduce a new discrete-time price model. By analogy to the energy model, the price model utilizes the discrete intra-day and WA timescales described in Section 5.4.6 and Section 5.4.7.

In addition to the input parameters outlined in Section 5.4.4, input to the price model also comprises the information about the electricity tariff, i.e. the electricity prices and their times. Based on this input and assumptions in Section 5.5.3, the price model finds 
the best time intervals to switch on/off the WH on the intra-day timescale and the best tank water temperature values that minimize the daily heating costs while ensuring the required user thermal comfort. Firstly, the energy model deals with optimization of the heat injections into the $\mathrm{WH}$ and decides upon the state of the $\mathrm{WH}$, i.e. switched on or off, on every time interval of the discretized timescale. Secondly, the model finds the best tank water temperature values. These values (1) are associated with the found states of the heating elements and (2) carry the information about the best hot water flow rates implicitly found by the price model ${ }^{9}$. The model explicitly deals with two types of decision variables, namely the state of the WH (on/off) and the tank water temperature.

Akin the energy model, the output of the price model are two vectors $\mathbf{x}=\left\{x_{i} \mid x_{i} \in\right.$ $\{0,1\}, i \in[1, N]\}_{1 \times N}$ and $\mathbf{T}=\left\{T_{i} \mid T_{i} \in\left[T_{\text {cw }}, T_{\text {wh } \max }\right], i \in[1, N]\right\}_{1 \times N}$, where $N$ is the number of time intervals on the intra-day timescale. Based on the resulting values in $\mathbf{x}$ and $\mathrm{T}$ it is possible to restore the corresponding values of the hot water flow rates $\mathbf{m}=\left\{\dot{m}_{i} \mid \dot{m}_{i} \in\right.$ $\left.\left[0, \dot{m}_{\mathrm{d}, i}\right], i \in[1, N]\right\}_{1 \mathrm{x} N}$ by using Equation 2.2 .

To build the price model, we adopt and modify the energy model formalized in Equation 5.12 and Equation 5.5 to Equation 5.11. We argue that the constraints for the energy model sufficiently account for the thermodynamical model of the $\mathrm{WH}$, time-related and comfort-related parameters of the WAs during a day. There exist a need to only reformulate the objective function of the energy model in Equation 5.12 so that it captures the heating costs instead of the heat losses. The resulting price model represents a new (and separate) optimization model suitable for solving the money-comfort problem. The proposed price model is further described in detail.

\subsubsection{Formalization of Price Model}

Similar to the energy model, one has to accomplish the preparational steps described in Section 5.4.8 in order to obtain the matrices $\mathrm{A}^{+}, \mathrm{A}^{-}$and $\mathrm{D}^{+}, \mathrm{D}^{-}$. These matrices serve as input to the price model.

\section{Model Constraints}

A set of constraints for the price model is identical to the constraints for the energy model in Section 5.4.8, i.e. the constraints 5.5 to 5.11 are valid for the price model too.

\section{Objective Function}

Let $\left.\lambda=\left\{\lambda_{i} \mid 0<\lambda_{i} \leq \lambda_{\max }\right], i \in[1, N]\right\}_{1 \mathrm{x} N}$ be a vector of given electricity prices for a day ahead such that $\lambda_{i}$ represents an electricity price on the $i$-th time interval of the intra-day timescale $i \in[1, N]$.

To solve the money-comfort problem means to find the values of the elements in $\mathbf{x}$ and $\mathrm{T}$ that minimize the water heating costs while satisfying the user comfort. By multiplying the (unknown) elements of the vector of the WH states $\mathbf{x}$ by the corresponding (known) elements of vector of the electricity prices $\lambda$ and summing them up (or performing a vector multiplication), we obtain an expression that describes the daily costs for water heating:

$$
\lambda \mathbf{x}^{T}=\sum_{i=1}^{N} \lambda_{i} x_{i}
$$

\footnotetext{
${ }^{9}$ The model does not find the hot water flow rates explicitly, but rather decides upon the tank water temperature values with respect to the hot water flow rates.
} 
where $\lambda$ is a row vector of prices; $\mathbf{x}$ stands for a row vector of the WH states.

Since our goal is to minimize the daily water heating costs, we need to minimize the expresion in Equation 5.14. Therefore, the money-comfort problem can be formalized as follows:

$$
\min \left[\lambda \mathbf{x}^{T}\right]
$$

subject to the constraints in Equation 5.5 to Equation 5.11 specified in Section 5.4.8.

The problem in Equation 5.15 and Equation 5.5 to Equation 5.11 is a Linear Programming optimization problem.

By analogy to the energy model, the solution of the price model is two-fold. Firstly, the solution is represented by the binary vector $\mathbf{x}=\left\{x_{i} \mid x_{i} \in\{0,1\}, i \in[1, N]\right\}_{1 \times N}$ that defines on/off states of the WH on the intra-day timescale. Secondly, the price model returns a vector of the tank water temperatures $\mathrm{T}=\left\{T_{i} \mid T_{i} \in\left[T_{\mathrm{cw}}, T_{\mathrm{wh} \max }\right], i \in[1, N]\right\}_{1 \mathrm{x} N}$ that hold at the end of $N$ intervals, if the solutions in $\mathbf{x}$ are implemented.

The needed vector of the hot water flow rates $\dot{\mathbf{m}}=\left\{\dot{m}_{i} \mid \dot{m}_{i} \in\left[0, \dot{m}_{\mathrm{d}, i}\right], i \in[1, N]\right\}_{1 \mathrm{x} N}$, can be restored by substituting the found values in $\mathbf{x}$ and $\mathbf{T}$ into Equation 2.2 and solving it iteratively for $\dot{m}_{i}, i \in[1, N]$.

\subsubsection{Performance Evaluation}

In this section, we evaluate the performance of the proposed price model specified in Equation 5.15 subject to the constraints in Equation 5.5 to Equation 5.11 in reaching the money savings while satisfying the required user thermal comfort based on a series of simulations.

The simulation results were compared to the results of the deadband control during the regular operation of the $\mathrm{WH}$ as well as to the results of the energy model introduced in Section 5.4.

While obtaining the results for the price model and the energy model, we aimed at providing the user thermal comfort at the level comparable ${ }^{10}$ to the level of comfort delivered to the user during the deadband control.

We utilized a set of profiles containing the information about the time-related and comfort-related parameters of WAs identical to those used in simulations for the energy model in Section 5.4.9.

For the demonstrative purposes, we utilized a double-price tariff with $0.10[€ / \mathrm{kWh}]$ cost during the night time period (rolling between 23:00 and 06:30) 0.23 [€/kWh] during the day-time period in all the simulations.

We, firstly, ran the simulations for a period of a typical working and a typical weekend day chosen arbitrary. Taking into account that in the real life the user might choose in favor of the higher comfort level or the lower money expenses than in the deadband control, we have built Pareto fronts of solutions ranging from the most comfortable to the most cheap (money-wise) solutions.

We further extended the simulation period to 8 days sequential days D1-D8 and use a period of 7 days D2-D8 for checking the influence of daily hot water usage patterns on the

\footnotetext{
${ }^{10}$ As already mentioned in Section 5.4.9, a slight deviation in the comfort levels can be explained by by discretization of the intra-day timescale (in our case the granularity $\Delta t=5[\mathrm{~min}]$ ), the accuracy of the comfort constraints satisfaction, etc. A higher conformity of the comfort levels can be attained, in particular, by choosing a smaller granularity of the intra-day timescale.
} 


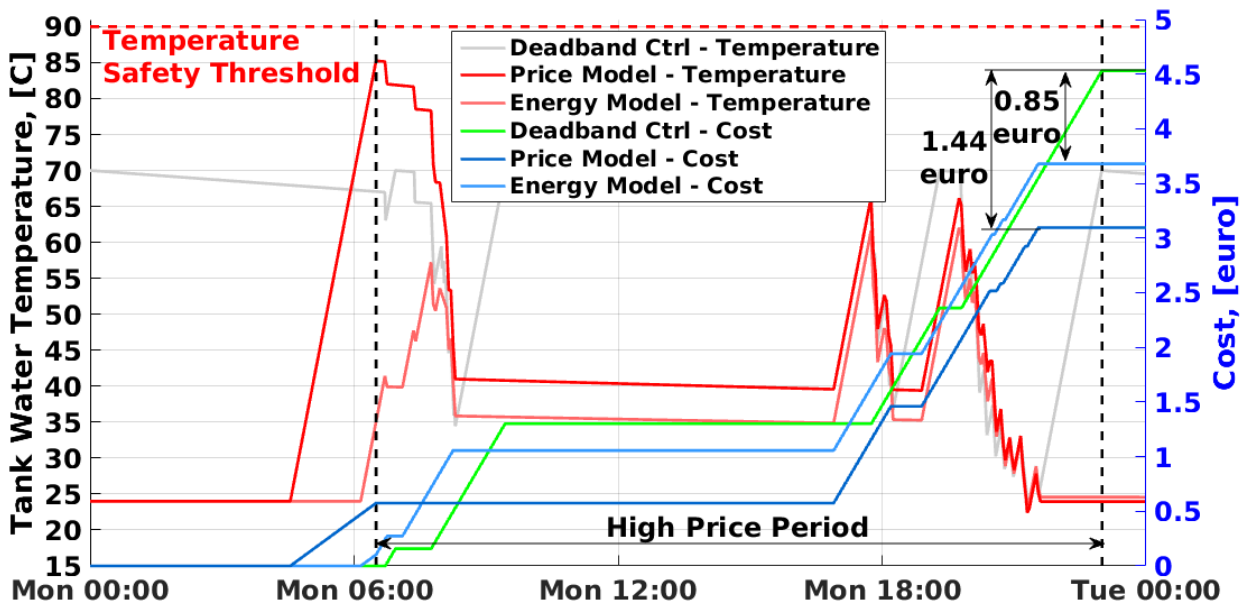

Figure 5.38: Typical working day - Money savings.

money savings. We thus do drop away the results for the day D1 in order to exclude the assumption for the initial temperature $T_{0}=15^{\circ} \mathrm{C}$ on the simulation results.

\section{Single Day Simulations}

The simulation results for a typical working day are shown in Figure 5.38 to Figure 5.41.

As Figure 5.38 shows, the solution of the price model turns to be $\approx 0.6[€]$ cheaper than that of the energy model under (almost) the same level of the user thermal comfort (Figure 5.41). Specifically, the price model responds to electricity price change at 06:30 by shifting around $6.1[\mathrm{kWh}]$ of the WH electricity consumption prior to the high-price period. The price model attempts to pre-store as much low cost heat as possible to minimize the daily heating costs. As it can be seen, the tank water temperature climbs up to a much higher value $\left(85^{\circ} \mathrm{C}\right)$ than in case of the energy model. In contrast to the energy model, the the price model aiming at minimization of costs keeps the WH shut off during the entire period of the morning peak of the hot water usage once the maximum temperature is established at 06:30. This justifies the need of water heating to such a high temperature.

Based on the plot of the electricity consumption in Figure 5.39, we can find that the electrical energy consumed by the price model during the morning period exceeds by 0.8 $[\mathrm{kWh}]$ the electricity consumption of the energy model, while the user comfort during this period is equal to 0 [unit] in both cases. Additionally, as a result of such an intense morning heating, the price model brings higher tank water temperature values during the afternoon as compared to the energy model, meaning the higher heat losses (see Figure 5.40). Nevertheless, the optimization solver has made a decision to spend 2 [kWh] extra electricity in the morning instead of the evening, because the netto cost in the morning-afternoon (defined as the cost of the electricity consumed in the morning minus the expenses for the heat losses during the afternoon) is lower than the cost of water heating during the evening period, as in case of the energy model. The total electricity savings achieved by 


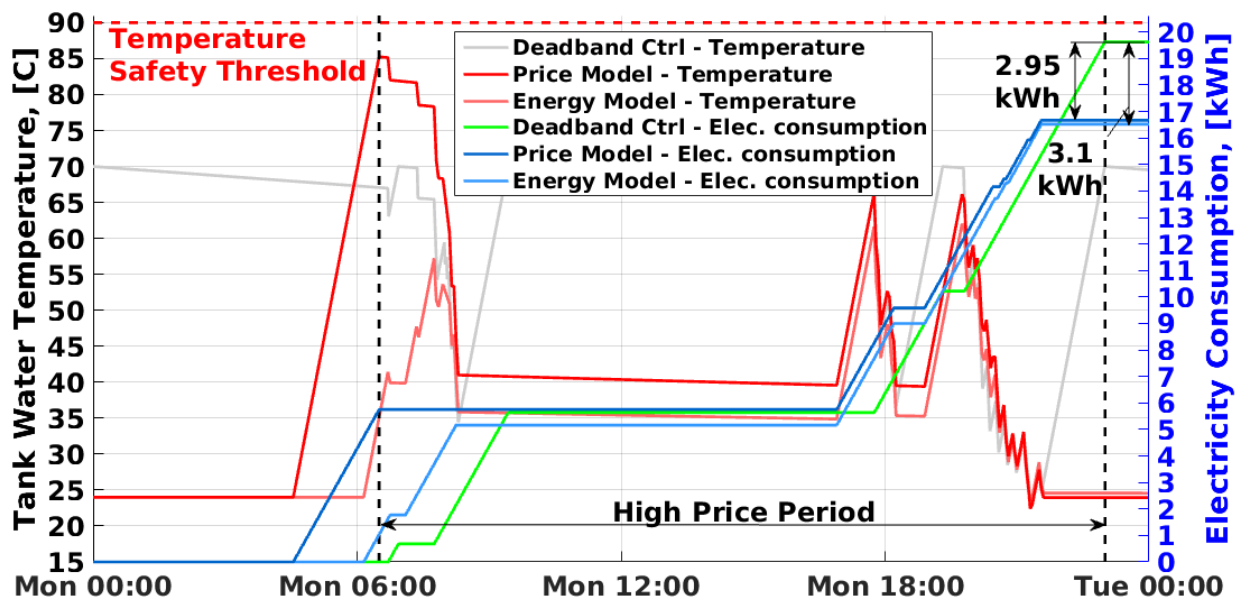

Figure 5.39: Typical working day - Electricity consumption.

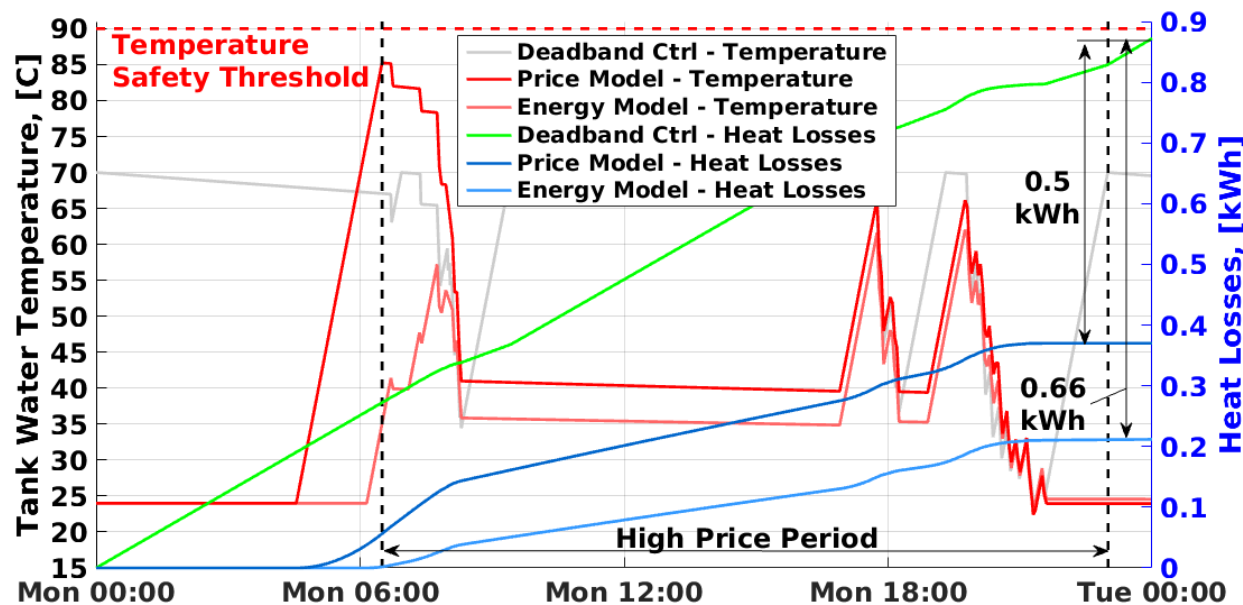

Figure 5.40: Typical working day - Heat losses.

the price model are 0.15 [kWh] smaller than those achieved by the energy model, which comes, in essence, from two different objective functions.

As a result of optimization tolerances and the rounding errors in our simulations, the thermal comfort provided by the price model is insignificantly higher (by 0.04 [unit]) than the thermal comfort delivered by the energy model, as shown in Figure 5.41.

The solutions for the selected working day and weekend day ranging from the most cheap money-wise to the most comfortable ones are represented on Pareto fronts in 


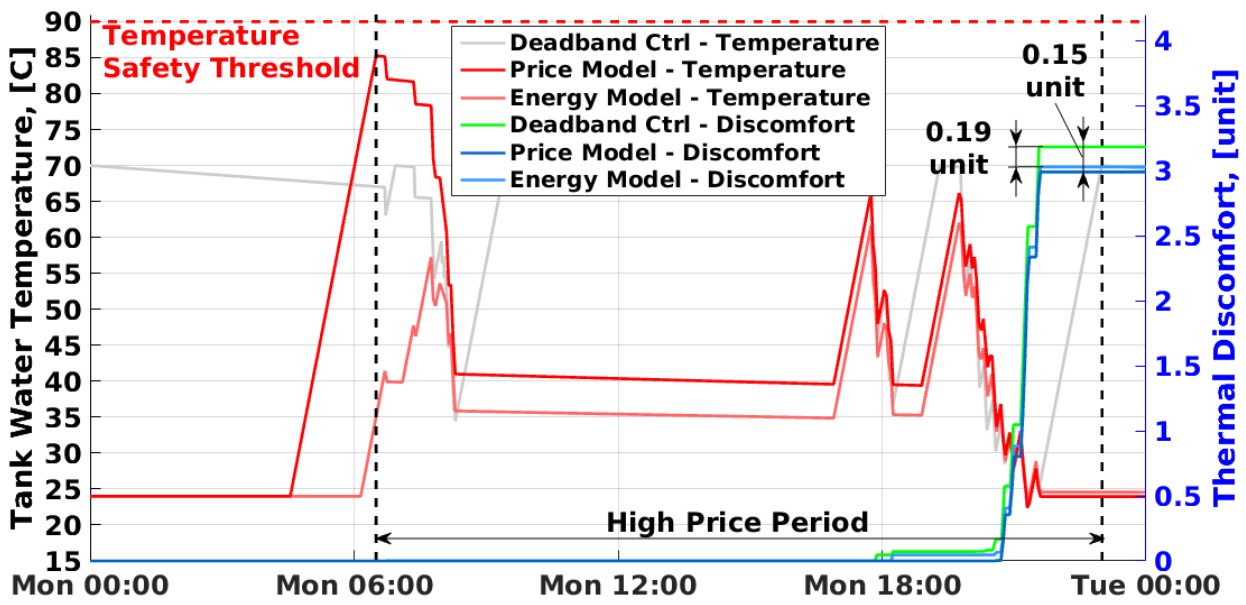

Figure 5.41: Typical working day - Thermal comfort.

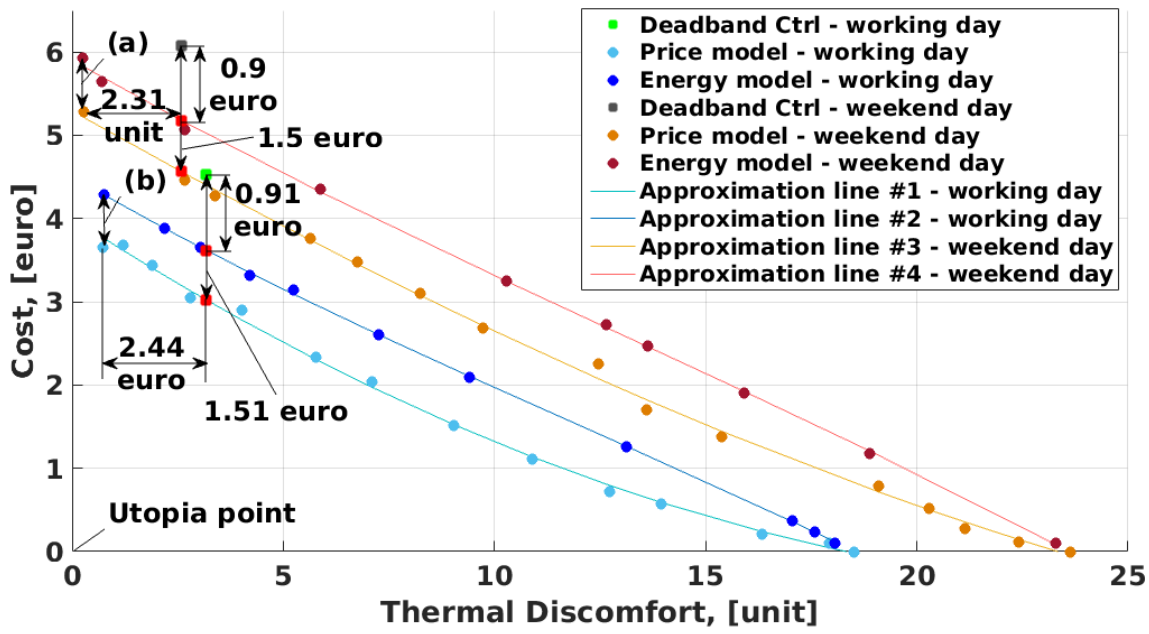

Figure 5.42: Pareto fronts - Typical working \& weekend days.

Figure 5.42.

As Figure 5.42 illustrates, the price model renders more price-effective solutions than the energy model and the deadband control both during the considered working and weekend days. Furthermore, the money savings are slightly higher during the working day, which can be clarified by the intensity and frequency of WAs during the weekend. That is to say, the price model is incapable to shift more electricity consumption to the low price period on the weekend day without the subsequent degradation of the user comfort. 
The price difference between the most comfortable solutions found by the price and energy models account for around $\approx 0.64[€]$ as referred by (a) and (b) in Figure 5.42.

\section{Simulation Results for Multiple Days}

We extended the simulation period of the price model to eight successive days D1-D8, while using the period of one week D2-D8 for estimation of its performance.

The simulation results are illustrated in Figure 5.43 to Figure 5.45.

The simulation results for D2-D8 are summarized in Table 5.5 and Table 5.6.

Table 5.5: Simulation results for one week D2-D8.

\begin{tabular}{|c|c|c|c|c|c|}
\hline $\begin{array}{c}\text { Control } \\
\text { Type }\end{array}$ & $\begin{array}{c}C, \\
{[€]}\end{array}$ & $\begin{array}{c}E_{\mathrm{e}}, \\
{[\mathrm{kWh}]}\end{array}$ & $\begin{array}{c}E_{\mathrm{d}}(\mathrm{b}) \\
{[\mathrm{kWh}]}\end{array}$ & $\begin{array}{c}Q_{\text {loss, }}, \\
{[\mathrm{kWh}]}\end{array}$ & $\begin{array}{c}D_{\mathrm{T}}, \\
{[\mathrm{unit}]}\end{array}$ \\
\hline $\begin{array}{c}\text { Deadband } \\
\text { Ctrl. }\end{array}$ & 36.79 & 159.79 & 262.59 & 5.89 & 27.48 \\
\hline $\begin{array}{c}\text { Energy } \\
\text { Model }^{(\mathrm{a})}\end{array}$ & 29.92 & 136.49 & 244.28 & 2.05 & 25.65 \\
\hline Price Model $^{(\mathrm{a})}$ & 28.2 & 138.75 & 245.71 & 2.39 & 23.98 \\
\hline
\end{tabular}

(a) corresponds to the control option (i) $T_{\mathrm{d}}(t)=T_{\text {c.min }}(t)$.

(b) The demanded energy $E_{\mathrm{d}}$ is present for a reference purpose only.

Note! The green color means improvement of a parameter as compared to other values in the same column.

The percentages of the corresponding changes of the electricity consumption, heat losses and user thermal comfort in comparison with the deadband control are listed in Table 5.6.

Table 5.6: Simulation results for one week D2-D8 - Percentages.

\begin{tabular}{|c|c|c|c|c|}
\hline Control Type & $\Delta C,[\%]$ & $E_{\mathrm{e}, \text { save }},[\%]$ & $\Delta Q_{\text {loss }},[\%]$ & $\Delta D_{\mathrm{T}},[\%]$ \\
\hline Energy Model & 18.67 & 14.58 & 65.20 & 6.66 \\
\hline Price Model & 23.35 & 13.17 & 59.42 & 12.74 \\
\hline
\end{tabular}




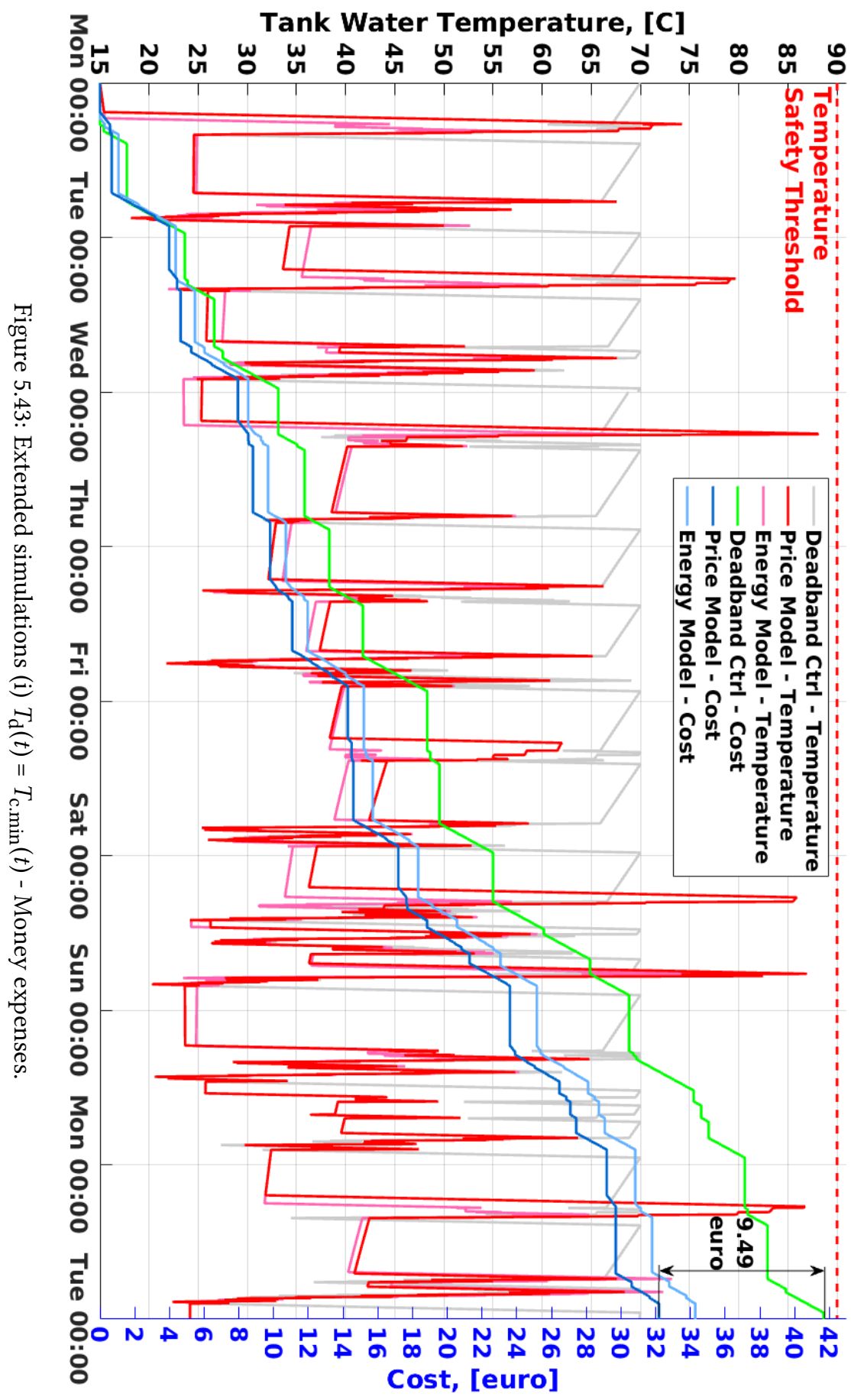




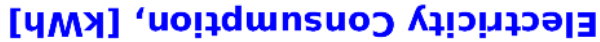

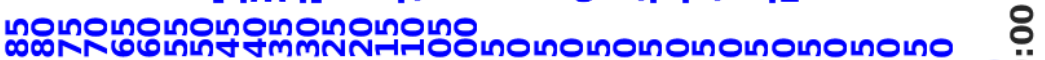

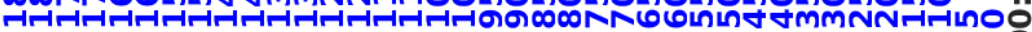

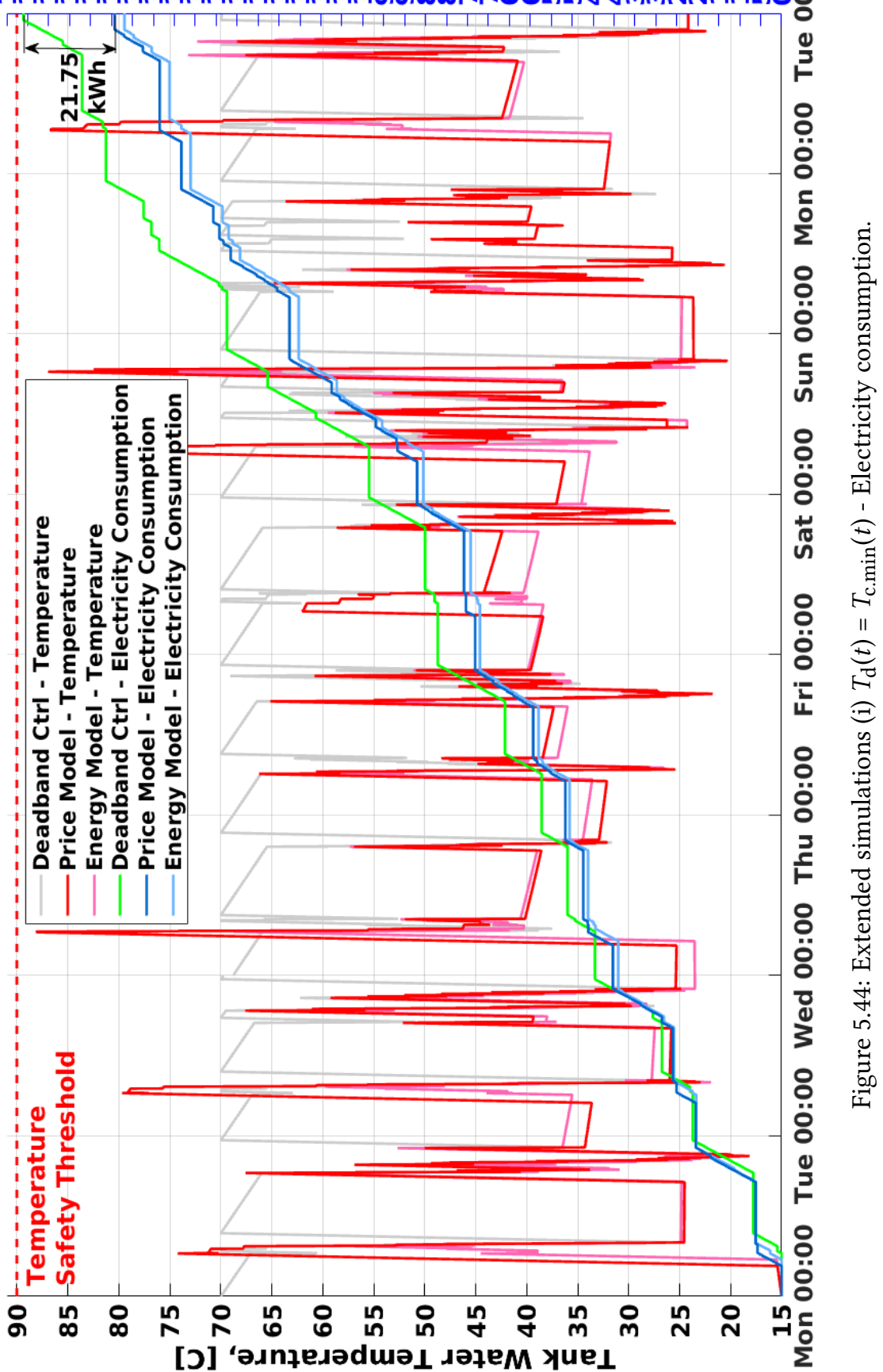




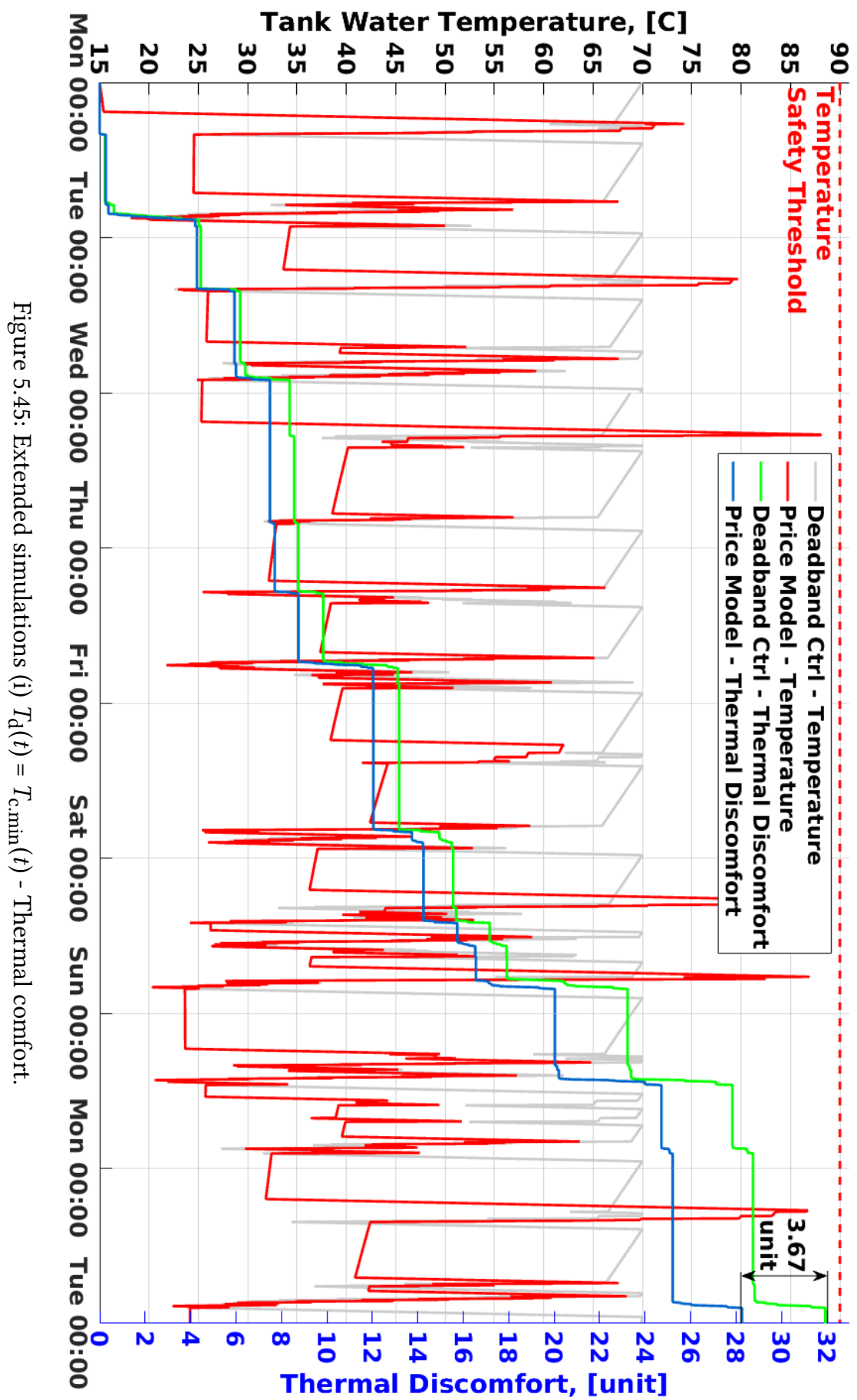


Table 5.7: Energy Model - Daily averages.

\begin{tabular}{|c|c|c|c|c|c|c|c|c|}
\hline \multirow{3}{*}{ Day Type } & \multicolumn{9}{|c|}{ Price Model } \\
\cline { 2 - 9 } & \multicolumn{2}{|c|}{$\tilde{C}_{\text {save }}$} & \multicolumn{2}{c|}{$\tilde{E}_{\text {e, save }}$} & \multicolumn{2}{c|}{$\Delta \tilde{Q}_{\text {loss }}$} & \multicolumn{2}{c|}{$\Delta \tilde{D}_{\mathrm{T}}$} \\
\cline { 2 - 9 } & $€$ & $\%$ & $\mathrm{kWh}$ & $\%$ & $\mathrm{kWh}$ & $\%$ & unit & $\%$ \\
\hline working & 0.98 & 22.09 & 3.21 & 15.98 & 0.56 & 64.91 & 0.09 & 12.19 \\
\hline weekend & 0.99 & 13.82 & 3.64 & 11.52 & 0.68 & 65.79 & 0.51 & 10.02 \\
\hline
\end{tabular}

Table 5.8: Price Model - Daily averages.

\begin{tabular}{|c|c|c|c|c|c|c|c|c|}
\hline \multirow{3}{*}{ Day Type } & \multicolumn{9}{|c|}{ Price Model } \\
\cline { 2 - 9 } & \multicolumn{2}{|c|}{$\tilde{C}_{\text {save }}$} & \multicolumn{2}{c|}{$\tilde{E}_{\text {e, save }}$} & \multicolumn{2}{c|}{$\Delta \tilde{Q}_{\text {loss }}$} & \multicolumn{2}{c|}{$\Delta \tilde{D}_{\mathrm{T}}$} \\
\cline { 2 - 9 } & $€$ & $\%$ & $\mathrm{kWh}$ & $\%$ & $\mathrm{kWh}$ & $\%$ & unit & $\%$ \\
\hline working & 1.22 & 28.21 & 2.9 & 14.75 & 0.51 & 58.31 & 0.26 & 15.15 \\
\hline weekend & 1.25 & 17.15 & 3.27 & 10.43 & 0.49 & 62.32 & 1.11 & 15.39 \\
\hline
\end{tabular}

The price model demonstrates clear spikes of the tank water temperature before the start of the high-price period at 06:30 across all the simulated days D1-D8. As a result of shifting of the WH electricity consumption to the low-price periods, the price model is capable to achieve by $4.68 \%$ higher money savings than the energy model during the same period period D2-D8. On the hand, the electricity savings achieved by the price model are by $1.41 \%$ lower than those gained by the energy model, as demonstrate Table 5.5 and Table 5.6. This difference between the money savings and electricity savings points to the fact that shifting of the water heating to the low-price periods can result in the increased electricity consumption. In other words, the heat pre-stored far upfront its actual utilization causes additional heat losses in the price model which lead to higher electricity usage, although this turns to be a less expensive solution under the considered electricity tariff and hot water usage profiles.

Finally, based on the considered period D2-D8, we obtained the daily averages expected from the energy and price models, shown in Table 5.7 and Table 5.8.

\subsection{Conclusion}

In this chapter, we tackled limitations of the conventional deadband control of tank electric water heaters (WHs), considering a scenario where multiple hot water activities (WAs) can occur during a day. Specifically, we focused on the following downsides of the deadband control:

1. inefficient user comfort management;

2. energy inefficiency originating from stand-by heat losses;

3. incapability to shift the electricity consumption of the $\mathrm{WH}$ in response to variable electricity prices.

To overcome the above limitations of the deadband control, we proposed a new control mechanism for a domestic hot water system. The control mechanism employs two optimization models, namely the energy model and the price model, each addressing distinct problems of the deadband control. 
More specifically, the energy model allows for minimization of the daily electricity consumption of the WH while satisfying the user thermal comfort needs, therefore addressing Problem 1 and Problem 2. The energy model achieves the electricity savings by (a) minimizing the heat losses, and (b) exploiting the user thermal tolerance, i.e. the model provides the demanded thermal energy at the minimum, yet comfortable, level.

Given a variable electricity price tariff, the price model aims to minimize the daily water heating costs taking into account the user-desired thermal comfort during WAs. The price model thus addresses Problem 1 and Problem 3.

The two tailored models pursue different optimization objectives and thus should be used separately from each other. However, since the price model was built upon the energy model, the two models can be combined into a single optimization model, for example, by representing their objective functions by a single weighted sum as follows:

$$
\min \left[\varphi \mathbf{1} \mathbf{T}^{T}+\psi \boldsymbol{\lambda} \mathbf{x}^{T}\right]
$$

where 1 is a row vector of ones, $\varphi \geq 0$ and $\psi \geq 0$ stand for the weight coefficients that signify the importance of satisfaction of the first term (minimization of the electricity consumption) and the second term (minimization of the heating costs), respectively. Thus, the objective function in Equation 5.16 unites both the energy and price models. In our case, the pure energy model corresponds to $\varphi=1$ and $\psi=0$, while the pure price model refers to $\varphi=0$ and $\psi=1$.

In this chapter, we evaluated the performance of the devised energy and comfort models separately based on simulations. The simulation results were compared to the results of the deadband control. Since the results of the models much depend on the presence of the tap water temperature stabilization during WAs, we have considered two options for the tap water temperature provision, i.e. with and without the user requirement for a stable temperature.

The simulation results for the energy model showed that in the scenario without a temperature stabilization the model was capable to achieve in average $2.10 \%$ and $0.72 \%$ electricity savings during the working and weekend days, respectively, as compared to the deadband control. In a scenario with a temperature stabilization, the energy model supplied a nearly stable tap water temperature during WAs on the lowest user-comfortable level and achieved $15.98 \%$ and $11.52 \%$ electricity savings in average during the working and weekend days, respectively. These values can vary depending on the degree of the given user thermal tolerance.

The price model in a scenario with a double-price ("day-night") tariff and with the tap water temperature stabilization demonstrated $28.21 \%$ and $17.15 \%$ money savings in average during working and a weekend days, respectively. 


\section{6}

\section{CONCLUSION}

C urrent trends of electricity production can be characterized by a strong contribution of renewable energy sources (RES) represented by photovoltaic panels, wind mills, biogas generators, etc. on different scales of the European energy system.

One of the problems that comes along with the emergence and distribution of RES is the uncertainty of their power generation capacities as these are highly dependent on meteorological conditions. Furthermore, the variability of the electricity generation today can be also induced by small consumers with on-site generation (e.g., rooftop solar panels). Today, such "prosumers" are allowed to sell their generated energy excesses (e.g., feed-in tariffs) and increase the system's generation capacities. However, the amount of electrical energy they can offer varies depending on the amount of electricity they decide to use themselves, so-called self-consumption.

High variability of the electricity production hampers planning of the generation and consumption portfolios of balance responsible parties (BRPs) on the day-ahead and intraday electricity markets. Uncertainties in the power supply forecasts may negatively impact the nominations and lead to a real-time energy imbalance for BRPs. System operators have to timely restore the system balance by activating reserves (e.g. frequency restoration) and balancing (e.g., upward regulation) in order to prevent major imbalances and power outages. While in the past the role of RES in total electricity generation was less significant, utility companies presently encounter difficulties in acquiring reliable forecasts of energy production of RES, which presents risks for the stability of the system.

Aside from RES, the growing electricity consumption in the EU, which is partially caused by a widespread adoption of electrical vehicles, also calls for special attention. These groups of mobile loads may create unpredictable peaks of the power demand and may deteriorate the stability of the grid, if not properly coordinated.

In response to the problems brought by current trends in the electricity productiondemand chain, solutions to increase the energy efficiency on all the levels of the energy system are actively promoted. Demand response (DR) is commonly viewed as a perspective approach to deal with the problems that face the energy community today. While consumers provide some flexibility to modify their energy usage patterns, utility companies are interested in utilizing this flexibility in the best way to increase reliability and security 
of the electricity supply. The term "flexibility" used in this thesis relates to an ability of a consumer to reduce (or to shift) energy consumption during a specific period (to another period) that might be advantageous both for the consumer and for his utility company.

Despite the numerous benefits resulting from DR (e.g., reduction of monthly electricity bill under variable price tariffs), there is still a significant level of consumer resistance to DR adoption. The recent energy reports show that consumer concerns about the real possibility to benefit financially from DR by sacrificing own flexibility and possibly comfort, as well as the fear to loose control of their home appliances (e.g., as in case of utility-driven load management) impede a wide-scale residential consumer uptake of DR.

This thesis specifically concentrates on the problem of consumer comfort degradation in DR of residential loads. We tackle the problem on the level of a single household and a single household load, taking an electric tank water heater as a popular example of residential flexible loads in DR. Thus, provision of the user-desired comfort during hot water usage is identified in this thesis as a main enabler of consumer uptake of DR solutions for WHs. In some contexts, the objectives of consumers and utility companies can be conflicting. For example, an objective of a utility company to shave the peak demand by shutting down a group of residential WHs may be treated by consumers as an attempt to interfere with their comfort. However, in case the disconnection of WHs is executed cautiously with respect to the comfort of residents, consumers may be willing to participate in such load management.

In this thesis, we explore the ways to increase (or at least preserve) the end-user comfort during DR of a residential WH. Based on the review of the state-of-the-art solutions for DR of WHs in Chapter 2, we emphasize the necessity to account for the heat losses in control of the WH in order to achieve its energy efficient operation. At the same time, we illuminate the limitations of the existing approaches for user comfort modeling found in the literature and stress the need for a better representation of the user comfort during the hot water service.

In Chapter 3, we deal with the question of how to represent numerically human comfort during the hot water service. There is already a stack of well-established comfort models for space heating loads used by engineers when designing the residential space heating and cooling systems. By analogy to space heating, one can hypothesize about possibilities to put a number on user comfort in case of water heating loads. We demonstrate the possibilities to model the user comfort during the hot water usage and develop two user comfort models, namely the user thermal and flow comfort models. While the thermal comfort model can quantify the end-user discomfort resulting from the uncomfortable tap water temperature, the flow comfort model can represent numerically user dissatisfaction with the inconvenient tap water flow rate.

Chapter 4 investigates possible strategies to preserve/improve the user comfort during a single hot water activity (WA). Since WHs represent in essence storages for thermal energy, extra heat can be pre-stored beforehand an intense hot water usage that requires a lot of hot water (or smaller amounts of very hot water) so that the hot water is available during the entire hot-water event and the thermal comfort of the user is maintained at the required level. Another strategy found, is the regulation of the hot water outflow from the WH. By gradually decreasing the amount of hot water leaving the tank, it is possible to prolong the availability of hot water in the tank. Conversely, in scenarios where the 
user desires a stable tap water temperature the hot water outflow from the water tank can be increased in progress of the performed hot water activity. These pre-heating and flow control strategies represent the atomic measures for improvement of the user comfort by means of control. The strategies act as building blocks in a more sophisticated control mechanisms for multiple WAs introduced in Chapter 5.

The problems solved in Chapter 5 refer to reconciliation of (sometimes) conflicting consumer objectives such as (i) minimization of the daily electricity consumption for water heating and minimization of user thermal comfort disruptions, (ii) minimization of the daily water heating costs and minimization of the user thermal discomfort. More specifically, we aim at simultaneous satisfaction of the above functions by means of a multiobjective optimization. The chapter starts with a control mechanism for balancing the daily electricity consumption for water heating and user thermal comfort, i.e. the energy model. The goal of minimization of the electricity consumption is approached by minimizing the average tank water temperature on a daily timescale. By doing such a translation, we attempt to reduce the $\mathrm{WH}$ operational temperatures during the day and thus also take care about the heat losses. We obtain a Pareto front of non-dominated solutions for the energy model by first solving each of the two objectives (minimize energy and minimize thermal discomfort) independently from each other, which gives us a range of possible user thermal comfort values. After that, we minimize the average daily tank water temperature for each of the user (dis)comfort values from the found range, which constitutes the solution of our multi-objective optimization problem. To evaluate the proposed control algorithm, we compare its results with the results of the conventional deadband control of the WH under the same level of user comfort. The simulation results show that consumer can attain:

- an average electricity savings of $2.10 \%$ during the working days and $0.72 \%$ during the weekend days in case of no user thermal tolerance;

- an average electricity savings of $15.98 \%$ during the working days and $11.52 \%$ during the weekend days when the user exhibits some thermal tolerance,

as compared to the conventional deadband control under the same levels of the user thermal comfort.

Next to the energy model, we propose a model for simultaneous minimization of daily water heating costs and user thermal discomfort in the price-based DR of the $\mathrm{WH}$, i.e. the price model. This mechanism allows for shifting the WH load to the periods of low electricity rates, while constraining the amount of the shifted load by the user-desired comfort level. Unlike the energy model, the devised price model directly minimizes the electricity costs for activation of the WH heating elements on each time interval during the day. As in case of the energy model, the results obtained by the price model are compared with the results of the deadband control under the double-price tariff and the same level. The simulation results of the price model demonstrate that a consumer can achieve the average money savings of $28.21 \%$ during the working days and $17.15 \%$ during the weekend days in case of the present user thermal tolerance in comparison with the deadband control under the same levels of the user thermal comfort.

Summarizing, the distinguishing features of the proposed control mechanism represented by the energy and price models are as follows: 
- deterministic linear programming optimization approach;

- in general, the minimization of the user thermal discomfort is represented as a separate objective function; however, to provide the desired and known level of the user thermal comfort, the user comfort can be instead imposed as constraints for the optimization problems;

- allows to exploit the thermal tolerance offered by the users;

- accounts for the presence of mixer taps, and user thermal comfort is modeled through the tap water temperature variable;

- suitable for residential hot water systems with more than one tapping point;

- activity-based approach for comfort modeling where each WA can have its own user comfort requirements (time-varying user comfort);

- built upon the well-mixed WH tank model;

- the results of the optimization are (i) the state vector of the heating elements of the $\mathrm{WH}$ and (ii) the tank water temperatures to be established by control; hence the mechanism allows for the control of the heating elements or alternatively for the thermostat setpoint temperature control;

- hot water outflow from the WH tank is a control variable.

Based on the above discussion of the work done, we can answer the research questions raised in Chapter 1:

- How to represent numerically user satisfaction with the hot water service at home? Since the users feel and appreciate the tap water temperature and the tap water flow rate during hot water activities, it is meaningful to include these environmental variables in the user comfort model. Besides, different people can perceive the same water temperature differently, which refers to the need to consider personal variables in the user comfort model such as, for example, user-specific thermal tolerance. This personal variable can vary per user and per hot water activity performed. Furthermore, it is known that the time during which an individual experiences a comfort violation can have different impact on the user. The time aspect of the user comfort can be accounted for by integrating the instantaneous deviations of the controlled variable (e.g., the tap water temperature). A combination of these parameters can serve as input to the user comfort model.

- What is the optimization potential of WHs with regard to energy and money savings? The optimization potential of a given WH to save electrical energy and money is not only determined by its technical parameters such as volume, capacity and thermal conductivity of the WH tank and the tariff structure, but also by the impact on the end-user comfort. The latter is represented by the timing of the WAs during a day, desired user thermal comfort and the existance of the user thermal tolerance to colder temperatures. In general, interrelations of these parameters determine the optimization potential of the WH in terms of energy and money savings. 
- Is it possible to increase the energy efficiency of the WH operation without a decrease of the end-user comfort by means of control? Based on the simulation results of the proposed energy model, it is possible to reduce electricity consumption for water heating without deterioration of the user comfort as compared to the conventional deadband control. The extent of the energy savings is determined by technical parameters of the WH (e.g., insulation), operating conditions (e.g., the ambient temperature) and user comfort preferences.

- Can the price-based DR of WHs be implemented without deterioration of the user comfort? Based on the simulations of the proposed price model, the answer is positive. The amount of money savings depends on technical parameters of the $\mathrm{WH}$, operating conditions (e.g., the temperature of cold water), user comfort preferences and electricity rates. 



\section{BIBLIOGRAPHY}

\section{REFERE NCES}

[1] Jin-Ho Kim and Anastasia Shcherbakova. Common failures of demand response. Energy, 36(2):873-880, 2011.

[2] Eto Joseph. The past, present, and future of us utility demand-side management programs. Environmental Energy Technologies Division Ernest Orlando Lawrence Berkeley National Laboratory, 1996.

[3] Anna Magdalena Kosek, Giuseppe Tommaso Costanzo, Henrik W Bindner, and Oliver Gehrke. An overview of demand side management control schemes for buildings in smart grids. In 2013 IEEE international conference on smart energy grid engineering (SEGE), pages 1-9. IEEE, 2013.

[4] Energy Efficiency Directive. Directive 2012/27/eu of the european parliament and of the council of 25 october 2012 on energy efficiency, amending directives 2009/125/ec and 2010/30/eu and repealing directives 2004/8/ec and 2006/32. Official fournal, L, 315:1-56, 2012.

[5] Q Qdr. Benefits of demand response in electricity markets and recommendations for achieving them. U.S. department of energy, 2006.

[6] European Commission. Demand response status in eu member states. Jrc scientific and policy report., 2016.

[7] A. Gutirrez E. Matallanas, M. Castillo-Cagigal et al. Neural network controller for active demand-side. Applied Energy, 91:90-97, 2012.

[8] V Giordano, Gangale F, Fulli G, and M Sanchez Jimenez. Smart grid projects in europe: Lessons learned and current developments. european commission, joint research centre. European Commission. Foint Research Center. Institute for Energy. The Netherlands, 2011.

[9] E.F. El-Saadany M.H. Albadi. A summary of demand response in electricity markets. Electric Power Systems Research, 78:1989-1996, 2008.

[10] Accenture. Understanding consumer preferences in energy efficiency in the utilities industry. https : / / WwW . accenture. com, 2010. Last visit:05/07/2020.

[11] Joseph Seymour and Terry Horsley. The seven types of power problems. APC, USA, 2005.

[12] TenneT Holding B.V. Empowering society. integrated annual report 2016. Technical report, 2016. 
[13] Alliander N.V. Consolidated annual results 2016 of alliander n.v. Technical report, 2016.

[14] Oddyssee-Mure project. Households energy data. https : / / WWW . indicators . odyssee-mure.eu/energy-efficiency-database.html. Last visit:28/03/2020.

[15] European Statistical Office. Energy consumption in households. Statistics report, 2019.

[16] Heat Roadmap Europe Project. Profile of heating and cooling demand in 2015. Deliverable, 2017.

[17] S. Werner U. Persson. Quantifying the heating and cooling demand in europe. Technical report, 2015.

[18] European Commission. Heat and cooling demand and market perspective. Jrc scientific and policy report., 2012.

[19] VHK for the European Commission. Water heaters - ecodesign and energy labels. market analysis. Draft final report., 2019.

[20] Maria Alejandra Zuñiga Alvarez, Kodjo Agbossou, Alben Cardenas, Sousso Kelouwani, and Loïc Boulon. Demand response strategy applied to residential electric water heaters using dynamic programming and k-means clustering. IEEE Transactions on Sustainable Energy, 11(1):524-533, 2019.

[21] Khalid Elgazzar, Howard Li, and Liuchen Chang. A centralized fuzzy controller for aggregated control of domestic water heaters. In Electrical and Computer Engineering, 2009. CCECE'09. Canadian Conference on, pages 1141-1146. IEEE, 2009.

[22] H Salehfar, PJ Noll, BJ LaMeres, MH Nehrir, and V Gerez. Fuzzy logic-based direct load control of residential electric water heaters and air conditioners recognizing customer preferences in a deregulated environment. In Power Engineering Society Summer Meeting, 1999. IEEE, volume 2, pages 1055-1060. IEEE, 1999.

[23] Jingshuang Shen, Chuanwen Jiang, and Bosong Li. Controllable load management approaches in smart grids. Energies, 8(10):11187-11202, 2015.

[24] Duke Energy. Energywise direct load control. https : / / www . duke-energy . com/home/products/energywise-home, 2017. Last visit:05/07/2020.

[25] International Renewable Energy Agency. Time-of-use tariffs. Innovation landscape brief, 2019.

[26] EC Smart Grid Task Force.European Commission. Task force for smart grids, vision and work programme. https://ec.europa.eu/energy/en/ topics/markets-and-consumers/smart-grids-and-meters/ smart-grids-task-force. Last visit:05/07/2020. 
[27] European Commission. Smart grids: From innovation to deployment', communication from the commission to the european parliament. http: / / eur- lex . europa . eu/LexUriServ/LexUriServ . do?uri=COM : 2011 : 0202 : FIN : EN : PDF, 2011. Last visit:05/07/2020.

[28] SETIS Magazine. Demand response - empowering the european consumer. https://setis.ec.europa.eu/ publications/setis-magazine/smart-grids/ demand-response-empowering-european-consumer, March 2014. Last visit:05/07/2020.

[29] Jasmin et al. Geppert. Preparatory study on smart appliances task 3 users.

[30] Wilma Mert, Jürgen Suschek-Berger, and Wibke Tritthart. Consumer acceptance of smart appliances. d 5.5 of wp 5 report from smart-a project.

[31] Quantum Consulting Sharon Mecum. A wish list for residential direct load control customers. Panels of the 2002 ACEEE Summer Study on Energy Efficiency in Buildings, 2002.

[32] S Hari Charan Cherukuri and B Saravanan. A novel dsm strategy for micro grids consisting of higher penetration of water heater loads. In 2018 National Power Engineering Conference (NPEC), pages 1-6. IEEE, 2018.

[33] Ali Haider, Will Stark, and Ted KA Brekken. Electric hot water heater primary frequency control. In 2018 IEEE Energy Conversion Congress and Exposition (ECCE), pages 2670-2675. IEEE, 2018.

[34] Sara Barja-Martinez, Pol Olivella-Rosell, Pau Lloret-Gallego, Roberto Villafafila-Robles, Andreas Sumper, Stig Ødegaard Ottesen, and Hoang Minh Tran. A scheduling optimization model of electric water heaters for electricity cost minimization with limited information. In 2019 8th International Conference on Modern Power Systems (MPS), pages 1-6. IEEE, 2019.

[35] Mei Wu, Yu-Qing Bao, Jinlong Zhang, and Tongzhou Ji. Multi-objective optimization for electric water heater using mixed integer linear programming. fournal of Modern Power Systems and Clean Energy, 7(5):1256-1266, 2019.

[36] Jidong Wang, Chenghao Li, Peng Li, Yanbo Che, Yue Zhou, and Yinqi Li. Mpcbased interval number optimization for electric water heater scheduling in uncertain environments. Frontiers in Energy, pages 1-15, 2019.

[37] Christophe Patyn and Geert Deconinck. Electric water heater control through informed fitted q-iteration. In 2019 IEEE PES Innovative Smart Grid Technologies Europe (ISGT-Europe), pages 1-5. IEEE.

[38] Qian Wu, Lingfeng Wang, and Bin Li. An optimized demand response strategy for electric water heaters and the associated impact on power system operational reliability. In 2017 International Smart Cities Conference (ISC2), pages 1-6. IEEE, 2017. 
[39] Peter Kepplinger. Autonomous demand side management of domestic hot water heaters. $\mathrm{PhD}$ thesis, Dissertation, University of Innsbruck, Innsbruck, Austria, 2015.

[40] Jamie Bartram. Legionella and the prevention of legionellosis. World Health Organization, 2007.

[41] Md Salman Nazir, Francisco D Galiana, and Alexandre Prieur. Unit commitment incorporating histogram control of electric loads with energy storage. IEEE Transactions on Power Systems, 31(4):2857-2866, 2015.

[42] David W Viola. Water temperature control and limitation. Plumbing Manufacturers Institute, 6, 2002.

[43] Marcel Roux, Mark Apperley, and MJ Booysen. Comfort, peak load and energy: Centralised control of water heaters for demand-driven prioritisation. Energy for Sustainable Development, 44:78-86, 2018.

[44] Bishnu P Bhattarai, Iker Diaz De Zerio Mendaza, Birgitte Bak-Jensen, Jayakrishnan R Pillai, Nava R Karki, Jake P Gentle, and Kurt S Myers. Active control of thermostatic loads for economic and technical support to distribution grids. In 2016 IEEE Power and Energy Society General Meeting (PESGM), pages 1-5. IEEE, 2016.

[45] M Hashem Nehrir, Runmin Jia, Donald A Pierre, and Donald J Hammerstrom. Power management of aggregate electric water heater loads by voltage control. In Power Engineering Society General Meeting, 2007. IEEE, pages 1-6. IEEE, 2007.

[46] Khalid Al-Jabery, Zhezhao Xu, Wenjian Yu, Donald C Wunsch, Jinjun Xiong, and Yiyu Shi. Demand-side management of domestic electric water heaters using approximate dynamic programming. IEEE Transactions on Computer-Aided Design of Integrated Circuits and Systems, 36(5):775-788, 2016.

[47] Koon Wong and Michael Negnevitsky. Optimisation of switching programs for demand side management of domestic hot water load. In 2013 Australasian Universities Power Engineering Conference (AUPEC), pages 1-6. IEEE, 2013.

[48] Ndumiso G Dlamini and Fabien Cromieres. Implementing peak load reduction algorithms for household electrical appliances. Energy Policy, 44:280-290, 2012.

[49] Ziming Zhu, Jie Tang, Sangarapillai Lambotharan, Woon Hau Chin, and Zhong Fan. An integer linear programming based optimization for home demand-side management in smart grid. In Innovative Smart Grid Technologies (ISGT), 2012 IEEE PES, pages 1-5. IEEE, 2012.

[50] Yan Chen, Ren Ping Liu, Chen Wang, Martin De Groot, and Zhiyuan Zeng. Consumer operational comfort level based power demand management in the smart grid. In 2012 3rd IEEE PES Innovative Smart Grid Technologies Europe (ISGT Europe), pages 1-6. IEEE, 2012. 
[51] Junji Kondoh, Ning Lu, and Donald J Hammerstrom. An evaluation of the water heater load potential for providing regulation service. In Power and Energy Society General Meeting, 2011 IEEE, pages 1-8. IEEE, 2011.

[52] Arnaldo Sepulveda, Liam Paull, Walid G Morsi, Howard Li, CP Diduch, and Liuchen Chang. A novel demand side management program using water heaters and particle swarm optimization. In Electric Power and Energy Conference (EPEC), 2010 IEEE, pages 1-5. IEEE, 2010.

[53] A Gomes, $\mathrm{CH}$ Antunes, and AG Martins. A multiple objective approach to direct load control using an interactive evolutionary algorithm. Power Systems, IEEE Transactions on, 22(3):1004-1011, 2007.

[54] A. Belov, V. Kartak, A. Vasenev, N. Meratnia, and Paul J.M. Havinga. Hierarchical scheme for balancing user comfort and electricity consumption of tank water heaters. In ISGT NA 2016. IEEE, 2016.

[55] Vassilis Kapsalis, Georgia Safouri, and Loukas Hadellis. Cost/comfort-oriented optimization algorithm for operation scheduling of electric water heaters under dynamic pricing. Fournal of cleaner production, 198:1053-1065, 2018.

[56] Vassilis Kapsalis and Loukas Hadellis. Optimal operation scheduling of electric water heaters under dynamic pricing. Sustainable Cities and Society, 31:109-121, 2017.

[57] Frederik Ruelens, Bert J Claessens, Salman Quaiyum, Bart De Schutter, R Babuska, and Ronnie Belmans. Reinforcement learning applied to an electric water heater: From theory to practice. IEEE Transactions on Smart Grid, 9(4):3792-3800, 2016.

[58] Bo Chai, Zaiyue Yang, and Jiming Chen. Optimal residential load scheduling in smart grid: A comprehensive approach. In 2013 9th Asian Control Conference (ASCC), pages 1-6. IEEE, 2013.

[59] Chengshan Wang, Yue Zhou, Jidong Wang, and Peiyuan Peng. A novel traversaland-pruning algorithm for household load scheduling. Applied energy, 102:1430-1438, 2013.

[60] Pengwei Du and Ning Lu. Appliance commitment for household load scheduling. Smart Grid, IEEE Transactions on, 2(2):411-419, 2011.

[61] Tanuja Bapat, Neha Sengupta, Sunil Kumar Ghai, Vijay Arya, Yedendra Babu Shrinivasan, and Deva Seetharam. User-sensitive scheduling of home appliances. In Proceedings of the 2nd ACM SIGCOMM workshop on Green networking, pages 43-48. ACM, 2011.

[62] Michael Angelo Pedrasa, ED Spooner, and IF MacGill. Improved energy services provision through the intelligent control of distributed energy resources. In PowerTech, 2009 IEEE Bucharest, pages 1-8. IEEE, 2009. 
[63] Michael Angelo A Pedrasa, Ted D Spooner, and Iain F MacGill. Coordinated scheduling of residential distributed energy resources to optimize smart home energy services. IEEE Transactions on Smart Grid, 1(2):134-143, 2010.

[64] Supun Tiptipakorn and Wei-Jen Lee. A residential consumer-centered load control strategy in real-time electricity pricing environment. In Power Symposium, 2007. NAPS'07. 39th North American, pages 505-510. IEEE, 2007.

[65] Jiangfeng Zhang and Xiaohua Xia. Best switching time of hot water cylinder-switched optimal control approach. In AFRICON 2007, pages 1-7. IEEE, 2007.

[66] Ning Lu and Srinivas Katipamula. Control strategies of thermostatically controlled appliances in a competitive electricity market. In Power Engineering Society General Meeting, 2005. IEEE, pages 202-207. IEEE, 2005.

[67] B Rautenbach and IE Lane. The multi-objective controller: a novel approach to domestic hot water load control. Power Systems, IEEE Transactions on, 11(4):1832-1837, 1996.

[68] Chong Hock K Goh and Jay Apt. Consumer strategies for controlling electric water heaters under dynamic pricing. In Carnegie Mellon Electricity Industry Center Working Paper, 2004.

[69] Alliander N.V. Consolidated annual results 2016 of alliander n.v. Technical report, 2016.

[70] George Koutitas. Control of flexible smart devices in the smart grid. Smart Grid, IEEE Transactions on, 3(3):1333-1343, 2012.

[71] Refrigerationg American Society of Heating and Air-Conditioning Engineers (ASHRAE). Thermal environmental conditions for human occupancy. Ashrae, 2010.

[72] EUROPEAN COMMITTEE FOR STANDARDIZATION. Ergonomics of the thermal environment - analytical determination and interpretation of thermal comfort using calculation of the pmv and ppd indices and local thermal comfort criteria. Iso, 2005.

[73] Refrigerationg American Society of Heating and Air-Conditioning Engineers (ASHRAE). ASHRAE Handbook-Fundamentals. ASHRAE Editor, Atlanta, GA, USA, i-p edition, 2013.

[74] Ken Parson Parson, Ken Parson, Ken Parson, Ken Parson, and Ken Ken Parson. Human thermal environments: The effects of hot, moderate, and cold environments on human health, comfort, and performance. CRC press, 2014.

[75] Valma J Robertson, Alex Ward, John Low, and Ann Reed. Electrotherapy explained: principles and practice. Elsevier Health Sciences, 2006.

[76] Zhijie Xu, Ruisheng Diao, Shuai Lu, Jianming Lian, and Yu Zhang. Modeling of electric water heaters for demand response: A baseline pde model. IEEE Transactions on Smart Grid, 5(5):2203-2210, 2014. 
[77] PJC Nel, Marthinus J Booysen, and Brink van der Merwe. A computationally inexpensive energy model for horizontal electric water heaters with scheduling. IEEE Transactions on Smart Grid, 9(1):48-56, 2016.

[78] Ruisheng Diao, Shuai Lu, Marcelo Elizondo, Ebony Mayhorn, Yu Zhang, and Nader Samaan. Electric water heater modeling and control strategies for demand response. In 2012 IEEE power and energy society general meeting, pages 1-8. IEEE, 2012.

[79] YM Han, RZ Wang, and YJ Dai. Thermal stratification within the water tank. Renewable and Sustainable Energy Reviews, 13(5):1014-1026, 2009.

[80] RP van Leeuwen. Towards 100role of smart control. $\mathrm{PhD}$ thesis, PhD Thesis, University of Twente, 2017.

[81] Eugenio García-Marí, María Gasque, Rosa Penélope Gutiérrez-Colomer, Federico Ibáñez, and Pablo González-Altozano. A new inlet device that enhances thermal stratification during charging in a hot water storage tank. Applied Thermal Engineering, 61(2):663-669, 2013.

[82] N Gopalakrishnan and S Srinivasa Murthy. Mixed convective flow and thermal stratification in hot water storage tanks during discharging mode. Applied Solar Energy, 45(4):254-261, 2009.

[83] MK Sharp and RI Loehrke. Stratified thermal storage in residential solar energy applications. Journal of Energy, 3(2):106-113, 1979.

[84] HCA Hoekstra, J Hamaker, CWJ van Koppen, and JTT van Wolde. The solar house of the eindhoven university of technology. In fournees internationales d'etudes sur le chauffage solaire dans le batiment, Liege, 12-14 Septembre 1977. AIM, 1977.

[85] US DOE. Energyplus engineering reference. The Reference to EnergyPlus Calculations, 2013.

[86] Inventum. Edr series. https://www. inventum.com/producten/ edr-80- laagvermogen/. Last visit:03/03/2020.

[87] A. Lekov D. Winiarski G. Rosenquist J. Lutz, C. Dunham. Wham: A simplified energy consumption equation for water heaters. In ACEEE Proceedings. ACEEE, 1998.

[88] Flink Energy Consulting. Electric water heaters as grid energy storage. Technical report, 2017.

[89] William M. Healy. The dependence of water heater energy factor on deviations from nominal conditions. Technical report, 2017.

[90] Torgeir Ericson. Direct load control of residential water heaters. Energy Policy, 37(9):3502-3512, 2009.

[91] United States Environmental Protection Agency. https : / /www . epa.gov/ watersense/bathroom-faucets. Last visit:05/07/2020. 
[92] Crosswater Digital. http : / /www . crosswater . co . uk. Last visit:05/07/2020.

[93] Stepon. http : //www. stepon.com.cn/chanpin/zhengqifang/. Last visit:05/07/2020.

[94] Charlie Huizenga, Zhang Hui, and Edward Arens. A model of human physiology and comfort for assessing complex thermal environments. Building and Environment, 36(6):691-699, 2001.

[95] Dusan Fiala. Dynamic simulation of human heat transfer and thermal comfort. $\mathrm{PhD}$ thesis, HOCHSCHULE FÜR TECHNIK, 1998.

[96] PO Fanger. Radiation and discomfort. ASHRAE journal, 28(2):33-34, 1986.

[97] Kenneth W Feldman. Help needed on hot water burns. Pediatrics, 71(1):145-146, 1983.

[98] A Re Moritz and FC Henriques Jr. Studies of thermal injury: Ii. the relative importance of time and surface temperature in the causation of cutaneous burns. The American journal of pathology, 23(5):695, 1947.

[99] Kenneth W Feldman, Robert T Schaller, Janen A Feldman, and Mollie McMillon. Tap water scald burns in children. Injury Prevention, 4(3):238-242, 1998.

[100] J Chatonnet and M Cabanac. The perception of thermal comfort. International Journal of Biometeorology, 9(2):183-193, 1965.

[101] Kuno Satoru. Comfort and pleasantness. In In Proceedings of Pan Pacific Symposium on Building and Urban Environmental Conditioning in Asia,, pages 283-392, 1995.

[102] Myron L Cohen. Measurement of the thermal properties of human skin. a review. Journal of investigative dermatology, 69(3):333-338, 1977.

[103] Alice M Stoll. Thermal properties of human skin related to nondestructuve measurement of epidermal thickness. Journal of Investigative Dermatology, 69(3):328-332, 1977.

[104] NZ Azer and S Hsu. The prediction of thermal sensation from a simple model of human physiological regulatory response. ASHRAE Trans, 83(Pt 1), 1977.

[105] University of Calgary. Somatosensory system. http : / / www . ucalgary . ca/ pip369/mod7/touch/somato2, 2016. Last visit:20/05/2017.

[106] Eric R Kandel, James H Schwartz, Thomas M Jessell, Steven A Siegelbaum, and A James Hudspeth. Principles of neural science, volume 4. McGraw-hill New York, 2000.

[107] HumanPhysiologyAcademy. Sensory receptors. http : //humanphysiology . academy/Neurosciences\%202015/BS\%20Tutorials/T. 2\% 20Sensory\%20Receptors . html, 2015. Last visit:20/05/2017.

[108] GT Coleman, H Bahramali, HQ Zhang, and MJ Rowe. Characterization of tactile afferent fibers in the hand of the marmoset monkey. Fournal of neurophysiology, 85(5):1793-1804, 2001. 
[109] InterNACHI. Inspecting water heater tanks in residential dwelling units. course document. Technical report, International Association of Certified Home Inspectors, 2015 .

[110] Co. Watts Water Technologies. Thermostatic mixing valves.

[111] William Reid. Thermostatic mixing value, 092004.

[112] LLC Plumbing Supply Group. Pressure balance vs. thermostatic shower valves.

[113] Grohe. https://www.grohe.nl/n1_nl/badkamer/ rainshower-smartcontrol-inbouw. html, 2020. Last visit:08/05/2020.

[114] Kohler. https://www. smarthome.kohler.com/ smart-showering, 2020. Last visit:08/05/2020.

[115] Quooker. https : / / www. quooker.co.uk, 2020. Last visit:08/05/2020.

[116] Richard Bellman. Dynamic programming. Dover, 2003.

[117] F. Gembicki and Y. Haimes. Approach to performance and sensitivity multiobjective optimization: The goal attainment method. IEEE Transactions on Automatic Control, 20(6):769-771, 1975.

[118] Joakim Widén, Magdalena Lundh, Iana Vassileva, Erik Dahlquist, Kajsa Ellegård, and Ewa Wäckelgård. Constructing load profiles for household electricity and hot water from time-use data-modelling approach and validation. Energy and Buildings, 41(7):753-768, 2009.

[119] Jo Kaye. Action a2a development of concept and offering renew : Development of concept and offering. Technical report, Energy Saving Trust Limited, Waterwise Project, United Kingdom, 2009.

[120] EngineeringToolbox. Average water usage per activity. https://Www . engineeringtoolbox.com/water-use-activity-d_1900.html. Last visit:05/07/2020.

[121] Meerp preparatory study on taps and showers. Technical background report, 2014.

[122] O. Wolf R. Kaps. Green public procurement for sanitary tapware. Technical background report, 2013.

[123] U.S. Environmental Protection Agency. A new reason for using a shower. https : //www. epa.gov/watersense/showerheads. Last visit:05/07/2020.

[124] N Pflugradt and W Schufft. Analysing low-voltage grids using a behaviour based load profile generator. In International Conference on Renewable Energies and Power Quality, 2013. 



\section{List of Publications}

1. A. Belov, N. Meratnia, B.J. van der Zwaag, and P.J.M. Havinga, "An efficient water flow control approach for water heaters in direct load control". In proceedings Journal of Engineering \& Applied Sciences, vol. 9, no. 11, pp. 2106-2120, 2014.

2. A. Belov, A. Vasenev, P.J.M. Havinga, N. Meratnia, and B.J. van der Zwaag, "Reducing user discomfort in direct load control of domestic water heaters". In proceedings of 2015 IEEE Innovative Smart Grid Technologies - Asia (ISGT ASIA 2015), 3-6 November 2015, Bangkok, Thailand. pp. 1-6.

3. A. Belov, N. Meratnia, and P.J.M. Havinga, "Enhancing user comfort models for demand response solutions for domestic water heating systems". In proceedings of the 5th International Conference on Smart Cities and Green ICT Systems - IEEE PES SmartGreens, 23-25 April 2016, Italy, Rome. pp. 201-212.

4. A. Belov, V. Kartak, A. Vasenev, N. Meratnia, and P.J.M. Havinga, "A hierarchical scheme for balancing user comfort and electricity consumption of tank water heaters". In proceedings of the 2016 IEEE Power \& Energy Society Innovative Smart Grid Technologies Conference (ISGT NA 2016), 6-9 September 2016, Minneapolis, USA.

5. A. Belov, V. Kartak, A. Vasenev, N. Meratnia, and P.J.M. Havinga, "Load shifting of domestic water heaters under double price tariffs: Bringing together money savings and comfort". In proceedings of 2016 IEEE PES Innovative Smart Grid Technologies Europe, ISGT Europe 2016, 9-12 October Ljubljana, Slovenia. pp. 1-6.

6. A. Belov, A. Vasenev, V. Kartak, N. Meratnia, and P.J.M Havinga, "Peak load reduction of multiple water heaters: Respecting consumer comfort and money savings". In proceedings of 2016 IEEE Online Conference on Green Communications (OnlineGreenComm), 14 Nov.-17 Dec. 2016, Piscataway, NJ, USA.

7. A. Belov, V. Kartak, N. Meratnia, and P.J.M. Havinga, "Balancing electricity consumption and user comfort in load management of electric tank water heaters". In proceedings of the 21st International Workshop on Computer Science and Information Technologies (CSIT 2019), 2-4 October 2020, Vienna, Austria. pp. 38-44. 